\title{
Virtual Environment Applications for Buried Waste Characterization Technology Evaluation Report
}

Virtual Environment

Project Team

Published May 1995

Idaho National Engineering Laboratory Lockheed Idaho Technologies: Company Idaho Falls, Idaho 834.15

Prepared for the

U.S. Department of Energy

Assistant Secretary for Environmental Management

Under DOE Idaho Operations Office

Contract DE-AC07-94ID132:3 


\section{Virtual Environmental Applications for Buried Waste Characterization Technology Evaluation Report}

INEL-94/0080

Prepared by

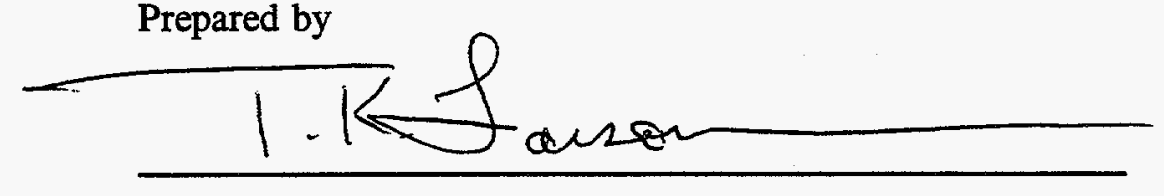

T. K. Larson, Principal Investigator,

Virtual Environment Project
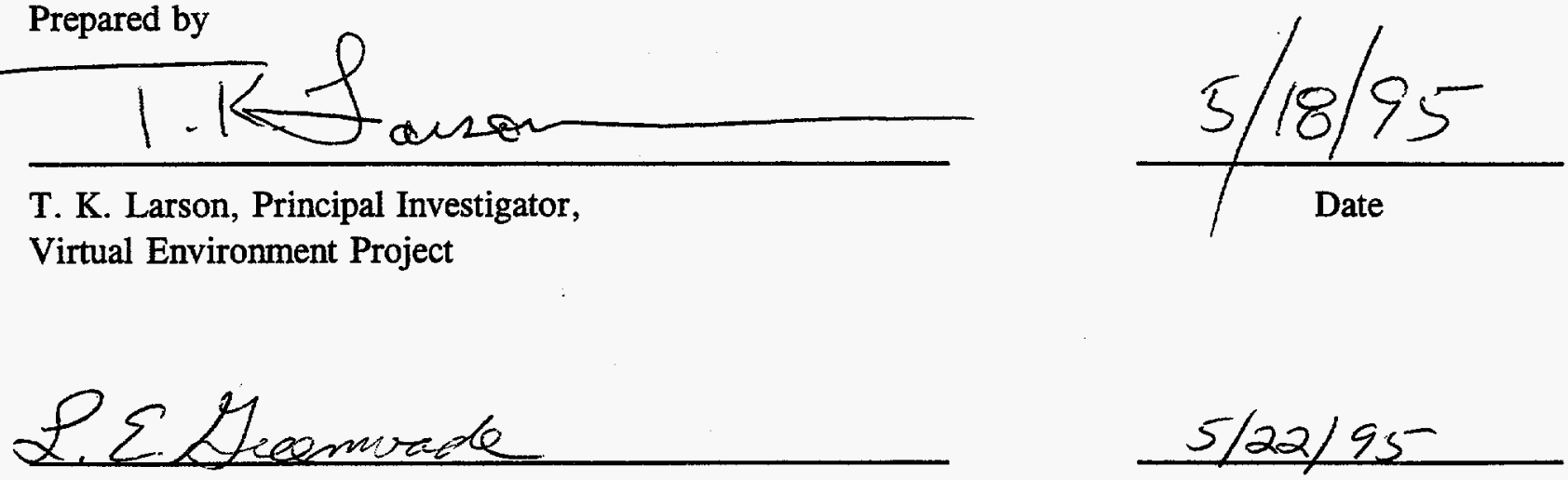

L. E. Greenwade, Principal Investigator,

Virtual Environment Project

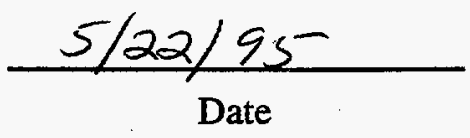

Reviewed by

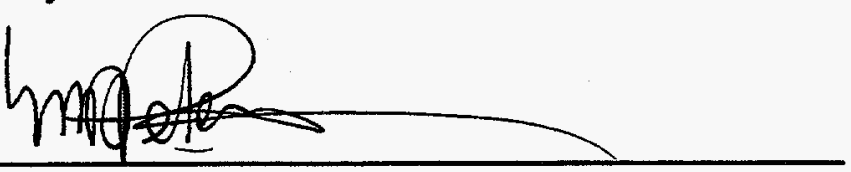

M. D. Peters, Project Manager,

Virtual Environment Project

$5 / 22 / 93$

Date

Approved by

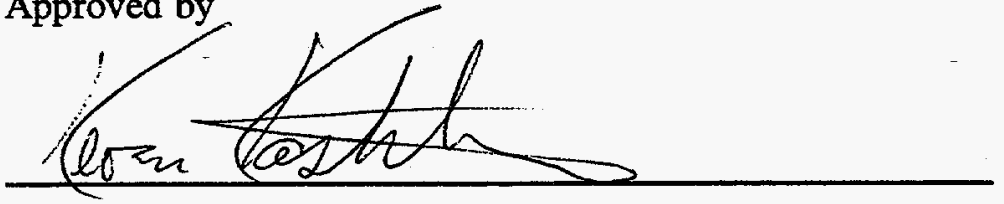

K. M. Kostelnik, Manager,

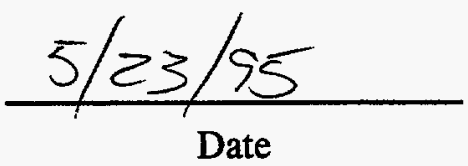

Environmental Restoration Technologies 


\section{DISCLAIMIER}

This report was prepared as an account of work sponsored by an agency of the United States Government. Neither the United States Government nor any agency thereof, nor any of their employees, make any warranty, express or implied, or assumes any legal liability or responsibility for the accuracy, completeness, or usefulness of any information, apparatus, product, or process disc:losed, or represents that its use would not infringe privately owned rights. Reference herein to any specific commercial product, process, or service by trade name, trademark, manufacturer, or otherwise does not necessarily constitute or imply its endorsement, recommendation, or favoring by the United States Government or any agency thereof. The views and opinions of authors expressed herein do not necessarily state or reflect those of the United States Government or any agency thereof. 


\section{DISCLAIMER}

Portions of this document may be illegible in electronic image products. Images are produced from the best available original document. 


\section{ABSTRACT}

The project, Virtual Environment Applications for Buried Waste Characterization, was initiated in the Buried Waste Integrated Demonstration Program in fiscal year 1994. This project is a research and development effort that supports the remediation of buried waste by identifying and examining the issues, needs, and feasibility of creating virtual environments using available characterization and other data. This document describes the progress and results from this project during the past year. 



\section{SUMMARY}

Virtual environments (VEs) use computers and display mechanisms to help users comprehend complex situations. The VE technology described in this report supports the remediation of buried waste by improving the access to and usefulness of site characterization information provided to the remedial decisionmakers or operators. This characterization information may come from historical records or from field data collected before remedial work or at the digface during the retrieval operation. By improving the usefulness of information, the VEs and advanced visualization techniques support risk evaluation, before beginning operations and during the remedial operations.

The objective of this work was to investigate the feasibility and usefulness of simulation-based planning and design concepts for restoring and cleaning up subsurface hazardous waste sites. In particular, the feasibility of using available characterization data from waste sites at the Idaho National Engineering Laboratory (INEL) to generate a VE (computer-generated virtual world) representing the site and the usefulness of that environment in remediation assessment and planning activities were examined.

To define the feasibility and opportunities for the using VE techniques, the following specific objectives were defined:

1. Develop a rapid prototype for soliciting user ideas

2. Define functional requirements for a prototype system

3. Develop a hardware and software prototype platiorm based on defined functional requirements.

Based on the early evaluation of the rapid prototype system, additional objectives for development in 1994 were defined:

4. Develop depth-to-object interpretation algorithms and automate these for use in digface data interpretation

5. Investigate the applicability of an automatic target recognition system (previously developed for a separate use) for interpreting data from digface sensors to define waste types or objects

6. Investigate the applicability of neural networks for interpreting data from digface sensors to define waste types or objects

7. Investigate the applicability of a visual data base for presenting historical records and possible integrating the records with sensor data to improve understanding of available information on waste sites

8. Investigate the use of immersion as a tool used by operators or decisionmakers to improve understanding of characterization data

9. Investigate potential product commercialization and faciors affecting marketability of project results. 
Advances, conclusions, and recommendations for each objective are provided in this report.

\section{Rapid Prototype Development}

The initial work scope for this project involved generating rapid prototype environments. These rapid prototypes provided baseline environments that could be shown to potential users to assist them in understanding current capabilities and to solicit input about needed and desired functionality. The Characterization Cell in the Cold Test Pit (CTP) at the INEL was used for this work because the engineered facility is well defined and significant engineering and geophysics survey characterization data are readily available.

Three different rapid prototype environments were constructed, including a walkthrough of the Characterization Cell, a visual data base of the Characterization Cell, and an example three-dimensional data display. These environments were shown to and discussed with potential end users including geophysicists, engineers, and Environmental Restoration representatives. As a result of the initial evaluations, functional requirements for a prototype integrated hardware and software platform were generated.

\section{Integrated Hardware and Software Platform}

A system that integrates different functionalities into a single overall platform was designed and implemented in prototype form. The integrated system has access to data, data base information, and analysis tools that may be of interest to or used for buried waste characterization applications.

The integrated hardware design and software platform was based on functional descriptions of needed analysis, archival, and user interaction capabilities. These requirements were based on the operator being able to interactively manipulate any reasonable subset of the available data in real time. The data under investigation must be easily and readily accessible for analysis, manipulation, and display in any manner desired by the user. In addition, the methods and results of the analyses should be stored to provide a knowledge base for future activities.

The functional design consists of seven components, five are software and two are hardware. All of the components are specified so that off-the-shelf solutions can be used. As faster, more flexible or otherwise superior components become available, they may be integrated into the system at minimal expense and rework. The simplest to implement and describe are the hardware components consisting of a Rendering Environment and the User Interaction Environment. The software units are designed to accomplish the tasks identified in the requirements analysis, including data analysis, representing and manipulating the virtual world, semiautonomous data analysis, access to external simulation capabilities, and a high performance, distributed scientific data base.

Software packages used in the integrated system are Application Visualization System, for the display environment; WorldToolKit (the current standard) for the virtual world authoring environment, and Aurora Data Server from Xidak for the data archiving subsystem.

The primary hardware components for the core system are a Sun Microsystems SparcServer 2000 with eight $50 \mathrm{Mhz}$ processors, each with $2 \mathrm{MB}$ of external cache. 
Currently, the major systems components are loosely coupled and improvement of the system is ongoing. Major improvement will take place after a formal review of the prototype capabilities by potential users, specifically Environmental Restoration personnel. Additional development should be guided by reviewer comments.

\section{Depth-to-Object Algorithms}

During the rapid prototype review, geophysicists stressed the need for an analysis system that could support extraction of relationships in the geophysics survey data. The following analysis areas are major areas of interest:

- Depth-to-object estimation

- Contour analysis (determination of anomaly extrema)

- Anomaly characterization.

Software that performs depth-to-object analyses has been implemented and verified. The algorithms provide reasonable estimates of the object depth, although the range of estimates is wide. Depths estimated using the digface and Rapid Geophysical Surveyor data were typically within one-half foot of real values. Automation of the depth estimation algorithms allows verification to be done on numerous data. Such verification is too tedious and time consuming to be done by hand. Based on encouraging results from the automated algorithms, numerous data sets, comprising a range of targets and environments should be verified. The 1994 digface data set is one specific data set on which the algorithms should be checked.

\section{Automatic Target Recognition System Evaluation}

An Automatic Target Recognition (ATR) system was evaluated for data interpretation of the sensor data obtained during the digface characterization process. The potential role of the ATR system is to aid the operator or geophysicist. The ATR system could be used to automatically scan incoming data and highlight potential target areas. Targets would be defined by expert users during the training process. The potential targets would then be verified by a human expert. The system would allow the expert to focus attention on the areas of suspected targets when making characterization determinations.

The ATR system performance was generally good for data interpretation. (For this initial study, the evaluation was done under rather ideal conditions.) Although there were errors in distinguishing boxes from barrels, the system is capable (based on the limited testing done) of distinguishing target objects from background soil.

Initial results indicate that further evaluation and development of the ATR system are needed. Initial results are encouraging; however, the ATR system must be demonstrated under conditions that represent actual buried waste sites. 


\section{Neural Network Evaluation}

Artificial neural networks can potentially scan large amounts of data rapidly to highlight regions of interest similar to the ATR system. The two methods use different approaches, each with advantages and disadvantages. Neural networks were evaluated for potential data interpretation application.

The neural network performance was generally good for digface data interpretation. Both backpropagation and radial basis function neural network paradigms were used for target background classification. After training, the networks demonstrated reasonable capability to distinguish targets from background. The networks have been distinguishing target and background locations accurately on over $85 \%$ of the digface data.

Networks were trained using various combinations of training input, including single sensor types from a single data level, single sensor types from multiple levels of data, multiple sensor types from a single data level, and multiple sensor types from multiple data levels. Using multiple levels of data and combining sensor types for training and testing resulted in better definition of target boundaries.

A training set that consisted of data from several experiments was generated to train a network that would produce good results on all digface experiments. Targets from each digface experiment (except Experiments 1 and 4) were included in this training set. Reasonable results were achieved.

Although evaluation is still in progress, preliminary indications suggest that neural networks can provide reasonable object depth estimates for the digface data collected in fiscal year 1993. Predictions to date are within about $10 \%$ of the documented object depths.

Initial results indicate that further evaluation and development of neural networks are needed. Initial results are encouraging; however, the networks must be demonstrated under conditions representing actual buried waste sites.

\section{Visual Data Base Evaluation}

The integrated hardware and software platform was applied to two visual data base problems. These were real world implementations of the functional design requirements and provided an opportunity to test the usability of the system in real scenarios. Several individuals expressed interest in being able to visually inspect, manipulate, and query large data bases containing historical records of waste disposal areas. The two applications, CTP Characterization Cell and Pit 9, were chosen because of data availability and relevance to the program.

The visual data base applications showed the ability, benefit, and utility of having different sources of information about a particular waste site gathered into a centralized location. For operational usefulness, the need to have computing capabilities with significant power was demonstrated. The visual data base implementation was run on several different classes of work stations. On newer machines (e.g., with dedicated graphics processors), the performance was much better and movement through the objects in the world was acceptable. 
Based on implementation experience with the Pit 9 data base, it is clear that there will always be some doubts about the accuracy of the information in such a historical data base. The information must be used cautiously and with human expertise, and experience must be used in the process of building and using a visual data base.

The current system should be formally reviewed by potential users, specifically Environmental Restoration personnel. Subsequent development, if needed, should be guided by reviewer comments.

\section{Evaluation of Immersion}

An evaluation of potential benefits of the system, including inmersive capabilities, has been done. One advantage of an immersive system is the ability to use the high bandwidth communication system that comprises the human optical system. There are many degrees of immersion varying from the minimal capabilities of a video monitor to the total immersion provided by stereo head-mounted displays. For each application, the appropriate degree of immersion needed for efficiently completing designated tasks should be considered.

Initial efforts focused on how immersion might benefit interpreting of geophysical data. Current near-field geophysics data interpretation requires substantial training and expertise and is in an immature state. Until techniques are developed to support data representation by physical world objects, it is not clear how immersion will benefit geophysical data interpretation. Irnmersion will play a larger role in the planned use of stereo/video and stereo/video mixing with VEs. Immersion will improve depth perception of people operating telerobotic equipment in hazardous environments. Further evaluation is planned in 1995.

\section{Commercialization}

Preliminary work has been done to evaluate the potential market for the VE technologies under development. The market assessment also included initial evaluation of factors to be considered in system development from the point of view of ultimate product usefulnes: and commercialization potential.

The potential market for buried waste remediation technology is large and extends beyond Federal installations such as the U.S. Department of Energy and the U.S. Department of Defense sites. The Federal sites represent a significant potential investment of resources for cleanup; the U.S. Environmental Protection Agency estimates range in the hundreds of billions of dollars.

A preliminary market survey supports the project's assumption VE technologies usefulness, based on feedback from businesses involved in the remediation. The conmercial support leans toward a suite of technologies including VE and assumes a fairly sophisticated site characterization capability as part of the VE system. Potential markets for spin-off products from the buried waste VE work have been identified. These include the public utilities industry (e.g., underground gas and water lines), land-use planning and construction industries (e.g., imaging and displaying subterranean infrastructure), and industrial archeology (e.g., detection of underground structures). Similar to the buried waste characterization problem, all of these sidemarkets are potential users of VE technologies, assuming that the techniques are integrated with a sophisticated sensing and detertion capability. The market support 
found in the initial survey is largely predicated on further developing both the sensor and VE display capabilities.

The lack of standardization of virtual worlds software is a general problem and will affect commercial potential of project products. However, it is anticipated that by 1996 , the market for virtual worlds software will change remarkably. Constant scrutiny of this area and adjustment of project tools and software are advised. 


\section{ACKNOWLEDGMENTS}

With the efforts of the Virtual Environment Project team, the Virtual Environment Applications for Buried Waste Characterization Technology Evaluation Report was successfully completed. The authors include Jim Anderson, Dick Callow, Chris Cullen, Eric Greenwade, Dave Harker, Pam Johnson, Tom Larson, and Dr. Gordon Lassahn from the Idaho National Engineering Laboratory, Dr. Mary Poulton and Marshall Brown from the University of Arizona, and Dr. Robert Jacobson from Worldesign, Inc. 


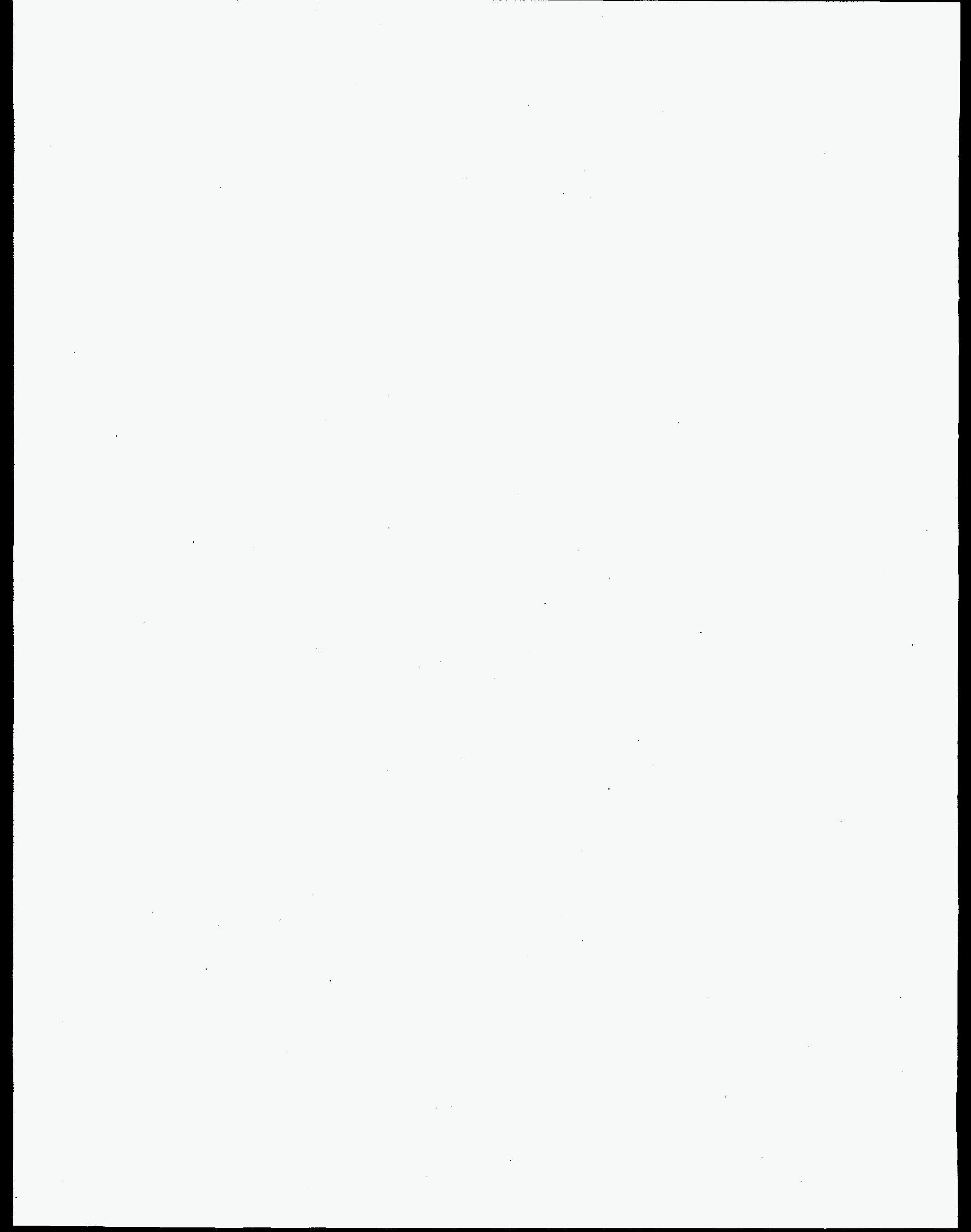




\section{CONTENTS}

ABSTRACT $\ldots \ldots \ldots \ldots \ldots \ldots \ldots \ldots \ldots \ldots \ldots \ldots \ldots \ldots$ iii

SUMMARY $\ldots \ldots \ldots \ldots \ldots \ldots \ldots \ldots \ldots \ldots \ldots \ldots \ldots \ldots \ldots$

ACKNOWLEDGMENTS $\ldots \ldots \ldots \ldots \ldots \ldots \ldots \ldots \ldots \ldots \ldots \ldots \ldots \ldots$

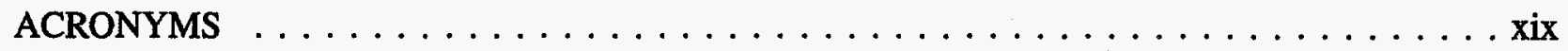

1. TECHNOLOGY DESCRIPTION $\ldots \ldots \ldots \ldots \ldots \ldots \ldots \ldots \ldots \ldots$

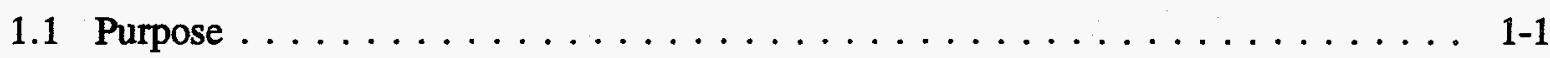

1.2 Virtual Environments and Virtual Reality Technology Overview . . . . . . . . 1-1

1.3 Technology Need $\ldots \ldots \ldots \ldots \ldots \ldots \ldots \ldots \ldots \ldots \ldots \ldots \ldots$

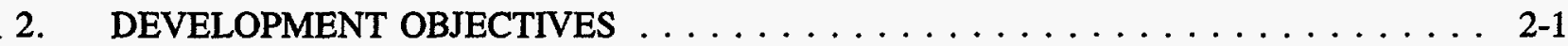

3. QUALITY PROCEDURES, HEALTH AND SAFETY, ENVIRONMENTAL

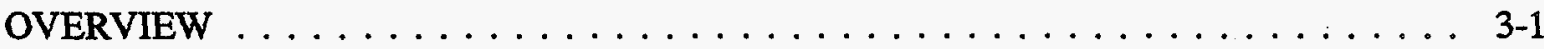

3.1 Quality Procedures $\ldots \ldots \ldots \ldots \ldots \ldots \ldots \ldots \ldots \ldots \ldots \ldots \ldots \ldots$

4. PROJECT DESIGN AND DEVELOPMENT PHILOSOPHY $\ldots \ldots \ldots \ldots \ldots$. . . . 4-1

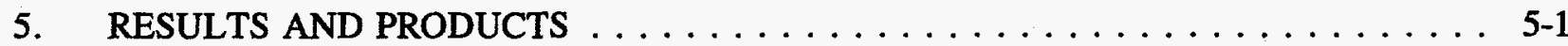

5.1 Rapid Prototype Environments $\ldots \ldots \ldots \ldots \ldots \ldots \ldots \ldots \ldots \ldots \ldots$

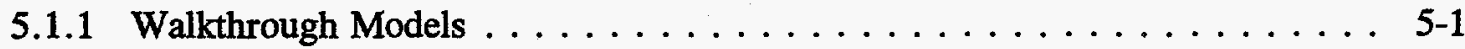

5.1 .2 Visual Data Base Model . . . . . . . . . . . . . . . . . 5-3

5.1.3 Three-Dimensional Data Display/Analysis Environment . . . . . . . . . 5-5

5.1 .4 User Feedback On Rapid Prototype . . . . . . . . . . . . . . . . . . . . 5-9

5.2 Functional Requirements Definition $\ldots \ldots \ldots \ldots \ldots \ldots \ldots \ldots \ldots$

5.3 Development of Integrated Software $\ldots \ldots \ldots \ldots \ldots \ldots \ldots \ldots$

5.4 Depth-to-Object Algorithms $\ldots \ldots \ldots \ldots \ldots \ldots \ldots \ldots \ldots \ldots$

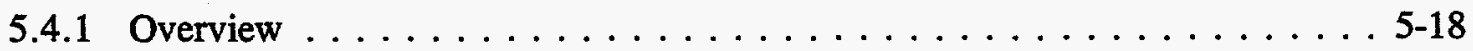

5.4 .2 Digface Data Analysis Software . . . . . . . . . . . . . . . . 5-19

5.5 Automatic Target Recognition Evaluation $\ldots \ldots \ldots \ldots \ldots \ldots \ldots \ldots$ 
5.5 .1 Automatic Target Recognition System Overview . . . . . . . . . . . 5-41

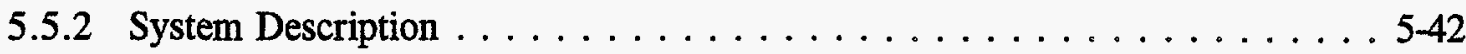

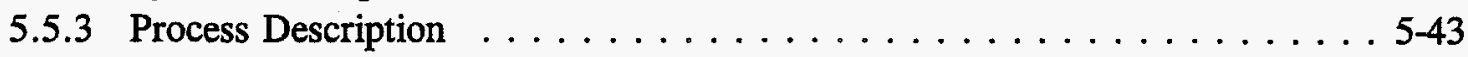

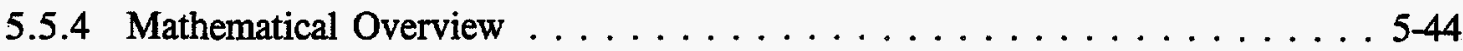

5.5 .5 Application To Digface Data Interpretation . . . . . . . . . . 5-45

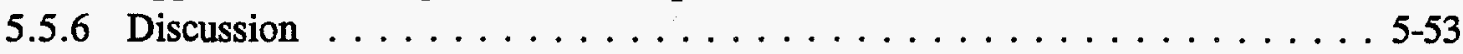

5.5 .7 Conclusions and Recommendations . . . . . . . . . . . 5-64

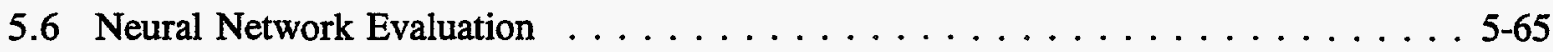

5.6 .1 Background on Neural Networks $\ldots \ldots \ldots \ldots \ldots \ldots \ldots \ldots$

5.6 .2 Classification Task Paradigms . . . . . . . . . . . . . . . 5-67

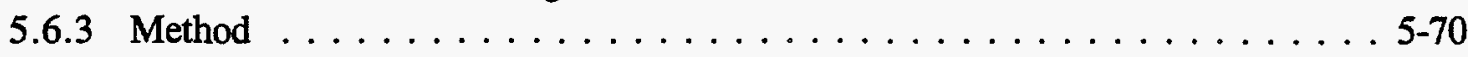

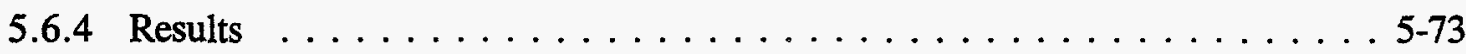

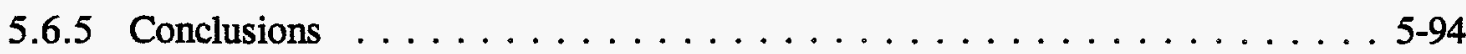

5.7 Application of Visual Data Base $\ldots \ldots \ldots \ldots \ldots \ldots \ldots \ldots \ldots$

5.7.1 Cold Test Pit Descriptive Data Base . . . . . . . . . . . . . . . . . . 5 599

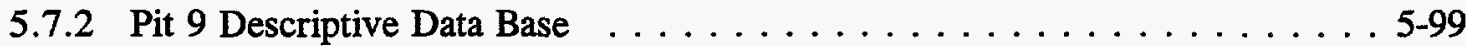

5.7 .3 Visual Data Base Implementation . . . . . . . . . . . . . . . . 5-101

5.7 .4 Generating WTK Objects for Pit $9 \ldots \ldots \ldots \ldots \ldots \ldots$. . . . . . . . . . . . . . . . . . . . . . . . . . . .

5.7 .5 Current Status and Future Directions . . . . . . . . . . . . . 5-113

5.7.6 Conclusions and Recommendations . . . . . . . . . . . . . . . 5-119

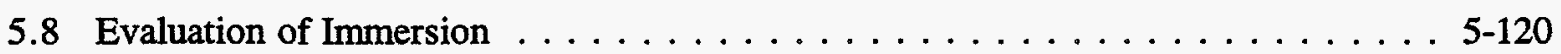

6. INSIGHTS, ISSUES, PROBLEMS $\ldots \ldots \ldots \ldots \ldots \ldots \ldots \ldots \ldots$

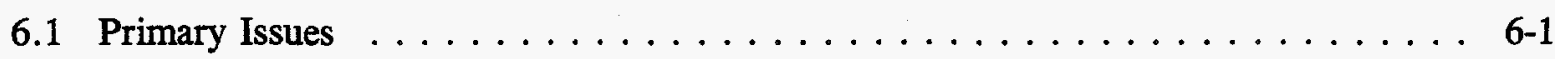

6.1 .1 Unstructured Nature of the Digface . . . . . . . . . . . . . . 6 6-1

6.1.2 Role of Human in the Remediation Process . . . . . . . . . . . . . . . 6-1

6.1 .3 Overburden Removal and Waste Seam Retrieval . . . . . . . . . . . . 6-2

6.1 .4 Learning from Experience $\ldots \ldots \ldots \ldots \ldots \ldots \ldots \ldots \ldots \ldots$

6.1 .5 Operator Qualification Requirements ................ 6-5

6.2 Secondary Issues $\ldots \ldots \ldots \ldots \ldots \ldots \ldots \ldots \ldots \ldots \ldots$

6.2.1 Stereovision and Virtual Environments (Mixing) . . . . . . . . . . 6-6

6.2 .2 Forward Modeling of Sensors . . . . . . . . . . . . . 6 6-6

7. FULL-SCALE IMPLEMENTATION, RELATED EFFORTS, AND COMMERCIALIZATION $\ldots \ldots \ldots \ldots \ldots \ldots \ldots \ldots \ldots \ldots \ldots \ldots$

7.1 Full-Scale Implementation $\ldots \ldots \ldots \ldots \ldots \ldots \ldots \ldots \ldots \ldots \ldots \ldots \ldots$ 


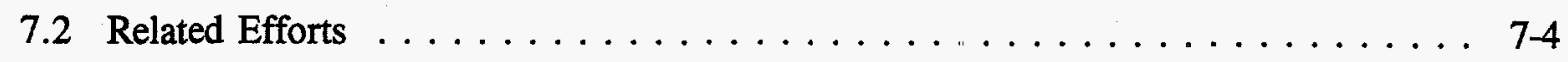

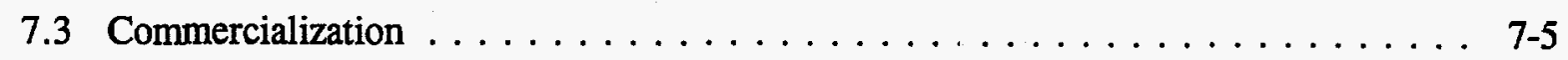

8. CONCLUSIONS, RECOMMENDATIONS, DEVELOPMENT NEEDS $\ldots \ldots \ldots \ldots$ 8-1

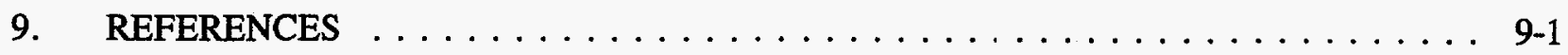

Appendix A-FY-93 Digface Performance Tests $\ldots \ldots \ldots \ldots \ldots \ldots \ldots \ldots$ A-1

Appendix B-Characterization Cell VREAM Model Screen Images $\ldots \ldots \ldots \ldots \ldots \ldots$ B-1

Appendix $\mathbf{C}-$ Neural Network Supplemental Information $\ldots \ldots \ldots \ldots \ldots \ldots$ C-1

Appendix D-Descriptions of the RWMIS and Qualifier Data Base Fields $\ldots \ldots \ldots \ldots$ D-1

Appendix E-Printouts of RWMIS and Qualifier Data Bases $\ldots \ldots \ldots \ldots \ldots \ldots$ E-1

Appendix F-Additions to WorldToolKit Source Code $\ldots \ldots \ldots \ldots \ldots \ldots \ldots$ F-1

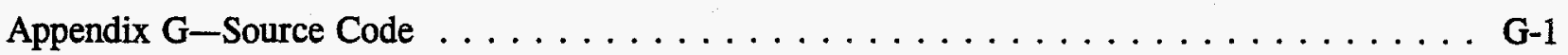

\section{FIGURES}

4-1. Overall design schematic of VE system $\ldots \ldots \ldots \ldots \ldots \ldots \ldots \ldots \ldots \ldots$ 4-1

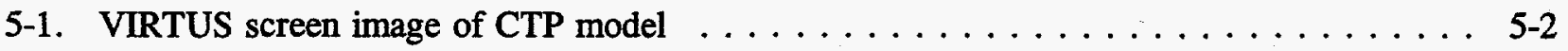

5-2. Screen image of VREAM model of CTP $\ldots \ldots \ldots \ldots \ldots \ldots \ldots \ldots \ldots . \ldots \ldots$

5-3. Advanced Visual Systems-generated three-dimensional image $\ldots \ldots \ldots \ldots \ldots \ldots$ 5-7

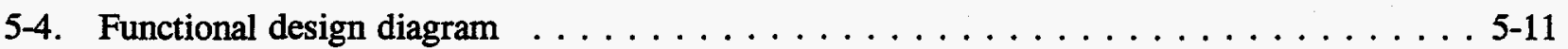

5-5. Schematic of how analysis interface might appear $\ldots \ldots \ldots \ldots \ldots \ldots \ldots \ldots$ 5-15

5-6. Flow chart for the GRIDIT program $\ldots \ldots \ldots \ldots \ldots \ldots \ldots \ldots \ldots \ldots$ 5-21

5-7. Flow chart for the SEARCH $\operatorname{program} \ldots \ldots \ldots \ldots \ldots \ldots \ldots \ldots \ldots \ldots \ldots \ldots \ldots$

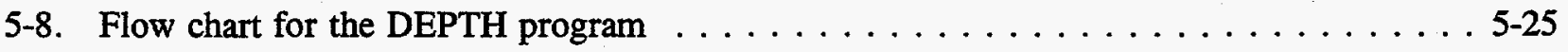

5-9. $+\mathrm{X}$ depth estimation results for RGS peak at $\mathrm{X}=89$ and $\mathrm{Y}=227 \mathrm{ft} \ldots \ldots \ldots \ldots$ 5-28

5-10. $-\mathrm{X}$ depth estimation results for RGS peak at $\mathrm{X}=89$ and $\mathrm{Y}=227 \mathrm{ft} \ldots \ldots \ldots \ldots$ 5-28

5-11. $+\mathrm{Y}$ depth estimation results for RGS peak at $\mathrm{X}=89$ and $\mathrm{Y}=227 \mathrm{ft} \ldots \ldots \ldots \ldots .5-29$ 
5-12. - $Y$ depth estimation results for RGS peak at $X=89$ and $Y=227 \mathrm{ft}$

5-13. Peak-to-valley depth estimation results for $R G S$ peak at $X=89$ and $Y=227 \mathrm{ft}$, valley

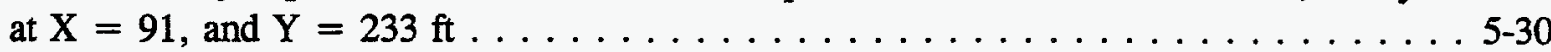

5-14. Buried objects and masks used in training $\ldots \ldots \ldots \ldots \ldots \ldots \ldots \ldots$

5-15. Application of system trained on three scenes to target detection in 30 testing scenes . . . 5-51

5-16. Search for barrels, linear combination, four training scenes $\ldots \ldots \ldots \ldots \ldots \ldots \ldots$

5-17. Search for barrels, quadratic combination $\ldots \ldots \ldots \ldots \ldots \ldots \ldots \ldots \ldots \ldots \ldots \ldots$

5-18. Search for boxes, quadratic, two training scenes $\ldots \ldots \ldots \ldots \ldots \ldots \ldots \ldots$

5-19. Search for boxes, quadratic, one training scene $\ldots \ldots \ldots \ldots \ldots \ldots \ldots$

5-20. Search for barrels, peak width, first training set. $\ldots \ldots \ldots \ldots \ldots \ldots \ldots$

5-21. Search for barrels, peak width, second training set $\ldots \ldots \ldots \ldots \ldots \ldots$

5-22. A feed-forward, three layer neural network for supervised learning. . . . . . . . . 5-67

5-23. Gridded output for network trained with magnetic data from Experiment 2, level $2 \ldots \ldots$ 5-74

5-24. Gridded output for network trained with magnetic data from Experiment 2, level $5 \ldots$. . 5-75

5-25. Gridded output for network trained with EM data from Experiment 2, level $2 \ldots \ldots$. . . 5-77

5-26. Gridded output for network trained with EM data from Experiment 2, level $5 \ldots \ldots$. . . 5-78

5-27. Gridded output for network trained with magnetic and EM data from Experiment 2,

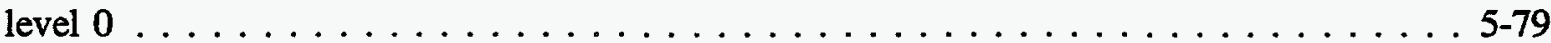

5-28. Gridded output for network trained with magnetic and EM data from Experiment 2,

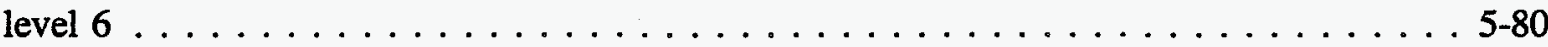

5-29. Gridded output from network trained with magnetic data from all levels of

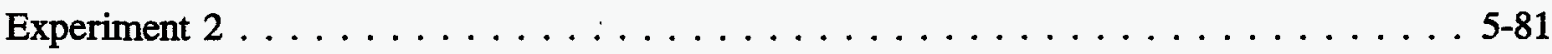

5-30. Gridded output from network trained with EM data from Experiment $2 \ldots \ldots$. . . . 52

5-31. Gridded output from network trained with magnetic and EM data from all levels of

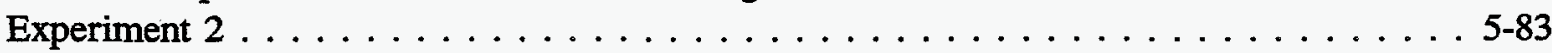

5-32. Sample shipping manifest for waste disposed of at Pit $9 \ldots \ldots \ldots \ldots$. $\ldots \ldots$. $\ldots \ldots$. 
5-33. Characterization Cell objects in the WTK environment

5-34. Characterization Cell objects in the WTK environment illustrating point and click option

5-35. Pit 9 monument locations

5-36. Pit 9 boundary locations and waste locations as reported in the shipping manifests 5-114

5-37. Illustration of the WTK Pit 9 world viewed from outside 5-115

5-38. Illustration of the WTK Pit 9 world viewed from inside

6-1. Schematic illustration showing function of digface characterization during overburden removal

6-2. Schematic illustration showing function of digface characterization during waste seam

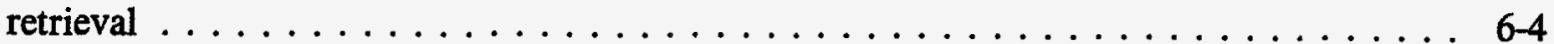

\section{TABLES}

5-1. Peak-to-valley locations determined by program SEARCH for northern RGS data

5-2. Results from depth estimation for RGS peak at $X=89 \mathrm{ft}$ and $\mathrm{Y}=227 \mathrm{ft}$

5-3. Depth and uncertainty estimates for object 7, Experiment 2

5-4. Power law depth analysis for object 6

5-5. Power law depth analysis for object 7 $5-36$

5-6. Power law depth analysis for object 11

5-7. Power law depth analysis for object 17

5-8. Digface characterization sensor description $5-49$

5-9. Automatic target recognition mask object relationship $5-50$

5-10. Digface characterization sensor description $5-72$

5-11. Summary of neural network training and testing results $5-84$

5-12. Interexperiment testing with neural network trained with Experiment 2 data $5-86$

5-13. Interexperiment testing with neural network trained with Experiment 3 data $5-87$ 
5-14. Interexperiment testing with neural network trained with Experiment 5 data

5-15. Interexperiment testing of neural network trained with Experiment 6 data

5-16. Summary of testing for importance of survey components in neural network training and testing

5-17. Summary of testing for importance of survey components in neural network training and testing

5-18. Summary of neural network predicted object depths

5-19. Summary of neural network conductivity estimates

5-20. Master data base entry for manifest in Figure 5-32

5-21. Container data base entry ( 1 of 2 ) for manifest in Figure 5-32

5-22. Nuclide data base entry for manifest in Figure 5-32 .

5-23. Qualifier flag data base entry for manifest in Figure 5-32

5-24. Data retrieved from Characterization Cell data base

5-25. Pit 9 monument locations

5-26. Example of parsing a location string 5-114 


\section{ACRONYMS}

ATR

AVS

BWID

CAVE

CIDRA

CR

CTP

DOD

DOE

EM

EPA

FY

GPR

HMD

ICERVS

INEL

LOD

PE

PVM

$\mathrm{P} / \mathrm{V}$

RBF

RDMS

RE
Automatic Target Recognition

Advanced Visual Systems

Buried Waste Integrated Demonstration

Computer Audiovisual Environment

Contaminant Inventory Data Base for Risk Assessment

Classification rate

Cold Test Pit

U.S. Department of Defense

U.S. Department of Energy

Electromagnetic

U.S. Environmental Protection Agency

Fiscal year

Ground Penetrating Radar

Head-mounted display

Interactive Computer Enhanced Remote Viewing System

Idaho National Engineering Laboratory

Levels of detail

Processing element

Portable Virtual Machine

Peak-to-valley

Radial basis function

Relational Data Base Management System

Rendering Environment 
RWMC Radioactive Waste Management Complex

RWMIS Radioactive Waste Management Information System

SBPD Simulation-based planning and design

SDA Subsurface Disposal Area

UCD Unstructured cell data

VE

Virtual Environment

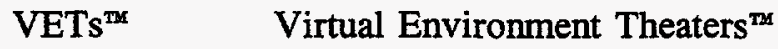

VWAE Virtual World Authoring Environment

WTK WorldToolKit 


\section{Virtual Environment Applications for Buried Waste Characterization Technology Evaluation Report}

\section{TECHNOLOGY DESCRIPTION}

\subsection{Purpose}

Virtual environments (VEs) use computers and display mechanisms to help users comprehend complex situations. The VE technology supports the remediation of buried waste by improving access to and the usefulness of site characterization information to remedial decisionmakers or operators. This characterization information may come from historical records or from field data collected before remedial work or at the digface during the retrieval operation. By improving the usefulness of information, the VEs and advanced visualization techniques support risk evaluation, before beginning operations, or more particularly, during the remedial operations.

The goal of the work described in this report was to examine the feasibility and usefulness of VE generation from available characterization information. Buried waste remediation brings with it complex situations of enormous breadth. Furthermore the science, technolojgy, and understanding associated with buried waste characterization is in an infant state. The work descrilsed here is not complete, and we have tried to cover many topics in addressing the goals of the project.

\subsection{Virtual Environments and Virtual Reality Technology Overview}

Advances in computing technology over the past decade make the concept of simulation-based planning and design (SBPD) feasible. The SBPD concept considers the use and integration of state-of-the-art simulation techniques to reduce the need for physical prototypes and physical simulation. SBPD will eventually establish a new paradigm that is an innovative method for fully utilizing available and developing tools to plan, design, analyze, develop, and produce affordable complex systems. Technologies considered crucial in SBPD include (a) intelligent, integrated, automated, real-time control of the design process, including proper sequencing of system models and data bases and design revision disciplines; (b) effective implementation of advanced visualization, including virtual reality, and multimedia techniques into the design process; (c) high-speed, wide bandwidth networking; (d) paralyzed processing and computing; and (e) virtual prototyping and physics-based modeling.

VEs, or computer-generated worlds, will play a significant role in this concept. Computer-generated worlds could take on different forms depending on the circumstances and the requirements of the situation. A graphics system works with a data base to describes objects, their position in coordinate space, and color and texture attributes. From this description, the graphics system renders a bit-map representation of the objects depending on the orientation of the observer and lighting sources. In some cases, the VE may be very realistic relative to the real environment, and the objects in the computer-generated world may appear nearly as they do in reality. In other cases, the VE may be a symbolic representation of the situation being simulated. For example, the representation of physical data may include some kind of mapping or transformation into the VE to make the data presentation more understandable. In any case, the items represented in the virtual world are collections of polygons and 
pixels (described by files) that are rendered to produce three-dimensional images. Some ability to manipulate or interact with the computer generated objects is generally associated with VEs although this does not have to be the case. VEs applications may have the capability to immerse the user in the scene by using head-mounted stereoscopic displays.

The general, full-featured computer-based SBPD system described above does not exist at present. Researchers have made, however, considerable progress toward developing parts of an integrated system in bounded domains such as ship and airplane designs. Goals of these systems include incorporating the features needed so that design concepts and evaluations can be made mathematically (through simulation) without constructing large-scale physical models and prototypes. The Advanced Research Projects Agency, for example, is examining and developing the SBPD concept for large Naval ship building tasks. Boeing is also using aspects of SBPD for the design and simulation of new aircraft designs such as the 777. Eliminating model construction is expected to be a considerable cost savings in the long term for such large design and construction applications. The ability to make subtle changes in design features on large, costly, design concepts or equipment and then evaluate the performance impact of such changes rapidly without physical model construction will constitute a powerful capability.

Many training capabilities and advantages are associated with the SBPD concept. Flight training simulators have been successfully used for pilot training for many years. Given the capabilities associated with the a full-featured SBPD system, simulators may eventually be available for arbitrary equipment types and physical systems. Such a training system would allow planners and equipment operators to define and practice equipment operations in a simulated environment before effecting the operations in the real situation. This training would help to reduce and communicate risk for hazardous tasks such as critical crane lifts and waste remediation operations.

For the simulation and training capabilities to be effective, the environments or computer-generated worlds must be defined. For some cases, definition of these worlds may, in principle, be straightforward. For example, in situations where the environment is not changing and can be readily described (such as the layout of a building) or prescribed (such as flight paths for a flight simulator), a computer-generated world can be constructed even though it may be tedious. In other cases, the environment of interest may be less well specified. Buried waste is such a case because, at present, there is no single method or technology that has the ability to reconstruct the landfill characteristics. Various research and technology development efforts are currently being carried out within the Buried Waste Integrated Demonstration (BWID) Program to develop, examine, and test techniques for gathering information that will be useful for characterizing of subsurface waste sites. Such efforts include using and improving surface geophysics methods for buried waste characterization (Coleman 1993), digface characterization (Josten 1992), and remote system characterization (Sandness et al. 1993). These efforts have obtained or will be obtaining data from many different types of sensors and instruments. Each of the techniques will have advantages and disadvantages for a given set of conditions and circumstances, and disparate types of data will be available. Clearly, there is need to determine how to extract information and proper insights from these available data and how to present this information to the end user to maximize the knowledge gained.

\subsection{Technology Need}

Remediation of buried waste in the U.S. Department of Energy (DOE) complex is currently and will continue to be a challenging technical and political problem. The challenge stems from the lack or inadequate characterization of and potential health and safety hazards associated with the waste sites. 
Need-driven approaches have been used to identify technologies for implementation in DOE's environmental restoration/waste management, and decommissioning and decontamination activities. The needs included identifying better (i.e., faster, safer, cheaper, and efficient) methods to characterize, evaluate, test, and demonstrate retrieval technologies. The concept of state-of-the art visual technologies, such as scientific data visualization, VEs, and SBPD was included in the assessment. Of particular interest is the desire to determine, evaluate, and communicate risk during any remediation process. Technology that provides ready access to and improved interpretation of all types of information (e.g., characterization, historical) available for a site under consideration will help assist in the determining and communicating risk. Advanced visualization and VE techniques can play a role in this effort. 


\section{DEVELOPMENT OBJECTIVES}

This work was designed to define the feasibility of VE techniçues used in conjunction with advanced visualization techniques to improve understanding of characterization data. This included development of a hardware and software platform for integrating diverse techniques to support improved understanding and presentation of information. The work was the initial start at defining the feasibility of and the opportunities for the use of VE display techniques.

To achieve the overall goal of defining feasibility and opportunities for the use of VE techniques, the following specific objectives were defined:

1. Develop a rapid prototype for soliciting user ideas

2. Define functional requirements for a prototype system

3. Develop a hardware and software prototype platform based on defined functional requirements.

Based on the early evaluation of the rapid prototype system, additional objectives for development in fiscal year (FY)-94 were defined:

4. Develop depth-to-object interpretation algorithms and automate these for use in digface data interpretation

5. Investigate the applicability of an automatic target recognition (ATR) system (previously developed for a separate use) in interpreting data from digface sensors to define waste types or objects

6. Investigate the applicability of neural networks for interpreting data from digface sensors to define waste types or objects

7. Investigate the applicability of a visual data base for presenting historical records and possibly integrating it with sensor data for improved understanding of available information on waste sites

8. Investigate the use of immersion as a tool for operators or decisionmakers to improve understanding of characterization data

9. Investigate potential product commercialism and factors affecting marketability of project results.

This work was closely integrated with the Digface Characterization Project (Josten 1995) at the Idaho National Engineering Laboratory (INEL). The digface project has and continues to gather some of the most advanced, high-resolution site characterization data fior engineered waste sites. Also, the digface project members have considerable knowledge, insight, and experience about the issues and needs for characterization data interpretation, analysis, and display. A particular focus is on supporting digface remediation operations by providing the operators and support personnel with rapid and effective 
presentation of information derived from the digface sensors to aid in rapid and safe decisionmaking during operations. Because of the dependence on the digface data, a brief description of the digface project is given in Appendix A. 


\section{QUALITY PROCEDURES, HEALTH AND SAFETY, ENVIRONMENTAL OVER'VIEW}

\subsection{Quality Procedures}

The VE project work consisted of data display and interpretation techniques implemented in software. At current stages of exploratory development, no specific quality procedures for the software have been implemented. Standard engineering practices were used, and manual verification of results in certain applicable instances was conducted. Detailed verification and software configuration control were not appropriate at that stage of development. Work consisted of analytical modeling and computer software development. There were no health, safety, or environmental concerns associated with conducting the project work. 


\section{PROJECT DESIGN AND DEVELOPNIENT PHILOSOPHY}

The project was structured to meet the objectives of defining the feasibility and determining opportunities for VEs in buried waste characterization. A rapid prototype environment was developed as a basis for generating ideas on potential applications. This environment was shown to potential users such as geophysicists and Environmental Restoration personnel for their input on desired capabilities for a prototype system. Based on the feedback received, a set of functional requirements was written, and a prototype software and hardware platform was developed. A number of separate self-contained analysis techniques for supporting data interpretation and display were developed and evaluated for potential use.

The system being developed has an architecture that can incorporate different software techniques and hardware if appropriate. Figure 4-1 shows the overall design, depicting an integrated system that has access to data, data base information, and analysis tools for buried waste characterization applications. The elements shown in Figure 4-1 are described in more detail in Section 5.3.

The philosophy of the project has been to leverage, to the extent possible, equipment, knowledge, techniques, and software from other sources. This philosophy resulted in some sharing of costs with INEL General Purpose Capitol Equipment funds for equipment. Some vendors provided software and hardware products at no (or little) cost as they felt future benefits could be significant. Other private concerns provided insight and shared ideas and enthusiasm for the project. The need for commercialization was recognized early in the project.

Efforts to remain abreast of developments ensured that duplication was minimized and other research contributed to or benefited from this project.

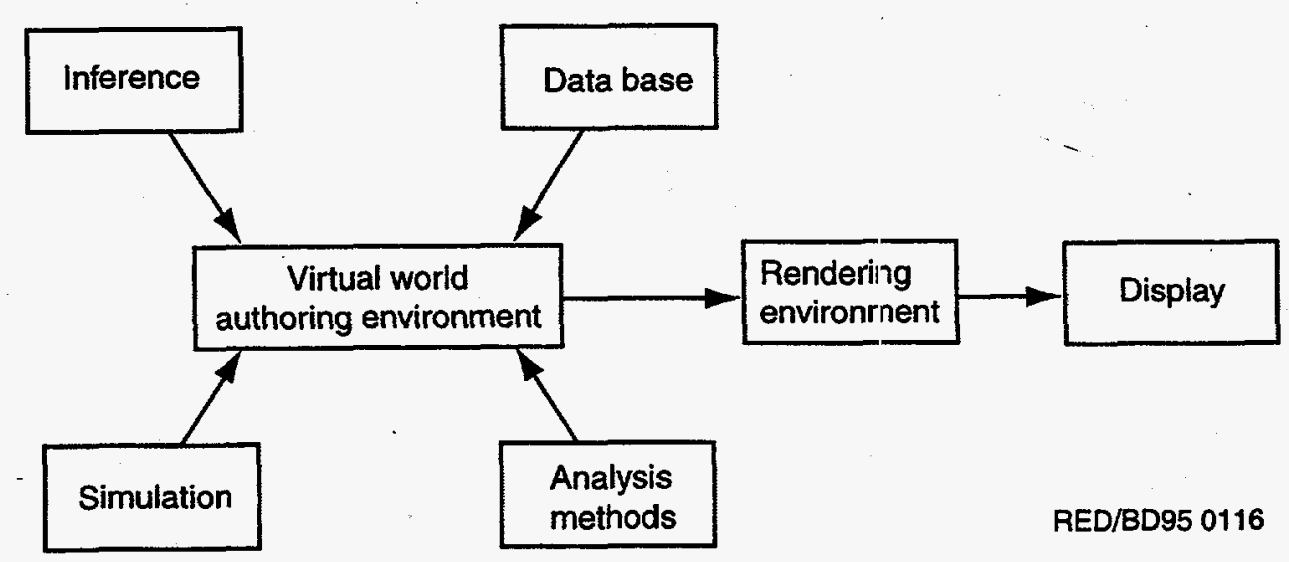

Figure 4-1. Overall design schematic of VE system. 


\section{RESULTS AND PRODIUCTS}

Rapid prototype environments were developed to solicit user input and define requirements for this project. These environments, the resulting functional requirements, and work conducted to address the functional requirements are described below.

\subsection{Rapid Prototype Environments}

The rapid prototypes provided baseline environments and when shown to potential users could communicate current capabilities and solicit input about functionality. The Characterization Cell in the INEL's Cold Test Pit (CTP), was used as a basis for this work because this engineered facility is well defined and significant engineering and geophysics survey characterization data are available.

Three rapid prototype environments were constructed: a walkthrough of the Characterization Cell, a visual data base of the Characterization Cell, and an example three-dimensional data display. These environments were shown to and discussed with potential end users including geophysicists, engineers, and environmental restoration representatives. These environments and conclusions reached are described below.

\subsubsection{Walkthrough Models}

Three-dimensional walkthrough models of the Characterization Cell and of the entire CTP were constructed using a PC-based commercial software package called VIRTUS (VIRTUS Corporation 1993). VIRTUS is typically used for computer-aided visualization for spatial design applications. The software application includes a sophisticated modeling and Rendering Environment (RE). The modeler includes a full suite of modeling and lighting tools. Geometric models are constructed in two-dimensional planes and extruded into the third dimension to produce three-dimensional objects whose surface features can be textured or altered as desired. The objects comprising the model are rendered in a separate window. The user moves through the rendered view as desired to look at: any aspect of the model from any viewpoint. User movements are recorded and played back at a later date or saved as a movie for playback. No user interaction with the model and no hooks to the external world are possible.

The VIRTUS model provides an example of a reasonably high visual fidelity modeling environment. The environment is useful for lighting and spatial analysis if the geometry of the objects comprising the world is known. The model of the Characterization Cell was usefil for demonstration purposes in that it showed what was possible in a VE given complete definition of the geometric features comprising the world. Figure 5-1 shows a screen image from the VIRTUS model of the Characterization Cell. This view direction is from the northeast to the southwest direction. For example, if it was desired to reconstruct a three-dimensional image of a waste site after retrieval

a. G. G. Loomis, "Design and Construction Details of the INEL Simulated TRU Test Pit," EG\&G Idaho, Inc., Engineering Design File, BWP-150-009, March 2, 1989.

b. M. R. Winberg, "Specifications of the Addition of a Characterization and a Retrieval Cell to the Cold Test Pit (As-Built)," EG\&G Idaho, Inc. Engineering Design File, December 29, 1992. 


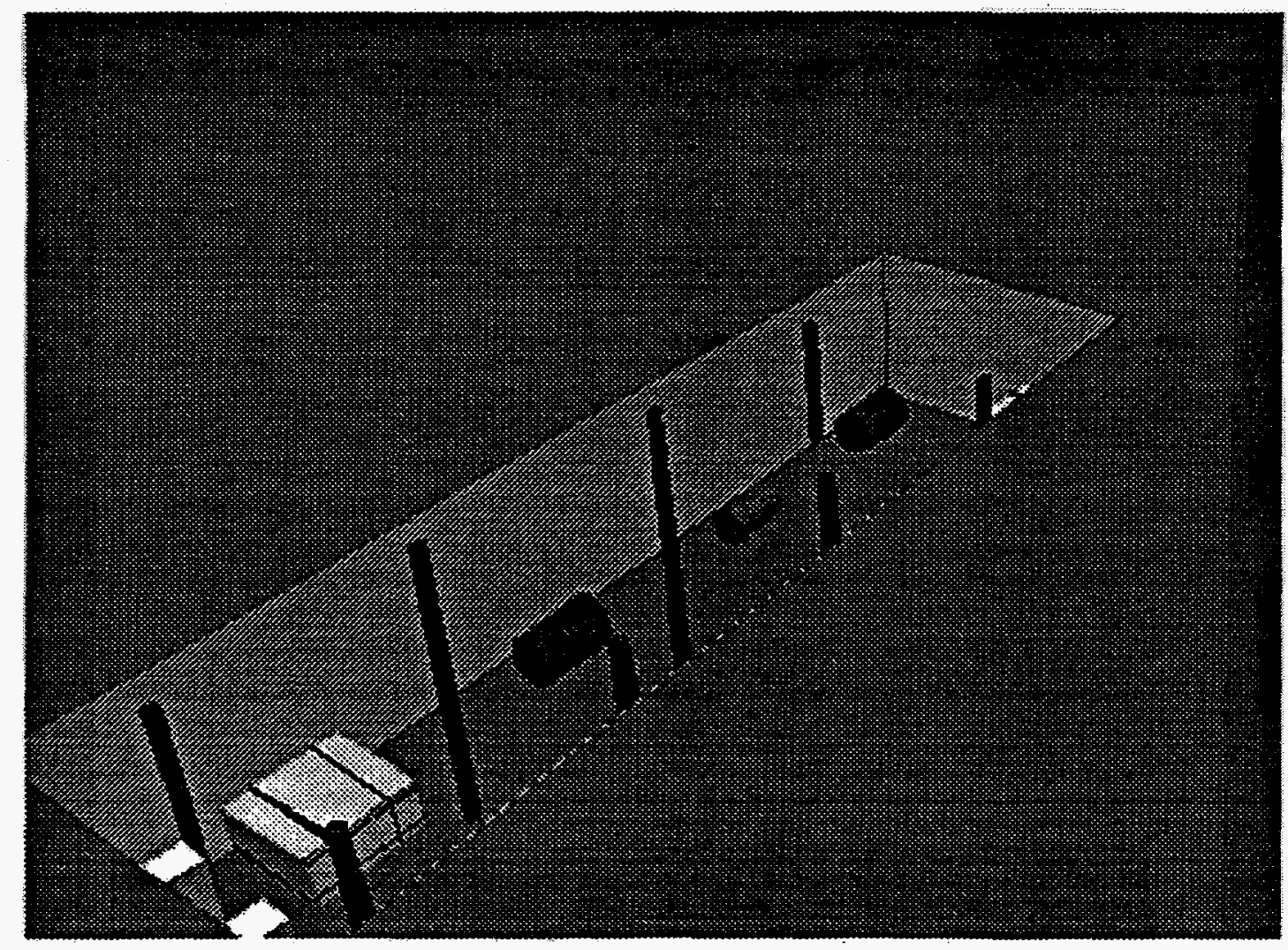

Figure 5-1. VIRTUS screen image of CTP model (northeast to southwest view).

had occurred, such a model might be constructed using data from retrieval records to provide historical documentation of waste location. However, since interaction with the model elements and communication with the outside world is not possible, no means of providing additional information about particular items of interest is possible. This limitation is characteristic of the VIRTUS package. Other software tools have additional capabilities beyond this.

Conclusions reached from review of this VE suggested that walkthroughs could be useful under certain circumstances. For example, if a plan existed for the design and construction of some new site, component, or facility, the site specifications (e.g., dimensions, location) are reasonably defined at least in a preliminary sense. A three-dimensional VE model of this situation can readily be constructed to address questions such as "how will the site look," "will the lighting be adequate," "is the design adequate in terms of accessibility for vehicles, personnel, and machinery," or "does this component block that component in an unacceptable fashion"? For waste sites, this kind of environment could be a useful tool for asking those and related questions, provided the site is fully documented so that a model can be constructed. While this is typically not the case for buried waste, stored waste removal and retrieval could find utility in such modeling. A possible application of walkthrough type modeling would be in the generation of a model after or during the excavation, retrieval, and remediation of a buried waste site. This would be a method of cataloging and documenting the site after the fact to determine and/or preserve relevant specifics and to answer "how the site looked." Preservation of such information in this form might be useful for future analytical modeling efforts, for communicating information to other parties, as a training set for operators, and for supporting learning by experience processes. Preretrieval survey 
data and postretrieval information, for example, could be analyzed to enhance understanding of what the survey data are telling us and help synthesize relationships or patterns that can be of future use.

Accurate cataloging and documenting of retrieved waste seens to be an important area. Although this project is not actively pursuing this concept at the moment (work to be discussed later using the Pit 9 data base is relevant), we feel strongly that design consideration should be given to this area. For the reasons cited above, it seems that a method where the relevant specifics (perhaps waste classification, depth, survey data, graphics images) and metadata (text documentation) of retrieved buried waste can be preserved in a useful fashion would be invaluable for the historical record. A method that has access to and links between the significant information obtained before, during, and after a buried waste characterization and retrieval operation would seem to be an supert organizational tool. For discussion, one might consider such data types as photographs, videotapes or video clips, geophysics data, written operations logs, assay results, procedures, and records of decision. As a point of information, the National Center for Supercomputing Applications Mosaic (NCSA Mosaic 1994) software product has some of these mentioned capabilities where links between documents (graphics, text, and so forth) can be established using hypertext and hyperlink protocols. The documents may be on one computer or distributed across a network of computers. Mosaic has been used to a limited extent to organize some of the digface characterization data. It seems that the data to be gathered during the FY-95 integrated demonstration or the retrieval of Pit 9 would be an excellent opporiunity to examine this issue and could be explored as part of the FY-95 workscope for this project.

\subsubsection{Visual Data Base Model}

A visual data base model of the Characterization Cell was constructed using the commercial software package VREAM (VREAM, Inc. 1993). VREAM is an object-oriented environment that runs on PCs (IBM PC compatible only) and is a general purpose package used for interactive world construction. A three-dimensional modeling/editing environment and a separate runtime interactive environment constitute the system. Models are generally constructed in the three-dimensional editing environment and then transferred to the runtime environment for interactive use. Objects or groups of objects in VREAM can be assigned attributes such as color, translation, rotation, animation, or inertia. Significant capabilities are provided for links where an action instigated by the user or an action taken on or by one object (e.g., movement, touch) can cause an action (e.g., movement, display of information) in another object or group of objects. The VREAM software also capable of communicating with the outside world. Calls to external programs can be made and results can be communicated back to the VREAM model. Models constructed in the VREAM editing environment generate script files that can be read and easily modified by an experienced user. The software also includes drivers for numerous alternate input, tracking, and stereoscopic viewing devices.

The VREAM visual data base model constructed of the Characterization Cell consisted of the geometric objects known to be located in the pit along with a ground plane as shown in Figure 5-2. Waste forms were manipulated and touched or grabbed using the interactive pointing device (a virtual hand in this case). Grabbing the a waste form activated links that highlighted the object and provided a tabular listing of information about the object including identification number and contents of the container. These links could be far more sophisticated. For example, a link could cause activation of an external program (i.e., a data base, a spreadsheet, or some other executable program) that could return information to the screen or pass variables or information to and from the object activating the link. Appendix B provides VREAM screen images of the CTP model illustrating some of these features. 

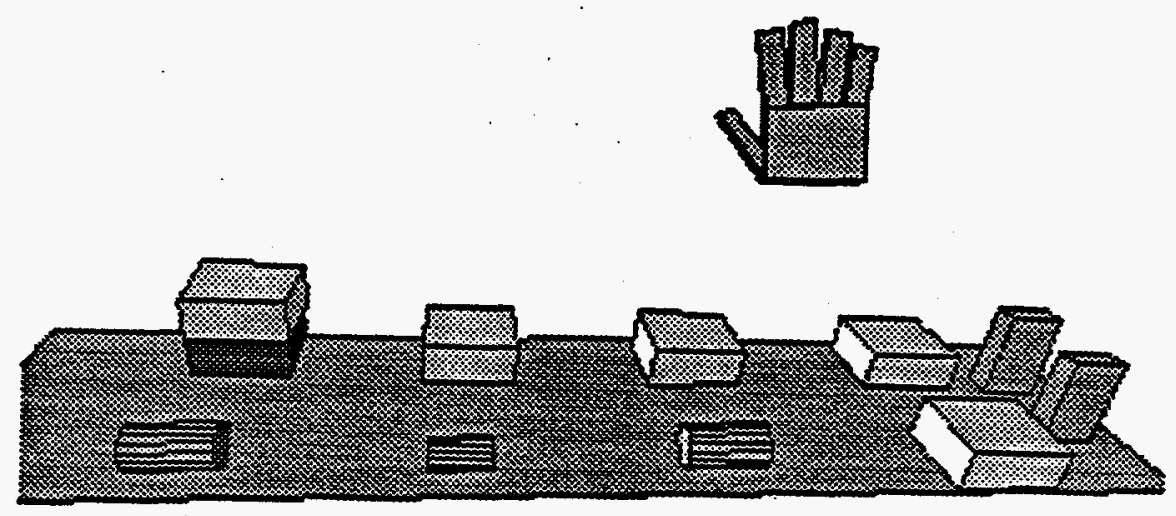

Figure 5-2. Screen image of VREAM model of CTP (south to north view).

The VREAM prototype model can be considered a visual information manager similar to that reported by Rod (1992) for stored waste. Such a system would have immediate utility for circumstances where waste is stored in an orderly fashion, the waste forms are known, and organized information about the waste (content for example) is available. A three-dimensional environment like this would provide all types of information for use in communication, interpretation, and planning.

The visual data base was an interesting concept to people reviewing the prototypes. The following observation was made regarding Pit-9, "The pit dimensions and volume are known and the best available information indicates that certain volumes of particular waste types (e.g., pallet of barrels, pallet of boxes) were placed or dumped at particular locations in the pit. However, the placement locations were worker eyeball estimates relative to pit stake markers. Thus, there are discrepancies and conflicts relative to how much waste volume can be located at certain positions in the pit relative to somewhere else in the pit." One manifest indicated a load was placed at some position, but another manifest indicated that all or part of that position was already occupied. There was interest in the concept of a three-dimensional VE model that could be used to examine "what-if" scenarios about the positioning of known waste shipments of known volume in the pit. Interest was also voiced in conducting "touchability analysis" and investigating the possibility that one or the other of the shipments was actually placed at a slightly different location, and after doing this, seeing if the expected placement makes sense in relation to available survey data. This analysis could help with determining accessibility. For example, given a set of conditions, one may be able to assess spatial clearances and estimate if retrieval equipment can get between expected storage areas without disturbing undesirable locations or conduct other types of retrieval planning.

A visual data base model would be useful for the problem described above if there is sufficient documentation to build crude models. Under these conditions, the model would only be as useful as the information available to construct it. The visual data base could also be used for cataloging the results

c. Doug McKenzie, Idaho National Engineering Laboratory, personal communication, January 17, 1994. 
of a digface or other remediation operation. Using excavation records, a model reflecting the waste removed could be constructed and the model elements linked to more detailed information about the remediation (e.g., survey data, shipping records) residing in data bases or elsewhere. Detailed records of retrieval operations would have to be maintained to implement this type of documentation tool. Potential benefits are proper ties between preretrieval data, and actial retrieval results could expedite the learning process and facilitate improvements in data interpretation for future operations. While cataloging and documenting retrieved waste is an important issue, this project is not pursuing its development. Work with retrieved waste cataloging has been done using the Pit 9 data base, but this area deserves additional attention.

\subsubsection{Three-Dimensional Data Display/Analysis Environment}

Data from the Digface Characterization Project became available in late December 1993. These data constitute three-dimensional data sets gathered from several different sensors over the digface surface. As discussed by Josten (1995), analyzing the data using PC-generated, hardcopy, two-dimensional plots was a frustrating, time consuming process that did not facilitate trend analysis between data sets of the same sensor type or between data sets of different sensor types.

Data from digface Experiment 1 (Josten 1995) were used to develop a prototype three-dimensional, interactive, multivariate data display environment using Advanced Visual Systems (AVS) software (AVS 1993) and running on a Sun workstation. Display environments are created in AVS via an interactive, visual programming interface that allows code "modules" to be graphically linked in an object oriented sense to accomplish desired tasks. The modules include code that can read data sets, perform operations (e.g., filter, reorganize, search for duplicate entries) on the data, color enhance, and display the data. Many code modules are available in the environment, and users can write their own modules to accomplish desired tasks. Representations of physical objects (e.g., barrels and boxes) can be imported into the display environment along with the data display to provide visual cues. This environment, while not optimal, was generated to examine methods of displaying ard manipulating the data sets and to provide information to the user.

A volume data set of information from the four parallel planes gathered during Experiment 1 was constructed by locating the individual data sets. An object representing a single barrel was also included in the environment as shown in Figure 5-3. This prototype allowed the user to interactively rotate and translate the data set and to examine it from arbitrary points of view. Contour line and color gradient fill maps could be viewed on various planes cut through the data set. Certain features could be made transparent (or temporarily removed) to provide better visual access to enhance tracking the features from one data plane to another. Visual enhancement techniques ccould be examined including bubble visualization, opacity mapping, color coding, symbol typing and sizing to display different data types and magnitudes, and constant value surface display. Such a display environment with the proper control and manipulation capability would allow the user to search for data relationships (Josten 1995). Searches could be conducted in a more efficient manner than currently practiced by providing access to tools that search display data in many ways and use visual cues in the process. This capability will support analysis and help identify, classify, and characterize objects. 


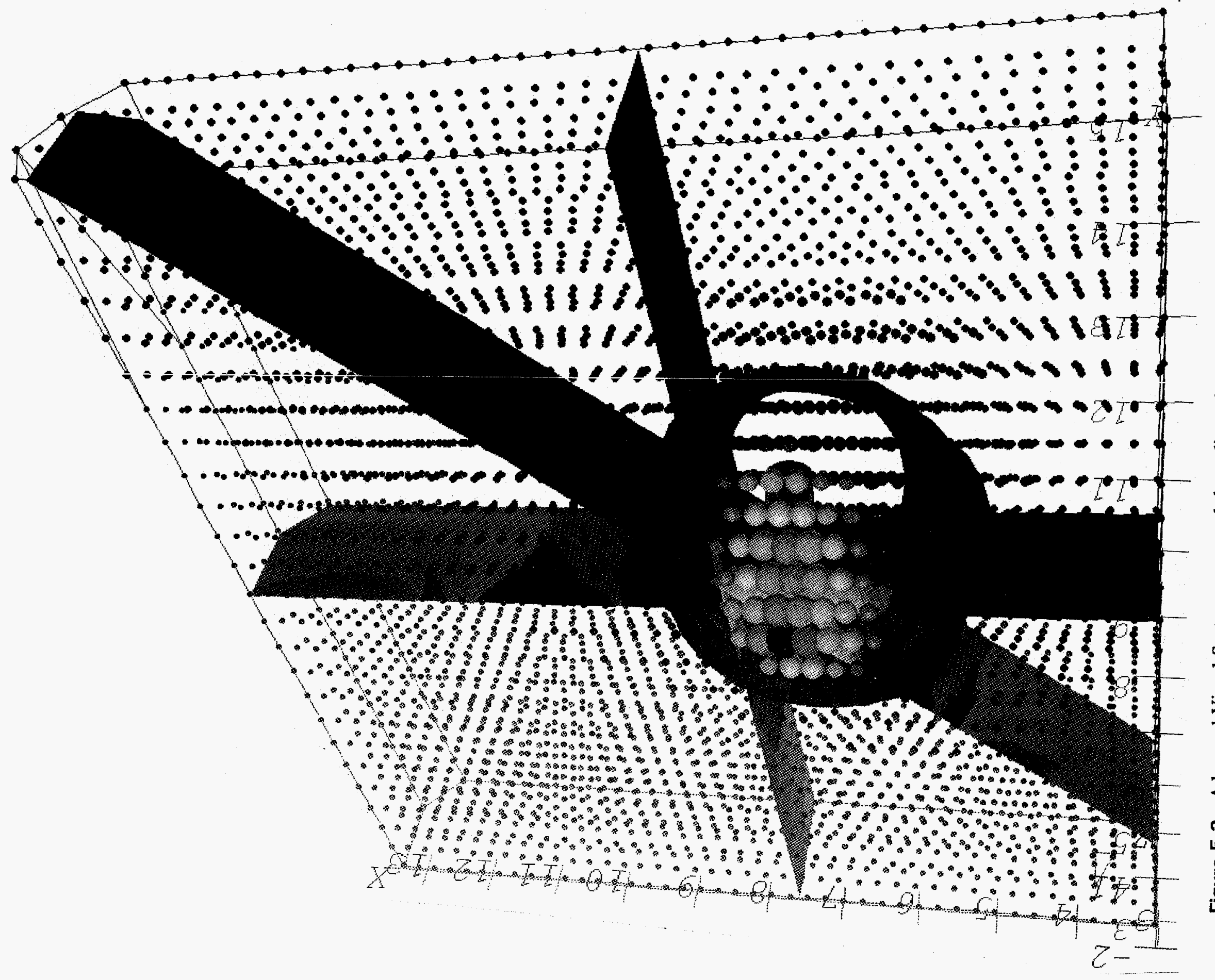





\subsubsection{User Feedback On Rapid Prototype}

Positive and negative input was received on the prototype data display environment. A typical comment was that the large amount of information caused confusion. This was expected because the data were not presented in a way people were accustomed to. The confusion can be reduced by iterative display design, selectively displaying information of interest, and removing information no longer needed. The environment must provide what the current user is interested in, whether it is an operations person interested in newly acquired data, or an analyst or geophysicist interested in reviewing new data along with previously acquired data sets.

From the view of digface data acquisition and operations, interest was shown in displaying data on-line in a manner superior to the typical flat two-dimensional line plots or profiles. Another comment made about numerical simulation results was if a library or data base of simulation results (not presently available) were readily accessible, could comparison of measured clata to simulation data for an assumed waste configuration be a useful decisionmaking aid? In theory this process is possible, but the large number of simulation configurations and circumstances (e.g., interference and superposition considerations) would make it an unwieldy process. Pattern or target recognition or feature extraction schemes (Lassahn 1993; Poulton 1992) may make this a more tractable problem. An example would be training schemes to look for a specific subset of commonly expected waste forms (e.g., types, combinations, or orientations of containers) using a combination of data from engineered mockups and simulation data. This issue will be discussed in more detail below. The Interactive Computer Enhanced Remote Viewing System (ICERVS) approach described in MTI (1993) could support and benefit from this information and the empirical identification techniques deriveil from data analysis.

Geophysicists indicated that there are several parameters of interest concerning buried waste. These parameters include the estimated depth, estimated volume, and composition (e.g., metallic, nonmetallic, hazard potential) of the waste. Empirical techniques show promise for estimating some of these parameters based on the digface characterization and other surface surveys. The geophysicists also indicated that a display/analysis environment that could incorporate and automate applying the empirical techniques and others to be developed would be useful for characterization analysis. They indicated that a useful tool would be the ability to easily superimpose, view, and nanipulate experimental data gathered over buried waste objects of known type and location with representations of those objects. The expectation is that visual cues provided by spatially correct three-dimensional representations of the buried objects relative to the respective data would be an asset. It was clear, however, that the geophysicists wanted to view the data in a familiar manner (e.g., contour line plots or surface plot), where they could apply "domain rules" learned through experience. They also stressed the need to start simply and progress to more complex approaches as more is learned about the data relationships. While the display must identify boxes, barrels, drums, and large objects, it must also highlight potential hazards such as chemical radiation.

\subsection{Functional Requirements Definition}

Based on the rapid prototype environment reviews, the following conclusions were reached about functionality of an environment to support the stated needs of the reviewers.

- The environment must accommodate and use large layered data sets that will be encountered in digface characterization/remediation type operations. 
- The environment must accommodate multivariate data because of the variety of sensors that may be involved in digface operations.

- The environment should be able to handle data gathered on, or representative of, a wide variety of physical coordinate spaces.

- The environment should be capable of displaying information in a variety of ways because of the diverse range of potential users.

- Traditional techniques should be available for scientific visualization across a wide range of disciplines to provide all users with a common set of tools. Users from specific disciplines should also be able "see" the data in a manner to which they are accustomed.

- Traditional methods should be included for displaying geophysical data that lie outside of those normally used in the visualization process.

- The environment should provide the supporting structure in which domain experts may conceive, implement, test, and refine new empirical algorithms for description, identification, and classification as may be warranted by their perception of the data.

- The environment should provide visual cues for patterns and features detected by either the analyst or system. For example, once the determination has been made that an object of a given type is probably located at some point, a graphical representation of the object may be rendered at its probable location in three-dimensional space.

- The environment must be extensible so that new empirical algorithms, display routines, simulation capabilities, or other components can be added without major restructuring of the hardware and software.

- Given the large amount of data, the environment will need to incorporate a data base of increasing complexity.

- Finally, a reasonable level of user control is necessary to make the system efficient for the user. High performance hardware and software is one method to provide adequate system performance.

- Performance must be available that provides a reasonable level of interactivity.

These conclusions suggest a parallel path development approach including integration of software/hardware components and developing of digface data analysis/interpretation and display tools to support the user's needs.

\subsection{Development of Integrated Software}

The design of the integrated hardware and software platform was based on the functional description of the necessary analysis, archival, and user interaction capabilities (see Figure 4-1). These requirements were based the ability of the operator to interactively manipulate any reasonable subset of the available 
data in real time. The data under investigation must be easily and readily accessible for analysis, manipulation, and display by the user. In addition, the methods and results of past analyses should be stored to provide a knowledge base for future activities.

The functional design consists of seven components; five are software and two are hardware (see Figure 5-4). The specified components are available off-the-shelf. As faster, more flexible, or superior components become available, they may be integrated into the system at minimal expense and rework. The simplest to implement and describe are the hardware components in a RE and the User Interaction Environment. The software units are designed to accomplish the tasks identified in the requirements analysis. This is generated functional software units for data analysis, representing and manipulating the virtual world, semiautonomous data analysis, access to external simulation capabilities and a high performance, distributed scientific data base.

The choices for platforms to provide the rendering capabilities were limited to scientific workstations with special purpose graphics hardware. This limitation was due to the complexity of the environments described and the need to provide reasonably high frame rates to enhance the perception of immersion. At the beginning of the project, there were three potential vendors with solutions. The final choice was based on price/performance, breadth and quality of available software, stability in the workstation marketplace, system expendability, flexibility, reliability, and sharing of costs with other funding sources.

The system acquired was a Sun Microsystems SparcServer 2000 with eight $50 \mathrm{MHz}$ processors each with $2 \mathrm{MB}$ of external cache. The system contains dual, packet-switched communication buses that allows memory accesses to be spread over both channels. The system is capable of expanding to 20 processors, currently running at $60 \mathrm{MHz}$, but soon will include $80 \mathrm{MHz}$ and faster processors. The system is also configured with four Fast/Wide Differential SCSI disk controllers capable of sustaining $12 \mathrm{MB} / \mathrm{sec}$ to and from disk space. Currently the system has four disks and is capable of approximately $12 \mathrm{MB} / \mathrm{sec}$ total throughput. There is also a tape backup unit and CD-ROM drive on a conventional SCSI-2 interface. For communication to other machines, there is a $10 \mathrm{Mb} / \mathrm{sec}$ Ethernet controller and $100 \mathrm{Mb} / \mathrm{sec}$ FDDI interface.

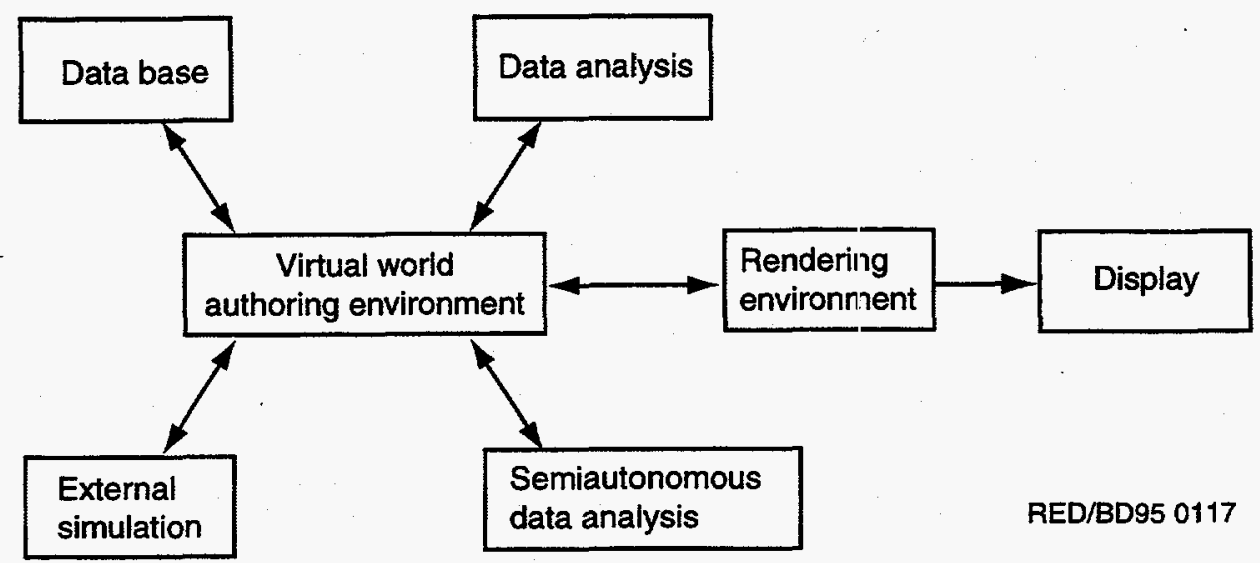

Figure 5-4. Functional design diagram. 
The graphics framebuffers currently being used are one eight-bit GX for the system console and two hardware accelerated 24-bit ZXs. The ZXs were chosen for their ease in integration and relatively low cost. Although the $\mathrm{ZX}$ performance is approximately one-fifth that of the best available, 210,000 polygons/sec versus $1,000,000$, framebuffer technology is still maturing and the next year will bring great advances in performance and price. The main drawback to the $\mathrm{ZX}$ framebuffer is the lack of support for hardware textures. While this is a considerable disadvantage in traditional immersive applications, in the present applications it has little importance.

Normally, the number of polygons in a scene is minimized to increase the frame rate. To make up for the larger polygon size, textures are employed to provide a greater sense of reality. With the datadriven representations of the objects displayed in this system, accurate geometries are more important than the surface colors and characteristics usually modeled or enhanced with textures. The system could be built, tested, and evaluated without the significant additional expense of state-of-the-art framebuffers (usually one-third to one-half of the total system cost). If testing of the existing shows that frame rate should be increasing, the high-end framebuffers may be added transparently to the system.

Key to any immersive system is the manner in which information is communicated between the system and the user, otherwise known as user interface input/output. For image output, a high resolution head-mounted display (HMD) was chosen primarily for the ability to free the user's hands. This allows a more natural interface between potential users and the VE. The specific HMD was selected because of its comfortable use and relatively high resolution. While most HMDs are limited to 8 bit color with $300 \times 200$ pixels at $30 \mathrm{~Hz}$ interlaced, the $\mathrm{n}$-Vision $10 \mathrm{x}$ is capable of $640 \times 480$ at $60 \mathrm{~Hz}$ noninterlaced or $1280 \times 960$ at $30 \mathrm{~Hz}$ interlaced, all in 24 bit color. This device will not only enhance the sensation of immersion due to the higher fidelity images but will increase accuracy because the operator will be able to better resolve small features. The HMD position is determined by a Polhemus Fastrack system, which provides six degree-of-freedom information with respect to the helmets position and orientation. The HMD also includes stereo headphones, and the computer system is capable of generating any required audio signal. Sonification and three-dimensional sound have not been included in the software of the existing system as they were not specific user requirements. These components can easily be included if they are needed in the future.

User input was limited to the traditional mouse and keyboard for the present implementation of the system. The type of operator the system may eventually encompass has not been determined; it is difficult to determine that user's need for interacting with the virtual world. The software can support almost every commercially available input device, so implementation of a more refined user input environment will be straightforward once the requirements have been determined.

The core of the software components in the Virtual World Authoring Environment (VWAE) is currently the WorldToolKit (WTK) (Sense8 Corporation 1994) product from Sense8. WTK was chosen partially because of its ability to run on a wide variety of computing platforms, from the highest-end Silicon Graphics Reality Engine2, Evans \& Sutherland Freedom, and DEC/Kubota Denali to PC compatibles under Windows and Windows NT. The package consists of a library containing a series of C-callable functions, which provides flexibility and extensibility. This software is the controlling process in which objects are created, manipulated, and displayed to the user. From this application, hooks are built into the other software components, the data base, the data analysis, and external simulation and semiautonomous data analysis. Also, Sense8 was willing to perform a series of specific modifications to their existing product to optimize it for the hardware environment under construction. 
Sense8 provided a new version of their WTK product that incorporated multiple framebuffers and multiple processors support. This allowed the system to make maximum use of the available resources. In this model, one framebuffer drives the CRT for each eye. The stereo images are generated in parallel, providing an increase in frame rate. Each framebuffer resides on a system board that contains two processors, and those processors are dedicated to providing the polygon lists that will be rendered into the images viewed by the user. The other system processors are available for data base access, data processing and analysis, and general system functions, such as processing user input.

The second software component is the data archiving subsystem shown as the "Data base" component in Figure 5-4. This subsystem must be capable of storing, searching, and retrieving a wide range of data types from an extremely diverse set of data sources. Available historical data are included as well as whatever metadata can be collected. So descriptions of what was thought to have been buried as well as information concerning the contents, form, and origination are available. Any site characterization data should be available within the system as well as the results and methods from previous analyses. An operator should be able to request of the system, "What types of analysis has seemed to work successfully on this type of data in the past, under similar or closely related circumstances"? While the data base was not capable of responding adequately to the previous request, it is the goal to which the system is striving and the one that was considered in the system design. To process such a request, previous analysis procedures will also need to be stored in the data base, so that such questions may be asked. The current data base product is the Aurora Data Server from Xidak. This is a multiuser distributed data base that is accessible via a C-callable library and the SQL command set. In this fashion, previously successful analysis procedures may be called up, virtual world scenarios revived, simulation parameters or results restored, and/or advantageous situations for the semiautonomous data analysis routines recalled.

For the more traditional, interactive scientific data analysis processes, there needs to be a modular software toolkit from which analysis procedures can be constructed. There are several of these visually programmed scientific data analysis toolkits on the market today. The one chosen for this project is the AVS (AVS 1993). AVSs runs on all major Unix scientific workstations, has the largest user base, and has a large number of user contributed modules available at anonymous ftp sites ${ }^{d}$. It is flexible and easily extensible, includes a machine independent data transfer protocol, utilizes shared memory for efficient use of machine resources, and can operate as either client or server for distributed processes. In its newest incarnation, AVS will be available on PC compatibles running Windows NT.

This provides the user with traditional visualization capabilities as well as the ability to easily add new techniques as required by users. Most two and three-dimensional visualization capabilities are included in the module set. Line, scatter, and other types of plots can easily be generated as well as relevant axis and label information. A large variety of three-dimensional representations can be used including cut planes, isosurfaces, contour lines, and volume dicing. These can be used either alone or in conjunction with the others. There are currently five different types of volume renderers available within AVS, allowing users to choose the one that best matches their needs, the problem complexity, and the capabilities of the computing platform.

d. Anonymous $\mathrm{ftp}$ sites are computers on the network that can be accessed (without having an account on the particular machine) to download software. 
A real advantage of this system is the ability to handle unstructured data directly, without having to resort to gridding in order to process and display the data. This reduces the number of times the data are filtered between the collection and display and increases confidence in the resulting images. The available data are most often not on what is referred to as a regular grid under the more controlled situations of site characterization. With the real-time field conditions of a digface-type operation, there is little (if any) hope that the data will be uniformly sampled or collected. The capability for dealing directly with unstructured data becomes paramount in these conditions, which are those most likely to be faced by an operator, and for which it is highly desirable to reduce the risks of exposure.

Data from the data base can be used as input to the AVS networks and the geometric results can be passed to WTK for user display. Current implementation has the generation of geometries occurring as a separate step, but the next step is to incorporate this functionality directly within the central WTK process. Additionally, once a successful and useful network of modules is determined, this network may be saved within the data base along with related keywords and metadata. This will allow retrieval of the network for a future problem of a similar nature. As AVS does not care where input data comes from, the data source may be existing numerical data within the data base, external simulation data, or real-time data from a data acquisition system. This makes the system useful in a wide range of scenarios including mission rehearsal and operator training, data analysis refinement, direct operator assistance, and feasibility studies.

The semiautonomous data analysis routines form the fourth component of the software system (shown in Figure 5-4 as semiautonomous data analysis). There are a couple of methodologies being investigated currently. They include the ATR system discussed in Section 5.5, neural networks described in Section 5.6, and depth-to-object discussed in Section 5.4. This technique is being implemented in two of the software components, both as an automated technique where the computer attempts to pick good locations for analysis and as a software tool under the data analysis component in Figure 5-4. In the latter case, a user will be able to choose the points to investigate via the depth-to-object methodology. A hybrid technique is also under consideration, where the computer makes a large number of estimates and displays the results to the user. Figure 5-5 shows a schematic of how such an interface might look. The user would then determine which of the many results had high degrees of confidence and select those for further analysis such as statistical approaches. The functional design and implementation of the system will support as many of these types of approaches as can be validated. As additional methods are established and verified, they can be added to the toolset available to the user. The information about the method along with its strengths, weakness, limitations, and areas of applicability can be stored in the data base so that they may be retrieved and used in other scenarios.

The final software component provides external simulation (the "External Simulation" component in Figure 5-4). This is implemented by the Portable Virtual Machine (PVM), a public domain library set from Oak Ridge National Laboratory. While the software library calls are currently in place, there has been no use of this capability to date beyond the testing and demonstration stage. The PVM routines allow data to be transferred in a machine independent fashion to compute server processes running on a distributed, heterogeneous network of workstations. In theory, this allows a detailed physical process to be simulated, such as the magnetic signature of a metal barrel, with the results passed back into the data analysis, virtual world, data base, and/or semiautonomous data analysis processes. This signature could then be compared, via these other software components, to that which has been observed. In theory, the magnetic signature of a large object could be computationally removed to reveal a smaller object that had been masked by the larger or nearer object. 


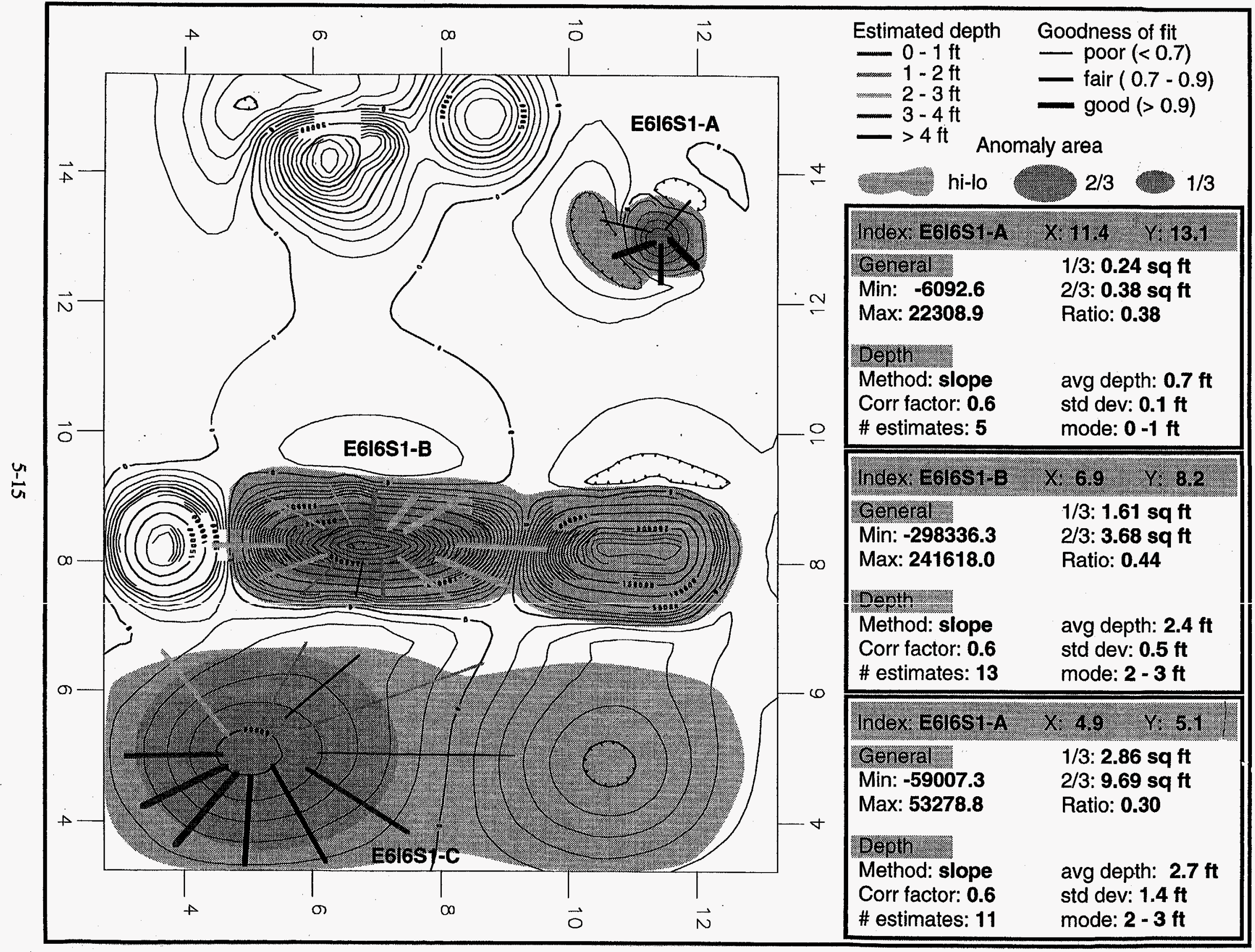

Figure 5-5. Schematic of how analysis interface might appear. 
In practice, it is not clear how reasonable the simulation described above is in the context of the current problem. Such physics-based simulations are among the most difficult computational problems. Presently, most simulations depend on knowledge of the structure and material of the object and surrounding media. These quantities are unknown in a waste site remediation effort, making an accurate analysis unlikely. Assuming such an analysis can be performed, doing so usually requires many hours on a supercomputer. The VE system is designed to work in the 10 to $60 \mathrm{~Hz}$ range, and results of such detailed physical simulations may not be available to the user within the time frame of interest. However, some simulations may be accurately performed in the near real-time operating environment of the VE generation system and access capability has been provided. At least one potential user of the system has shown an interest in this capability during the requirements generation portion of the project.

To provide for a functioning system, the interface requirements of the various components were significant issues from the beginning of the project. The functional requirements and design stage had to include the methods by which the separate components would communicate. The communication procedures have both hardware and software components.

The hardware system was produced with off-the-shelf components. The n-Vision HMD requires two synchronized video signals, each projecting the image as it would be viewed from user's eyes. This is accomplished by the ZX framebuffer programmable nature. New microcode was generated by Sun Microsystems to provide a compatible video signal with the HMD. The physical connections were handled by standard monitor cables and a 13W3-to-BNC pigtail. The HMD works by receiving a filed sequential color signal, which is a field of red, then green, and then blue for a single image frame. These fields occur at three times the $30 \mathrm{~Hz}$ or $60 \mathrm{~Hz}$ frame rate of the HMD. This was one factor that required new microcode for the ZX. The fields then drive a monochrome CRT within the HMD, which is viewed through an electronically controlled color filter, those being red, green, and blue. The resulting video fields are displayed so quickly that the user perceives it as a single 24 bit color image.

The software subsystem interfaces to the hardware through the system's SBus. The processors dedicated to feeding the framebuffers provide a string of optimized geometry known as triangle mesh strips. These t-mesh strips are produced by the WTK rendering software. The t-mesh strips are then rendered by the hardware of the framebuffer and output as a video signal to the HMD under the control of the ZX microcode. The VE user interface, such as head-tracking, is handled by the options available in the WTK libraries. All other hardware/software interactions are performed through calls to the Unix kernel.

Communication among the software components is more difficult due to the complexity of the data being communicated and the number of different components involved. The simplest interface is that to the external simulation capability. Since this is handled by PVM, data are simply repackaged into the appropriate buffers and their pointers passed to the PVM routines. Similarly, upon completion, the results are communicated by a pointer to the buffer containing the relevant information.

For the other components, the procedure is similar, although more complex. In most cases, the more general data format is chosen rather than a totally new, independent format. This reduces the data translation by a factor of two and works well with a small number of components. For the geometric information, such as that passed from the AVS modules to WTK, the AVS geometry format is used until the point where the structures are passed off to the WTK renderers. All WTK geometries can be represented in the AVS geometry format, so this provides an efficient mechanism for handling geometric 
data. Geometries input directly into WTK are usually in Autodesk DXF or Wavefront OBJ format. This provides the ability to use powerful external modelers to create geometries and maintain compatibility. The geometries are usually then converted to WTK NFF format for increased efficiency.

Floating point information is currently stored either in raw format, AVS unstructured cell data (UCD), or as AVS field data. The field data type is efficient and useful, particularly since it can be directly operated on by the AVS data analysis modules as can the UCD data. The UCD data type has more flexibility but often requires a significant amount of preprocessing to convert raw data to UCD format. Although this process is automatic, it can take minutes to hours for large data sets. The field data type occupies a middle ground, being fairly flexible and yet efficient to convert to and from. All three types, raw, field, and UCD, are currently used by the system. Ideally, a future implementation would be entirely based on the UCD format, which will also form the basis for future release of the AVS software.

Integration of various semiautomatic data analysis routines often require individual data translation. These routines have been developed, usually by outside sources for their own purposes and as standalone programs, and as a result should be wrapped within code to provide interface to the rest of the system. This wrapper code provides the translation to the AVS, PVM, or other required format.

The Aurora Dataserver implements a SQL interface for the issuing of queries. The information returned by the request depends on the nature of the request and the type of data that satisfied the request. These data have to be converted if not in the appropriate form, although much of the data will already be in one of the AVS formats, or have routines for converting to the format.

Currently, all the components are at least loosely coupled. The AVS data analysis is still performed external to the VE and the resulting geometries are then read into WTK. However, AVS itself can perform the VWAE role as well as data analysis. Data from the Aurora Dataserver can be read directly into AVS and then rendered in stereo to a workstation monitor, where users may view them by using the CrystalEyes stereo glasses. Existing historical and digface data are in the data base and can be requested by user-generated SQL queries.

The ability to construct and execute AVS networks within the VE still needs to be implemented. This will provide a more consistent interface to the user and reduce the number of separate steps that should be performed to analyze and display a given data set. The methodology for this implementation has been the subject of discussion for many months with domain experts around the world. A session was conducted at the premier international visualization conference, Viz ' 94 , by members of the project on this subject. Many researchers have expressed their views on the preferred method of implementation, but no one has implemented such a system on this large scale in terms of the number and diversity of components to be manipulated from within the VE.

Of the semiautomatic data analysis routines, only the depth-to-object has been implemented within the system. Several other methods have been or are undergoing analysis to verify their applicability. These routines and others already been identified for investigation and need to be incorporated into the system. These routines form an important part of the system. The current intent is not that these routines directly identify buried objects but rather provide indicators of where a human analysts need to focus their attention. This will help reduce the data overload placed on analysts and allow them to better 
comprehend the data they are working with and understand the processes and physical circumstances generating the sensor responses that produced the data.

For the reasons discussed above, the hardware system (HMD) was chosen for the current implementation. Future potential platforms include a wide variety of Unix and PC compatible architectures. The data base component currently runs on many Unix platforms and the data analysis modules run on almost all Unix machines. The data base and data analysis modules can be executed in a client/server environment across heterogeneous computers and operate as stand-alone. As the power of machines increase and their cost continues to decrease, a much larger class of machines will be potential platforms for this technology. All the vendors have indicated a willingness to reach a larger audience if the interest exists on the customer side and the vendor believes this interest and the platforms are robust enough to support their product.

\subsection{Depth-to-Object Algorithms}

In this section simple analysis methods for estimating buried object depths are investigated and discussed. The types of techniques discussed here are examples of the "Data analysis" component in Figure 5-4.

\subsubsection{Overview}

During review of the rapid prototype, geophysicists stressed the need for an analysis system that could support extraction of relationships in the geophysics survey data. It is recognized that such analysis for the digface data is a learning process and one must start with the basics and then gradually increase complexity. Working closely with the digface project personnel, the following analysis areas were of major interest:

- Depth-to-object estimation

- Contour analysis (determination of anomaly extrema)

- Anomaly characterization.

Magnetic anomaly extrema are one of the key factors that geophysicists look for in survey data. Typically, this is done by examining contour plots of the data and relating the contours to numeric values. This manual process is not necessarily difficult but is mundane, tedious, and error prone. Automated determination of extrema in a data set is theoretically straightforward, and we have developed software modules to effect extrema determination for the digface data sets. On gridded data sets, this process is straightforward. However, one cannot assume regular, gridded characterization data will always be available. Determination of extrema in irregular, unstructured data sets creates additional considerations such as evaluation of derivatives and survey edge effects, must be dealt with.

Magnetic anomalies can be used to obtain estimates of depth to the object causing the anomaly. For a given plane of data, the so-called "straight line" method (Josten and Roybal 1993; Vacquier et al. 1951) can be employed. This method is an empirical approach in which the length of a selected straight line on the anomaly surface is related to the sensor height and the shape factor and distance from the object causing the anomaly. 
If multiple planes of data are available, relatively simple approximations can be made to describe the expected power law behavior between the magnetic signature and distance from the object. When multiple levels of data are available, the fact that the measured magnetic influence of a buried object is expected to vary according to an inverse power law with distance from the object to the measuring device can be used. Several different schemes can be employed using two or more levels of data. The basic scheme consists of tracking anomaly extrema between multiple levels of data along with simplifying assumptions to allow estimation of the power law exponent. Application of this scheme to the digface data suggests that the exponent varies between two and five depending on the object and distance from the object. While this is a large variation, it appears that depth estimate bounds can be computed by using the range of exponents in the calculation. This approach was suggested and again the geophysicists' responses were that several reasonable estimates were considerably better than no estimates. Analysis is continuing to determine if improved methods based on multiple levels of data can be developed.

Application of the depth estimation methods are tedious for any significant amount of data. Software modules have been written to implement the straight line technique and have been tested using digface data and data from Josten and Roybal (1993). These modules are discussed below.

\subsubsection{Digface Data Analysis Software}

Initial development of software for automated analysis of data (primarily magnetic field and magnetic gradient data) collected from the digface project involved several subtasks. These subtasks included developing algorithms for gridding the data, identifying and locating peaks and associated valleys in the data for initial object identification, and developing an algorithm for depth-to-object estimates. To accomplish this task, three software packages were developed to run on a Macintosh computer (the algorithms are written in standard FORTRAN and are easily ported to other platforms). The first program (GRIDIT) reads the raw data ASCII files for each level and creates new ASCII files in which the data are properly "gridded," or the data is interpolated on a regular and uniform grid at each level. The second program (SEARCH) reads the data files created by GIIDIT and searches the data to locate all significant peaks in the data and the valleys (depressions) associated with these peaks. These significant peaks are assumed to result from buried objects. The locations of these peaks and valleys are then transferred to the module DEPTH that determines the depth of the buried object. The depth algorithm uses a straight slope technique suggested by Josten and Roybal (1993). A second depth estimation technique based upon a power law field attenuation was investigated. This technique has not been implemented in the depth algorithm but will be presented and discussed.

In the following sections, each of these three software packages is described in detail, including the analytical methodology used, the flow charts for the software, and test cases performed to verify accuracy and proper operation of the software. Results from the initial analysis of the data are presented, and recommendations for future work are made.

5.4.2.1. Program Description. Descriptions of each of the three main program modules are discussed below. 
5.4.2.1.1 Gridding Software (GRIDIT)-The GRIDIT program (composed in excess of 900 lines of FORTRAN code) reads the raw data files (stored as sets of X, Y, Z, Data in ASCII format) and rewrites these files with data in a uniform grid. Duplicate data points are removed and missing data are filled in. The program is based upon the two-dimensional bicubic interpolation methodology presented in Press et al. (1987). This technique interpolates within a data cell and the values of the raw data and the specified derivatives are reproduced exactly on the original grid points. A flow chart of the program is provided in Figure 5-6. Test cases were performed using linear and exponential data generated on a uniform grid as input. Hand calculations were performed to ensure that the derivatives and the interpolated data values were correct. No errors were found in the program results, and it was concluded that this algorithm performs as desired.

5.4.2.1.2 Peak-to-Valley Identification Software (SEARCH)-Before extensive automated analysis of the digface magnetic anomaly data can be accomplished, the locations of all of the significant peak-to-valley (P/V) pairs must be determined. This is the function of the SEARCH algorithm (some 1,600 lines of FORTRAN code). The data are obtained by reading the gridded data file generated by the GRIDIT program. Second order first derivatives of the data are calculated in both the $\mathrm{X}$ and $\mathrm{Y}$

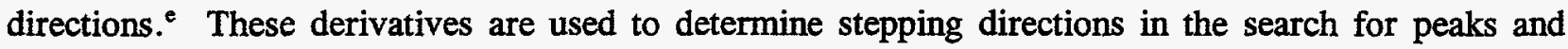
valleys. The data are searched to determine the maximum and minimum data values. It is assumed that these two values represent the most significant peak and valley (not necessarily associated). The location of the maximum value is used as a starting point to locate the valley associated with this peak. The search methodology, described next, is used in the program.

From a starting location ( $i, j)$, the location is stepped over 1 grid point, and the $X$ and $Y$ derivatives are used to determine a direction to step towards for the next search point. For example, in searching for a valley; if the $\operatorname{dD}(i, j) / d x$ value is negative, then the $X$ index $i$ is incremented by $1(i+1)$, if the $\mathrm{dD}(\mathrm{i}, \mathrm{j}) / \mathrm{dx}$ value is positive, then the $X$ index $\mathrm{i}$ is de-incremented by $1(\mathrm{i}-1)$. The same methodology is then used in the $\mathrm{Y}$ direction. This defines the location for the next search. The search continues and is terminated when the data value increases from the previous data value, indicating that the minimum occurred on the previous step. ${ }^{f}$ This methodology is repeated for a total of eight searches (four on the orthogonal directions and four on the $\mathbf{4 5}$ degree lines) starting from the same initial location. Usually only one or two unique local minimums are located using this technique. The smallest minimum is assumed to be the valley location associated with the peak.

After locating the valley associated with the maximum magnitude peak, the peak associated with the minimum magnitude valley. These two P/V pairs are located initially because they should be the most significant features of the data set, and it is desirable to ensure that they are properly located before attempting to locate all significant $\mathrm{P} / \mathrm{V}$ pairs.

e. Second order derivatives are used to help smooth local fluctuations in the derivatives.

f. This technique is not fool proof. The preference is to move along diagonals. It is possible to find a local minimum in one direction and have an adjacent data point actually be the minimum. Once the minimum has been identified using the derivatives, adjacent data points are checked to verify that it is truly a local minimum. Certain cases have been found in which the search continues along a X or Y line for some distance before the true local minimum is found. The software automatically performs this secondary search for the local minimum (or maximum if searching for a peak). 

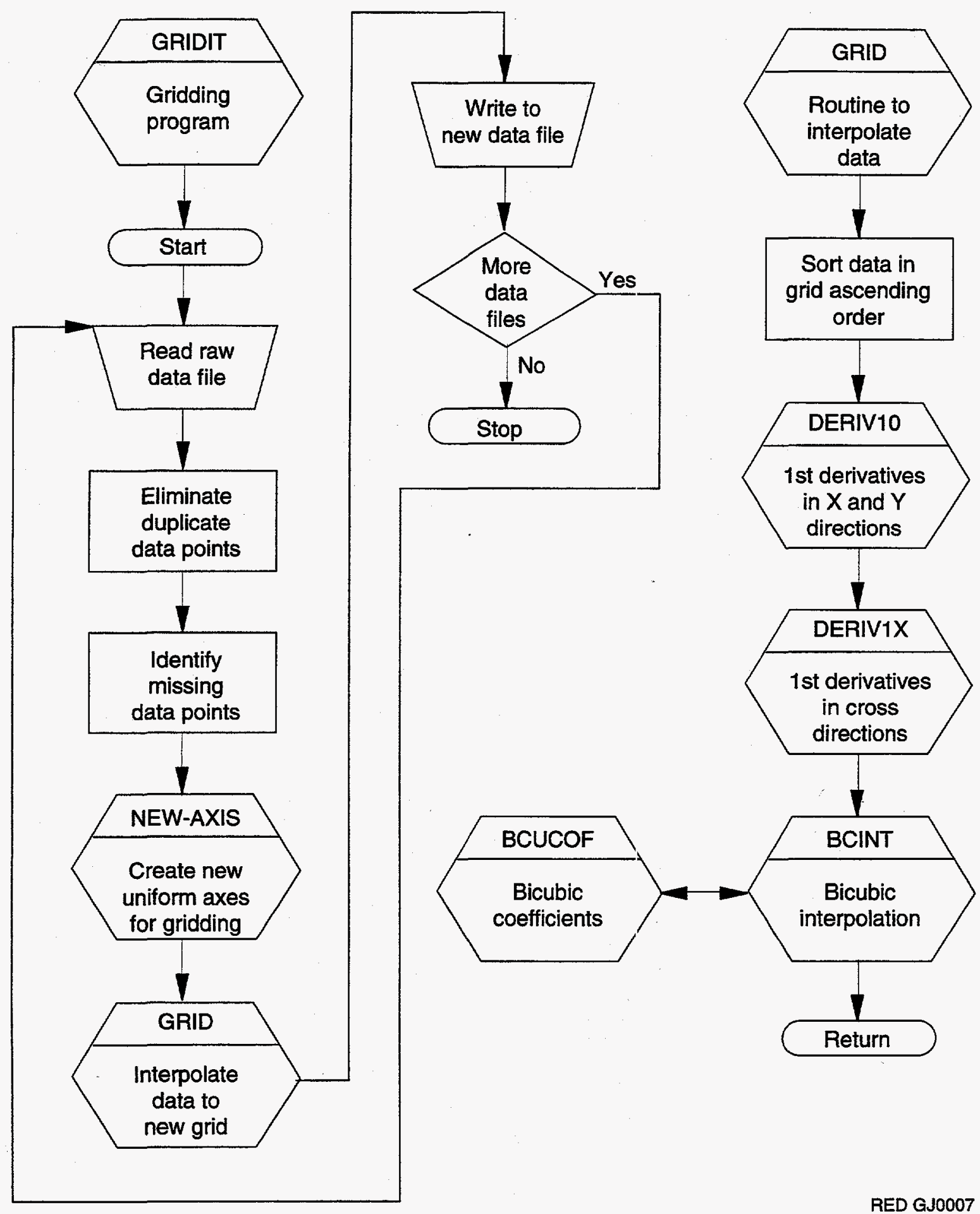

RED GJ0007

Figure 5-6. Flow chart for the GRIDIT program. 
The next step is to locate all local peaks in the data set. The same technique as previously presented is used (the reverse of the derivative signs is used for determining the search direction). The initial locations to start the searches are determined by dividing the grid area into equal increments in both the $\mathrm{X}$ and $\mathrm{Y}$ directions (a value of 10 increments is used). This results in peak locations, many of which are duplicates, and many of which may be insignificant peaks (such as a single data point blip in essentially level plane). ${ }^{g}$ These peak locations are sorted and duplicate locations eliminated. The remaining unique peak locations (which possibly still includes insignificant peaks) are then used as starting locations for searching for the valleys associated with each peak. After all the P/V pairs have been determined, insignificant $P / V$ pairs are eliminated based upon the magnitude of the data change from the $P / V$. If this magnitude is smaller than an arbitrary fraction of the difference between the entire data set maximum and minimum (a value of $7.5 \%$ is used), then the $\mathrm{P} / \mathrm{V}$ pair is considered to be insignificant and is discarded. The remaining P/V pairs are then available for further analysis, such as the estimation of object depth. Figure 5-7 shows a flow chart of SEARCH.

The search algorithm has been verified on several different data sets. The most extensive testing used the northern portion $(179<\mathrm{Y}<268$ ) of the Rapid Geophysical Surveyor (RGS) data set (Josten and Roybal 1993). The software identified a total of 13 unique significant P/V pairs, of which 6 are considered to be insignificant $P / V$ pairs (mainly because insignificant peaks were located). However, all significant $\mathrm{P} / \mathrm{V}$ pairs that had been previously manually located were correctly identified. Results from the search are presented in Table 5-1.

5.4.2. 1.3 Object Depth Estimation Software (DEPTH)-The depth estimation technique employed in the DEPTH algorithm (some 1,200 lines of FORTRAN code) is based on the technique suggested by Josten and Roybal (1993), in which "the horizontal extent of the steepest segment of a magnetic anomaly depends on the burial depth of the magnetic source." This results in a depth estimation given by

$$
d=\frac{s}{k}-h
$$

where

$$
\begin{aligned}
& \mathrm{d}=\text { estimated depth below ground surface } \\
& \mathbf{s}=\text { measured straight slope length } \\
& \mathbf{k}=\text { correction factor }(=0.55, \text { value suggested by Josten and Roybal 1993) } \\
& \mathbf{h}=\text { height of sensor above ground surface }(=1.0 \mathrm{ft} \text { for the RGS data set). }
\end{aligned}
$$

g. Another problem is a single point maximum on a boundary. The search routine will hit a boundary, and being constrained by the boundary, will locate a single point maximum along the boundary. These points are excluded because they are not true local maximums. 

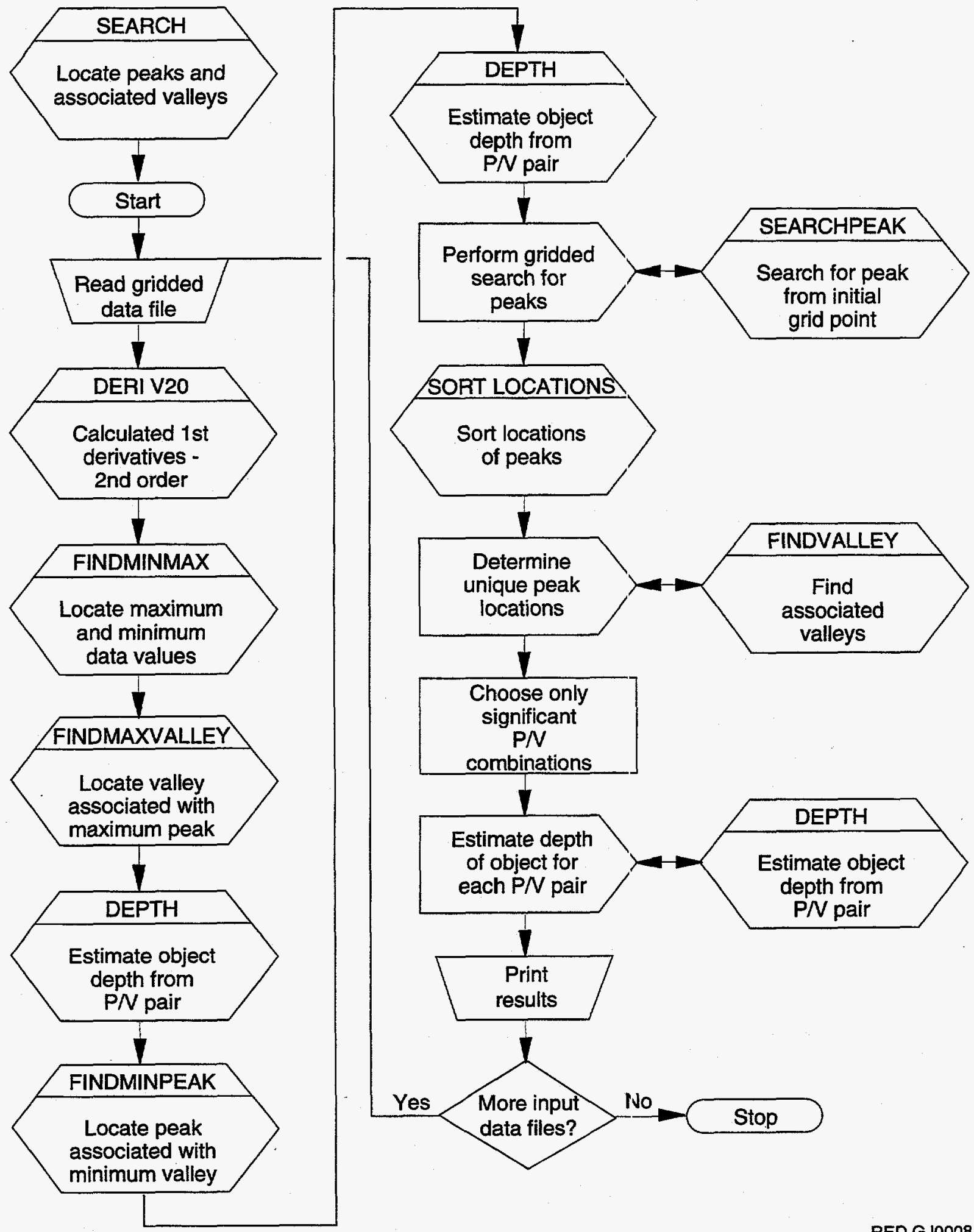

RED GJ0008

Figure 5-7. Flow chart for the SEARCH program. 
Table 5-1. Peak-to-valley locations determined by program SEARCH for northern RGS data.

\begin{tabular}{|c|c|c|c|c|c|c|}
\hline \multicolumn{3}{|c|}{ Peak locations } & \multicolumn{3}{|c|}{ Valley locations } & \multirow[b]{2}{*}{ Comments } \\
\hline $\begin{array}{c}\mathrm{X} \\
\text { (ft) }\end{array}$ & $\begin{array}{c}Y \\
\text { (ft) } \\
\end{array}$ & Gradient & $\begin{array}{c}\mathrm{X} \\
(\mathrm{ft})\end{array}$ & $\begin{array}{c}Y \\
\text { (ft) }\end{array}$ & Gradient & \\
\hline 46.0 & 183 & 1,140 & 37.0 & 180 & -190 & - \\
\hline 52.0 & 221 & 625 & 57.5 & 220 & -215 & - \\
\hline 52.5 & 187 & 1,020 & 56.5 & 189 & $-1,745$ & - \\
\hline 52.5 & 227 & 615 & 57.5 & 220 & -215 & - \\
\hline 54.5 & 227 & 505 & 57.5 & 220 & -215 & Insignificant peak \\
\hline 59.0 & 239 & 1,820 & 55.0 & 249 & $-1,625$ & - \\
\hline 62.5 & 229 & 355 & 58.0 & 220 & -215 & Insignificant peak \\
\hline 63.5 & 242 & 1,260 & 59.5 & 251 & $-1,440$ & Insignificant-hump on ridge \\
\hline 65.5 & 240 & 1,325 & 68.5 & 251 & $-1,380$ & Insignificant-hump on ridge \\
\hline 69.5 & 222 & 1,450 & 69.5 & 225 & $-1,040$ & - \\
\hline 69.5 & 240 & 1,055 & 68.5 & 251 & $-1,380$ & Insignificant peak \\
\hline 74.5 & 241 & 610 & 74.5 & 250 & -775 & Insignificant peak \\
\hline 89.0 & 227 & 4,270 & 91.0 & 233 & -370 & - \\
\hline
\end{tabular}

The major problem in using this technique is the automated determination of the horizontal straight slope length, s. The methodology finally selected and implemented in DEPTH is a two stage approach. The flow diagram is shown in Figure 5-8. The first stage involves a cubic polynomial fit to the subset of data between the data peak and valley. ${ }^{\mathrm{h}}$ The standard deviation (s) of the fit is used as a gage of how good the fit matches the data. A parameter (equal to $2 \%$ of the $P / V$ magnitude) is compared to the standard deviation; if the standard deviation is smaller than this parameter, then the fit is deemed acceptable and the process continues. If the standard deviation is larger than the parameter, then the cubic fit is repeated with one less data point. (The point nearest the valley is excluded first. A minimum of 6 points is required for the fit.) Once an acceptable fit is found, the first stage process continues. If an inflection point exists in the cubic fit within the bounds of the data subset range, a linear equation is calculated with the slope of the cubic equation at the inflection point that passes through the inflection point. The endpoints of the "straight slope" are set to be the locations where the difference between the cubic fit and the linear fit exceed some nominal value (a value of $1 \%$ of the P/V magnitude was used), working out from the inflection point in both directions.

h. This approach is performed five times, once in each of the $+X,-X,+Y$, and $-Y$ orthogonal directions, and once on the diagonal between the peak and valley. In the orthogonal directions, a local valley in the orthogonal direction is first found to define the data range for that particular fit. 


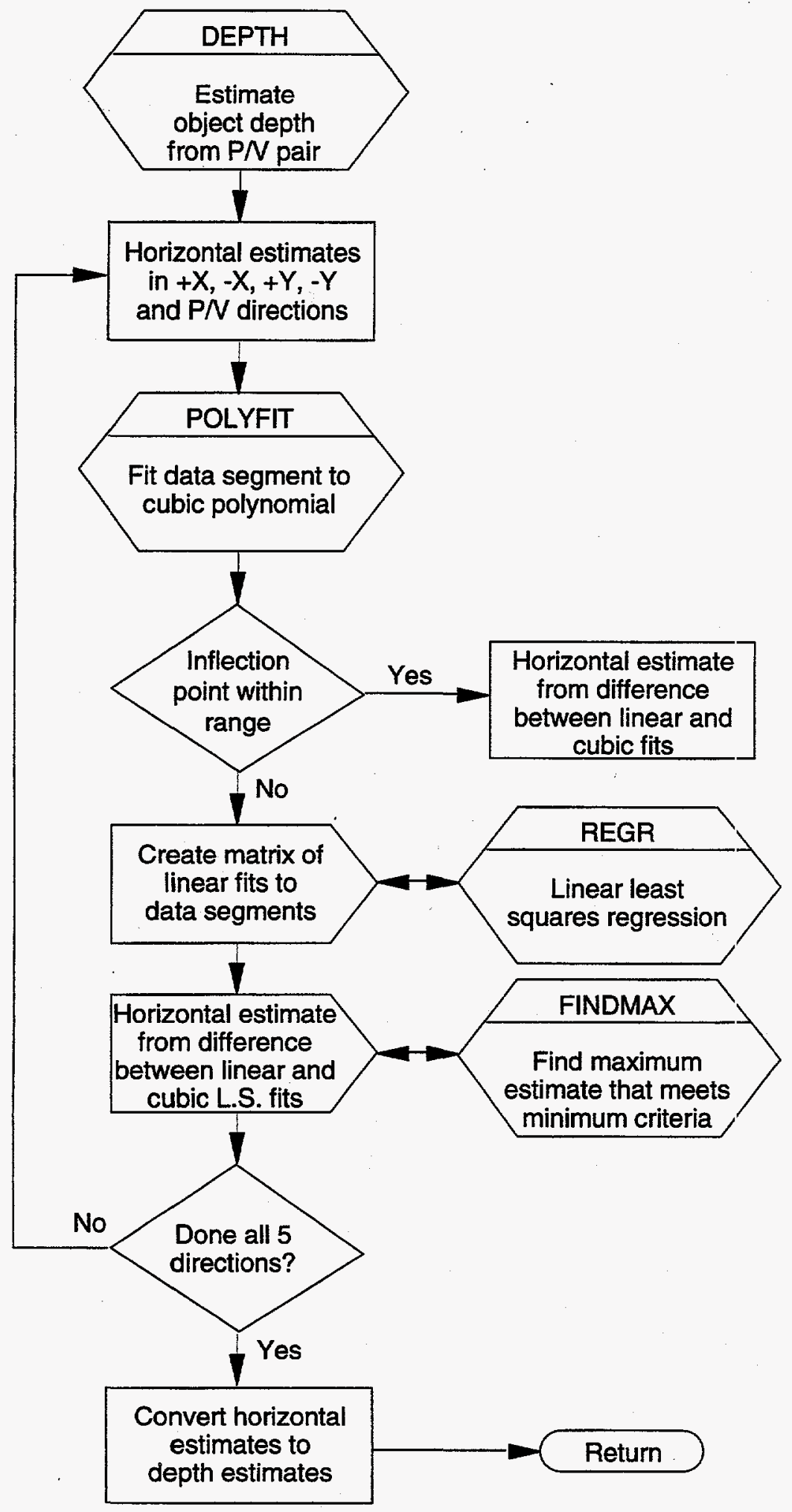

Figure 5-8. Flow chart for the DEPTH program. 
The second stage is used if an inflection point does not exist within the range of the data subset. In this case, a matrix of linear fits is generated for combinations of the data subset (down to the minimum allowable number of data points, currently set to 6). The locations at which the linear fit intersects the cubic fit are then determined, and the difference between the cubic and linear fits at the midpoint between the intersections is determined. If this difference is greater than the maximum allowable difference (as in the inflection analysis, the value of $1 \%$ of the P/V magnitude was used), then this fit is determined to be unacceptable and is flagged. Once all of the acceptable linear fits have been determined, the fit resulting in the maximum straight slope length, $s$, is chosen for determining the depth estimate, $d$.

This process is repeated five times, once in each of the orthogonal directions $(+X,-X,+Y$, and $-Y)$ and once along the line connecting the peak to the valley. These five estimated depths are then output to the screen and a file for further analysis.

5.4.2.2. Results. Results from the search algorithm are shown in Table 5-1, for the northern portion of the RGS data set $(179<\mathrm{Y}<268)$. The software identified a total of 13 unique $\mathrm{P} / \mathrm{V}$ pairs (that met the software significance criteria) of which 6 are considered to be insignificant $P / V$ pairs (mainly because insignificant peaks were located). However, all significant $\mathbf{P} / \mathrm{V}$ pairs that had been previously manually located were correctly identified.

Depth estimations for the RGS peak at $\mathrm{X}=89 \mathrm{ft}$ and $\mathrm{Y}=227 \mathrm{ft}$ [object F1/F2, the filing cabinets in the Characterization Cell (see Appendix A)] were obtained using the DEPTH software in the $+X,-X$, $+Y,-Y$, and $P / V$ directions. These results are provided in Table 5-2 and in Figures 5-9 through 5-13. In all cases, the inflection analysis technique was chosen, since the inflection point occurred within the data range. In Figure 5-9, the cubic fit chosen by the software (based upon the minimum allowable number of data points, 6) is compared to the linear fit from the inflection analysis and the cubic fit for the entire data subset. The estimated depth is about $50 \%$ of the actual depth, probably a result of the cubic fit resulting from the minimum number of data points, which failed to satisfy the minimum fit criteria. The fits and depth estimation in the $-X$ direction is shown in Figure 5-10. In this case, a total of 12 data points resulted in a cubic fit meeting the minimum fit criteria, and a depth estimation matching the actual object depth of $3.0 \mathrm{ft}$. The results for the $+\mathrm{Y}$ direction are shown in Figure 5-11. In this case, the cubic fit was again obtained from the minimum allowable number of data points, resulting in an depth estimation $60 \%$ of the actual depth. The results for the -Y direction are shown in Figure 5-12, where the cubic fit was again obtained from the minimum allowable number of data points, resulting in an depth estimation $117 \%$ of actual. The results for the line between the peak $(89,227)$ and the associated valley $(91,233)$ are shown in Figure $5-13$. There were a total of 7 data points interpolated between the peak and the valley, and the cubic fit through these 7 data points met the acceptance criteria. The inflection analysis resulted in a depth estimation of $3.1 \mathrm{ft}$ compared to the actual depth of $3.0 \mathrm{ft}$.

5.4.2.2.1 Uncertainties in Depth Estimation (Straight Slope Technique)-Object depth estimation using the straight slope technique was proposed by Josten and Roybal (1993) and the automated implementation was presented in the previous section (see footnote i). The implementation has been extended to include estimation of the uncertainties in the depth estimates calculated from the relationship given in Equation (5-1). A shape factor, $k$, of 0.37 is used based on the RGS digface data. 
Table 5-2. Results from depth estimation for RGS peak at X $=89 \mathrm{ft}$ and $\mathrm{Y}=227 \mathrm{ft}$ (object F1/F2).

\begin{tabular}{ccccc} 
Direction & $\begin{array}{c}\text { Estimated depth } \\
(\mathrm{ft})\end{array}$ & $\begin{array}{c}\text { Actual depth } \\
(\mathrm{ft})\end{array}$ & $\begin{array}{c}\text { Analysis type } \\
\text { Anic fit standard } \\
\text { deviation } \\
(\%)\end{array}$ \\
\hline$+\mathrm{X}$ & 1.6 & 3.0 & Inflection & 3.9 \\
$-\mathrm{X}$ & 3.0 & 3.0 & Inflection & 1.9 \\
$+\mathrm{Y}$ & 1.8 & 3.0 & Inflection & 3.2 \\
$-\mathrm{Y}$ & 3.5 & 3.0 & Inflection & 3.2 \\
$\mathrm{P} / \mathrm{V}$ & 3.1 & 3.0 & Inflection & 2.5
\end{tabular}

a. The standard deviation has been normalized by the magnitude of the $\mathrm{P} / \mathrm{V}$ data difference. The allowable criteria is $2 \%$. 


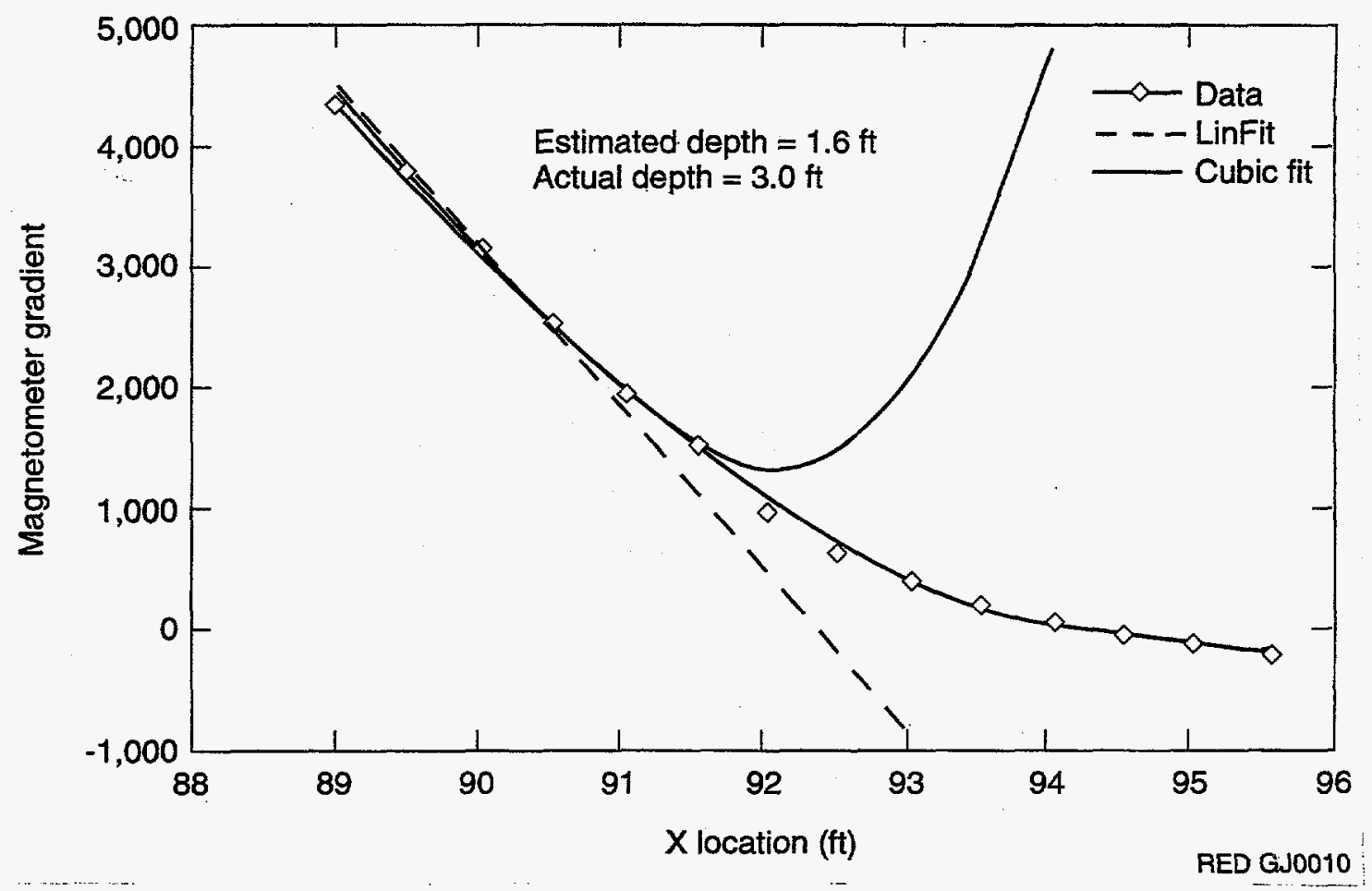

Figure 5-9. + X depth estimation results for RGS peak at $\mathrm{X}=89$ and $\mathrm{Y}=227 \mathrm{ft}$.

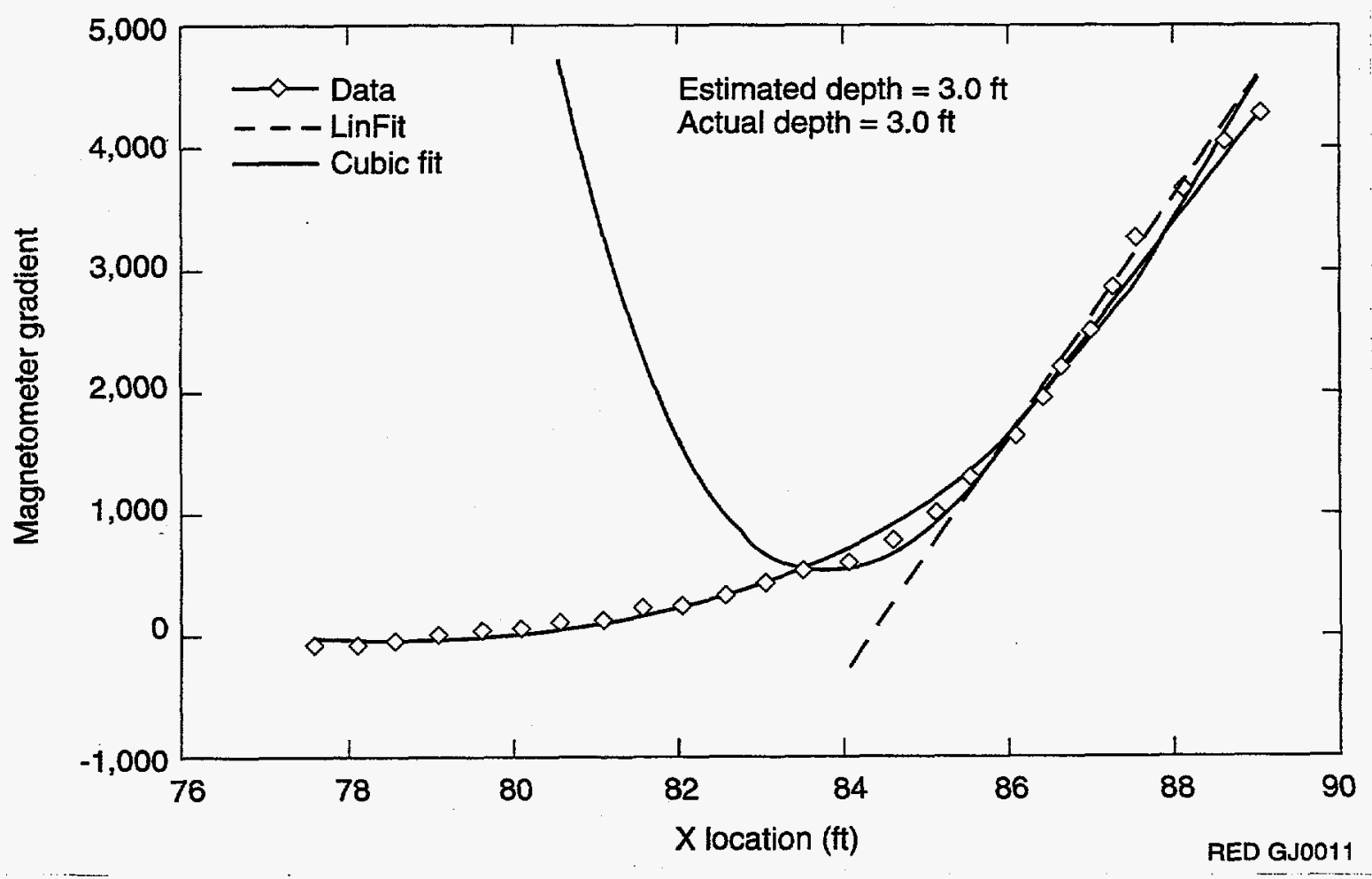

Figure 5-10. $-\mathrm{X}$ depth estimation results for RGS peak at $\mathrm{X}=89$ and $\mathrm{Y}=227 \mathrm{ft}$. 


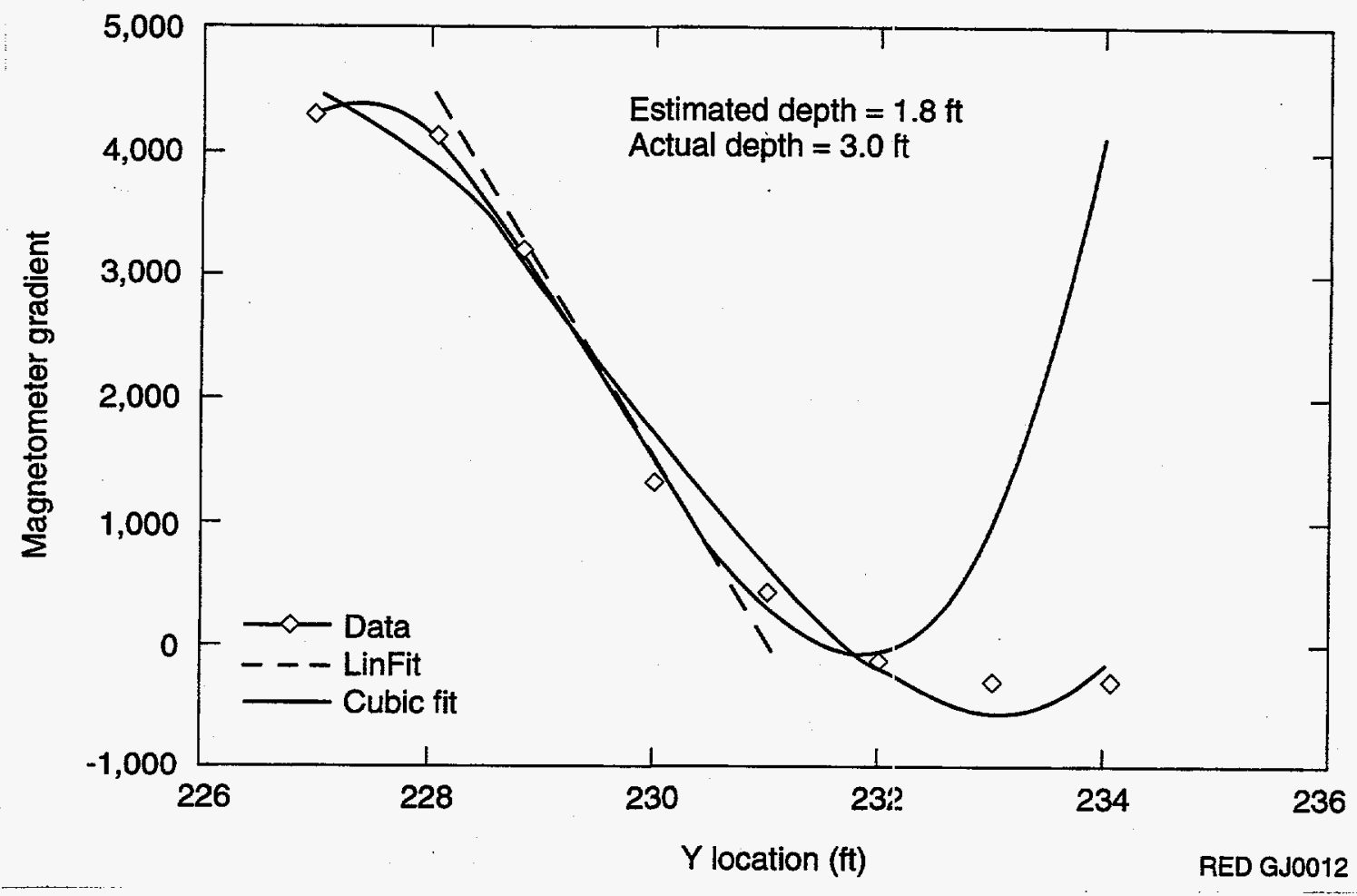

Figure 5-11. + $\mathrm{Y}$ depth estimation results for RGS peak at $\mathrm{X}=89$ and $\mathrm{Y}=227 \mathrm{ft}$.

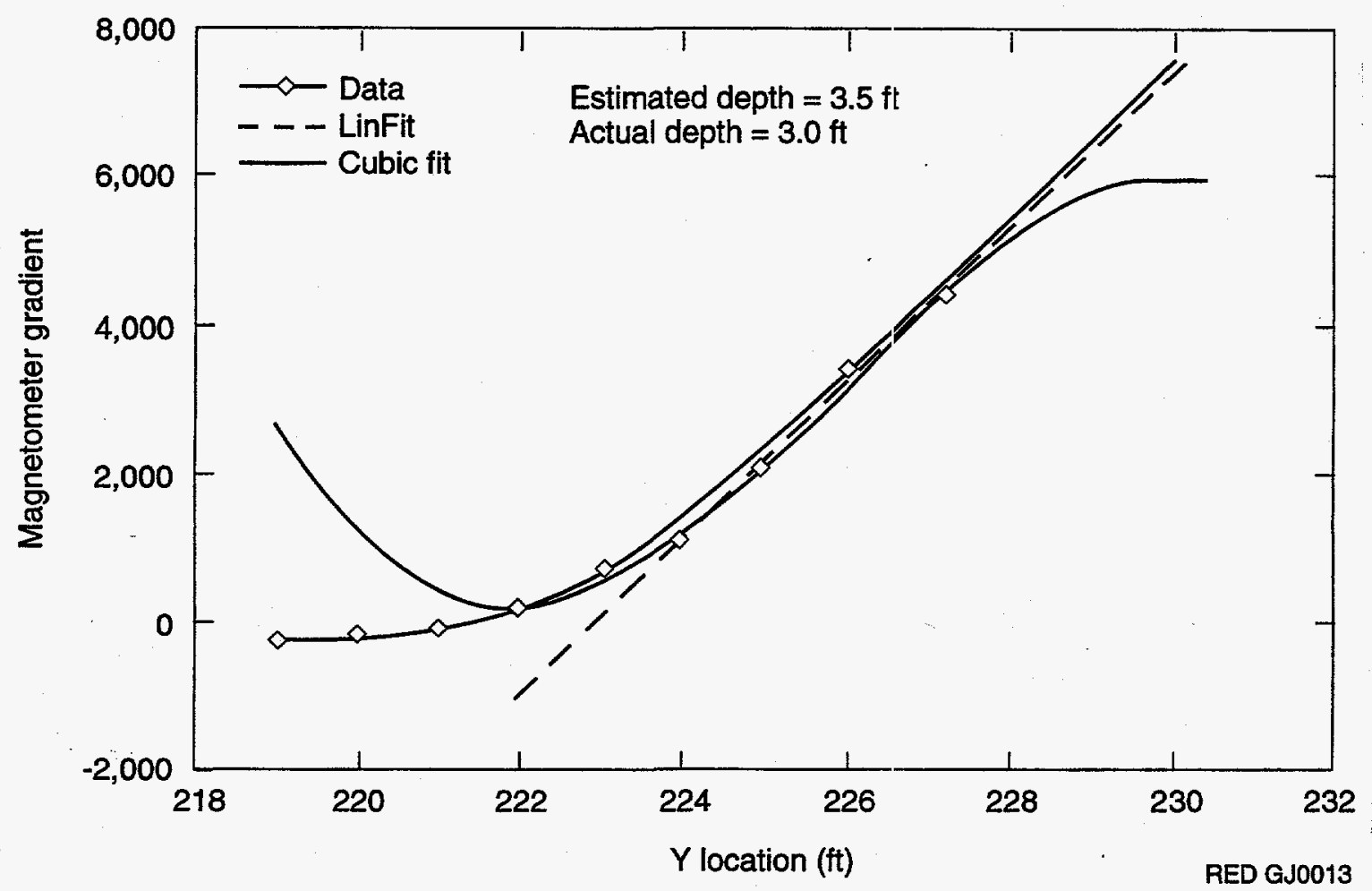

Figure 5-12. $-Y$ depth estimation results for $R G S$ peak at $X=39$ and $Y=227 \mathrm{ft}$. 


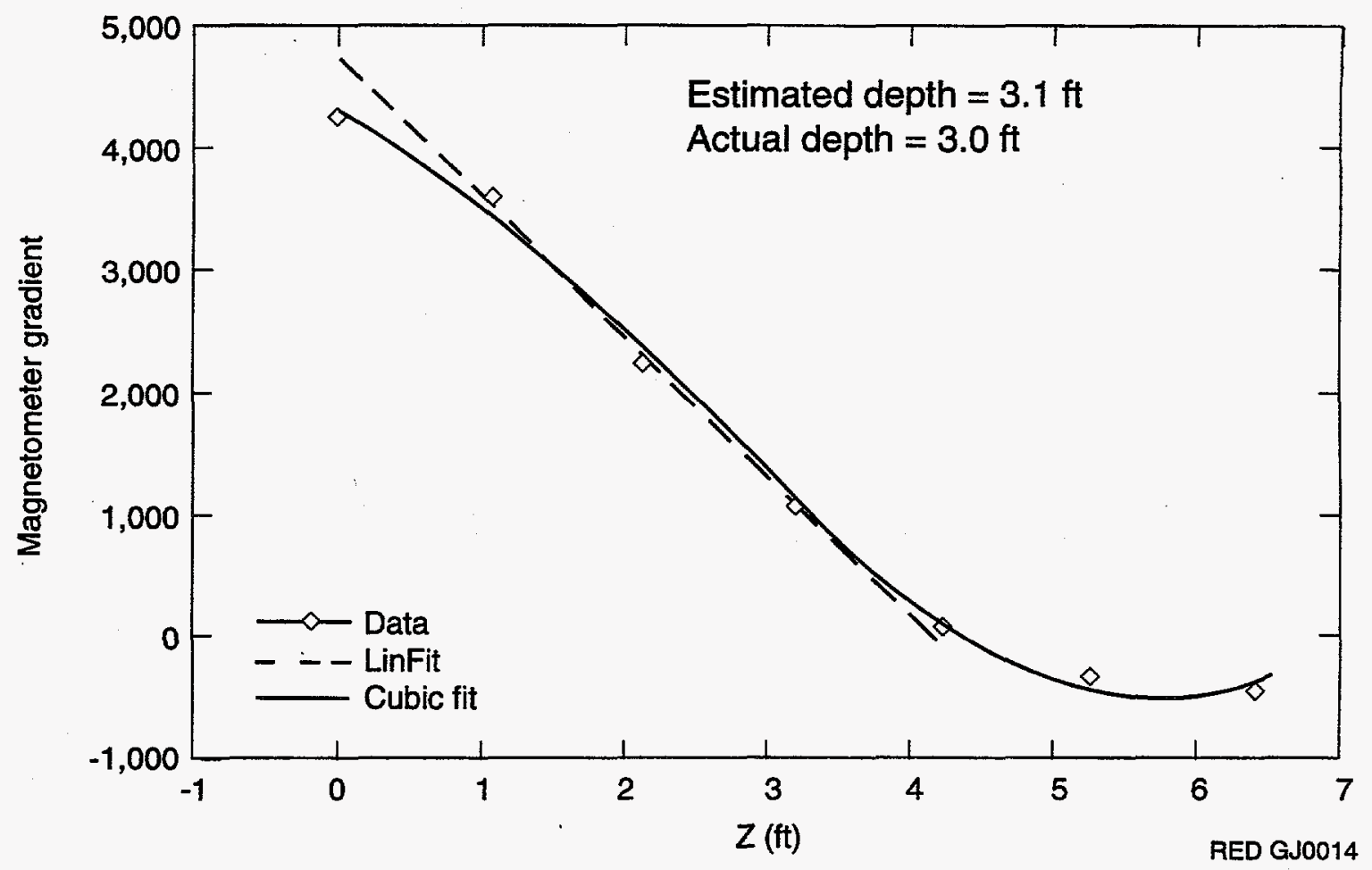

Figure 5-13. Peak-to-valley depth estimation results for $\mathrm{RGS}$ peak at $\mathrm{X}=89$ and $\mathrm{Y}=227 \mathrm{ft}$, valley at $\mathrm{X}=91$, and $\mathrm{Y}=233 \mathrm{ft}$.

Using the standard root-sum-square uncertainty analysis, the uncertainty in the depth estimation, $\Delta d$, can be obtained from Equation (5-1) as

$$
\begin{aligned}
\Delta d & =\left[\sum\left[\frac{\partial d}{\partial V_{i}} \Delta V_{i}\right]^{2}\right]^{1 / 2} \\
& =\left[\left[\frac{\dot{\Delta s}}{k}\right]^{2}+\left[\frac{s}{k^{2}} \Delta k\right]^{2}+(\Delta z)^{2}\right]^{1 / 2}
\end{aligned}
$$

where the symbols, $\Delta \mathrm{s}$, are the uncertainties in each variable (at the $95 \%$ confidence level), and $\mathrm{z}$ is the sensor-to-trolley distance.

A value of \pm 0.15 (based on geophysicists' input) is used for the uncertainty in the shape factor, $\Delta \mathrm{k}$. The uncertainty in the sensor-to-trolley distance, $\Delta \mathrm{z}$, is assumed to be zero (insignificant compared to the other uncertainty components). The uncertainty in the straight slope distance, $\Delta s$, is obtained from the uncertainties in the polynomial and linear least squares fits performed as part of the automated estimation technique. There are two techniques used, the first for the case in which the polynomial inflection point is within the data set range (the preferred method) and the second in which the inflection 
point analysis is not acceptable and the linear fits are obtained independently of the polynomial fit. The uncertainty for each of the coefficients of the polynomial fit (twice the standard deviation for a $95 \%$ confidence level) are first added to the fit coefficients, and the analysis repeated to obtain a second estimate of the depth. This value is subtracted from the original estimate is given a "high" side uncertainty estimate. This is repeated with the coefficient unce:tainties subtracted from the original polynomial coefficients to obtain the "low" side uncertainty estimate. The average of these two uncertainty estimates are then used as a symmetrical uncertainty estimate (in all trial cases examined, the high and low side estimates were close to being symmetrical). For the case in which the inflection analysis is not acceptable, the same type of uncertainty analysis is repeated, except only the coefficients of the linear fit are modified. ${ }^{i, j}$

This uncertainty analysis technique was applied to the digface experimental data set 2 (object 7 , at a buried depth of $5.78 \mathrm{ft}$ below the trolley) and presented in Table: 5-3. The analysis was performed in the four cardinal directions $(+X,-X,+Y,-Y$ ) and along the line connecting the $P / V$ of the magnetic anomaly (magnetic gradient data was used). The average of five estimated depths are compared to an average inversely weighted by the uncertainties as given by

$$
\bar{d}_{w t}=\frac{\sum_{i=1}^{N}\left[\frac{d}{\Delta d}\right]_{i}}{\sum_{i=1}^{N}\left[\frac{1}{\Delta d}\right]_{i}}
$$

The uncertainty in the weighted average is given by

$$
\Delta \bar{d}_{w t}=\left[\frac{1}{N} \sum_{i=1}^{N} \Delta d_{i}^{2}\right]^{1 / 2}
$$

The use of an average, inversely weighted by the individual uncertainties, minimizes the effects of individual depth estimates with large uncertainties and should provide a more reliable depth estimate for the object than an estimate obtained from any single randomly obtained line segment. Line segments chosen by an experienced analyst should provide a more reliable and accurate depth estimate that the line

i. J. L., Anderson, "Digface Data Analysis Software, Progress Report 5a," attachment to J. L. Anderson letter. to T. K. Larson, JLA-02-94, March 28, 1994.

j. It was found that modification of the coefficients for both the linear and polynomial fits resulted in failure of the routine to obtain a depth estimation. This is probably a direct result of applying the technique to the final solution rather than repeating the search and decision technique for the entire matrix, which would likely use a different data subset to determine the depth estimate. 
Table 5-3. Depth and uncertainty estimates for object 7, Experiment $2 .^{\mathrm{a}}$

\begin{tabular}{|c|c|c|c|c|c|c|c|c|c|c|c|c|c|c|c|c|}
\hline \multicolumn{3}{|c|}{ Peak location } & \multirow[b]{2}{*}{ Data } & \multicolumn{2}{|c|}{ (+X dir) } & \multicolumn{2}{|c|}{ (-X dir) } & \multicolumn{2}{|c|}{ (+Y dir) } & \multicolumn{2}{|c|}{ (-Y dir) } & \multicolumn{2}{|c|}{ (P/V dir) } & \multirow[b]{2}{*}{ Average } & \multirow{2}{*}{$\begin{array}{c}\mathrm{Wt} \\
\text { average }\end{array}$} & \multirow{2}{*}{$\begin{array}{c}\partial \mathrm{Wt} \\
\text { average }\end{array}$} \\
\hline $\mathbf{x}$ & $\mathbf{Y}$ & $\mathbf{Z}$ & & Depth & aDepth & Depth & $\partial$ Depth & Depth & $\partial$ Depth & Depth & JDepth & Depth & $\partial$ Depth & & & \\
\hline 12.00 & 8.5 & -0.58 & 1,789 & 0.00 & 0.00 & 5.65 & 2.06 & 5.10 & 1.83 & 5.97 & 2.18 & 5.11 & 1.83 & 5.45 & 5.43 & 1.98 \\
\hline 12.00 & 8.5 & -1.08 & 2,679 & 0.00 & 0.00 & 5.48 & 1.78 & 5.45 & 1.77 & 6.14 & 2.05 & 5.18 & 1.66 & 5.56 & 5.54 & 1.82 \\
\hline 12.00 & 8.5 & -1.58 & 4,094 & 0.00 & 0.00 & 5.52 & 1.60 & 5.53 & 1.60 & 6.24 & 1.89 & 5.24 & 1.48 & 5.63 & 5.60 & 1.65 \\
\hline 11.75 & 8.5 & -2.08 & 6,546 & 4.49 & 0.98 & 6.10 & 1.63 & 5.55 & 1.41 & 5.73 & 1.48 & 5.65 & 1.45 & 5.50 & 5.40 & 1.40 \\
\hline 11.75 & 8.5 & -2.58 & 10,898 & 4.97 & 0.97 & 5.50 & 1.18 & 5.68 & 1.26 & 5.73 & 1.28 & 5.39 & 1.14 & 5.46 & 5.43 & 1.17 \\
\hline 11.75 & 8.5 & -3.08 & 19,445 & 4.27 & 0.55 & 6.22 & 1.27 & 6.05 & 1.21 & 6.56 & 1.41 & 5.71 & 1.07 & 5.76 & 5.45 & 1.14 \\
\hline 11.75 & 8.5 & -3.58 & 38,597 & 4.92 & 0.54 & 5.85 & 0.92 & 5.44 & 0.75 & 6.43 & 1.16 & 7.11 & 1.43 & 5.95 & 5.70 & 1.01 \\
\hline 11.75 & 8.5 & -4.08 & 91,680 & 5.29 & 0.49 & 5.93 & 0.75 & 6.74 & 1.08 & 6.15 & 0.84 & 6.66 & 1.04 & 6.15 & 5.99 & 0.87 \\
\hline 11.75 & 8.5 & -4.58 & 231,868 & 5.63 & 0.43 & 5.64 & 0.43 & 7.49 & 1.18 & 0.00 & 0.00 & 7.18 & 1.05 & 6.48 & 6.10 & 0.85 \\
\hline
\end{tabular}

a. Object $7=$ horizontal 55 -gal drum (depth from trolley $=5.78 \mathrm{ft}$ )-Experiment 2 . Shape factor $=0.37$. 
segments systematically chosen by the software. The depth estimates (weighted averages) given in Table 5-3 are quite good, resulting in estimates within $\pm 6 \%$ of the correct value, which is well within the derived uncertainties. The algorithm tends to under estimate the depth of the object at large sensor-to-object distances and over estimate the object depth as the sensor scans get closer to the object. However, all depth estimates for this object are within 5 in. It should be noted that object 7 is a horizontal 55-gal drum that produces a good strong magnetic gradient signal and that the results for other objects may not be as good, particularly the wooden boxes for which the major source of the magnetic field is the metal strapping on the boxes. In addition, for these objıcts the value of the shape factor may be different.

5.4.2.3. Power Law Depth Estimation Technique. In addition to the straight slope depth estimation technique that has been discussed, a second technique bised upon the concept of a power law signal attenuation with increasing separation between the source (object) and sensor has been investigated.

Assuming that an inverse power relationship holds between the magnetic gradient measured at a specific level and the distance to the buried object (digface data show this to be true for isolated objects), an algorithm can be derived for estimation of the depth at which the object is buried. Assuming a relationship of the type,

$$
G_{i}=\frac{I}{d_{i}^{n}}
$$

.where

$\mathrm{G}_{\mathrm{i}}=$ the magnetic field gradient at level $\mathbf{i}$

I = some arbitrary intensity value resulting from the object

$\mathrm{d}_{\mathrm{i}}=$ the distance from the sensor to the buried object at level $\mathrm{i}$

$\mathrm{n}=$ the power relationship coefficient (expected, $2 \leq \mathrm{n} \leq 3$ ).

The distance from the sensor to the object is related to the clepth of the object, $d$, and the sensor head location, $z_{i}$, as

$$
d_{i}=d-\bar{z}_{i}
$$

The intensity value, I (assumed constant), can be eliminated by dividing Equation (5-5) at two different levels as

$$
\frac{G_{i}}{G_{i-1}}=\left(\frac{d_{i-1}}{d_{i}}\right)^{n}
$$


Equation (5-7) can be combined with Equation (5-6) and solved for the object depth as

$$
d=\frac{R z_{i=l}-z_{i}}{(1-R)}
$$

where

$$
R=\left[\frac{G_{i-1}}{G_{i}}\right]^{1 / n} .
$$

Equation (5-8) can be used in conjunction with the digface data from experiment zones 1,2, and 3 to evaluate this methodology for estimating the depth of objects. An initial evaluation is presented below in Table 5-4 based upon data from Experiment 2 for object 6, a box buried at a depth of $5.1 \mathrm{ft}$. The first two columns are object depth estimated for $n=2$ and $n=3$, and using a fixed reference for $\mathrm{i}-1$ of the initial level 0 (i.e., $i$ and $i=0$ ). The last two columns are object depth estimate for $n=2$ and $n=3$, using a sliding reference so that the current level and immediately previous level are used (i.e., $\mathrm{i}$ and $\mathrm{i}-1$ ). In all cases, the magnitude of the gradient is the change in magnitude between the peak and adjacent valley. For the upper levels ( 1 and 2), a coefficient of $n=3$ provides accurate prediction of the actual depth of $5.1 \mathrm{ft}$ for object 6 . However, the prediction progressively over predicts the object depth based upon data from the lower levels ( 3 to 8 ). The estimates based upon a $n=2$ significantly under predicts the depth at the upper levels and progressively improves the estimate as the sensor depth increases. More accurate predictions are obtained using the data from the initial level 0 as the basis for the analysis (e.g., the first two columns).

Further evaluation is presented in Table 5-5 for object 7, which was buried at a depth of $5.78 \mathrm{ft}$ (measured from the trolley). The results presented are for fits to Equation (5-8) for adjacent levels as the digface was lowered for both the peak magnetic gradient and the difference in the magnitudes of the magnetic gradient between the peak and associated valley. The power exponent, $n$, in Equation (5-8) can be observed to vary with the sensor-to-object separation. For the case using the P/V data, the exponent varies from -3.58 for levels 0 and 1 , to -2.46 for levels 7 and 8 , with an average value of -3.27 . For the case using the peak magnetic gradient data, the exponent varies from -4.00 for levels 0 and 1 , to -2.48 for levels 7 and 8 , with an average value of -3.44 . The average exponents are used to estimate the depths in Table 5-5 for both cases ( $\mathrm{P} / \mathrm{V}$ and peak). The depth estimates using the average exponents vary from underestimating the depth by $12 \%$ for the peak gradient at levels 0 and 1, overestimating the depth by $8 \%$ at levels 7 and 8 . The results for the $P / V$ gradient is similar, although the estimates are slightly better $(-8 \%$ and $6 \%)$.

Further evaluation is presented in Table 5-6 for object 11, which was a horizontal 55-gal drum buried at a depth of $5.58 \mathrm{ft}$ (measured from the trolley). These results are presented for fits to Equation (5-8) for adjacent levels as the digface was lowered, for both the peak magnetic gradient and difference in the magnitudes of the magnetic gradient between the peak and associated valley. The power exponent, $\mathrm{n}$, in Equation (5-8) can be observed to vary with the sensor to object separation. For the case 
Table 5-4. Power law depth analysis for object 6 (buried depth of $5.1 \mathrm{ft}$ ).

\begin{tabular}{|c|c|c|c|c|c|}
\hline \multirow[b]{3}{*}{$\begin{array}{c}\text { Level } \\
\text { number }\end{array}$} & \multirow[b]{3}{*}{$\begin{array}{l}\text { Level } \\
\text { (ft) }\end{array}$} & \multicolumn{2}{|c|}{ Depth of object } & \multicolumn{2}{|c|}{ Depth of object } \\
\hline & & $\mathrm{n}=3$ & $\mathrm{n}=2$ & $\mathrm{n}=3$ & $\mathrm{n}=2$ \\
\hline & & \multicolumn{2}{|c|}{$\begin{array}{c}\text { Shifting based on LO and } \\
\text { current level }\end{array}$} & \multicolumn{2}{|c|}{$\begin{array}{c}\text { Sliding based on previous and } \\
\text { current levels }\end{array}$} \\
\hline 0 & -0.58 & - & $=$ & - & - \\
\hline 1 & -1.08 & 5.08 & 3.66 & 5.08 & 3.66 \\
\hline 2 & -1.58 & 5.04 & 3.74 & 5.01 & 3.79 \\
\hline 3 & -2.11 & 5.21 & 3.97 & 5.46 & 4.26 \\
\hline 4 & -2.58 & 5.23 & 4.09 & 5.26 & 4.29 \\
\hline 5 & -3.08 & 5.31 & 4.27 & 5.46 & 4.59 \\
\hline 6 & -3.58 & 5.38 & 4.48 & 5.55 & 4.82 \\
\hline 7 & -4.08 & 5.47 & 4.71 & 5.64 & 5.05 \\
\hline 8 & -4.61 & 5.70 & 5.05 & 6.17 & 5.57 \\
\hline
\end{tabular}


Table 5-5. Power law depth analysis for object 7 (depth of $5.78 \mathrm{ft}$ ). ${ }^{\mathrm{a}}$

\begin{tabular}{ccccccccc}
\hline & & \multicolumn{3}{c}{ Peak location } & & \multicolumn{3}{c}{ Valley location } \\
\cline { 3 - 4 } \cline { 7 - 8 } Level & $\mathrm{Z}$ & $\mathrm{X}$ & $\mathrm{Y}$ & Data & & $\mathrm{X}$ & $\mathrm{Y}$ & Data \\
\hline 0 & -0.58 & 12.00 & 8.50 & 1,789 & & 10.75 & 14.00 & -737 \\
1 & -1.08 & 12.00 & 8.50 & 2,679 & & 10.50 & 13.50 & -949 \\
2 & -1.58 & 12.00 & 8.50 & 4,094 & & 10.25 & 13.00 & $-1,279$ \\
3 & -2.11 & 11.75 & 8.50 & 6,546 & & 10.50 & 12.50 & $-1,727$ \\
4 & -2.58 & 11.75 & 8.50 & 10,898 & & 10.25 & 12.00 & $-2,519$ \\
5 & -3.08 & 11.75 & 8.50 & 19,445 & & 11.00 & 12.00 & $-4,131$ \\
6 & -3.58 & 11.75 & 8.50 & 38,597 & & 10.75 & 11.50 & $-7,613$ \\
7 & -4.08 & 11.75 & 8.50 & 91,680 & & 11.00 & 11.50 & $-15,603$ \\
8 & -4.61 & 11.75 & 8.50 & 231,868 & & 11.00 & 11.00 & $-36,943$ \\
\hline
\end{tabular}

$\mathrm{S}$ to $\mathrm{O}$ estimates using $\mathrm{P} / \mathrm{V}$ ratio of adjacent levels $\left[\mathrm{G}(\mathbf{i})=1^{*} \mathrm{~d}(\mathbf{i})^{\wedge} \mathrm{N}\right]$

\begin{tabular}{|c|c|c|c|c|c|c|c|}
\hline \multicolumn{3}{|c|}{$\begin{array}{l}\text { of adjacent levels } \\
{\left[\mathrm{G}(\mathrm{i})=1^{*} \mathrm{~d}(\mathrm{i})^{\wedge} \mathrm{N}\right]} \\
\end{array}$} & \multicolumn{2}{|c|}{$\begin{array}{l}\text { Exponent values, } \mathrm{N} \\
\text { for adjacent levels }\end{array}$} & \multicolumn{3}{|c|}{$\begin{array}{l}\text { using peak ratio of adjacent levels } \\
\qquad\left[\mathrm{G}(\mathrm{i})=1^{*} \mathrm{~d}(\mathrm{i})^{\wedge} \mathrm{N}\right]\end{array}$} \\
\hline \multicolumn{3}{|c|}{$\mathbf{N}$} & \multirow[b]{2}{*}{$\mathrm{P} / \mathrm{V}$} & \multirow[b]{2}{*}{ Peak } & \multicolumn{3}{|c|}{$\mathbf{N}$} \\
\hline-3.00 & -3.27 & -4.00 & & & -3.00 & -3.44 & -4.00 \\
\hline 4.98 & 5.34 & 6.36 & -3.58 & -4.00 & 4.55 & 5.10 & 5.79 \\
\hline 5.16 & 5.50 & 6.43 & -3.49 & -3.77 & 4.87 & 5.40 & 6.05 \\
\hline 5.34 & 5.65 & 6.50 & -3.20 & -3.48 & 5.06 & 5.54 & 6.13 \\
\hline 5.44 & 5.71 & 6.47 & -3.53 & -3.72 & 5.28 & 5.71 & 6.26 \\
\hline 5.50 & 5.73 & 6.38 & -3.32 & -3.41 & 5.43 & 5.81 & 6.29 \\
\hline 5.57 & 5.77 & 6.31 & -3.29 & -3.35 & 5.53 & 5.85 & 6.25 \\
\hline 5.62 & 5.78 & 6.21 & -3.27 & -3.36 & 5.58 & 5.83 & 6.15 \\
\hline 6.01 & 6.15 & 6.55 & -2.46 & -2.48 & 5.99 & 6.23 & 6.53 \\
\hline $\begin{array}{l}\text { Mag } \\
\text { Dept } \\
\text { Dept }\end{array}$ & $\begin{array}{l}\text { adient } \\
\text { sensor } \\
\text { trolley }\end{array}$ & $\begin{array}{l}\text { ject } 7 \text {. } \\
5.20\end{array}$ & & & & & \\
\hline
\end{tabular}

Depth estimates (from trolley) using peak ratio of adjacent levels $\left[\mathrm{G}(\mathrm{i})=1 * \mathrm{~d}(\mathrm{i})^{\wedge} \mathrm{N}\right]$
Exponent values, $\mathbf{N}$ for adjacent levels 
Table 5-6. Power law depth analysis for object 11 (depth of $5.58 \mathrm{ft}$ ).

\begin{tabular}{ccccccccc}
\hline & & \multicolumn{3}{c}{ Peak location } & & \multicolumn{3}{c}{ Valley location } \\
\cline { 3 - 5 } Level & $\mathrm{Z}$ & $\mathrm{X}$ & $\mathrm{Y}$ & Data & & $\mathrm{X}$ & $\mathrm{Y}$ & Data \\
\hline 0 & -0.58 & 13.00 & 6.50 & 2,281 & & 13.00 & 11.00 & -855 \\
1 & -1.08 & 13.00 & 6.50 & 3,058 & & 13.00 & 11.00 & $-1,384$ \\
2 & -1.58 & 13.00 & 7.00 & 4,119 & & 13.00 & 10.50 & $-2,361$ \\
3 & -2.11 & 13.00 & 6.50 & 5,552 & & 13.00 & 10.50 & $-3,761$ \\
4 & -2.58 & 13.00 & 7.00 & 8,383 & & 13.00 & 10.00 & $-5,696$ \\
5 & -3.08 & 13.00 & 7.00 & 12,650 & & 13.00 & 10.00 & $-9,688$ \\
6 & -3.58 & 13.00 & 7.00 & 18,830 & & 13.00 & 10.00 & $-15,290$ \\
7 & -4.08 & 13.00 & 7.00 & 22,880 & & 13.00 & 10.00 & $-30,500$ \\
\hline
\end{tabular}

$S$ to $O$ estimates using $P / V$ ratio of adjacent levels $\left[\mathrm{G}(\mathrm{i})=\mathrm{I}^{*} \mathrm{~d}(\mathrm{i})^{\wedge} \mathrm{N}\right]$
Exponent values, $\mathrm{N}$ for adjacent levels

$S$ to $O$ estimates using peak ratio of adjacent levels $\left[\mathrm{G}(\mathrm{i})=\mathrm{I}^{*} \mathrm{~d}(\mathrm{i})^{\wedge} \mathrm{N}\right]$

\begin{tabular}{ccccccccc}
\hline & $\mathrm{N}$ & & & & & \multicolumn{3}{c}{$\mathrm{N}$} \\
\cline { 8 - 10 }-2.00 & -2.56 & -3.00 & & $\mathrm{P} / \mathrm{V}$ & Peak & -2.00 & -2.14 & -3.00 \\
\hline 3.71 & 4.51 & 5.14 & -3.30 & -2.78 & 4.25 & 4.48 & 5.95 \\
3.99 & 4.72 & 5.31 & -3.21 & -2.53 & 4.69 & 4.92 & 6.37 \\
4.62 & 5.39 & 6.00 & -2.55 & -2.10 & 5.22 & 5.44 & 6.89 \\
4.76 & 5.43 & 5.97 & -2.84 & -2.83 & 4.77 & 4.93 & 5.98 \\
5.01 & 5.61 & 6.09 & -2.53 & -2.26 & 5.27 & 5.43 & 6.48 \\
5.70 & 6.35 & 6.88 & -1.90 & -1.78 & 5.85 & 6.02 & 7.11 \\
6.07 & 6.69 & 7.19 & -1.56 & -0.68 & 8.97 & 9.32 & 11.53
\end{tabular}

a. Magnetic gradient data-object 11 (horizontal 55-gal drum).

Depth from sensor head $=5.00$.

Depth from trolley $=5.58$. 
using the P/V data, the exponent varies from -3.30 for levels 0 and 1 , to -1.56 for levels 7 and 8 , with an average value of -2.56 . For the case using the peak magnetic gradient data, the exponent varies from -2.78 for levels 0 and 1 , to -0.68 for levels 7 and 8 , with an average value of -2.14 . The average exponents are used to estimate the depths in Table 5-6 for both cases ( $P / \mathrm{V}$ and peak). The depth estimates using the average exponents vary from underestimating the depth by $19 \%$ for the peak gradient at levels 0 and 1 , overestimating the depth by $67 \%$ at levels 7 and 8 . The results for the P/V gradient are similar, although the estimates are slightly better (-19\% and $20 \%)$.

Evaluation of the power law depth estimation technique for another horizontal 55-gal drum is presented in Table 5-7 for object 17, which was buried at a depth of $4.48 \mathrm{ft}$ (measured from the trolley). The power exponent, $n$, in Equation (5-8) can be observed to vary with the sensor-to-object separation. For the case using the P/V data, the exponent varies from -4.25 for levels 2 and 3 , to -3.08 for levels 4 and 5 , with an average value of -3.56 . For the case using the peak magnetic gradient data, the exponent varies from -5.34 for levels 2 and 3, to -3.33 for levels 4 and 5 , with an average value of -4.37 . The average exponents are used to estimate the depths in Table 5-7 for both cases ( $\mathrm{P} / \mathrm{V}$ and peak). The depth estimates using the average exponents vary from underestimating the depth by $13 \%$ for the peak gradient at levels 2 and 3, to overestimating the depth by $11 \%$ at levels 4 and 5 . The results for the P/V gradient is similar, although the estimates are slightly better $(-12 \%$ and $9 \%)$.

It appears that the depth estimation technique based upon the power law field attenuation has potential merits and should be investigated further. It further appears that using the difference between the P/V magnetic gradients (e.g., the anomaly range) provides nominally more accurate and consistent results, although the range of values for the power exponent is fairly large. In general, the values of the exponents decrease with decreasing sensor to object separation. It may be possible to model this trend in the exponent to obtain more accurate results. It is further observed that the results for object 11 are significantly different than for objects 7 and 17, even though all three objects are horizontal 55-gal drums. No explanation for this discrepancy is offered at this time.

5.4.2.4. Conclusions and Recommendations for Future Work. The software to perform the data gridding, $\mathrm{P} / \mathrm{V}$ search and identifications, and depth estimation based upon the horizontal straight slope technique have been successfully developed and evaluated. The gridding software performed as required. The $\mathrm{P} / \mathrm{V}$ search software successfully identifies all manually identified $\mathrm{P} / \mathrm{V}$ combinations for the northern RGS data set, although several insignificant P/V combinations were also located. This software needs extensive verification by running on numerous other data sets. The depth estimation algorithm provides reasonable estimates of the object depth, although with a wide range of estimates. The power attenuation technique applied to the magnetic gradient should be further investigated, formalized, and included in the depth estimation algorithm.

The depth estimation algorithm was applied in various directions from anomaly peaks to provide multiple estimates of depth. As expected, the multiple depth estimates showed variation due to noise and interference effects. In spite of this, geophysicists examining the results were pleased and indicated that several reasonable estimates were much better than no estimate at all. They requested the ability to apply the straight line technique in an interactive, analyst selected manner. Analysts presently apply the technique by hand on a single line profile. They desired an automated technique with the ability to provide the algorithm a line segment(s) defining points in three-space on the data surface to operate the algorithm over. In this manner, as many (or few) estimates as desired by the analyst could be generated 
Table 5-7. Power law depth analysis for object 17 (depth of $4.48 \mathrm{ft}$ ). ${ }^{\mathrm{a}}$

\begin{tabular}{ccccccccc}
\hline & & \multicolumn{3}{c}{ Peak location } & & \multicolumn{3}{c}{ Valley location } \\
\cline { 3 - 5 } Level $^{\mathrm{b}}$ & $\mathrm{Z}$ & $\mathrm{X}$ & $\mathrm{Y}$ & Data & & $\mathrm{X}$ & $\mathrm{Y}$ & Data \\
\hline 0 & -0.58 & 7.25 & 6.00 & 1,249 & & 3.75 & 3.50 & $-1,817$ \\
1 & -1.08 & 7.25 & 6.00 & 2,333 & & 4.25 & 3.50 & $-2,417$ \\
2 & -1.58 & 7.25 & 6.00 & 4,797 & & 4.50 & 3.50 & $-3,621$ \\
3 & -2.11 & 7.25 & 6.00 & 14,100 & & 5.00 & 3.50 & $-5,751$ \\
4 & -2.58 & 7.25 & 6.00 & 34,780 & & 5.50 & 3.50 & $-9,956$ \\
5 & -3.08 & 7.25 & 6.00 & 96,200 & & 5.75 & 3.50 & $-18,400$ \\
\hline
\end{tabular}

$S$ to $O$ estimates using $P / V$ ratio of adjacent levels $\left[\mathrm{G}(\mathrm{i})=\mathrm{l}^{*} \mathrm{~d}(\mathbf{i})^{\wedge} \mathrm{N}\right]$
Exponent values, $\mathbf{N}$ for adjacent levels
$S$ to $O$ estimates using peak ratio of adjacent levels $\left[\mathrm{G}(\mathrm{i})=1 * \mathrm{~d}(\mathrm{i})^{\wedge} \mathrm{N}\right]$

\begin{tabular}{lllllllll}
\hline & $\mathrm{N}$ & & & & & \multicolumn{3}{c}{$\mathrm{N}$} \\
\cline { 8 - 10 }-3.00 & -3.56 & -4.00 & & $\mathrm{P} / \mathrm{V}$ & $\mathrm{Peak}$ & -4.00 & -4.37 & -5.00 \\
\hline 4.26 & 4.90 & 5.40 & -3.19 & -4.55 & 4.04 & 4.33 & 4.84 \\
3.96 & 4.45 & 4.83 & -3.60 & -4.53 & 4.11 & 4.37 & 4.80 \\
3.62 & 3.94 & 4.20 & -4.25 & -5.34 & 3.73 & 3.910 & 4.19 \\
4.19 & 4.53 & 4.80 & -3.68 & -4.08 & 4.55 & 4.76 & 5.11 \\
4.44 & 4.73 & 4.97 & -3.08 & -3.33 & 4.81 & 4.99 & 5.30
\end{tabular}

a. Magnetic gradient data-object 17 (horizontal 55-gal drum).

Depth from sensor head $=3.90$.

Depth from trolley $=4.48$.

b. No peak at levels 0 and 1 . 
rapidly. Human expert interpretation would then determine which of the estimates are most appropriate. Work is directed toward providing this interactive capability.

Geophysics personnel suggested other avenues of potentially useful analysis. Anomaly characterization using contour area analysis was suggested. The question was posed as to whether or not the shape of an anomaly correlated to the characteristics of the object causing the anomaly. One manner of addressing this question is to relate the contour area at some percentage of the anomaly range to the contour area at some other value of the range. Preliminary manual evaluations show some promise, but additional data are needed to fully explore this concept. Automation of the process will be required to fully exploit the idea since manual implementation is extremely tedious. It is recommended that this work be conducted to determine the applicability of contour area analysis for waste classification.

\subsection{Automatic Target Recognition Evaluation}

Sections 5.5 and 5.6 describe investigations of two inference techniques for potential application to interpretation of characterization data. The potential role of these techniques in the overall system is shown in Figures 4-1 and 5-4. Results from inference methods could be provided back to the VWAE for user information and interaction.

There are important similarities between the two inference methods: ATR and neural networks. Both techniques use the concept of training and are potentially able to learn from experience and improve capabilities as more experience in data interpretation is gained. Both are instances of pattern recognition techniques and can be applied to sensor data from multiple sensors measuring distinct and possibly unrelated physical properties. The ability to learn from and apply information from multiple sources represents a potentially powerful method of "data fusion." At the same time, since both techniques apply mathematical methods for pattern recognition, there is loss of "traceability" of how, in a deterministic sense, the resulting answer was obtained. Care is required in how the result information is presented and applied in the overall system. The present feasibility studies assume that the training and subsequent use of information will be under the supervision of scientists familiar with the techniques. In the digface application, the inference information is intended to be used in conjunction with other interpretation techniques, including direct inspection of unprocessed sensor data.

The use of training data in both techniques, although straightforward conceptually, can be somewhat of an art in practice. Training provides the technique with examples of sensor response for known targets. The system adjusts itself so that there is correlation established between sensor data and the target. Once trained, the system can be applied to new situations in which new data are scanned and potential targets identified. In practice, care must be taken in training. If ambiguous, unrepresentative, or inadequate training sets are used, the technique may not learn enough to be able to detect targets as intended. On the other hand, the possibility of overtraining also exists in which the technique may be taught to recognize characteristics specific to the training set that are not general target characteristics. In the case of neural networks, researchers have studied the optimum training set size required for good generalization (Baum and Haussler 1989; Mehrota et al. 1991; Soulie et al. 1987).

Although similar in nature of use, there are several important distinctions between the ATR and neural network approaches. The ATR approach uses a deterministic mathematical technique during the training process to produce specific coefficients resulting from its error minimization methodology. In contrast, the neural networks are less deterministic. The training process results in a network where the 
"learning" is spread out and inherent in the weighing factors distributed throughout the processing elements (PEs) of the net. The learning method and application of knowledge to new situations can only be followed at a conceptual level, the details are unavailable for human understanding or review.

In the initial feasibility studies that evaluated the two techniques, different assessment methods were used. For the ATR evaluation, the system was trained to classify scenes based on three categories: barrel targets, box targets, and background. The data used for training could come from different experiments in which, for training, the operator identified for the system areas that had the features of interest, (i.e., barrel, box, or background). For this study, the information used in training for a given sensor in a given experiment came only from one level. (The system was not trained to use any information on how a given sensor responds as the distance to the target varies.) Once trained, the ATR system was tested on how successful it was in identifying targets (e.g., barrels, boxes, background) in scenes different from the ones used in training. This approach conforms to the expected application of the ATR system for assisting in digface data interpretation. At the same time, as discussed later, training the system to classify scenes into the three categories, barrel, box, or background, may have been unnecessarily challenging. At first, the most important distinction to make is whether a region represents target (whether barrel or box) or background. Nevertheless, as discussed below, the system performed rather well in the (more challenging) mode it was tested in.

In the case of neural networks (Section 5.6), a slightly different approach was used in the study of feasibility for the data interpretation application. The initial step was to train the net to distinguish target from background for each of the sensor measurements. Because of the limited data sets available for both training and testing, we decided to start off by training the networks by using files that included only every third point from the raw data files. This subsampling was done to produce the minimum training file while leaving some data available for testing the network. Testing under these situations allowed initial evaluation of appropriate methods (e.g., backpropagation versus other; number of hidden layers, threshold functions). For the neural network evaluation, the classification used was only into target or background. Unlike the ATR study, no attempt was made to distinguish between barrel targets and box targets. After the initial net evaluation task was completed, subsequent tasks evaluated performance using data from different combinations of sensors. This provides insight on what information is really used by the network as a basis for classifying targets. Next, information from different levels of each experiment for each sensor and for combined sensors was used in training and testing. (This type of evaluation was not performed for the ATR study.) Finally, different combinations were tried where networks trained on certain data were subsequently evaluated for ability to distinguish targets in new scenes not previously encountered (i.e., in the training set). As mentioned above, the type of mode expected will be the one used for the digface data interpretation application.

\subsubsection{Automatic Target Recognition System Overview}

An ATR system was evaluated for use in assisting in data interpretation of sensor data obtained during the digface characterization process. The potential role of a target recognition system is to aid the operator or geophysicist. The ATR system could be used to automatically scan incoming data and highlight areas of potential targets of interest. As described below, these targets would be defined by expert users during a training process. The target recognition system, if successful, could flag areas of potential targets that would then be verified by a human expert. This role for the system would support 
human experts by allowing them to focus attention specifically on the areas of suspected targets in making characterization determinations.

The ATR system was originally developed for the DOE Office of Arms Control to aid in identifying targets, principally military ones, from sensor data collected by an airborne reconnaissance system (Partin et al. 1994). The system essentially finds targets in digitized images. In use, the system is presented with one or more images of a scene, and the system identifies the presence and location of targets. The system was designed primarily for fast screening of large amounts of data to select those scenes for human inspection that might contain targets of interest.

The term target has a general meaning for this system. The targets can be military targets such as airplanes or trucks. However, a target in the general sense is anything that is potentially recognizable in the image data. Similarly, the term image has a general meaning. Image data can include any array of digital data values versus position in two dimensions. A standardized digitized video picture, with intensity versus $x$ and $y$ coordinates, is such an image. However, instead of intensity, the image data for the system could be any quantity, with the only requirement that the data be in the form of a two-dimensional array of values.

Since the system is designed to simultaneously process images comprising several different types of data for the same scene, it is essentially an image data fusion system. The presumption is that a target can be recognized more reliably if there are several different types of image data instead of just one. However, the system is fully functional with as few as one image per scene.

Given such functionality, we judged the ATR system to have potential use for interpreting sensor data obtained during scans at the digface. A single scan would result in data obtained over two dimensions for a number of different sensors. These separate images could then be processed by the ATR system to flag regions containing targets of interest that the system was previously trained to recognize.

\subsubsection{System Description}

\section{Hardware}

This ATR system comprises, as a minimum, a PC with a standard monitor and keyboard. The main computer chassis contains certain special hardware, including some transputers (parallel processors) and a high-capacity hard disk. An additional, high-resolution video display unit for viewing images is recommended but not essential. A printer, preferably a color printer, can also be added to the system. The prototype system currently in use could be replaced for less than $\$ 30,000$.

\section{Software}

This ATR system uses basic, standard software such as the DOS-operating system normally supplied with the PC and the host-transputer interface software normally supplied with the transputer network. Other than this, all the software was developed by the INEL for DOE. 


\subsubsection{Process Description}

To use the system a training process is first applied. A knowledgeable person defines the targets of interest and background scenes in a number of training examples. Once the system has been trained, it can be applied to find targets in new situations.

This ATR system is based on the assumption that targets can be distinguished from background by a quantitative measurement of the presence of local features. The term local normally implies that the features are small enough to be defined within a region that is small compared to the size of a target, although the formalism does not impose any limit on the size of the features (except that the features must be smaller than the whole image). Examples of simple features are edges, lines, intensity averaged over a small region, and speckle intensity. More complicated features include run length other descriptors of size or shape of some region, and local co-occurrence matrices. As a local feature, one could define something as complicated as a detailed image of a particular target object, but this would not be a normal or easy use of the present formalism and would not be covered by the usual interpretation of the phrase local feature. For each feature, we must be able to calculate a value at each pixel in the scene. For example, for a lines feature, at a given pixel, we might use as the quantitative value the brightness of a line passing through that pixel in one of the scene images. The value would be more positive for a brighter line on a darker background, negative for a dark line on a light background, and zero if there is no line passing through that pixel in the scene image. These values of the feature at each pixel are arranged in the same format as the scene image values, forming a feature image for each feature.

A training process is required by this ATR system. This requires a set of images, one image from each imaging sensor, for each of several training scenes. The system will work with as few as one training scene, but better results might be expected from the use of many, perhaps hundreds, of training scenes. The set of training scenes must include at least one target and some background. A knowledgeable person must examine each training scene and for each scene create a mask. The mask is a set of data with the format of an image ( 1 byte per pixel, for example), but with each pixel value being 2,1 , or 0 indicating whether that pixel is part of a target, background, or unspecified region in the training scene. Thus, the entire set of training data comprises several real images and one mask "image" for each of several, or possibly only one, real world training scenes.

In addition to designating target and background regions in the training scenes, a skilled operator must select an initial set of local features that might distinguish. between the target and background regions. If this operator-selected initial set of features does not include features that can distinguish target from background, the ATR programs will still run, but the unce:tainty in target identification will be large, perhaps so large that the ATR system results will be useless. If, on the other hand, the operator-selected initial set of features includes more features than are necessary to distinguish target from background, the ATR system will tell the operator the importance of each feature so that the operator can discard unimportant features and make the final process more efficient. The only disadvantages of an operator selecting too large a feature set initially are some computational inefficiency in the training process (which is probably tolerable) and, in the extreme case, the inability of the computer programs to handle so many features. (In principle, the formalism can handle any number of features, but any particular computer program will be limited.) Thus, although great skill is not essential in selecting the initial set of features, a clever choice can make the ATR process more efficient and effective. 
The third and final task of the operator during the training process is to select a value for the relative weighing of two types of errors. A type 1 error is the incorrect designation of a background region as a target by the ATR system; in a type 2 error, a target is wrongly called background. The ATR system could make either of these two error rates as small as desired, at the expense of making the other error rate larger. We would like to make both error rates small simultaneously but that may not always be possible. The procedure used here is to form a weighted sum of the two error rates, with weights $W_{1}$ and $1.0-W_{1}$, where $0<W_{1}<1$, and to minimize this weighted sum. The $W_{1}$ value is the relative weight of type 1 errors; type 1 errors are considered more important if $W_{1}$ is larger (closer to 1 than to 0 ).

The ATR training process takes as input (1) the training images and masks, (2) the definitions of the operator-selected feature set, and (3) the value of the type 1 error weight $W_{1}$. The training process then calculates values for coefficients, optimized to minimize the total (weighted sum) error rate. These coefficients are used in the production process (to be mentioned shortly) to indicate how much of each feature should be included in an analysis that attempts to identify targets in a scene. The training process indicates the importance of each feature and an estimate of the total error rate. This training process is done for the complete feature set specified by the operator, for the subset in which the least important of the original features is removed, for the best subset of this subset, and so on for all successive best subsets of features until the smallest subset is too small to allow a calculation (usually, one feature). This allows the operator to select an efficient subset of features with an acceptably low error rate, if such a subset exists. Or, in the worst case, the operator must recognize that this ATR system, with the feature sets attempted, cannot distinguish the targets from the background.

One product of the training process is the magnitude of the minimized total error rate. This is the estimated uncertainty in the ATR system for this particular set of features and coefficients for this type of image data. This is a prediction of the fraction of the pixels that will be incorrectly identified as target or background by the production process.

After an acceptable feature set is found, the coefficients for that feature set are used in setting up the production or surveillance program part of the ATR system. Then, a set of images (no mask) from any scene is input to the production program, and the program tells whether there is a target in the scene. This production process does not require any special skill on the part of the operator. Since this production process takes multiple images as input and yields one single result image as output, it accomplishes image data fusion, or the simultaneous analysis of several input images to produce a single result.

\subsubsection{Mathematical Overview}

5.5.4.1 Production Process. The production or surveillance process is used to scan large amounts of data with minimal expert interaction. This process accepts a set of $\mathrm{T}$ images of one scene as input, where $T$ is any value greater than 0 . From the $T$ scene images, we calculate $F$ feature images $F_{s, f}$, where $s$ is the scene number, and $f$ is the feature number, $f=1,2, \ldots F$. The user has decided beforehand the number of feature images $F$ and the nature of each feature image. Each feature image is multiplied by a coefficient $C_{f}$ whose value has been determined in a training process, and these scaled feature images are then added to obtain a result image $\mathbf{R}_{\mathrm{s}}$ for this scene s: 


$$
\boldsymbol{R}_{s}=\sum_{f=0}^{F} C_{f} \boldsymbol{F}_{s, f} \text { for each scene s. }
$$

If a pixel in the result image $\mathbf{R}_{\mathrm{s}}$ has a value greater than a certain value $Q$ determined from the training process, that pixel is called a target pixel; otherwise, the pixel is called a background pixel. Thus, the result image classifies every pixel in the scene as either target or background. This production process is simple in principle, although the calculation of some of the feature images may be complicated in practice.

5.5.4.2 Training Process. The values of the coefficients $\mathrm{C}_{\mathrm{f}}$ and $\mathrm{Q}$, which are used in the production process, are determined by a training process. This training process uses $S$ training scenes, indexed $s=1,2, \ldots S, S>0$. For each scene, there are $T$ training images, indexed $t=1,2, \ldots T, T>0$, and one three-level mask image. The mask pixel values are 2 for target regions, 1 for background regions, and 0 for unspecified regions. These regions have been selected manually by some knowledgeable person. The $T$ training images are assumed to be in registration. Let $T_{s, t}$ be the training scene images, and let $\mathbf{M}_{s}$ be the mask, for each scene s.

For each scene $s$, we extract $F$ feature images $F_{s, f}, f=1,2, \ldots F$, from the $T$ scene images. The algorithms used to construct feature images $F_{s, f}$ do not depend on $s$; the feature images are constructed in the same way for every scene. The algorithms do depend on $f$, and in fact, the algorithms must be different for each different $f$; if two different feature images (that is, with different $f$ values) equal one another in every training scene, the mathematical procedures become indeterminate and the coefficient optimization procedure cannot be done. We define a result image $\mathbf{R}_{s}$ for each training scene s:

$$
\boldsymbol{R}_{s}=\sum_{f=0}^{F} C_{f} \boldsymbol{F}_{s, f} \text { for each scene } s=1,2, \ldots . S
$$

The coefficients $C_{f}$ are to be determined in this training process. The $C_{f}$ are the same for all. Ideally, we would like to choose values for the $C_{f}$ to make $R_{s}$ equal to $\mathbf{M}_{s}$ in regions 1 and 2 (we do not care about region 0), for every s. That is, we would like to use the pixel values in $\mathbf{R}_{s}$ as indicators of whether the pixel is part of a target (value equal to or close to 2) or a background region (value equal to or close to 1). We do not expect to be able to accomplish this exactly. In practice, we can usually choose a set of coefficients to make $\mathbf{R}_{\mathrm{s}}$ approximately equal to $\mathbf{M}_{i}$, with only a few pixels in $\mathbf{R}_{\mathrm{s}}$ giving a misleading indication of target or background. The criterion for optimizing the values of the coefficients $C_{f}$ is minimizing of the number of these incorrectly classified pixels in the training data. This minimization calculation is not difficult, but it requires the assumption of some analytical form (we chose a Gaussian function) for the distribution of the differences between corresponding $\mathbf{R}$ and $\mathbf{M}$ pixel values. The mathematical details are found in Partin et al. (1994).

\subsubsection{Application To Digface Data Interpretation}

The ATR system was applied to digface characterization project data taken in 1993 (Josten 1995). The purpose of the data collection activity was to evaluate the cligface characterization concept when 
sensing isolated objects at several engineered characterization cells where objects had been buried in known surveyed locations. Details of the experiments are found in Appendix A, taken from Josten (1995). The following description is a summary of the experimental data used in evaluation of the ATR system.

Each experiment comprised a single positional setting of the trolley used to deploy the different sensors over the buried objects. Refer to the figures in Appendix A. For each experiment (six total), the sensors were scanned in planar fashion over the objects. Each planar scan resulted in an $\mathrm{x}, \mathrm{y}$ data set for constant $\mathrm{z}$ (distance from the objects). For each setting (experiment), however, the $\mathrm{z}$ axis was also changed so that the $x, y$ planes were obtained at different levels. Typically, the different levels of data were obtained at 6 in. increments in the z-direction.

For evaluation of the ATR system, data from five experiments were used, referred to as E1, E2, E3, E5, and E6. (Experiment 4 was designed for evaluating plume detection at the digface and was not used.) The different experiments represent data recorded over five different areas with different buried objects. The five rectangles on the left of Figure 5-14 indicate the approximate sizes and locations of buried objects in the five experiments, as seen from above. In these sketches, the solid objects are magnetic, and the objects drawn with outlines only are mostly not magnetic. In E2, for example, there are a magnetic barrel (object 7) and a wooden box (object 6). Objects 9 and 10 in E3 are two boxes, one above the other, with some magnetic material in the top box. The top box was removed between levels L3 and L4 (levels are described immediately below). Similarly, object 20 (a vertical barrel) in E5 was removed between L2 and L3, and object 27 (a vertical steel pipe) in E6 was removed between L2 and L3.

For each experiment, the different levels used are referred to as $\mathrm{LO}, \mathrm{L} 1$, etc., where $\mathrm{LO}$ is the highest level, and the sensors are 6 in. lower (closer to the buried objects) for each successive level. In some cases, layers of soil were removed between successive measurement levels. Any one level of any one experiment comprises a scene. Different scenes are expected to give different results for any measurement because they contain different objects, or because the objects are at different distances from the sensors. For each scene, as many as seven sensors acquired different types of data. The sensor designations are shown in Table 5-8. Data from the volatile organic sensor were not used in this study.

For this analysis, the readings for each sensor for each scene were arranged in image format, two-dimensional arrays having 43 columns ( $43 \mathrm{x}$-values, $\mathrm{x}$ being positive southward) and 25 rows ( 25 $y$-values, $y$ being positive eastward). Some of the data sets did not have this many rows or columns, and some were missing a few data points from what was expected to be a regularly spaced array. In all of these cases, the missing data points were filled in using a linear interpolation or extrapolation procedure so that each data set used in this study had a full $1,075(43 \times 25)$ data points.

The system was evaluated for its capability of detecting two separate classes of targets: barrels and boxes.

5.5.5.1 Identification of Barrels, Linear Analysis. A simple linear analysis was performed using a linear combination of the six raw data images (corresponding to an image from each sensor) and augmented with the "constant" image, which is always included for mathematical completeness. The training was done with three scenes: E2L7, E5L5, and E6L5. The masks used were M2A, M5B, and M6A shown in Figure 5-14. In this figure, the red (actually more brown) areas of the masks are 


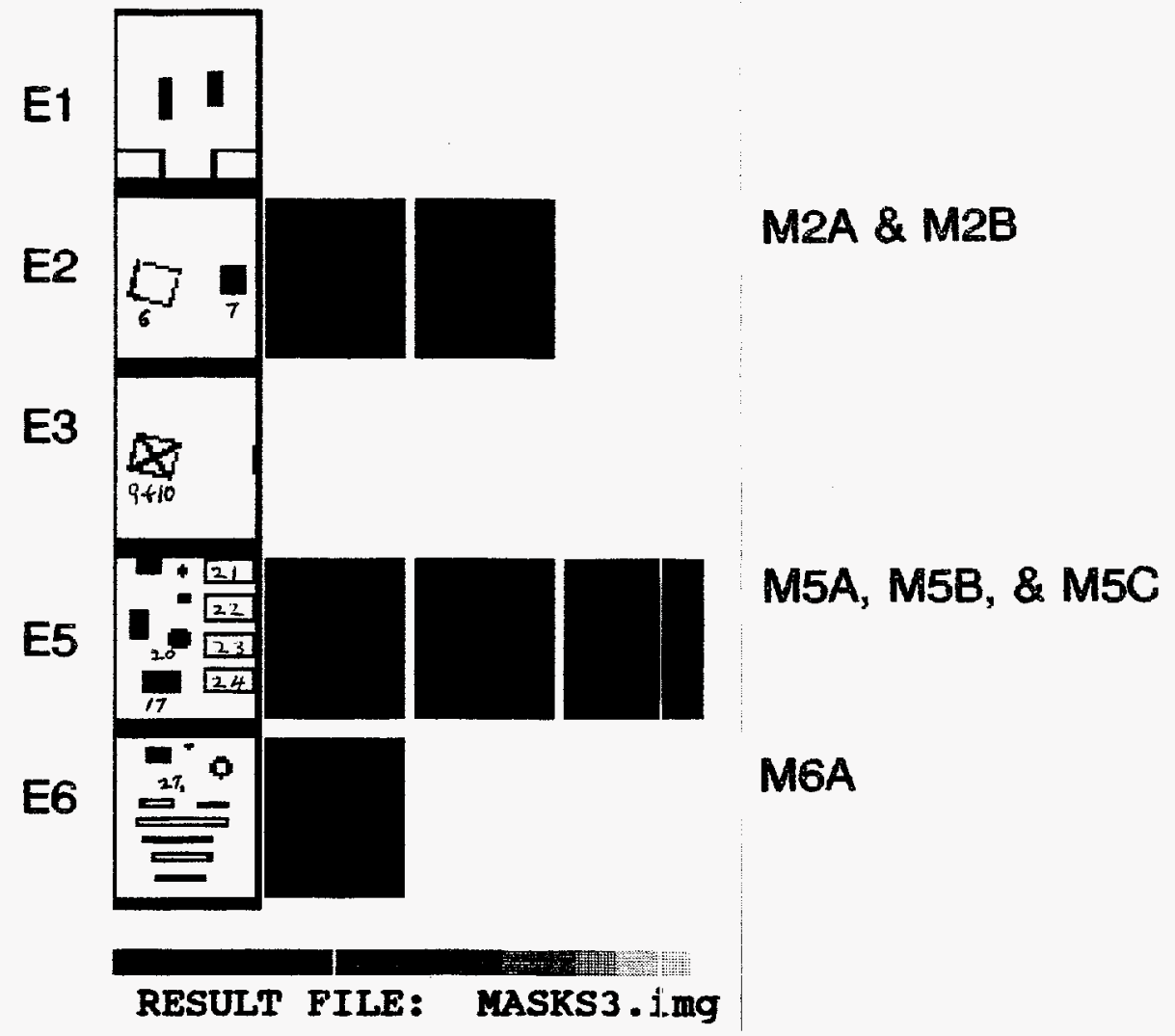

Figure 5-14. Buried objects and masks used in training. 

Table 5-8. Digface characterization sensor description.

\begin{tabular}{cl}
\hline Sensor & \multicolumn{1}{c}{ Description } \\
\hline 1 & Magnetic field, vertical component and vertical component gradient \\
2 & Ground electromagnetic (EM) conductivity, horizontal dipole, quadrature phase \\
3 & Ground EM conductivity, horizontal dipole, inphase \\
4 & Ground EM conductivity, vertical dipole, quaclrature phase \\
5 & Ground EM conductivity, horizontal dipole, inphase \\
6 & Volatile organic compound sensor \\
\hline
\end{tabular}

designated as background, the green areas are target, and the black areas are not used in the training process. The objects identified by the masks in this study are identified in Table 5-9.

After training on the three scenes with the three masks described, the system was used to analyze 30 scenes from Experiments E2, E3, E5, and E6. (Experiment E1 was not included in the analysis because only four sensors, instead of six, were used in Experiment E1.) The results of the application to the 30 testing scenes are shown in Figure 5-15. In this figure, as in any of the result images, lighter regions are stronger indications of targets, and darker regions are stronger indications of background. Green and white (i.e., very light green) indicate what the system has classified as targets. Red and brown and black (dark red) indicate what the system classifies as background. Figure 5-15 shows that the analysis did correctly identify the barrel (object 7) in E2 and another barrel (object 17) in E5 as targets when the excavation was deep enough so that the sensors were close to the targets. The process also incorrectly gave small target indications at the top of E5L5 and E6L6. These correspond to ferromagnetic objects other than barrels, so it is not surprising that the simple linear combination algorithm did not distinguish these objects from magnetic barrels.

The linear analysis algorithm also failed to detect a barrel (object 20, vertical 55-gal empty barrel) at the center of E5, in levels L0-L2 (this barrel was removed between levels L2 and L3). The reason for the failure is unknown. However, the barrel shows rather weakly in the raw data, suggesting the possibility that it may have been buried too deeply for detection using this algorithm.

Although the linear combination algorithm did not yield perfect results, it did well considering the simplicity of the algorithm and the fact that the algorithm required and used no understanding of the measurements or the physical processes involved.

In an attempt to increase understanding of performance, the above processes were repeated with an additional training scene, E5L2 with mask M5A, which specifies the troublesome barrel (object 20) be designated a target in the training process. The results of analysis after the new training set is shown in Figure 5-16. The system now located object 20, but it also incorrectly identified a box (object 6) and other objects as targets. This rather poor performance indicates that the simple linear combination algorithm cannot reliably distinguish between objects designated as targets and those designated as background in this training set. The problem is that object 20 , a barrel, looks to the sensors like some 
Table 5-9. Automatic target recognition mask object relationship.

\begin{tabular}{lcll}
\hline Mask & $\begin{array}{c}\text { Object } \\
\text { number }\end{array}$ & \multicolumn{1}{c}{ Object description } & $\begin{array}{c}\text { Depth } \\
(\mathrm{ft})\end{array}$ \\
\hline M2A & 7 & 55-gal barrel, containing foam & 5.2 \\
M2B & 6 & Wooden box and surrounding area & 5.1 \\
M5A & 20 & 55-gal barrel, vertical, empty & 3.1 \\
& 17 & 55-gal barrel, horizontal, empty & 3.9 \\
M5B & 17 & 55-gal barrel, horizontal, empty & 3.9 \\
M5C & 21 & Wooden box, empty & 3.6 \\
& 22 & Wooden box, containing dry dirt & 3.7 \\
& 23 & Wooden box, containing wet dirt & 3.7 \\
M6A & 24 & Wooden box, empty & 3.7 \\
\hline
\end{tabular}




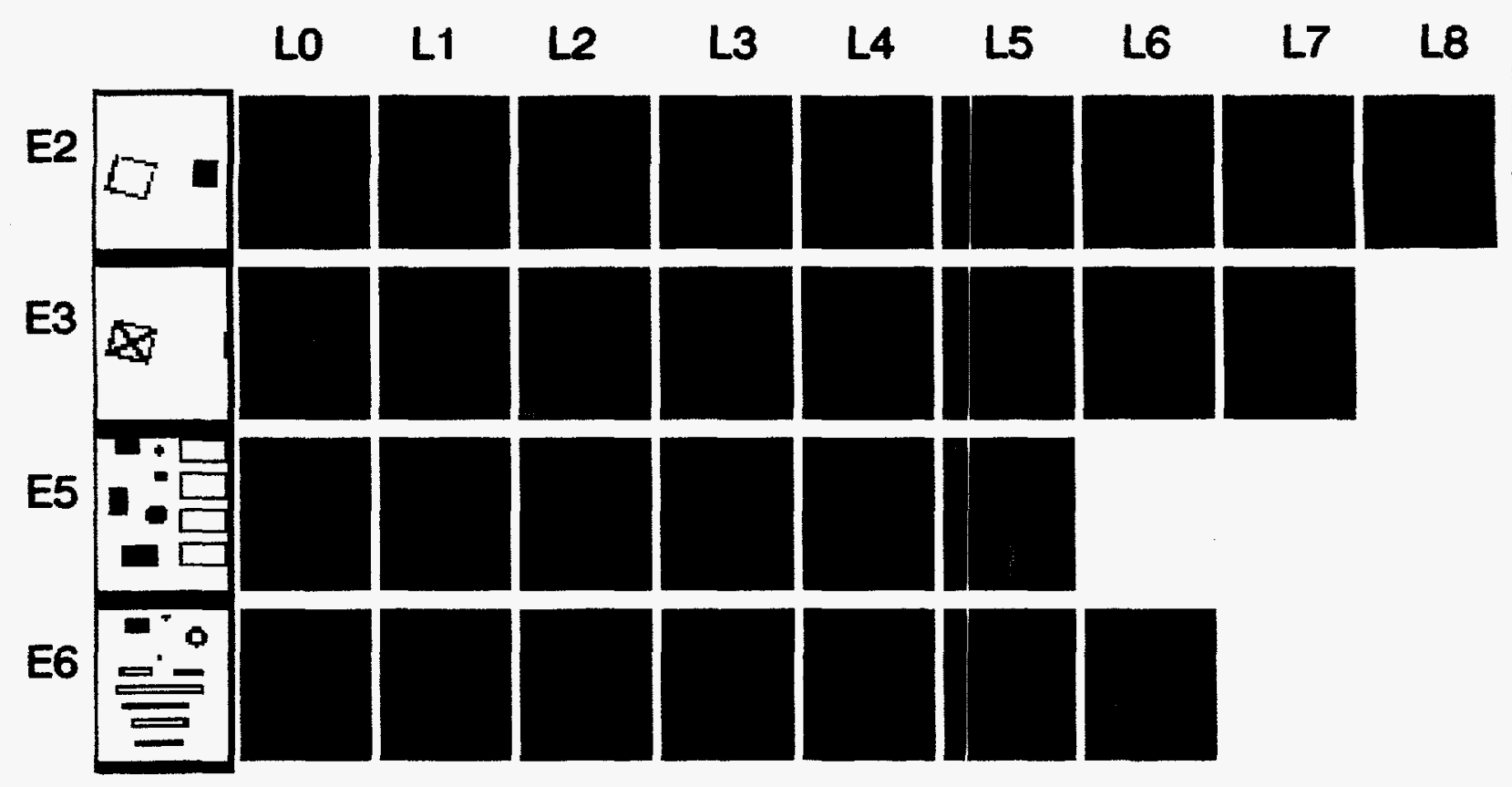

RESULT FILE: comlA3.ing

Figure 5-15. Application of system trained on three scenes to target detection in 30 testing scenes. (Levels are approximately 6 in. apart. Level 0 is approximately 2, $\mathrm{ft}$ abovegrade.)

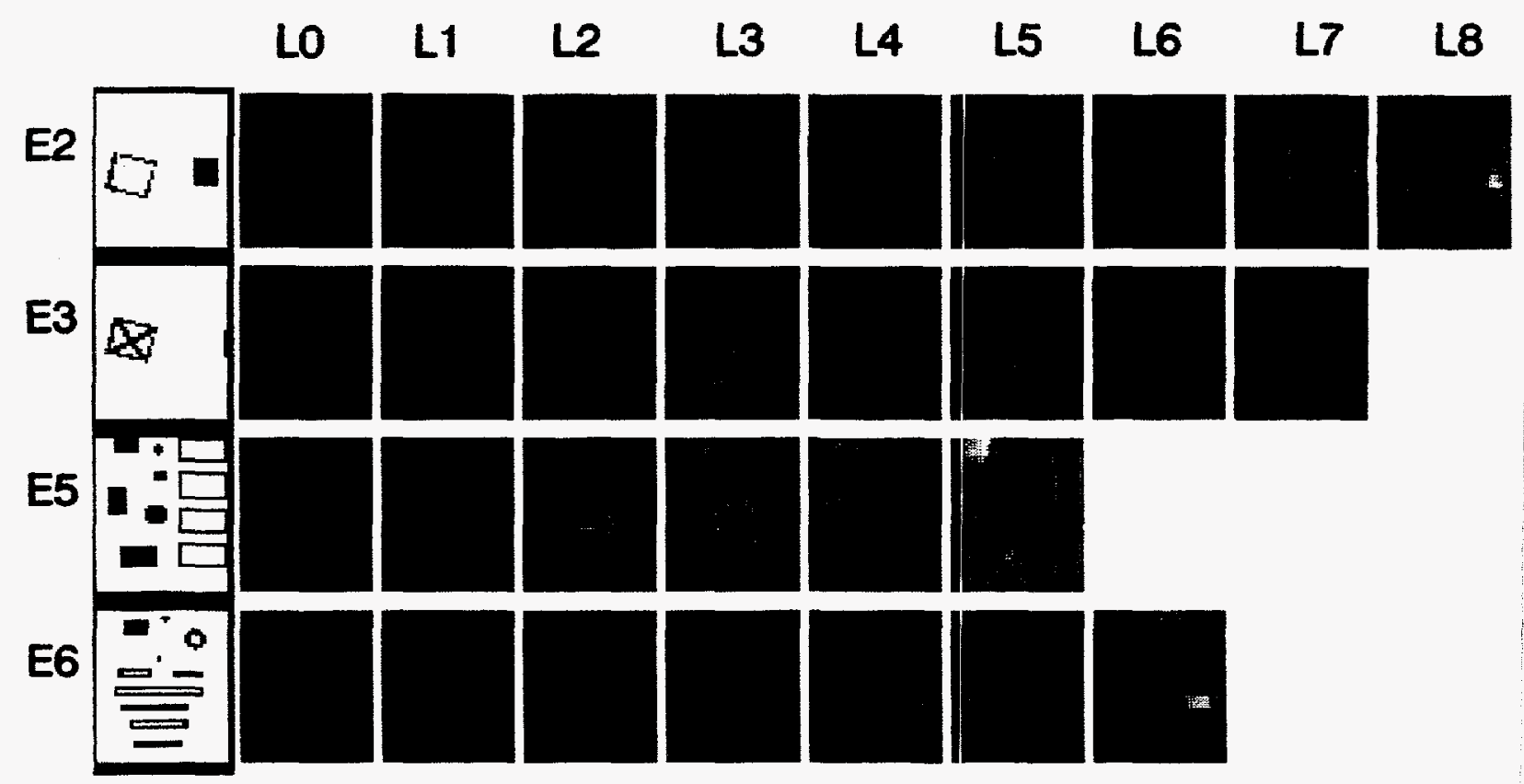

RESULT FILE: com4G4.img

Figure 5-16. Search for barrels, linear combination, four training scenes. (Levels are approximately 6 in. apart. Level 0 is approximately $2 \mathrm{ft}$ abovegrade.) 


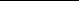


other background object more than a target. By including it in the training set, the likelihood that other nonbarrels will be identified as targets is increased. The identification problems with the simple algorithm led to the conclusion that a better algorithm should be tried.

5.5.5.2 Identification of Barrels, Quadratic Analysis. The next level of complication in analysis was to add quadratic terms to the linear analysis, adding 21 product terms to the linear and constant terms in the previous analysis. This was tried using the same four-scene training data set as described for the linear case, with the results shown in Figure 5-17. As expected, the quadratic analysis worked better than the linear analysis at distinguishing barrels from background, but it is still not perfect. In E3, the stacked boxes are incorrectly identified as targets, and in E6, the truck V8 engine is also incorrectly identified as a target.

5.5.5.3 Identification of Boxes, Quadratic Analysis. The same quadratic procedure was used to try to find boxes as targets instead of barrels. In this analysis, the training process used two training scenes, E5L5 with mask M5C, and E2L7 with mask M2B. These masks select only boxes without ferrous metal contents as targets. The results of this analysis for 30 scenes are shown in Figure 5-18. This analysis was repeated using only the first training scene with results shown in Figure 5-19. Comparison of the figures shows that the results of this type of analysis can depend significantly on the choice of training data. In both of these two analyses, boxes (objects $6,9,10,21,22,23$, and 24) were correctly identified as targets, and a few other objects were incorrectly identified as targets, with the single-training scene a little better than the two-training scene results. Overall, the quadratic analyses results were good.

5.5.5.4 Identification of Barrels, Using Peak Width of Magnetic Data. The linear and quadratic analyses did not rely on any physical understanding of the sensor measurements. To more accurately identify barrels, we tried a more sophisticated analysis using the measurement processes. In this analysis, we tried to find peaks in the magnetic field and its gradient and to discriminate on the basis of the widths of the peaks, on the assumption that barrels should cause peaks of a certain width in magnetic field measurements. This analysis used only two sensors, comprising the vertical component of the magnetic field and vertical component of the field gradient. The training process used the same training scenes as were used for the quadratic function search for barrels (E2L7 with mask M2A, E5L5 with mask M5B, E6L5 with mask M6A, and E5L2 with mask M5A). The results of this analysis are shown in Figure 5-20. Experiment 1 is included because this analysis does not require the sensors that were not used in Experiment 1. The analysis was repeated with a different set of training data, using scenes E2L8, E5L2, E5L5, and E6L6 with the same masks as before. This gave slightly better results, as shown in Figure 5-21. The results generally identify the barrels as targets, which is good. They also show other ferromagnetic objects as targets, which we had hoped to avoid. However, we are not able to distinguish well between barrels and other magnetic objects, because the peak width, used as the distinguishing feature, is not really unique to barrels. This analysis also shows as targets some presumed nonmagnetic objects such as a box (object 6) in E2. This is a strong indication that these boxes do in fact include some ferromagnetic material.

\subsubsection{Discussion}

The system performance was generally good in the data interpretation application. The system was able to do quite well at finding buried objects, and it could give some indication of the nature of the 



\section{$\begin{array}{llllllllll}\text { LO } & \text { L1 } & \text { L2 } & \text { L3 } & \text { L4 } & \text { L.5 } & \text { L6 } & \text { L7 } & \text { L8 }\end{array}$}

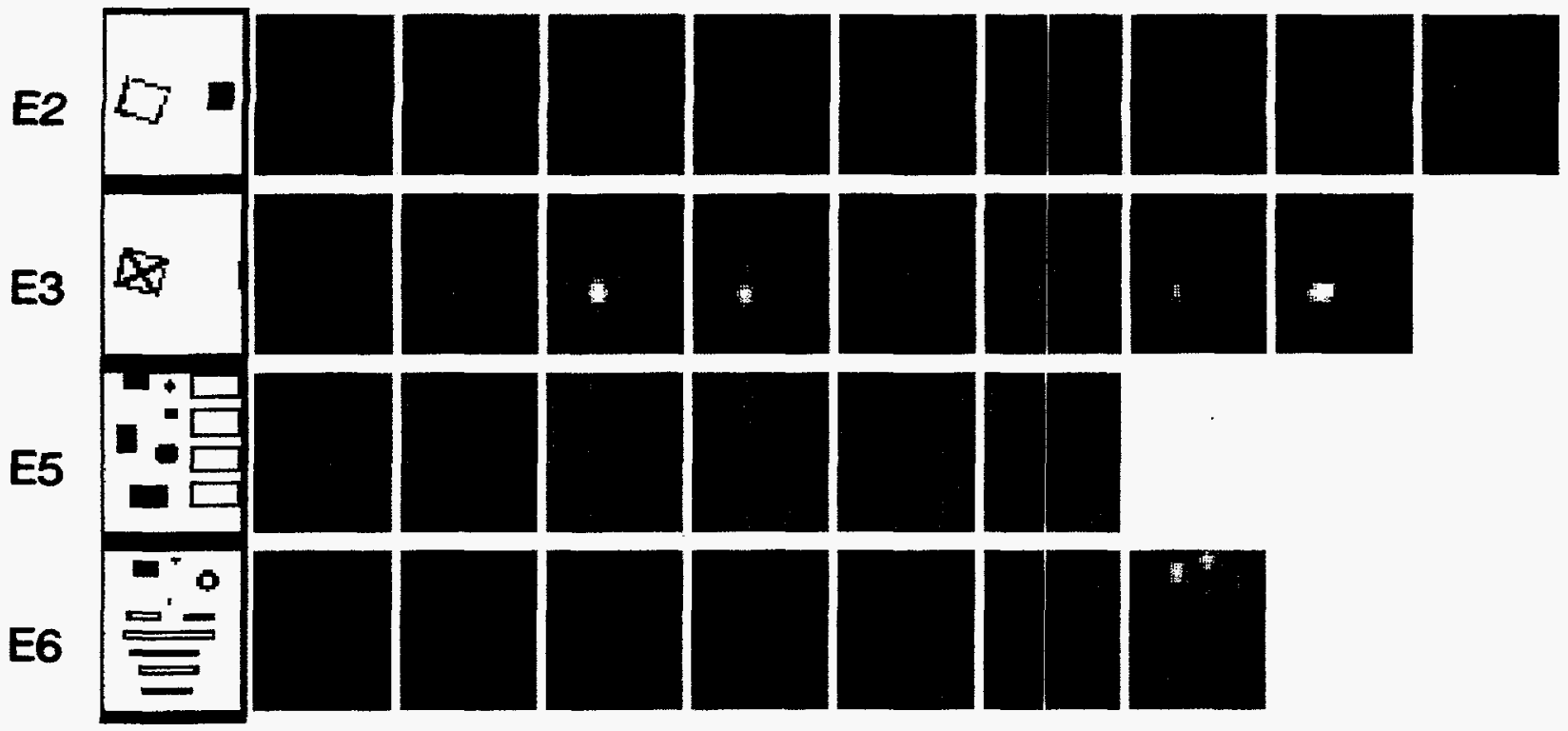

RESULT FILE: \ri\com4i3.ing

Figure 5-17. Search for barrels, quadratic combination. (Levels are approximately 6 in. apart. Level 0 is approximately $2 \mathrm{ft}$ abovegrade.)

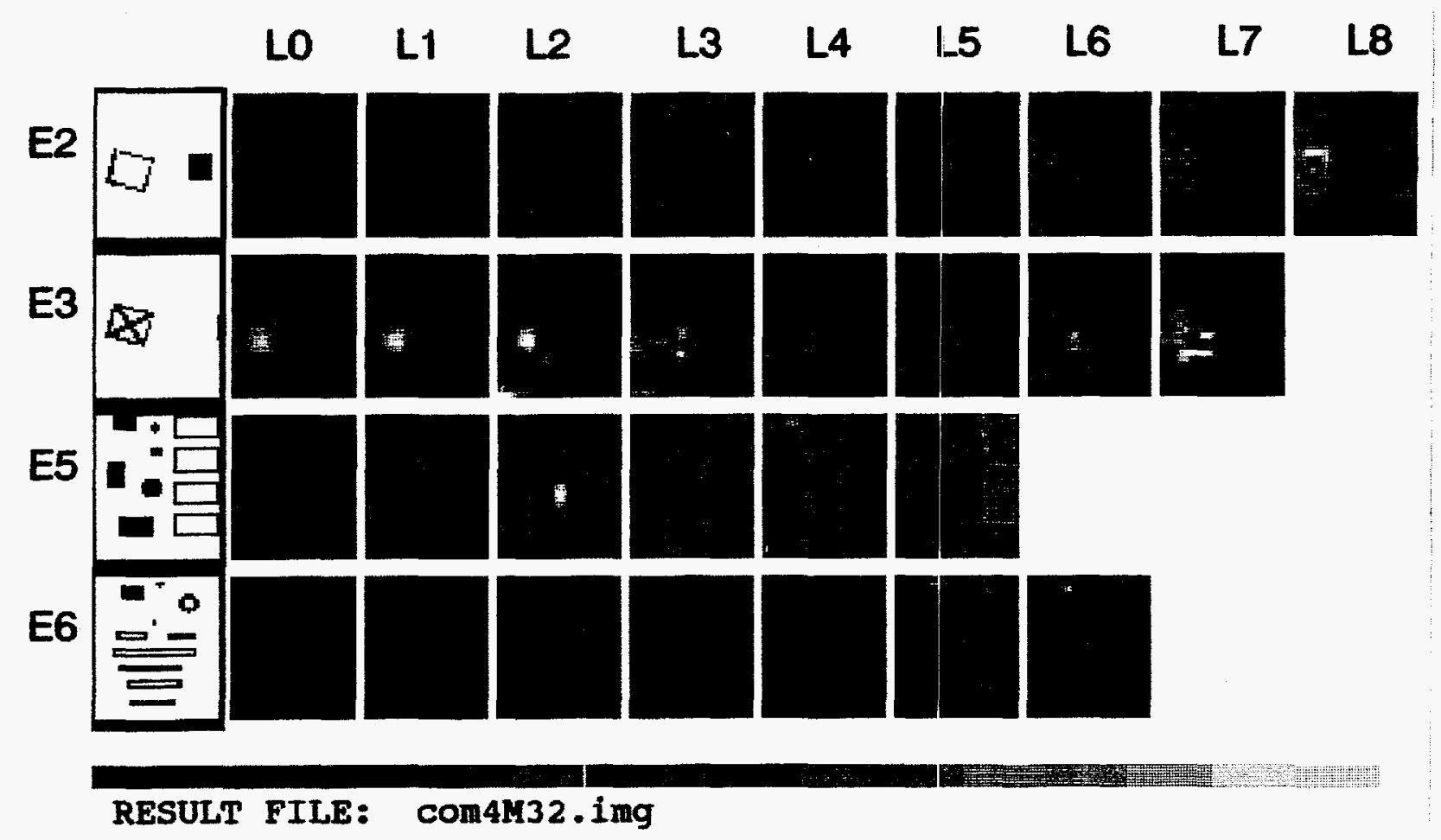

Figure 5-18. Search for boxes, quadratic, two training scenes. (Levels are approximately 6 in. apart. Level 0 is approximately $2 \mathrm{ft}$ abovegrade.) 



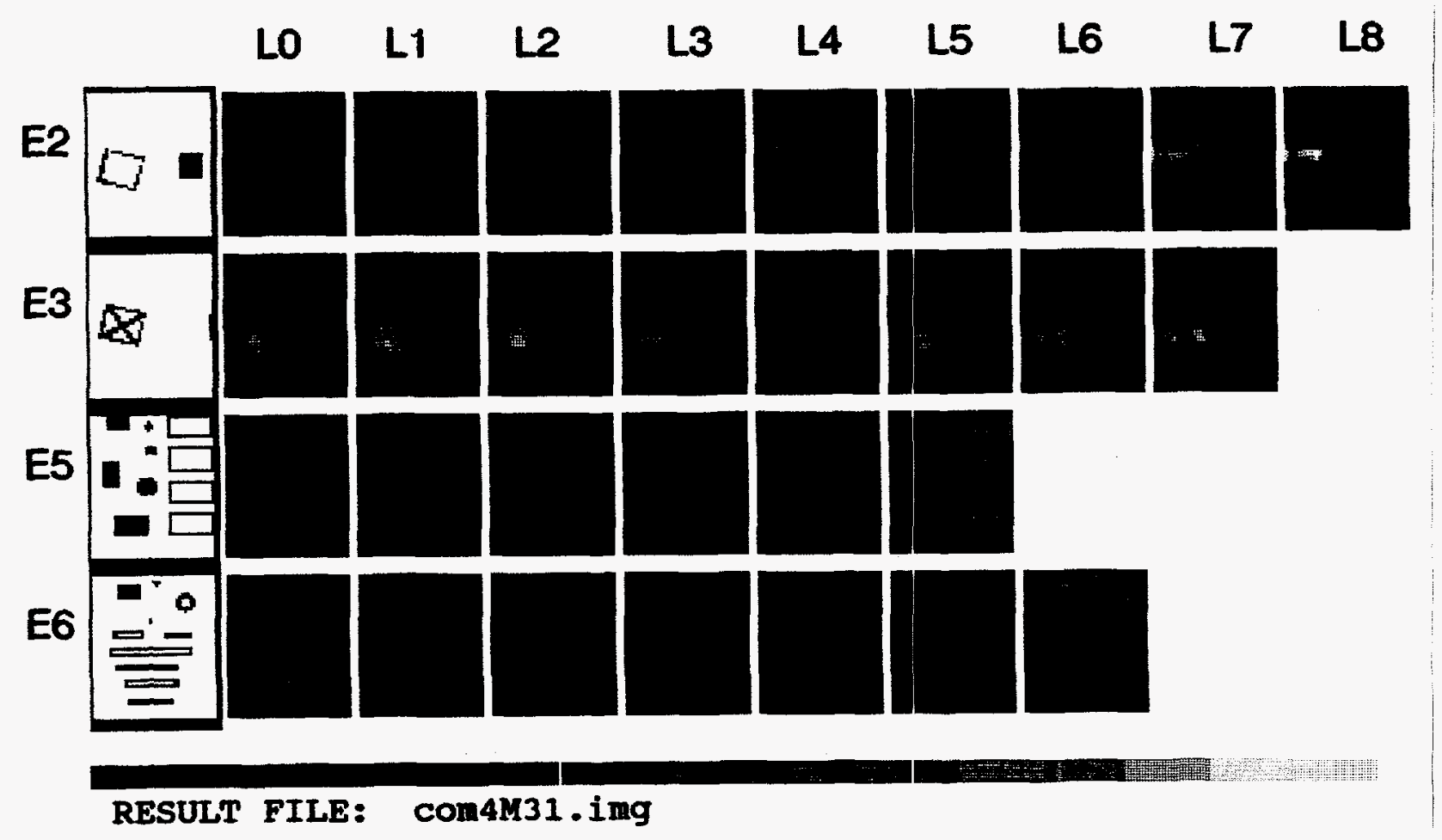

Figure 5-19. Search for boxes, quadratic, one training scene. (Levels are approximately 6 in. apart, Level 0 is approximately $2 \mathrm{ft}$ abovegrade.) 


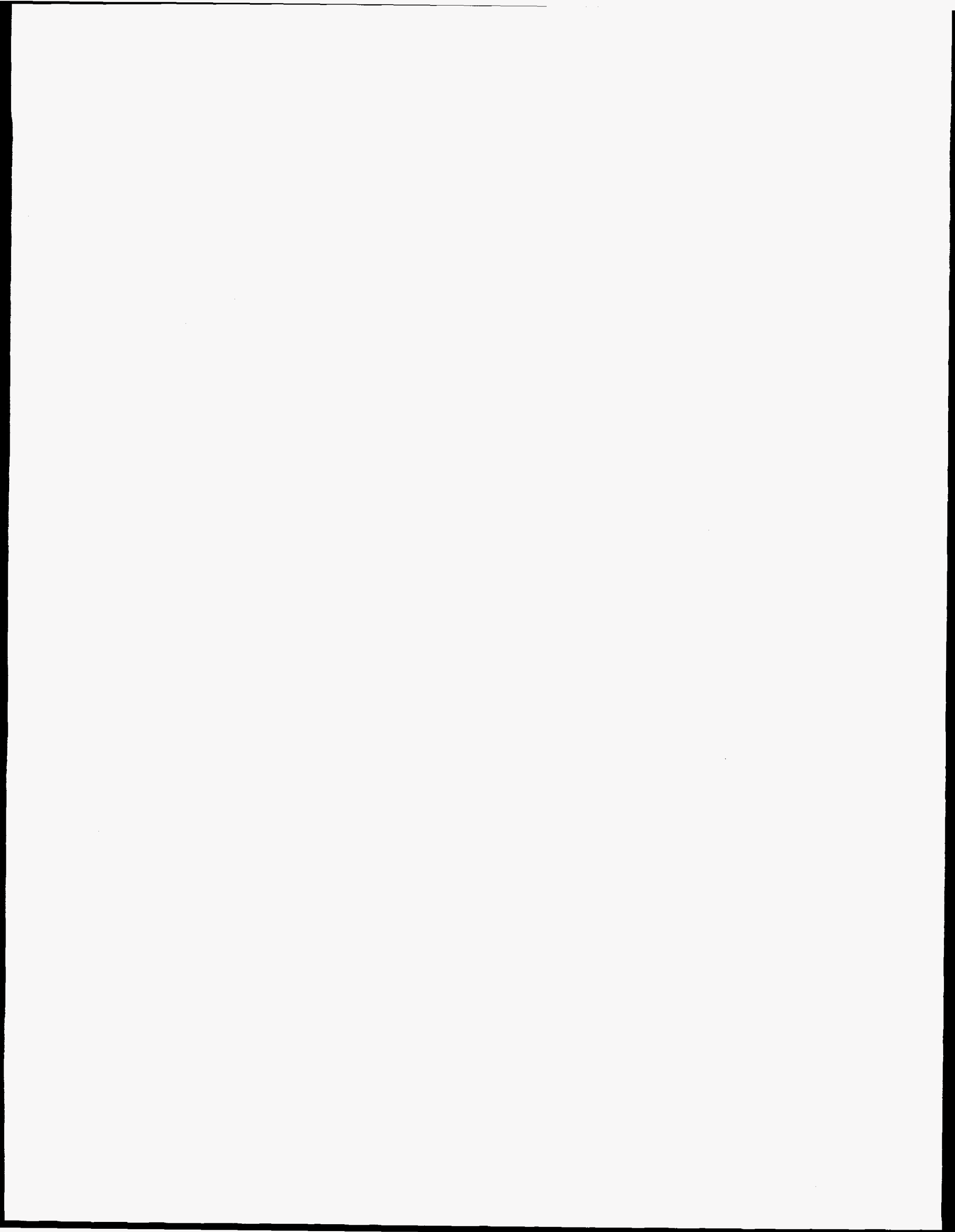




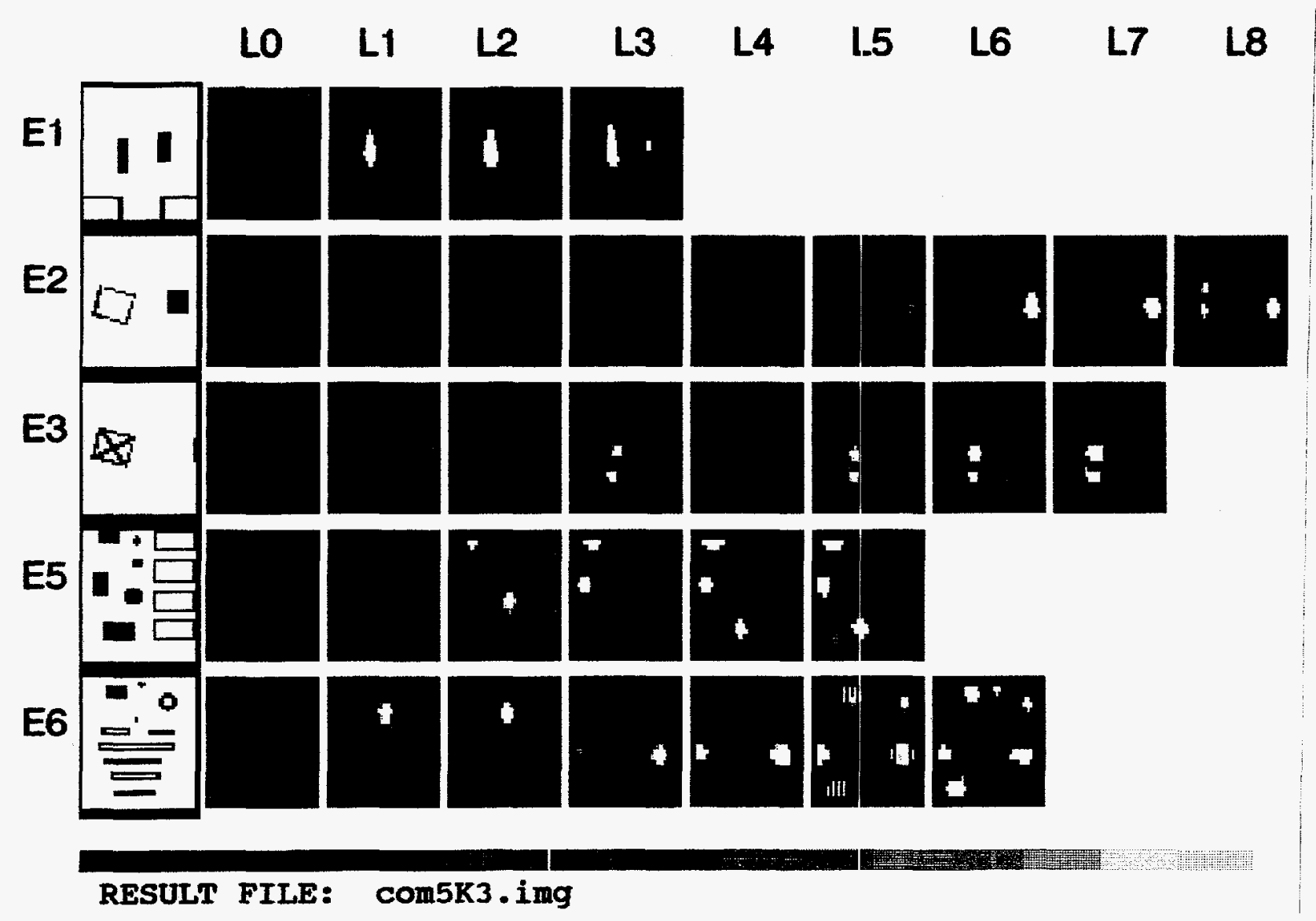

Figure 5-20. Search for barrels, peak width, first training set. (Levels are approximately 6 in. apart. Level 0 is approximately $2 \mathrm{ft}$ abovegrade.) 


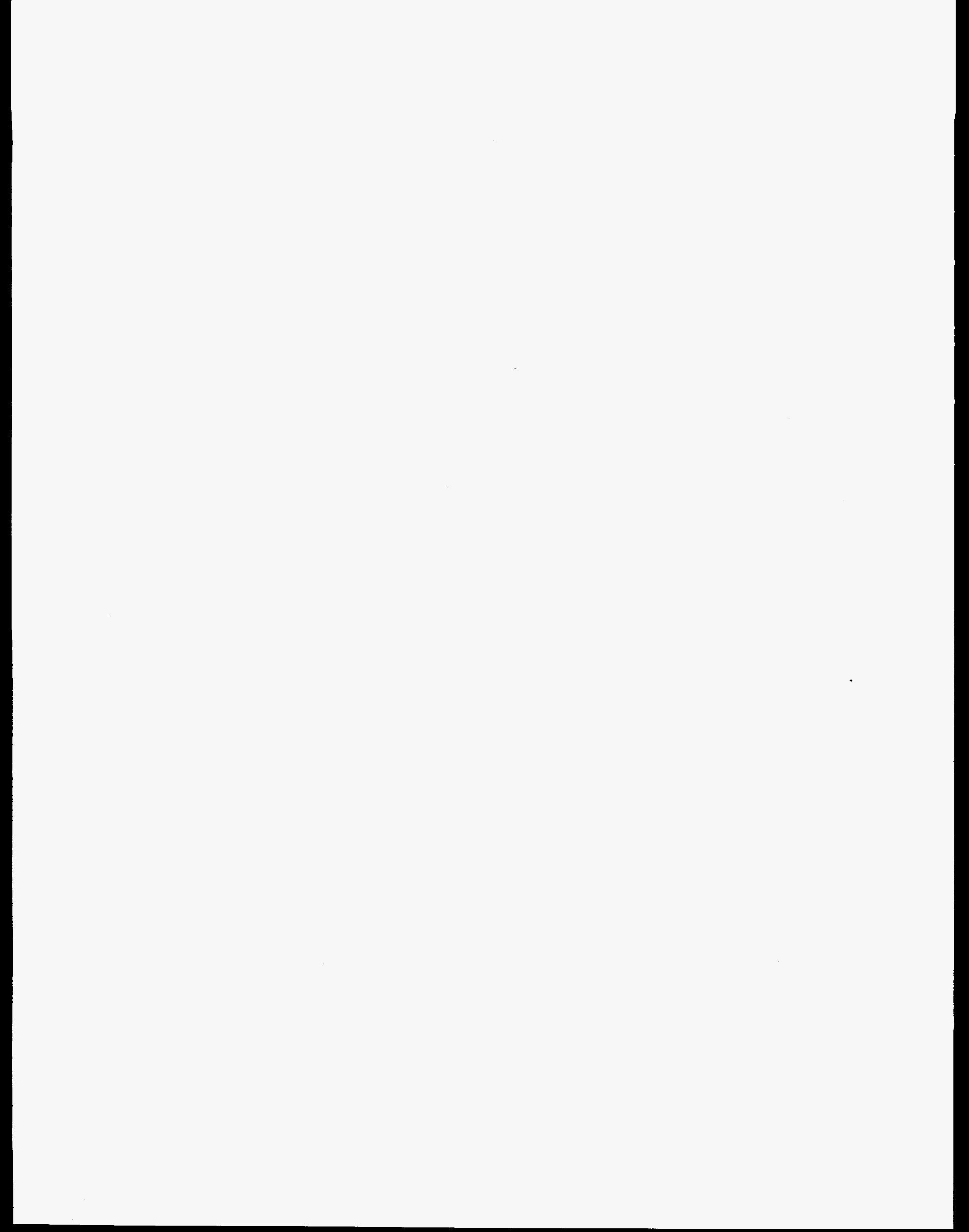




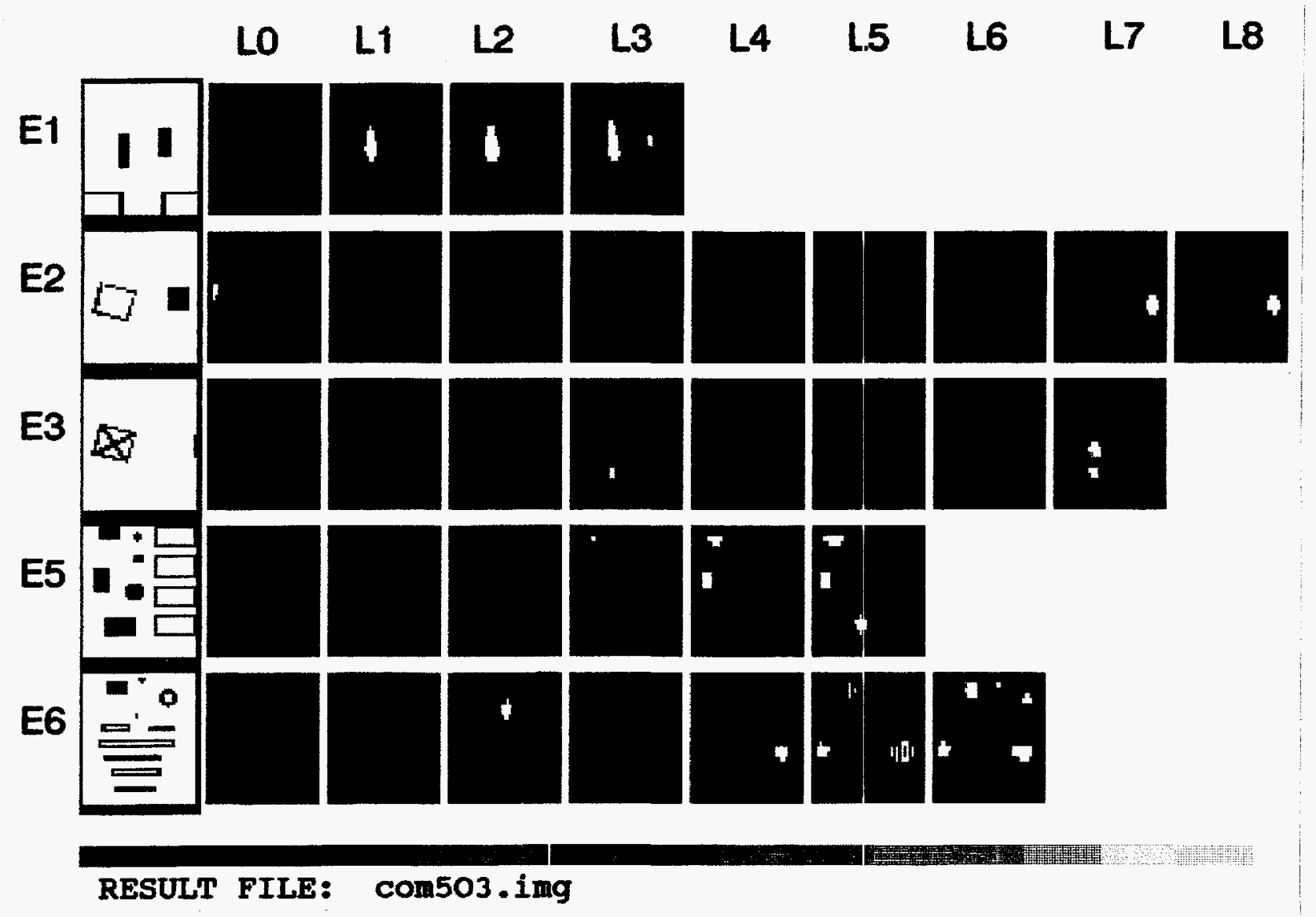

Figure 5-21. Search for barrels, peak width, second training set. (Levels are approximately 6 in. apart. Level 0 is approximately $2 \mathrm{ft}$ abovegrade.) 

object (magnetic or not) and the depth of the object (relatively shallow objects are detected by the system). Failure to do better at indicating object type or depth is primarily due to limitations in the type of data available. Additional sensor data, as discussed below, mily improve the system's capability in identifying targets of interest. At the same time, before making improvements, additional thought should be given to defining the requirements of a target recognition system for buried waste identification. The data collected in 1993 to which the system was applied consisted of defined targets discretely placed (Josten 1995).

The intent of this field testing activity was to define the feasibility of the digface concept and demonstrate, empirically and definitively, the improvement in target resolution as sensors are brought closer to the target and data are collected at a fine grid spacing. The application of the target recognition system was consistent with manual human data interpretation in that the definition of targets clearly improves as the sensors are brought closer to the waste.

The next step in demonstrating the digface characterization concept is to collect data for randomly disposed waste representative of real waste at the INEL and other DOE sites (Josten and Roybal 1994). The target recognition system ultimately must be useful in dealing with data such as the additional data collected in 1994 by the digface project. The new data represents a more challenging problem because of the multifarious interference effects existing in randomly disposed waste. The data interpretation challenge is increased for both the human expert and any target recognition scheme. The new data should be evaluated by expert geophysicists, and ideas generated on what targets should be defined. It may be that more subtle, and possibly abstract, features (rather than boxe; or barrels) should be identified. In the waste seam of actual burial pits or trenches, the seam will generally be all target, where target is thought of as debris. Therefore, in the waste seam, it may be that classifying different waste regions makes most sense; examples of potential regions are given in Josten and Roybal (1993). These could include distinguishing zones on the basis of buried object size, amount of metal mass, or random versus orderly distribution.

After extensive human evaluation of data collected in 1994 for representative waste, a decision should be made on whether to apply the ATR scheme to evaluation of targets for such waste configurations.

Additional sensor data would likely improve the target recognition system's ability to recognize targets. The additional sensor information would improve human irterpretation as well. The geophysical sensors used [magnetometer and electromagnetic (EM) sensor] are responding to similar physical phenomena (EM properties) in the waste. Additional information from sensors responding to "orthogonal" characteristics would possibly improve performance significantly. Such sensors might include gravity or ground penetrating radar (GPR), which cculd respond to density differences irrespective of EM properties. In regard to potential new sensors, it is important to consider actual deployment in anticipated remediation scenarios. There will always be pressure to obtain maximum amount of information from sensors that can be easily and quickly deployed at the digface.

The system has several limitations. First of all, in an image scene to be scanned the system requires data from all grid points that were included in the training data. The occurrence of missing data points is virtually certain in realistic applications. However, in practice, this limitation is not seriously restrictive because missing data can be interpolated (or extrapolated) from the neighboring real data; this technique was used during this study itself when missing data points were encountered. A second 
limitation of the present system is more serious. The system, once trained with data from specific sensors, expects to see the same complete set of data from each sensor in the image data containing the targets to be identified. If data from one or more sensors is missing, the system does not perform satisfactorily. Theoretically, the system could be retrained on the subset of sensors and applied to the data, but this would not be a realistic alternative under most circumstances. The expectation of missing sensors (due to failure or other unforeseen circumstance) is realistic. A reasonable approach to adapting the system to perform at a reasonable level in such situations is necessary for the digface data interpretation application.

In this initial application, the system worked on only one level of data at a time. This means that a single level of data was used in both training and surveillance modes. However, in theory the system could be extended to work on information from multiple levels to identify targets. The use of information from multiple levels offers an extension of potential interpretation capability as discussed above in Section 5.4. Evaluation of the system capability when trained on information from different levels should be performed. This would provide valuable insight into the use of multiple level information for digface interpretation; this insight may apply to human interpretation as well. It is possible that simultaneous data interpretation using information from multiple levels may be difficult for a human interpreter to do effectively when encountering large amounts of data in field conditions. If so, the ATR system might assist by screening data using multiple level information and then directing human attention to suspect areas. One issue to be addressed in using the system with multiple level data are the fragility of the system if the exact form of the data expected and used in the training set is not available at all times. This is analogous to the missing sensor data problem discussed above, except that in the new mode, all sensor data at all levels used in training must exist in the surveillance data mode. This issue would need to be addressed if the system is developed to use multiple levels of data.

The usability of the system in an integrated environment would increase if the software ran on the same workstation as the other VE tools. Work is in progress to port the software to a workstation. This would allow the target recognition capability to be integrated into the overall data analysis and display system. Limitations on speed or memory are potentially possible even in the workstation environment as the size of the files to be analyzed increases. The memory limitation is another aspect of the overall issue of data management that must be addressed as the VE system moves from a prototype tool working on relatively small data sets to a useful production tool robust enough to support operations in a real waste retrieval.

\subsubsection{Conclusions and Recommendations}

The following conclusions were drawn as a results of the evaluation of the ATR system:

- The ATR system performance was generally good for the data interpretation application. Although there was some errors in distinguishing boxes from barrels, the system is quite capable (based on the limited testing done) of distinguishing target objects from background soil.

- Results show that application of the target recognition system was consistent with manual human data interpretation in that the definition of targets clearly improves as the sensors are brought closer to the waste. 
- Initial results indicate that further evaluation and possible development of the ATR system are warranted. The reasonable success when searching for defined and resolvable targets needs to be demonstrated in environments representative of real waste sites.

The following recommendations are made:

- In future assessments, the ATR system training should be directed to distinguishing target from background only, as opposed to classifying specific types of targets. This capability (distinguishing target or background) is currently a higher priority than classifying target types.

- The limitations of the system in handling missing data (such as a sensor missing from the surveillance data that was present in the training data set) should be evaluated. If the system performs poorly without reasonable chance for improvement by development, the value in the digface application is small.

- An evaluation should be made of the system's potential to use volumetric data (information obtained from the same sensor at different levels, i.e., different distances from the targets search area). Based on likely digface application, information from two (or possibly three) levels should be considered. The evaluation should include assessing performance degradation when same level parameters vary in surveillance data from what was used in the training set. Inability to use multiple level data would not necessarily eliminate the ATR as a useful tool for the digface application.

- The ATR system should be evaluated for performance in representative waste environments. The most useful immediate evaluation should be done with the digface data collected in 1994 . However, before applying the ATR system, the 1994 data must first undergo extensive evaluation, and methods of human interpretation need to be devised.

\subsection{Neural Network Evaluation}

This work examined the applicability and feasibility of using artificial neural networks for examining and interpreting digface sensor data gathered by Josten (1995) (see Appendix A). Introductory remarks given in Section 5.5 indicate the logic for investigation of neural networks as well as the ATR technique. Details of this work are presented in Poulton and Brown (1994). The scope of work entails three major parts including classification of the data as target or background, estimation of depth of targets, and classification of the conductivity of the targets. Information on the training times, network designs, and sensitivities of the networks are included in the study.

Artificial neural networks have potential use for scanning large amounts of data rapidly to highlight regions of interest similar to those discussed for the ATR system evaluation. The regions of interest can then be shown to appropriate remediation personnel. The target recognition systems and artificial neural networks require training before the techniques can be applied. However, the two methods use different approaches, each with advantages and disadvantages. Thus, it is appropriate to evaluate the capabilities of both methods for support of digface data interpretation. 


\subsubsection{Background on Neural Networks}

Artificial neural network processing is a broad category of computer algorithms that solve several types of problems including classification, parameter estimation, parameter prediction, pattern recognition, completion, association, filtering, and optimization. A neural network is a computer simulation of the way animals use neurons to process and store information; it is a technique for solving problems, not a software in itself. Neural networks can be simulated with sequential software, electronic circuits, or optical circuits. For this study, a software realization of the networks was used. One aspect of neural networks is their ability to perform well in doing the same types of tasks at which humans traditionally have performed well, such as pattern recognition. Neural networks have not solved problems that have remained previously intractable to humans. In some cases, they provided faster, more accurate, more flexible solutions once a procedure has been learned.

Neural networks are not programs; they are procedures that solve problems by learning from other solutions. Typically a neural network is given a training set consisting of a group of examples from which it can learn. The most common training scenarios use supervised learning, during which the network is presented with an input pattern together with the correct answer, or correct classification of the input data. In creating pathways from one to the other of these paired examples, the network adjusts the values of its internal pathways, or weights. If training is successful, the internal parameters are then adjusted so that the network can produce correct classifications in response to similar new input data patterns.

Once trained, a network's response can be insensitive to minor variations in its input. This ability to see through noise and distortion to the inherent pattern is vital to pattern recognition in a real work environment. Usually, sets of training examples are presented repeatedly during training to allow the network to adjust its internal parameters gradually. The computer software responds to each example by randomly activating its circuits in a particular configuration. Any connections that produce a correct answer are reinforced, and connections that produce an incorrect answer are weakened. After several thousand trials, the network activates only those circuits that produce the correct answer. Neural networks can be divided into several categories based on the method of learning, structure, and control. The most basic division among networks is the direction that information flows. In feed-forward networks, PEs receive input only from the previous layer's input. Network learning can also be supervised or unsupervised. For supervised, an associated output response is supplied to the network for each input pattern during the learning phase. For unsupervised, the network clusters the input data into appropriate groups.

Artificial neural networks consist of three key components: PEs, layers, and connections (Figure 5-22). Artificial neural networks borrow the basic operating procedures of their biological counterparts, the mammalian brain. A "neuron" or PE in a first layer receives some external stimulation that is weighted and passed through to the next layer of PEs. In our implementation of neural networks, each PE received one element of the input pattern (i.e., one elemental assay from one sample). The signals coming into the PEs in the second layer were multiplied by the connection weights between the two layers and summed over all the connection weights. This sum is commonly referred to as the activation of the PE. If the activation exceeds the threshold of the PE, it fires. Once fired, the activation is modified by a transfer function and sent to the next layer of PEs. The exact form of the summation processes, transfer functions, and interconnection scheme varies according to the type of network. 


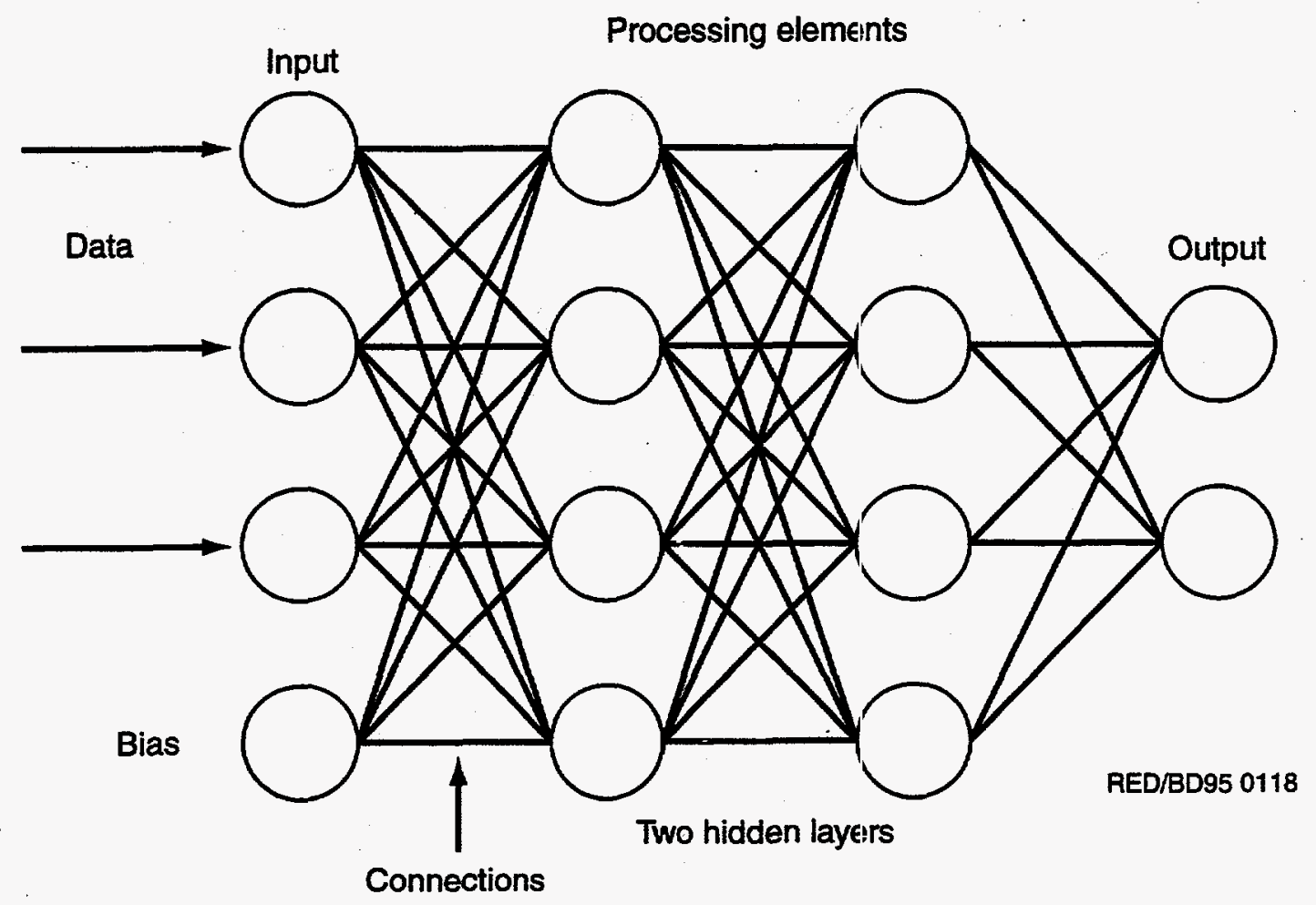

Figure 5-22. A feed-forward, three layer neural network for supervised learning.

The memory of an artificial neural network system is the values of the connection weights between PEs, that is, how they process new data in the context of the learning set (Caudill 1988, Rumelhart et al. 1986, Anderson and Rosenfeld 1989, Dayhoff 1989, Wasserman 1989, Freeman and Skapura 1991).

\subsubsection{Classification Task Paradigms}

Two basic paradigms for the classification tasks were used in this work. These paradigms, backpropagation and radial basis functions (RBFs), are described below.

5.6.2.1. Backpropagation. Backpropagation is a type of nonlinear regression. The technique seeks a function that maps a set of input patterns to a set of output patterns with a minimum error. Derived independently by Werbos (1974) and Parker (1989) and popularized by Rumelhart et al. (1986), backpropagation is the most popular and best understood neural paradigm. It has, however, virtually no biological fidelity.

Figure 5-22 shows a three-layer backpropagation network. The input pattern $X_{i p}$ is multiplied by the connection weighs $\mathrm{W}_{\mathrm{i}, \mathrm{j}}$, leading to the hidden layer so that the sum at a given hidden-layer PE is

$$
S_{j p}=\sum_{i} X_{i p} W_{i j}+\theta_{j}
$$

Theta, $\theta$, is a bias term with a fixed value of 1.0 but with trainable connection weights. The sum $\mathrm{S}_{\mathrm{j}}$ is modulated by a threshold function, $\mathrm{f}(\mathrm{S})$, that is normally sigmoidal in shape. This shape is similar 
to the thresholding performed by biological neurons. Common thresholds are the logistic function and hyperbolic tangent given respectively as

$$
\begin{aligned}
& f(S)=\frac{1}{1+e^{-S_{\jmath}}} \\
& f(S)=\frac{e^{S_{\jmath}}-e^{-S_{\jmath}}}{e^{S_{j}}+e^{-S_{j}}}
\end{aligned}
$$

The derivative of the sigmoid function reaches a maximum around 0.5 , so the weight change allowed by the sigmoid is maximum for PEs in their midrange. In other words, PEs that are not committed to being on or off are allowed to change most rapidly. The activation of a PE is

$$
A_{j p}=f_{j}\left(S_{j p}\right)=\frac{1}{1+e^{-s_{j p}}}
$$

The bias term in Equation (5-12) shifts the transfer function laterally, allowing each PE to have an optimal threshold function. A gain term can also be applied to change the slope of the threshold. The activation of the hidden PE becomes the input to the next layer of PEs.

$$
\begin{aligned}
& X_{k p}=A_{j p} \\
& S_{k p}=\sum_{i} X_{j p} W_{j k}+\theta_{k} \\
& A_{k p}=f_{k}\left(S_{k p}\right)=\frac{1}{1+e^{-S_{k p}}} \\
& E_{k p}=\left(D_{k p}-A_{k p}\right)
\end{aligned}
$$

The activation $A_{k p}$ is compared to the desired output pattern $D_{k p}$ to obtain an error $E_{k p}$. This error must be apportioned to all the connection weights in the network through the back-error propagation cycle. The delta weights between the layers are computed as 


$$
\begin{aligned}
& \delta_{k p}^{o}=\left(D_{k p}-A_{k p}\right) f_{k}^{\prime}\left(A_{k p}\right) \\
& \delta_{j p}^{h}=f_{j}^{\prime}\left(A_{j p}\right) \sum_{k} \delta_{k p}^{o} W_{j k}
\end{aligned}
$$

where $f^{\prime}$ is the derivative of the threshold function. For the logistic function, we use $f^{\prime}(S)=f(S)$ $(1-F(S))$, and for the hyperbolic tangent the equation becomes $f^{\prime}(S)=(1+f(S))(1-f(S))$.

Since the error for the hidden PEs is unknown, we must use the sum of the delta weight $\delta_{k p}^{\circ}$ times the connection weights from the output layer as the error. Weights are updated as

$$
\begin{aligned}
& W_{j k}^{\prime}=W_{j k}+\eta \delta_{k p}^{o} A_{j p}+\alpha^{o} \Delta W_{j k} \\
& W_{i j}^{\prime}=W_{i j}+\eta \delta_{j p}^{h} X_{i p}+\alpha^{h} \Delta W_{i j}
\end{aligned}
$$

where $\eta$ is a step size or learning rate set between 0 and $1 ; \alpha$ is a nomentum term set between 0 and 1 ; and the delta weight is the weight change from the previous iteration. The addition of the momentum term helps move the network out of some local minima in error spare. The use of momentum in a neural network is analogous to using a conjugate gradient technique. Equations (5-21) and (5-22) are known as the generalized delta rule.

After the weights are updated, one iteration is complete, and the entire process is repeated until an acceptably small error $E_{p}$,

$$
E_{p}=\frac{1}{2} \sum_{k=1}^{m} \delta_{k p}^{2 o}
$$

is achieved for each pattern. Weight updates are made after each pattern is presented (i.e., interaction) or accumulated over the entire training set (i.e., epoch) or some subset thereof.

5.6.2.2. Radial Basis Function. RBFs as described by Moody and Darken (1989), Poggio and Girosi (1990), and Musavi et al. (1992) are a class of networks that approximate functions by using linear combinations of radially symmetric functions. The network consists of one hidden layer in which each PE evaluates a kernel function on the incoming signal and an output layer that computes a weighted linear sum of the kernel functions. The kernel function has maximal response when the input is closest to its center and decreases monotonically as the Euclidean distance from the center increases. The kernel function is usually Gaussian. Training consists of finding centers, widths, and weights connecting hidden nodes to output nodes. While we could place a Gaussian function on every training pattern and train the kernel to peak when the pattern is presented, it is not a practical approach for large numbers of training 
exemplars. The initial phase of training is unsupervised. A K-means clustering algorithm is used to find the centers of the kernels in a manner similar to Kohonen (1988) competitive learning. A nearest neighbor approach is used to find the width of the kernel. We start with a random set of centers $C_{k}$ and calculate the distance from each training pattern to each center. The closest center is modified as

$$
\begin{aligned}
& C_{k}^{\prime}=C_{k}+\eta\left(X_{i j}-C_{k}\right) \\
& \sigma_{k}=\sqrt{\frac{1}{P} \sum_{p=1}^{p}\left\|C_{k}-C_{k p}\right\|^{2}}
\end{aligned}
$$

The width of the kernel function is given as the distance from the cluster center to the P nearest neighbor cluster center.

Gradient descent can then be used to adjust the weights between the hidden and output layers as in backpropagation. We have to specify how many Gaussians we want to use along with all the parameters for the backpropagation algorithm.

Leonard and Kramer (1991) show a comparison of the decision functions formed by RBFs and backpropagation networks and suggest that radial basis functions networks are able to form decision regions further from the training data, are able to form bounded decisions regions, and are generally more accurate for incomplete or unrepresentative data.

\subsubsection{Method}

5.6.3.1 Target Versus Background. We divided the target versus background classification task into several progressively harder subtasks. The first subtask was to train networks specifically for each experiment, each level, and each sensor individually. Such an approach requires many networks but allows one to see how well the networks can learn specific data. The second task involved combining sensors within a level for each experiment. Our third task involved combining the levels of each experiment for each sensor and for combined sensors. Our fourth task involved combining data from several experiments (we only worked with Experiments 2, 3, 5, and 6).

5.6.3.1.1. Creating Training and Testing Files-Training files were extracted from the raw data by writing every third value of the raw data file to the training file. The raw data were not gridded or processed in any way before creating the training file. The subsampling was done to produce a minimum training file while leaving some data available for testing the network. No attempt was made to compensate for the fact that subsampling might miss some small targets, would produce little data within some targets, or in general might not lead to an optimum training set. We felt that this training set would produce our most conservative results. 
A training file consisted of an input vector and output vector. The input vector contained the raw data from the sensors; the output vector was the desired class such as target or background. In some cases, our input vector contained the $x, y, z$ values for the data point, but these values were never used for any calculations in the neural network. To create a training vector for magnetic data only, sensor 1 data were written to the training file. To create a training vector for EM data only, data from sensors 2 (horizontal quadrature), 3 (vertical quadrature), 4 (horizontal in-phase), and 5 (vertical in-phase), in that order, were written to a training file. When magnetic and EM data were combined, they are written as sensors 1 through 5 from left to right across the input vector. Table 5-10 lists the sensor designations.

To create an output vector we needed to know if the $\mathrm{x}, \mathrm{y}$ coordinates for any given data point were located within the boundaries of known targets. Slightly different approaches were taken for the EM and magnetic data. For the EM data, our extraction program read four $x, y$ corner-coordinates for each target and checked the data point to see if it fell within those bounds. Round targets were extracted as squares so some inaccuracies existed around their edges. Generally, the neural networks were able to recognize the data points that we said were target but were in fact background around the circular targets. The output was written as -1 if the data point was background and +1 if the data point was within a target. When training only on magnetic data, we had to compensate for the fact that the magnetic anomaly did not always overlie the target. Thus, we determined the output criteria of target or background directly from the magnetic data. We averaged all of the magnetic values for a training file and took all values above the average as target and values at or below the average as background. The network results were greatly improved for magnetic classification after performing this thresholding for experiments one and two but little improvement was seen for Experiments 5 and 6 . When EM and magnetic data were combined, the magnetic data were not preprocessed, thus our initial preprocessing did not affect the final results. Test sets consisted of the entire data set, so testing included training data and new data. The column containing the desired output class was sometimes appended to the test sets for reference purposes only and was ignored by the networks.

5.6.3.1.2 Designing and Training Networks-For the first task, a backpropagation network was designed with an input vector equal to the size of the training vector (e.g., four input PEs for EM, two for magnetics). The size of the hidden layer was determined experimentally and was generally set as one PE less than the input vector. The output vector consisted of one PE. We used a hyperbolic tangent for our threshold function. We used the generalized delta rule with momentum for our learning rule. Weights were updated after each input pattern was presented. Training progressed until the root mean square error stabilized at an acceptably low level. Typically training was continued for 20,000 iterations. An iteration is one complete pass, forward and backward, through the network for one training pattern. Training times on a $90 \mathrm{MHz}$ Pentium computer for 20,000 iterations were typically 30 seconds. - Testing a complete data set for one level, one sensor (989 to 1,075 points) took approximately 2 seconds. All training and testing times include the time it takes to refresh the screen graphics.

When backpropagation failed to produce an accurate map of the targets, we used a RBF network. An RBF network contains the same input vector as backpropagation but has an extra layer that contains Gaussian kernel functions that perform an initial clustering of the input vectors. Some of these networks also contained a hidden layer with three PEs and a single output PE. Weights were updated after every pattern was presented. Training times were 50,000 iterations in 2 minutes. Testing a complete data set for one level, one sensor ( 989 to 1,075 points) took approximately 3 seconds. 
Table 5-10. Digface characterization sensor description.

\begin{tabular}{cl}
\hline Sensor & \multicolumn{1}{c}{ Description } \\
\hline 1 & Magnetic field, vertical component and vertical component gradient \\
2 & Ground electromagnetic (EM) conductivity, horizontal dipole, quadrature phase \\
3 & Ground EM conductivity, horizontal dipole, inphase \\
4 & Ground EM conductivity, vertical dipole, quadrature phase \\
5 & Ground EM conductivity, vertical dipole, inphase \\
6 & Volatile organic compound sensor \\
\hline
\end{tabular}

The second task involved combining sensors within a level for each experiment. This provided some improvement in the results, especially when objects with strong magnetic signatures were involved. We typically used RBF networks with six input, zero to four hidden PEs, and one output for this process. However, at times no hidden PEs were required. Training times for 50,000 to 100,000 iterations were from 2 to 4 minutes. Testing a complete data set of 989 points still took only 3 to 4 seconds.

The third task involved combining the levels of each experiment for each sensor and for combined sensors. This provided a more dramatic improvement in the results. Targets that were previously located as blobs were sharpened to the point that they were easily recognizable. Both the EM-combined level results and the EM/magnetic-combined level results cleanly find all of the targets that remained throughout the entire experiment (some targets were removed during excavations). Backpropagation networks with two hidden layers were found to give the best results for this task. Input vector sizes ranged from 12 to 44 with the first hidden layer having one less PE than the input layer and the second hidden layer having half as many PEs as the first. An average training time was about 4 minutes for 70,000 iterations. Testing a 989 point data set required only about 7 seconds. We attempted intertesting the experiments at this point, but had limited success.

The fourth task involved combining data from several experiments (again, working with Experiments 2, 3, 5, and 6) into the same training set. We tried different combinations of targets to find the training set that would produce the best test results on all experiments. We found to get good test results we needed to include targets from each experiment. The targets included in the final training set were objects $6,7,9,10,17,23,25,27,28,29$, and 34 . The test results on this cut-and-paste training set were not as sharp for Experiments 5 and 6 as those from the third task, but the results for Experiments 2 and 3 were comparable. We used both magnetic and EM data and accumulated the scan-level data (i.e., a net for only level 0 contained data from only level 0 whereas a net for level 5 contained data from levels 0 through 5). All training times and number of required training iterations were greatly enlarged for this task, but the testing times remained around 7 seconds.

5.6.3.2 Depth Estimates. The $\mathrm{x}, \mathrm{y}$ locations previously classified as targets were used to make depth estimations. We trained several networks to estimate depth to targets. We started with only the targets in Experiment 2 to determine if a network could estimate depth with point data from EM and magnetic sensors. A training file consisted of an input pattern containing information from both sensors 
for every other data point in one level and output of the depth to the target at that level. The network successfully estimated depths within $5 \%$ for the two targets in Experiment 2 . Next, we trained a network using only the metallic targets from Experiment 5 excluding targets 18 and 19. Again the depth estimates were good. We trained another network on only the boxes in Experiment 5. Then we combined the metallic and nonmetallic targets into the same training set. For Experiment 6, we trained on the targets that the classification network easily identified (targets 25, 26, and 28). The depth estimates for these targets were good. Next we trained on the hard targets, the bars, pipes, and C-channel. We used only those data points the classification network identified as target for training. While the results were not within our desired accuracy range for this network, the minimum depth estimated for a target was conservative enough that the operator of an excavator would never encounter a target at a depth shallower than the network estimated. For the Experiment 6 depth network, we used the data points within the actual target boundaries for training.

Finally, we combined targets from Experiments 2, 5, and 6. Llata from Experiment 3 were not used for training because the objects were stacked. Objects $6,7,15,16,17,20,21,23,24,25,26$, and 28 were included in the training set. Both magnetic and EM data were used as the input vector. We used the data that the target versus background networks had called target for training purposes. Hence, objects that were poorly classified may have larger depth errors. The output PE is the depth to the top of the target from the given level. The training set consisted of every $\mathrm{x}, \mathrm{y}$ coordinate within the objects for every level of data, so there were up to six or seven different depths represented for each object in the training set. All objects from Experiments 2, 3, 5, and 6 were run throughout the trained network for testing.

The training time for the final depth estimation network was approximately 3 minutes for 100,000 iterations. The training set contained 1,727 data points. Testing targets from the individual experiments required only about 2 seconds per experiment including all levels.

5.6.3.3 Conductivity Estimates. We used essentially the same training files for the conductivity classification as for the depth estimation. Our goal was to classify the previously identified targets as either conductive or resistive. Since depth of burial affects the magnitude of the anomaly, we included depth as a second classification parameter. Thus, we had four desired classes: deep $(-1)$ conductive $(+1)$; shallow $(+1)$ conductive; deep resistive $(-1)$; and shallow resistive. We used both EM and magnetic data for input although the magnetic data probably did not contribute significantly to the results. More should be done with this classification to further refine it. We did not establish that including depth as a class is mandatory nor did we determine if we could perform a finer classification and include moderately conductive or moderately resistive classes.

\subsubsection{Results}

Spreadsheets and grids generated from output can be used to portray results of the neural network study. Only a few output grids from Experiment 2 are presented here. Spreadsheets will be used to summarize results of the work to date. Target criteria grids and $C$ language source code for one of the neural networks are given in Appendix C.

5.6.4.1 Target Location. As described above, task one involved training networks on data from single levels and for a sensor. Figures 5-23 and 5-24 show gridded output from networks trained specifically for magnetic data from Experiment 2 levels 2 and 5. In these figures, the light areas indicate 
$\nabla L-\mathcal{S}$

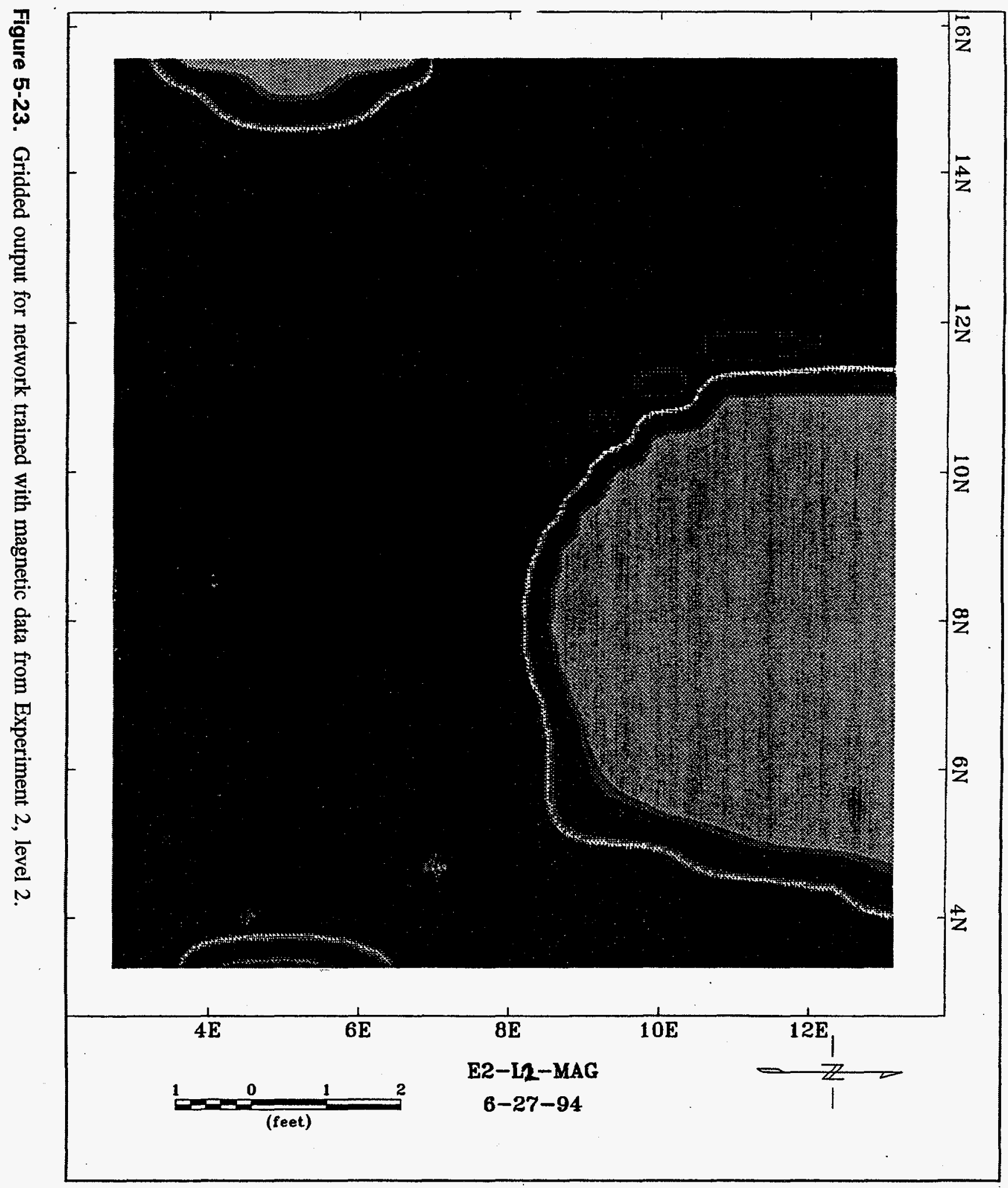




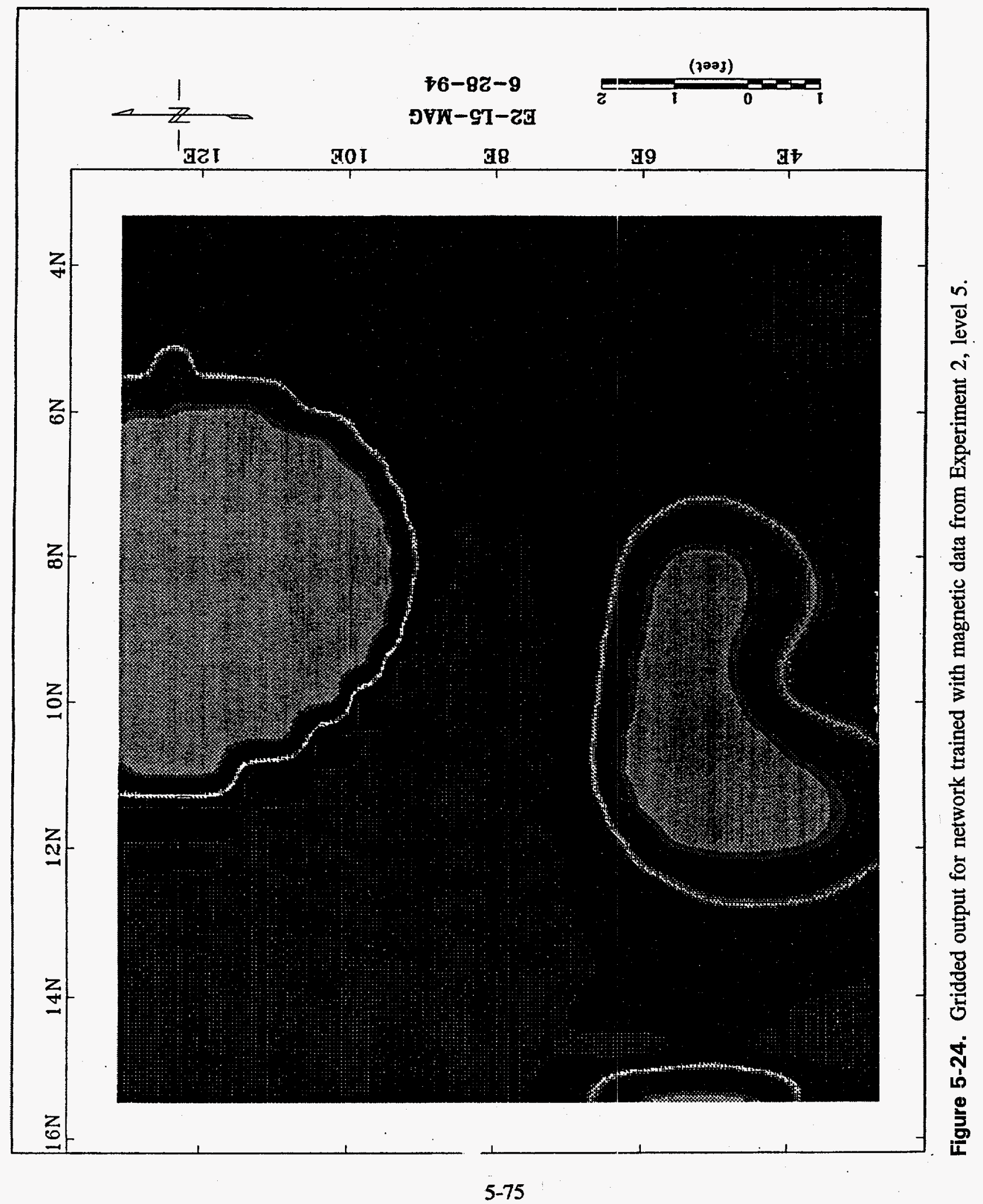


target and the dark areas indicate background. There were two targets in Experiment 2 (see Appendix A) a 55-gal barrel containing concrete on the right-hand side of Figures 5-23 and 5-24 and a wooden box containing nonferrous metals on the left-hand side of the figures. The arrow labeled $\mathrm{N}$ on the gridded output represents the top of the target location pages shown in Appendix A, not true north. From these figures, it is apparent that at level 2, the network identified the metallic target but not the wooden box. This is not surprising because the box and contents were essentially nonferrous although there were metal bands around the box. At level 5, however, the network identified both targets suggesting that the improved resolution in the data (the magnetic sensor was closer to the target) played a key role. Improvement in data resolution as the sensors move closer to the target is one of the key themes of the digface approach and seems to carry over to the network application. Figures 5-25 and 5-26 show gridded output from networks trained on EM data for the same levels and experiment. Figure 5-25 shows that at level 2, unlike the networks trained on magnetic data alone, the EM-trained network located both targets. So both the targets in Experiment 2 are significant perturbations to the local conductivity so there was a respectable EM signature at level 2. Figure 5-26 shows that at level 5, the EM-trained network output was not significantly different relative to level 2 . In fact the EM-trained network located the targets at all seven levels in this experiment although the signature was somewhat weak at levels 0 and 1.

The results described above for networks trained on the various levels for Experiment 2 are reasonably typical for the other experiments analyzed. In general, increasing resolution in the data (sensors moving closer to the target), resulted in improved target location by the networks.

In task 2, networks were trained within a level using both the magnetic and EM sensor data. Figures 5-27 and 5-28 show gridded output for level 0 and level 6 in Experiment 2. As shown in Figure 5-27, the network located the targets even at level 0 . This performance is better than when using either the magnetic or EM input alone (see Figures 5-23 and 5-24).

Figure 5-29 shows the results for the magnetometer sensor trained on combined level information from previous levels when applied to data from level 6 in Experiment 2. Both targets are clearly identified and the target shapes are relatively good. Combining level information in this manner improved results for situations where some of the targets had a strong magnetic signature.

Figures 5-30 and 5-31 show the output from networks trained using the EM data from all levels in Experiment 2 and the combined magnetic and EM data from all levels in Experiment 2, respectively. The combined previous level information is used in training and then applied to data from Experiment 2, level 6 for the results shown in the figures. In both cases, the targets are clearly identified. Notice that the EM-trained network produced output that resulted in target shapes that are somewhat sharper and more realistic relative to the magnetic sensor-trained network. Figure 5-31 shows the result of combining both the EM and magnetic data and using all measurement levels in the training. The output is similar to that in Figure 5-30. In general, the networks trained using the combined levels of EM data and the combined levels of EM and magnetic data were able to find all the targets that remained throughout the experiment (in some experiments, targets were removed during overburden excavation).

Table 5-11 summarizes the discussion presented above for all of the experiments. This table the results of tasks 1,2 , and 3 and describes the results for the different trials conducted for testing on the same experiment used for training. The columns correspond to trials using magnetics, EM, and both sensors for each experiment. The rows correspond to each scan level. For each trial, the network 
LLSS

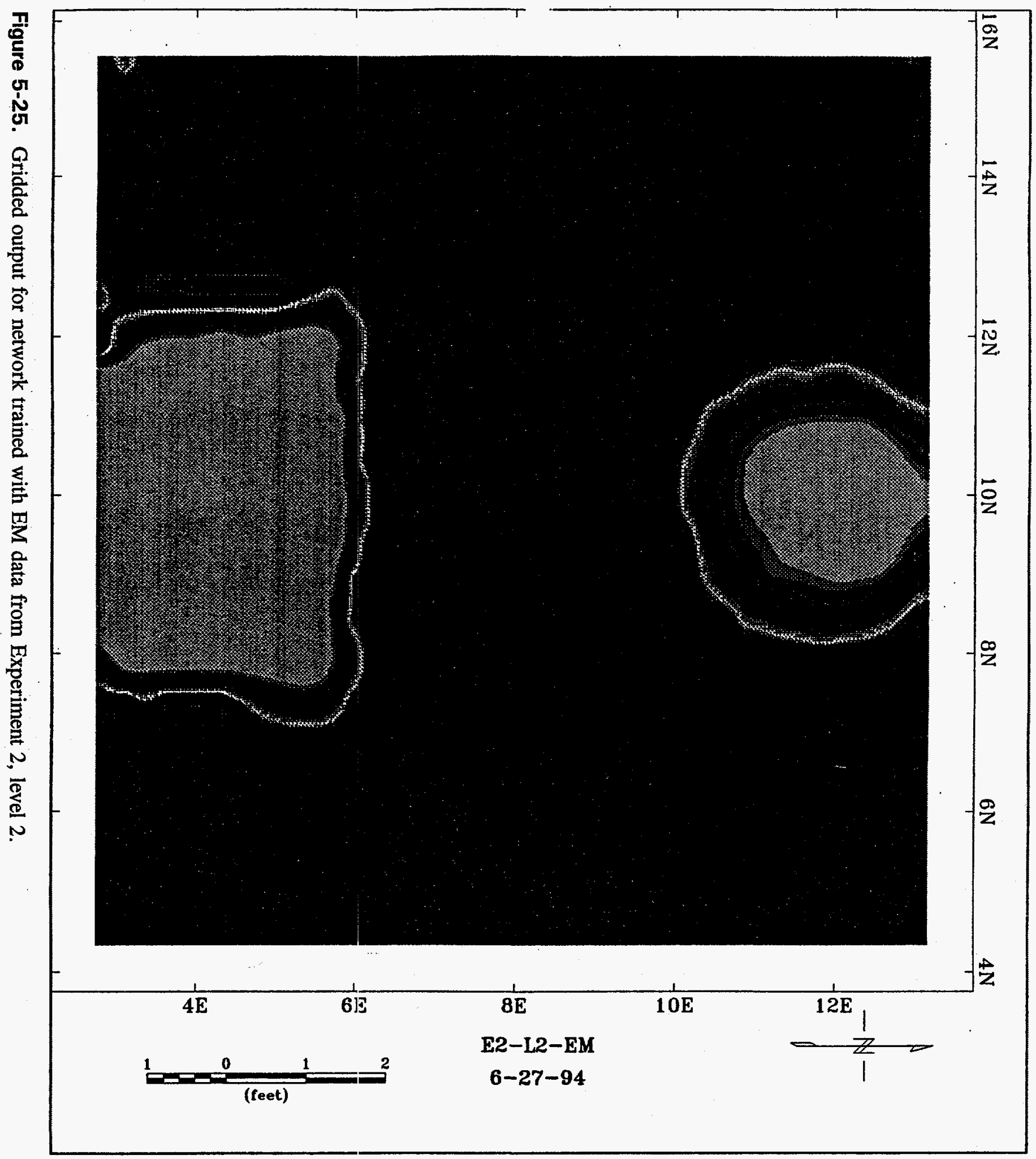




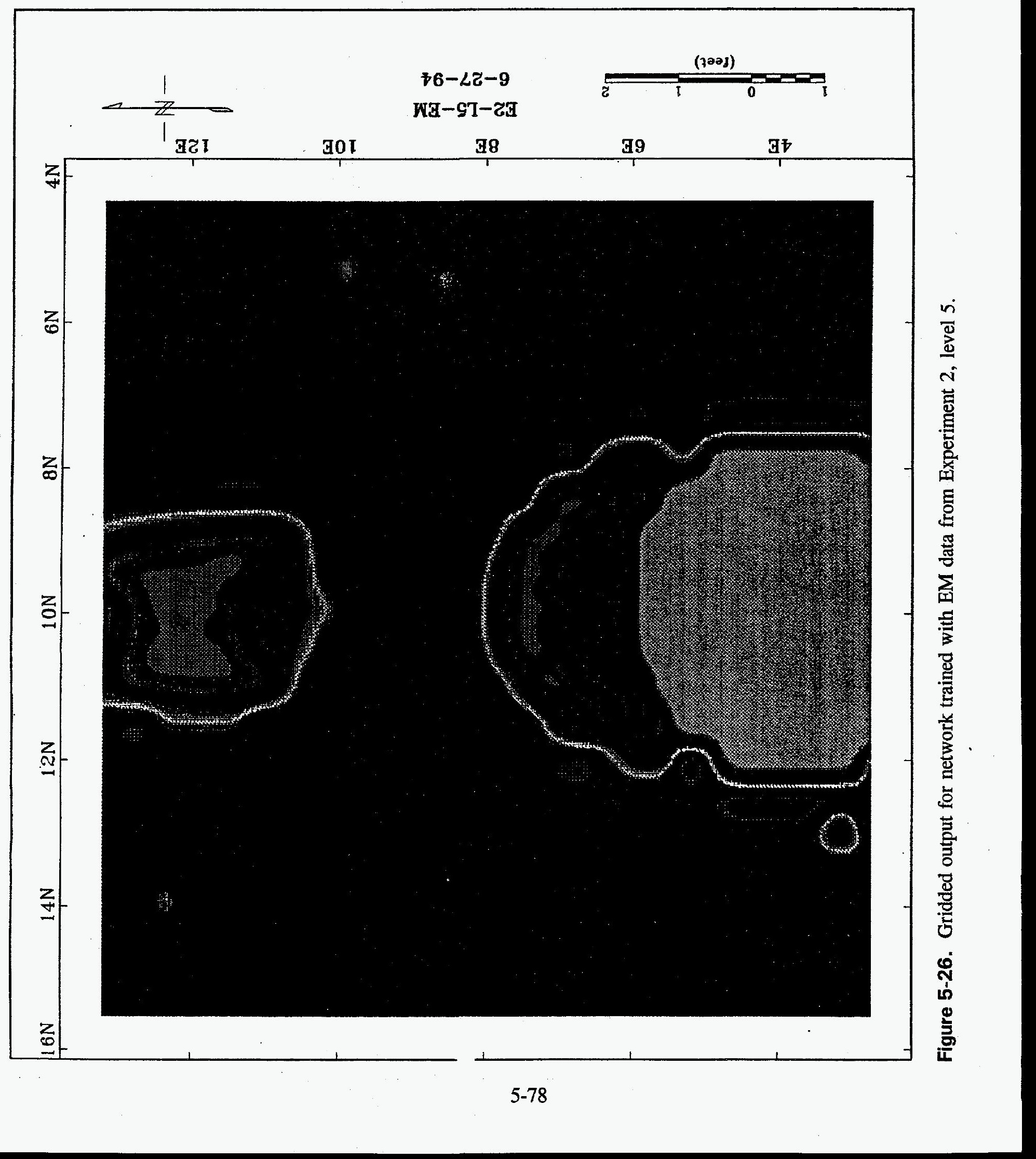


$6 L-S$

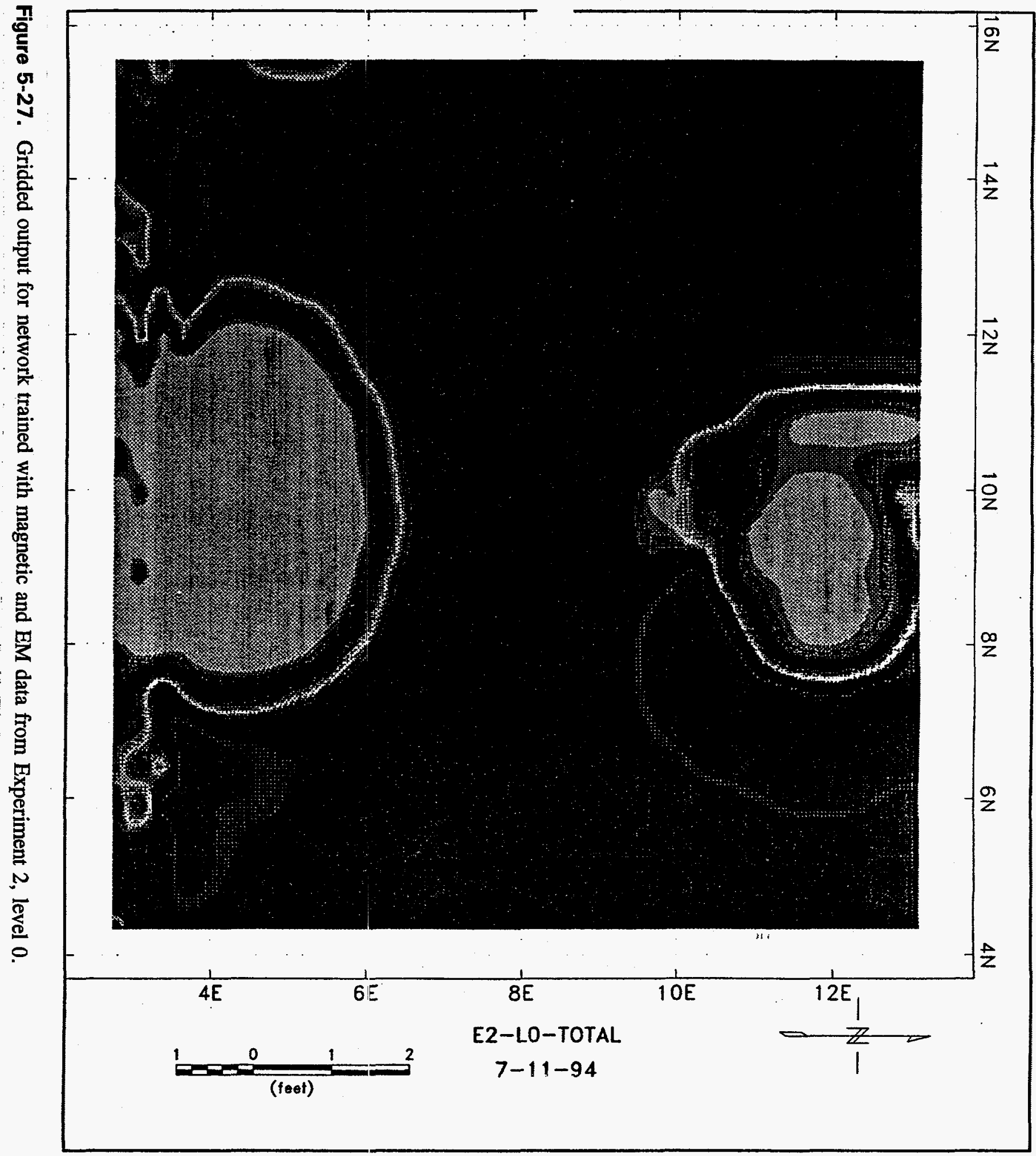




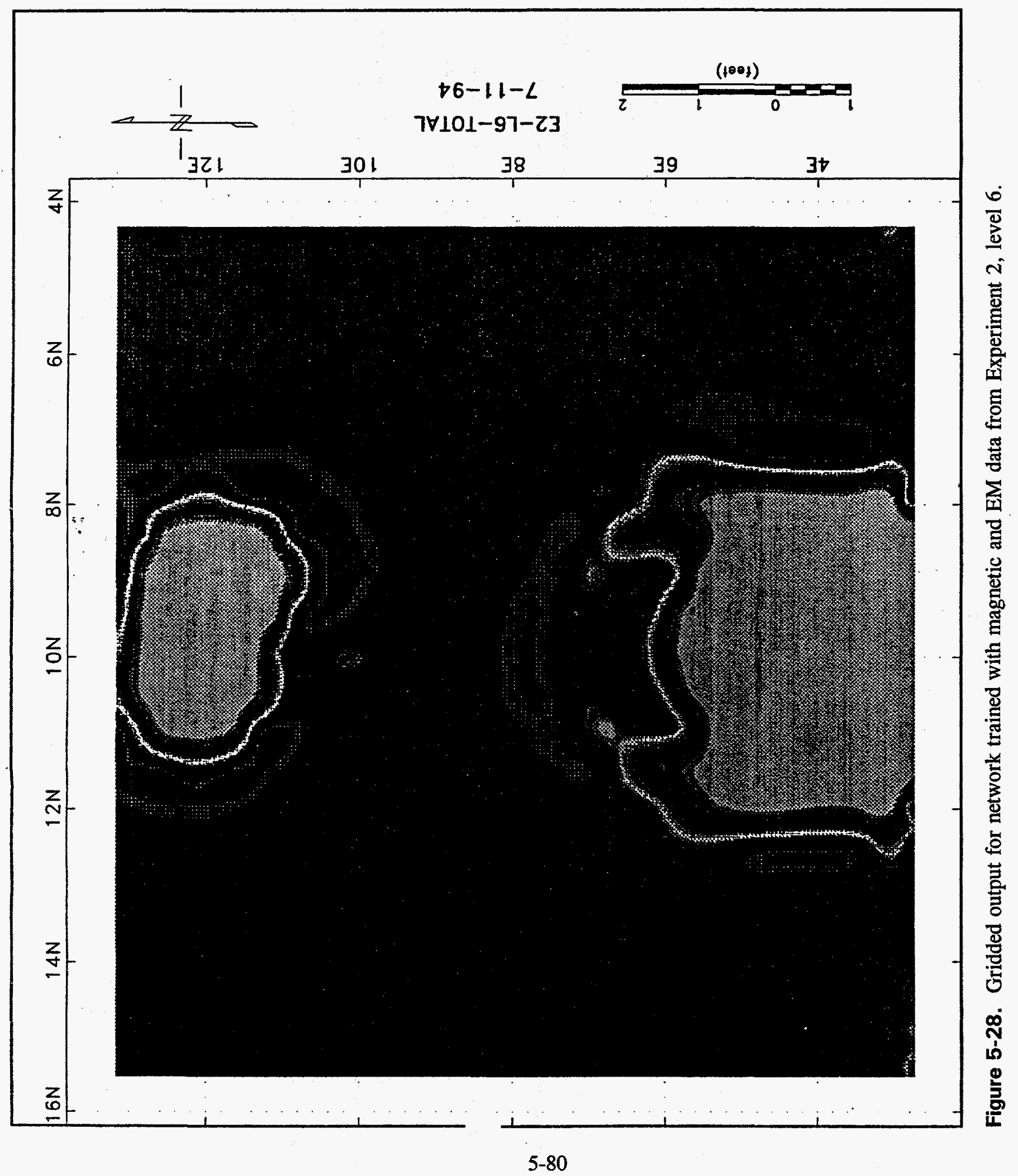




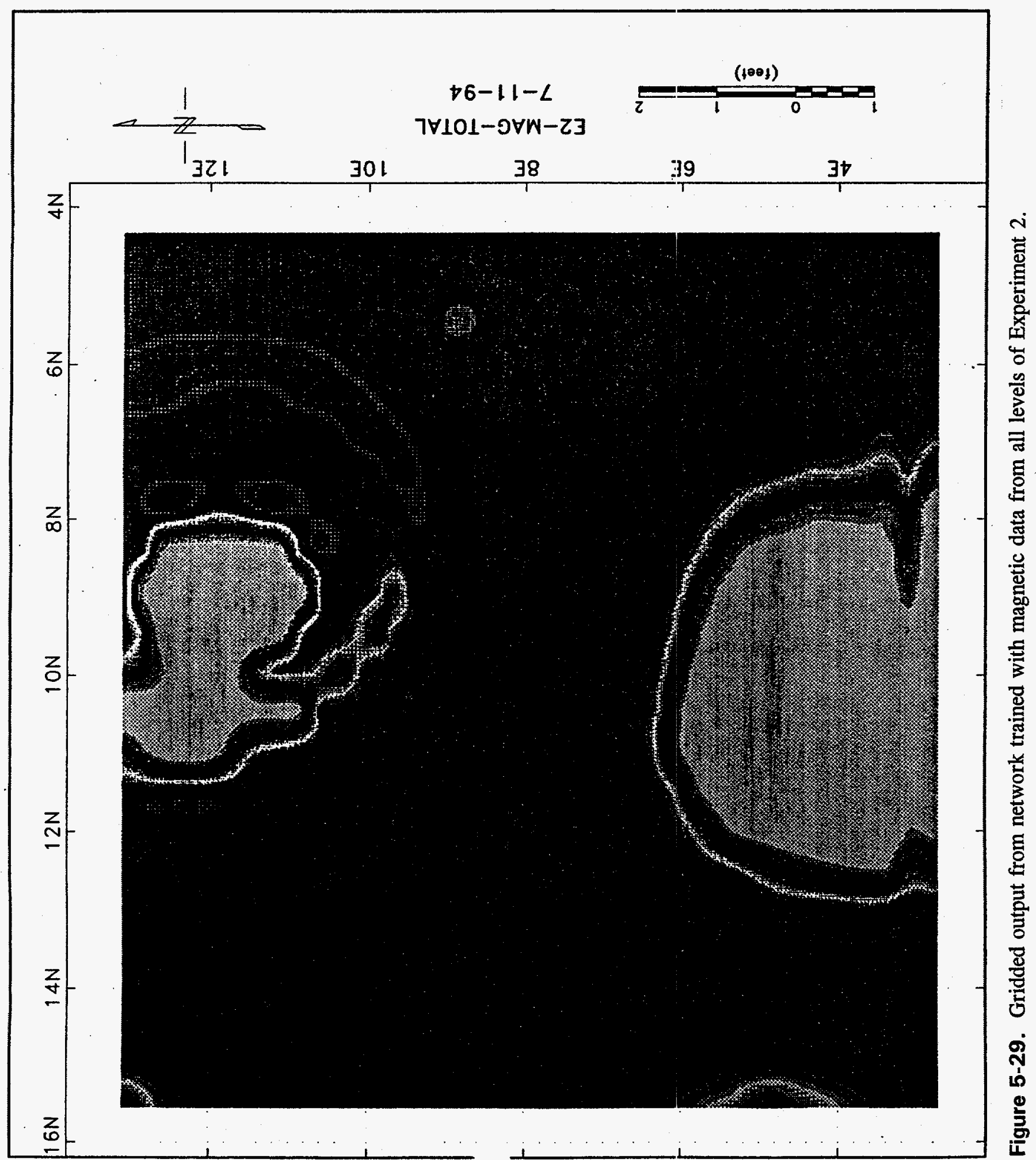




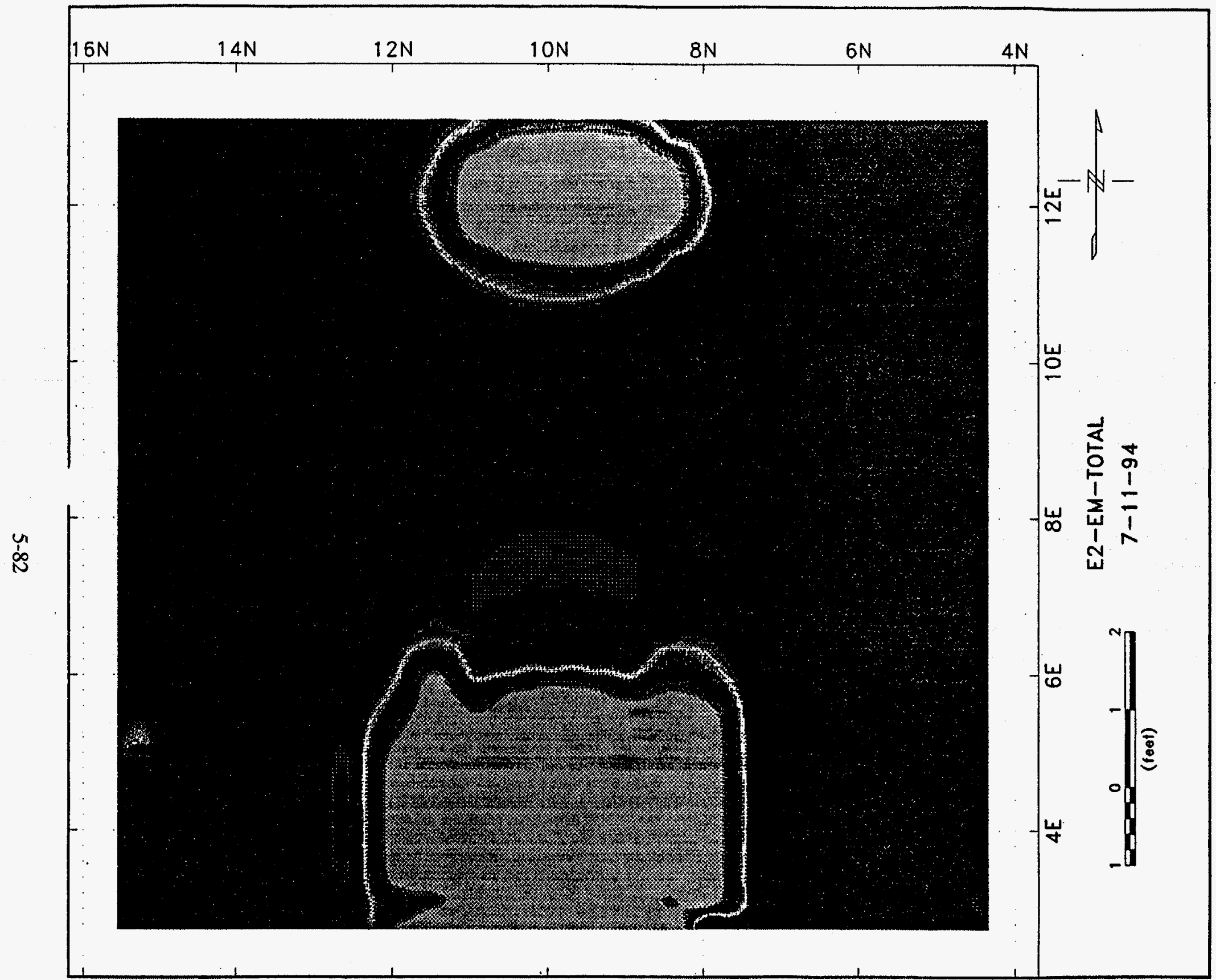

Figure 5-30. Gridded output from network trained with EM data from Experiment 2. 


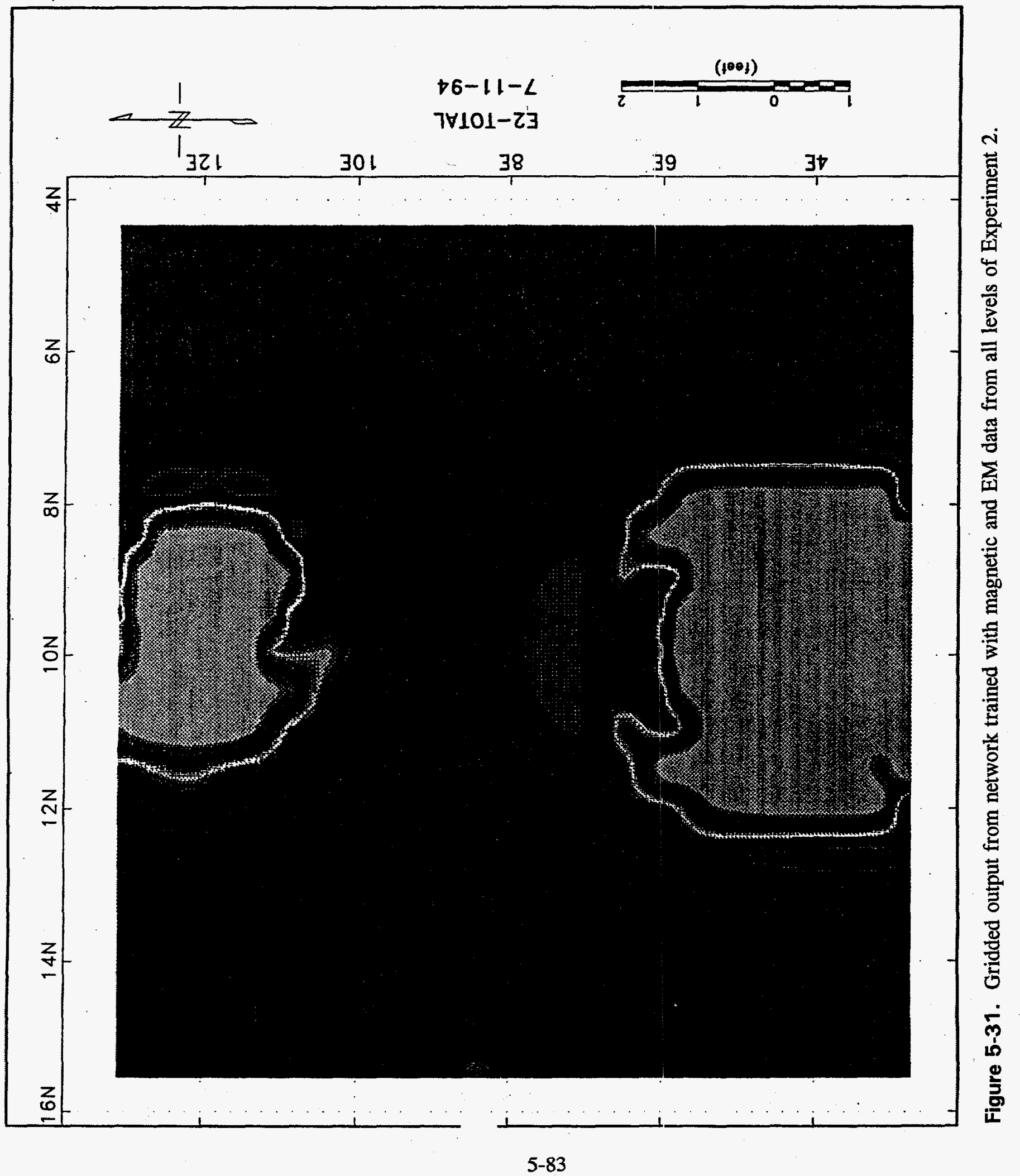


Table 5-11. Summary of neural network training and testing results.

\begin{tabular}{|c|c|c|c|c|c|c|c|c|c|c|c|c|c|c|c|c|c|c|c|}
\hline & & & Ex.\#1 & & & Ex, \#2 & & & $E x, \# 3$ & & & Ex.\#4 & & & Ex. $\# 5$ & & & Ex. \#6 & \\
\hline & & Mag. & JeM & Total & Mag. & IEM & Total & Mag. & TEM & Total & Chem. & TEM & Total & Mag. & EM & Total & Mag. & TEM & Total \\
\hline Level \#0 & $\begin{array}{l}\text { Net Typo } \\
O G, C R\end{array}$ & \begin{tabular}{|l}
$B P(2,1,0)$ \\
$85, .87$
\end{tabular} & $\begin{array}{l}\mathrm{AB}(2,50,0) \\
66, .74\end{array}$ & & $\begin{array}{l}\mathrm{BP}(2,1,0) \\
.98,99\end{array}$ & $\begin{array}{l}\mathrm{BP}(7,4,0) \\
.81,81\end{array}$ & $\begin{array}{l}\mathrm{RB}(6,50,0) \\
.91, .96\end{array}$ & $\begin{array}{l}\mathrm{BP}(2,1,0) \\
.98,1.0\end{array}$ & $\begin{array}{l}\mathrm{BP}(7,6,0) \\
.95, .94\end{array}$ & $\begin{array}{l}\mathrm{AB}(6,50,0) \\
.94, .98\end{array}$ & & & & $\begin{array}{l}\mathrm{BP}(2,1,0) \\
99,1.0\end{array}$ & \begin{tabular}{|l|}
$\mathrm{RB}(7,50,0)$ \\
$63, .81$
\end{tabular} & $\begin{array}{l}R B(6,50,0) \\
.66, .82\end{array}$ & $\begin{array}{l}B P(2,1,0) \\
.97, .99\end{array}$ & $\begin{array}{l}\mathrm{RB}(7,50,0) \\
53, .70\end{array}$ & $\begin{array}{l}\mathrm{AB}(6,50,0) \\
.51, .67\end{array}$ \\
\hline Level \#1 & $\begin{array}{l}\text { Net Typo } \\
O C, C R \\
\end{array}$ & $\begin{array}{l}\mathrm{BP}(2,1,0) \\
88, .88\end{array}$ & $\begin{array}{l}\mathrm{AB}(2,50,0) \\
66, .74\end{array}$ & & $\begin{array}{l}\mathrm{BP}(2,1,0) \\
.98, .99 \\
\end{array}$ & $\begin{array}{l}\mathrm{BP}(7,4,0) \\
.89, .93\end{array}$ & $\begin{array}{l}\mathrm{RB}(6,50,0) \\
.92, .94\end{array}$ & $\begin{array}{l}\mathrm{BP}(2,1,0) \\
.97, .98 \\
\end{array}$ & $\begin{array}{l}\mathrm{BP}(7,6,0) \\
.94,94 \\
\end{array}$ & $\begin{array}{l}\mathrm{AB}(6,50,0) \\
.97, .99\end{array}$ & & & & $\begin{array}{l}\mathrm{BP}(2,1,0) \\
.94, .93\end{array}$ & $\begin{array}{l}\mathrm{AB}, \\
\mathrm{RB}(7,50,0)\end{array}$ & $\mid \begin{array}{l}\mathrm{RB}(6, .5 \mathrm{~L} \\
.67, .82\end{array}$ & $\begin{array}{l}\mathrm{BP}(2,1,0) \\
.96,1.0\end{array}$ & $\mid \begin{array}{l}\mid .50, .10 \\
8 B(7,50,0) \\
.52, .67\end{array}$ & 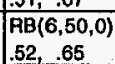 \\
\hline Level H2. & $\begin{array}{l}\text { Net Type } \\
0, \text { CR }\end{array}$ & $\begin{array}{l}B P(2,1,0) \\
.95, .94 \\
\end{array}$ & $\begin{array}{l}\mathrm{AB}(2,50,0) \\
63, .73 \\
\end{array}$ & & $\begin{array}{l}\mathrm{PP}(2,1,0) \\
.97, .98 \\
\end{array}$ & $\begin{array}{l}B P(7,4,0) \\
.93,96 \\
\end{array}$ & $\begin{array}{l}\text { RB }(6,50,0) \\
.93, .95 \\
\end{array}$ & $\begin{array}{l}\mathrm{BP}(2,1,0) \\
.96, .97 \\
\end{array}$ & $\begin{array}{l}B P(7,6,0) \\
.93,95 \\
\end{array}$ & $\begin{array}{l}R B(6,50,0) \\
.97, .99\end{array}$ & & & & \begin{tabular}{|l|}
$\mathrm{BP}(2,1,0)$ \\
$98, .99$
\end{tabular} & \begin{tabular}{|l|}
$\mathrm{RB}(7,50,0)$ \\
$.59, .76$ \\
\end{tabular} & $\begin{array}{l}\mathrm{RB}(6,50,0) \\
.66, .80\end{array}$ & $\begin{array}{l}\mathrm{BP}(2,1,0) \\
.95, .98\end{array}$ & $\begin{array}{l}\mathrm{hB}(7,50,0) \\
49, .66\end{array}$ & $\begin{array}{l}\mathrm{RB}(6,50,0) \\
.48, .67\end{array}$ \\
\hline Level \#3 & $\begin{array}{l}\text { Net Type } \\
\text { OG,CA }\end{array}$ & $\begin{array}{l}B P(2,1,0) \\
.92, .93 \\
\end{array}$ & $\begin{array}{l}\mathrm{FB}(2,50,0) \\
.57, .66\end{array}$ & & $\begin{array}{l}\mathrm{BP}(2,1,0) \\
.96, .98 \\
\end{array}$ & $\begin{array}{l}\mathrm{BP}(7,4,0) \\
.91,95 \\
\end{array}$ & $\begin{array}{l}\mathrm{AB}(6,50,0) \\
.93, .95\end{array}$ & $\begin{array}{l}\mathrm{BP}(2,1,0) \\
.95, .96\end{array}$ & $\begin{array}{l}\mathrm{BP}(7,6,0) \\
95, .97\end{array}$ & $\begin{array}{l}\mathrm{AB}(6,50,0) \\
.97, .99\end{array}$ & & & & $\begin{array}{l}\mathrm{BP}(2,1,0) \\
98,98\end{array}$ & $\begin{array}{l}\mathrm{RB}(7,50,0) \\
.56, .70\end{array}$ & $\begin{array}{l}\mathrm{RB}(6,50,10) \\
.70, .86\end{array}$ & $\begin{array}{l}\mathrm{BP}(2,1,0) \\
97,1,0\end{array}$ & $\begin{array}{l}\mathrm{RB}(7,50,0) \\
.60, .72\end{array}$ & $\begin{array}{l}\mathrm{RB}(6,50,3) \\
.56, .71 \\
\end{array}$ \\
\hline Level \#4 & $\begin{array}{l}\text { Net Type } \\
\text { C, CR } \\
\end{array}$ & & & & $\begin{array}{l}\mathrm{BP}(2,1,0) \\
.97,98 \\
\end{array}$ & $\begin{array}{l}\mathrm{BP}(7,4,0) \\
87, .90 \\
\end{array}$ & $\begin{array}{l}\mathrm{AB}(6,50,0) \\
95, .97\end{array}$ & $\begin{array}{l}\mathrm{BP}(2,1,0) \\
.97, .97 \\
\end{array}$ & $\begin{array}{l}B P(7,6,0) \\
93,93\end{array}$ & $\begin{array}{l}\mathrm{AB}(6,50,0) \\
.95, .97\end{array}$ & $\begin{array}{l}\mathrm{AB}(1,20,0) \\
.21, \ldots 4\end{array}$ & & & $\begin{array}{l}\mathrm{BP}(2,1,0) \\
97, \quad 1.0\end{array}$ & $\begin{array}{l}A B(7,50,0) \\
64, .79\end{array}$ & $\begin{array}{l}\mathrm{RB}(6,50,10) \\
.72, .84\end{array}$ & $\begin{array}{l}\mathrm{BP}(2,1,0) \\
.97, .99\end{array}$ & $\begin{array}{l}\mathrm{AB}(7,50,0) \\
.60, .75\end{array}$ & $\begin{array}{l}\mathrm{RB}(6,50,3) \\
.55, .70\end{array}$ \\
\hline Level \#5 & $\begin{array}{l}\text { Net Type } \\
\propto C, C B \\
\end{array}$ & & $\therefore$ & & $\begin{array}{l}\mathrm{BP}(2,1,0) \\
.97,99 \\
97,9\end{array}$ & $\begin{array}{l}\mathrm{BP}(7,4,0) \\
.86,90\end{array}$ & $\begin{array}{l}\mathrm{AB}(6,50,3) \\
.96, .96 \\
\end{array}$ & $\begin{array}{l}\mathrm{BP}(2,1,0) \\
95, .94 \\
\end{array}$ & $\begin{array}{l}\mathrm{BP}(7,6,0) \\
91,94\end{array}$ & $\begin{array}{l}\mathrm{AB}(6,50,4) \\
.96, .97 \\
\end{array}$ & & & & $\begin{array}{l}\mathrm{BP}(2,1,0) \\
.94, .96\end{array}$ & $\begin{array}{l}\mathrm{AB}(7,50,3) \\
.72, .85\end{array}$ & $\begin{array}{l}\mathrm{RB}(6,50,0) \\
72, .84\end{array}$ & $\begin{array}{l}\mathrm{BP}(2,1,0) \\
.95, .98\end{array}$ & $\begin{array}{l}R B(7,50,0) \\
61 . .75\end{array}$ & $\begin{array}{l}R B(6,50,3) \\
60, .77\end{array}$ \\
\hline Level \#6 & $\begin{array}{l}\text { Net Type } \\
\text { Cc,CR }\end{array}$ & & & & $\begin{array}{l}\mathrm{BP}(2,1,0) \\
.97,1,0\end{array}$ & $\begin{array}{l}\operatorname{BP}(7,4,0) \\
.91, .95\end{array}$ & \begin{tabular}{|l}
$\mathrm{RB}(6,50,0)$ \\
$.85, .96$
\end{tabular} & $\begin{array}{l}B P(2,1,0) \\
-93, .92 \\
\end{array}$ & $\begin{array}{l}B P(7,6,0) \\
.91,96 \\
\end{array}$ & $\begin{array}{l}\mathrm{AB}(6,50,0) \\
.94, .95\end{array}$ & & & & & & & $\begin{array}{l}\mathrm{BP}(2,1,0) \\
96,99\end{array}$ & $\begin{array}{l}\mathrm{AB}(2,50,0) \\
49, .65\end{array}$ & $\begin{array}{l}\mathrm{AB}(4,50,3) \\
.52, .72\end{array}$ \\
\hline Level \#7 & $\begin{array}{l}\text { Net Type } \\
\text { CC,CR } \\
\end{array}$ & & & & & & & $\begin{array}{l}\mathrm{BP}(2,1,0) \\
.90, .97 \\
\end{array}$ & $\begin{array}{l}\mathrm{BP}(7,6,0) \\
.92, .96 \\
\end{array}$ & $\begin{array}{l}\mathrm{RB}(6,50,0) \\
.93, .96\end{array}$ & & & & & & & & & \\
\hline EM Total & $\begin{array}{l}\text { Net Type } \\
\text { OC,CA } \\
\end{array}$ & & $\begin{array}{l}\text { AB( }(8,50,5) \\
.77, .87 \\
\end{array}$ & & & $\begin{array}{l}\mathrm{BP}(28,27,1 \\
.95, .96 \\
\end{array}$ & & & $\begin{array}{l}\mathrm{BP}(28,27,0 \\
.99, .99 \\
\end{array}$ & & & $\begin{array}{l}\mathrm{RB}(7,50,0) \\
.52, .55\end{array}$ & & & $\begin{array}{l}B P(12,11,5) \\
.67, .83\end{array}$ & & & $\begin{array}{l}\text { BP }(12,11,5) \\
.52, .67\end{array}$ & \\
\hline Mag Total & $\begin{array}{l}\text { Net Type } \\
\text { CC,CA }\end{array}$ & & $\begin{array}{l}\mathrm{RB}(8,50,5) \\
.89, .93 \\
\end{array}$ & & & $\begin{array}{l}\text { BP }(14,13,0 \\
.91, .94 \\
\end{array}$ & & & $\begin{array}{l}\mathrm{BP}(16,15,0 \\
.93, .97 \\
\end{array}$ & & & & & & $\begin{array}{l}\mathrm{AB}(12,50,0) \\
.62, .80\end{array}$ & & & $\begin{array}{l}\mathrm{RB}(10,50,0) \\
.46, .65\end{array}$ & \\
\hline Total & $\begin{array}{l}\text { Net Type } \\
\infty, C A \\
\end{array}$ & & $\begin{array}{l}\mathrm{BP}(16,15,8) \\
.86, .93 \\
\end{array}$ & & & $\begin{array}{l}\mathrm{BP}(42,41,0 \\
.95, .97\end{array}$ & & & $\begin{array}{l}\mathrm{BP}(44,43,1 \\
.99,1.0 \\
\end{array}$ & & & & & & $\begin{array}{l}\mathrm{BP}(18,17,8) \\
.69, .84\end{array}$ & & & $\begin{array}{l}\mathrm{BP}(36,35,1 \\
.78, .86\end{array}$ & \\
\hline & - Experime & nts 586 were & split in half & & & & & & & & & EM T2* & $\begin{array}{l}\text { Net Type } \\
\text { RMS, CA } \\
\end{array}$ & & $\begin{array}{l}\mathrm{BP}(12,11,6) \\
.80, .89\end{array}$ & & & $\begin{array}{l}\mathrm{BP}(14,13,7) \\
.78, .85 \\
\end{array}$ & \\
\hline & & & & & & & & & & & & Total 2 & $\begin{array}{l}\text { Net Type } \\
\text { PMS, CA }\end{array}$ & & $\begin{array}{l}\mathrm{BP}(18,17,8) \\
.77, .86\end{array}$ & & & & \\
\hline & & & & & & & & & & & & $E M 1+E M 2$ & $\begin{array}{l}\text { Net Type } \\
\text { RMS, CA } \\
\end{array}$ & & & & & $\begin{array}{l}\mathrm{BP}(26,25.1 \\
.77, .87 \\
\end{array}$ & \\
\hline
\end{tabular}


paradigm used, the size of the network (input, hidden layer one, hidden layer two), the correlation coefficient between desired and actual output, and the percentage of the output correctly classified are shown. Rows labeled EM total, magnetics total, and total, correspond to combining all the levels for each sensor and both sensors in a single pattern vector. Objects were removed between scan levels in Experiments 5 and 6 so when the scan levels were combined there was a problem with conflicting information in the pattern vector. One portion of the pattern vector indicated the presence of a target and another portion of the vector indicated the absence of the target. We avoided the problem by using one set of networks, EM1 and Total 1, for levels 0 through 2, and EMI T2 and Total 2 for levels 3 through 5 or 6.

In Table 5-11, Network type is the network design used and implies the paradigm and size of the network. For example BP( $a, b, c)$ means a back propagation network with "a" input, the size of the first hidden layer is " $b$," and the size of the second hidden layer is "c." All networks had one output unless otherwise noted.

The classification rate (CR) is the average percentage of samples correctly classified for both classes. If the background class had 100 training samples and all were correctly classified, and if the target class had five points and only one was missed, the CR would be the average of $100 \%$ and $80 \%$ or $90 \%$ even though only one point out of 105 was missed. When the number of samples in each class is equal, the CR can underestimate the total percent classified. We typically had training sets with $75 \%$ of the points in the background class and $25 \%$ in the target class. Further experiments should be conducted to determine if fewer background training samples can be used.

Blank sections in this table represent missing information or unusable data. For example, there was an insufficient number of levels gathered on Experiment 1 to test combinations of the magnetic and EM sensor data. Likewise, the data from Experiment 4 was not useful.

As the grids presented above and the CR numbers in Table 5-11 show, the networks were able to locate almost all targets on which they were trained. The notable exception is Experiment 6, which contained some long horizontal objects displaying strange bi-polar signatures in the survey data. This is evidenced by the CR values $(0.5-0.6)$ in Table 5-11 for the EM and total entries on Experiment 6.

Tables 5-12 through 5-15 present information from the interexperiment testing done throughout this research (testing networks on different experiments than those used for training). Table 5-12 shows the results from testing a network trained only on Experiment 2 with data from all other experiments. The columns represent the level and sensor used ("total" means combined magnetic and EM data for training) and the rows represent the test data. For example, the Mag column under the heading Level-\#0 indicates a network trained on the magnetic data from Experiment 2, level 0 . The rows in this table represent the test data files used. For example, the level $\# 5$ row within the Ex $\# 2$ rows means that the test files used came from Experiment 2, level 5. When the rows intersect a Mag column, the magnetic data from that row location were used for testing; when the rows intersect an EM column, then EM data was used for testing and so forth. Tables 5-13 through 5-15 represent Experiments 3, 5, and 6, respectively and are set up the same way. The values shown in these tables represent the correlation coefficient and CR, respectively for the test results. Caution should be used in comparing the magnetic results to the EM and EM plus magnetics because the magnetic data were preprocessed, which may have biased the results as discussed above. When EM and magnetics were combined, the magnetics data were not preprocessed. The major finding from this task was that no single experiment contained enough variability to allow it 
Table 5-12. Interexperiment testing with neural network trained with Experiment 2 data.

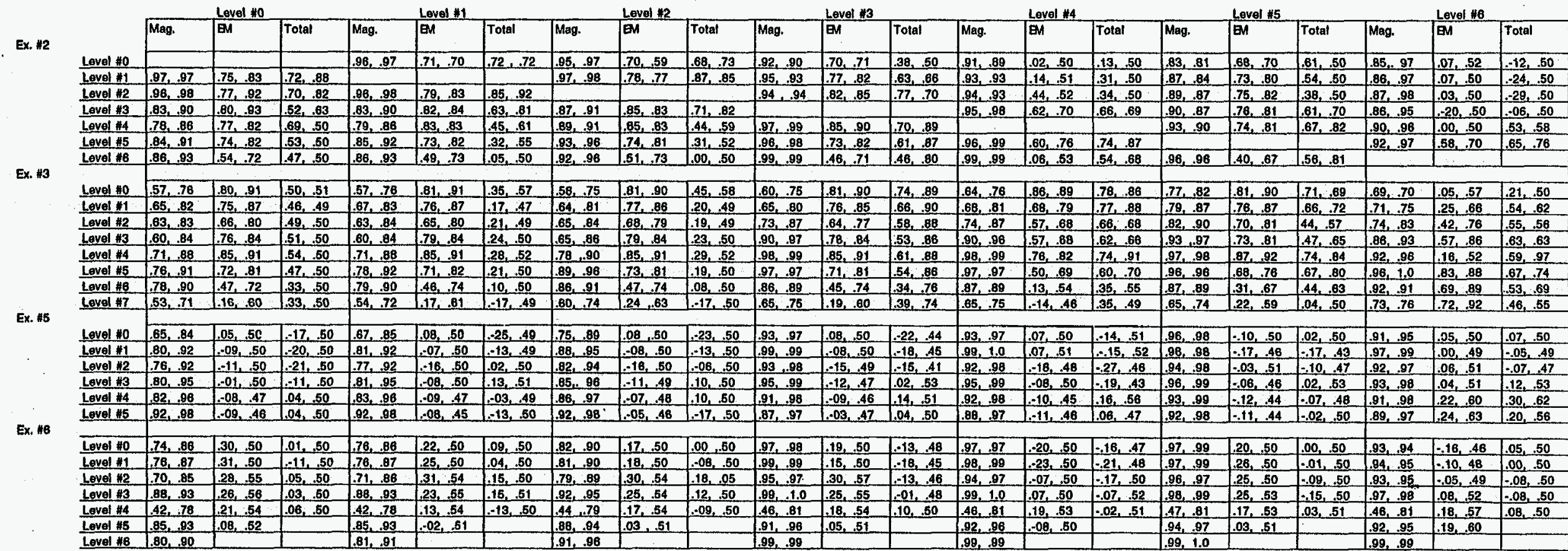


Table 5-13. Interexperiment testing with neural network trained with Experiment 3 data.

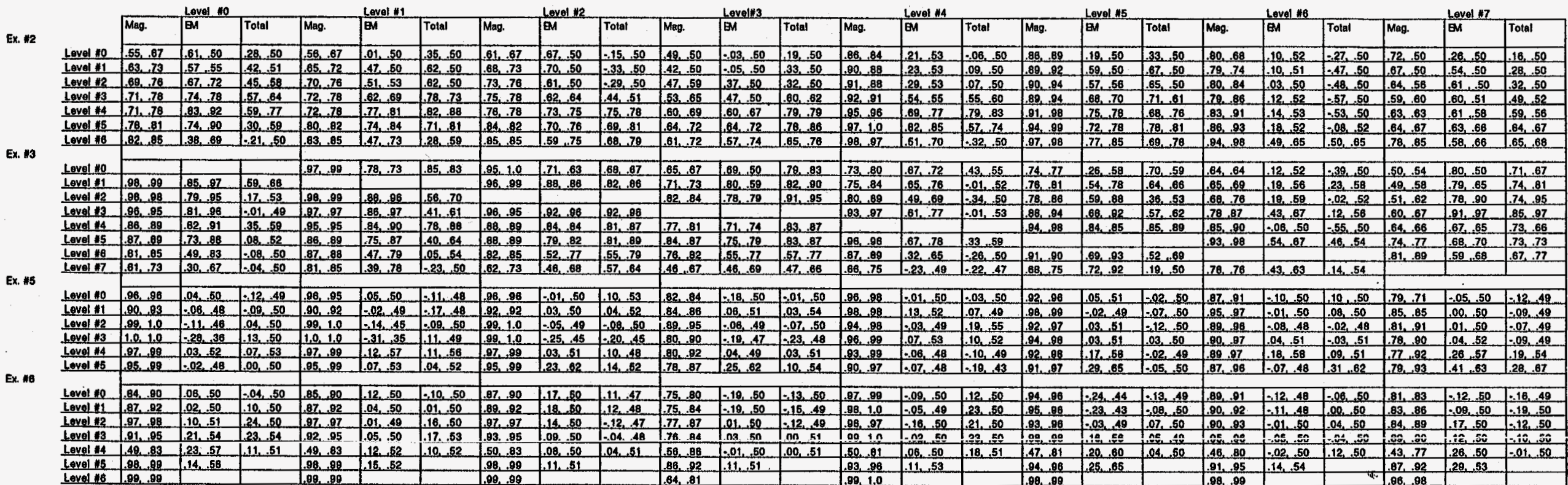


Table 5-14. Interexperiment testing with neural network trained with Experiment 5 data.

Ex. \#2

\begin{tabular}{|c|c|c|c|c|c|c|c|c|c|c|c|c|}
\hline & Level \#0 & & Level \#1 & & Level \#2 & & Level\#3 & & Level \#4 & & Level \#5 & \\
\hline & $\mathrm{EM}$ & Total & $E M$ & Total & $\mathrm{BM}$ & Total & en & Total & EM & Total & $\mathrm{BM}$ & Total \\
\hline Level \#0 & $-.25, .50$ & $-.29, .50$ & $-.54, .50$ & $-.58, .50$ & $-.02, .50$ & $.38, .50$ & $.29, .50$ & $-.11, .50$ & $.06, .50$ & $-.14, .50$ & $-.19, .50$ & $-.07, .50$ \\
\hline Level \#1 & $.41, .50$ & $-.10, .50$ & $.44, .50$ & $-.58, .50$ & $.06, .50$ & $.37, .50$ & $.54, .50$ & $-.10, .50$ & $.00, .50$ & $-.41, .50$ & $. .46, .50$ & $-.46, .50$ \\
\hline Level \#2 & $.40, .50$ & $-.11, .50$ & $-.07, .50$ & $-.59, .50$ & $.26, .50$ & $.37, .50$ & $.26, .50$ & $-.14, .50$ & $.24, .50$ & $-.43, .50$ & $-.61, .50$ & $-.43, .50$ \\
\hline Level \#3 & $.32, .49$ & $.10, .50$ & $-.05, .50$ & $.10, .50$ & $.41, .64$ & $.30, .53$ & $.06, .50$ & $-.11, .50$ & $.33, .50$ & $-.26, .50$ & $-.57, .50$ & $-.26, .50$ \\
\hline Level \#4 & $20 . .41$ & $.30, .50$ & $-.05, .49$ & $-.11, .48$ & $.35, .70$ & $-.02, .45$ & $.01, .50$ & $-.28, .50$ & $.61, .51$ & $-.38, .50$ & $.04, .51$ & $.18, .51$ \\
\hline Level \#5 & $.23, .44$ & $.38, .49$ & $-.14, .44$ & $.15, .51$ & $.34, .74$ & $.03, .48$ & $.24, .50$ & $-.19, .50$ & $.61, .52$ & $-.25, .50$ & $.43, .55$ & $.47, .52$ \\
\hline Level \#6 & $.30, .45$ & $.49, .50$ & $-.15, .46$ & $.24, .49$ & $.41, .82$ & $.06, .47$ & $.24, .55$ & $.29, .60$ & $.57, .52$ & $.24, .58$ & $.49, .65$ & $.52, .60$ \\
\hline Level \#0 & $48, .49$ & $.05, .45$ & $-.06, .50$ & $.00, .47$ & $.38, .72$ & $-.08, .49$ & $.05, .50$ & $-.07, .50$ & $.64, .54$ & $-.20, .50$ & $.32, .50$ & $.47, .50$ \\
\hline Level \#1 & $.53, .48$ & $.18, .47$ & $-.04, .49$ & $-.05, .47$ & $.51, .83$ & $.03, .49$ & $-.13, .50$ & $-.15, .50$ & $.50, .52$ & $-.34, .50$ & $.59, .64$ & $.60, .55$ \\
\hline Level \#2 & $.55, .50$ & $.40, .50$ & $.02, .49$ & $.21, .48$ & $.57, .88$ & $.12, .49$ & $-.07, .50$ & $-.13, .50$ & $.43, .50$ & $-.34, .50$ & $.82, .79$ & $.62, .57$ \\
\hline Level \#3 & $.35, .46$ & $.43, .50$ & $-.10, .48$ & $.13, .48$ & $.45, .85$ & $.14, .48$ & $-.19, .50$ & $-.10, .50$ & $.22, .50$ & $-.24, .50$ & $.82, .75$ & $.70, .61$ \\
\hline Level \#4 & $.34, .47$ & $.21, .47$ & $-.01, .49$ & $\because .01, .46$ & $.35, .75$ & $-.02, .47$ & $.09, .50$ & $-.09, .50$ & $.65, .61$ & $-.24, .50$ & $.36, .52$ & $.49, .50$ \\
\hline Level \#5 & $.29, .46$ & $.42, .49$ & $-.10, .47$ & $.16, .48$ & $.31, .76$ & $.11, .51$ & $.03, .50$ & $-.01, .50$ & $.52, .55$ & $-.15, .51$ & $.62, .66$ & $.59, .60$ \\
\hline Level \#6 & $.24, .47$ & $.29, .50$ & $-.09, .47$ & $.23, .49$ & $.28, .77$ & $.13, .49$ & $.21, .57$ & $.05, .50$ & $.17, .50$ & $.04, .52$ & $.59, .73$ & $. .54, .65$ \\
\hline Level \#7 & $.22, .45$ & $.37, .50$ & $-.07, .47$ & $.46, .50$ & $.45, .84$ & $.33, .49$ & $-.19, .49$ & $-.20, .50$ & $.53, .68$ & $-.29, .50$ & $.78, .89$ & $.63, .63$ \\
\hline Level \#0 & & & $.21, .56$ & $.27, .58$ & $-.23, .40$ & $-.12, .48$ & $-.13, .50$ & $.04, .50$ & $-.11, .50$ & $-.19, .50$ & $-.10, .50$ & $-.14, .50$ \\
\hline Level \#1 & $.30, .63$ & $.24, .53$ & & & $-.06, .49$ & $.11, .52$ & $-.15, .49$ & $.14, .50$ & $-.10, .50$ & $-.05, .50$ & $.03, .50$ & $-.13, .50$ \\
\hline Level \#2 & $-.01, .50$ & $-.10, .49$ & $.14, .53$ & $10, .49$ & & & $-.09, .50$ & $.39, .50$ & $-.17 ; .50$ & $.22, .50$ & $.07, .50$ & $.01, .50$ \\
\hline Level \#3 & $-.10, .50$ & $.09, .50$ & $.04, .50$ & $-.10, .50$ & $.02, .53$ & $-.11, .49$ & & & $-.03, .48$ & $.73, .85$ & $.12, .52$ & $.16, .52$ \\
\hline Level \#4 & $.21, .50$ & $.12, .50$ & $-.07, .50$ & $.17, .50$ & $.16, .58$ & $.09, .49$ & $-.21, .47$ & $-.07, .50$ & & & $.29, .57$ & $.30, .58$ \\
\hline Level \#5 & $.12, .50$ & $.11, .50$ & $-.02, .50$ & $.22, .50$ & $.34, .69$ & $1.13, .49$ & $-.10, .50$ & $.04, .51$ & $.07, .53$ & $.04, .51$ & & \\
\hline Level \#0 & $-.15, .50$ & $-.15, .49$ & $-.11, .50$ & $-.16, .50$ & $-.18, .45$ & $-.09, .45$ & $-.11, .50$ & $-.01, .50$ & $.18, .50$ & $.09, .50$ & $-.10, .50$ & $-.14, .50$ \\
\hline Level \#1 & $-.15, .50$ & $-.30, .50$ & $-.24, .50$ & $-.28, .50$ & $-.20, .43$ & $-.10, .43$ & $-.03, .50$ & $-.04, .50$ & $.11, .50$ & $.14, .50$ & $-.05, .50$ & $-.05, .50$ \\
\hline Level \#2 & $-.08, .50$ & $-.25, .50$ & $-.24, .50$ & $-.25, .50$ & $-.18, .41$ & $.00, .50$ & $.06, .50$ & $-.10, .50$ & $-.06, .50$ & $-.01, .50$ & $.14, .50$ & $.01, .50$ \\
\hline Level \#3 & $.07, .50$ & $-.25, .47$ & $-.18, .50$ & $-.19, .49$ & $-.13, .44$ & $.06, .53$ & $-.03, .50$ & $-.06, .50$ & $-.13, .50$ & $-.04, .50$ & $-.08, .50$ & $-.06, .50$ \\
\hline Level \#4 & $.11, .50$ & $-.15, .49$ & $.06, .50$ & $-.08, .50$ & $.05, .49$ & $.06, .49$ & $.21, .50$ & $.11,: 50$ & $-.10, .50$ & $.15, .50$ & $-.02, .50$ & $.05, .50$ \\
\hline Level \#5 & $.08, .50$ & & $.05, .50$ & & $.03, .51$ & & $.06, .51$ & & $.22, .53$ & & $.28, .55$ & \\
\hline Level \#6 & & & & & & & & & & & & \\
\hline
\end{tabular}


Table 5-15. Interexperiment testing of neural network trained with Experiment 6 data.

Ex. \#2

\begin{tabular}{|c|c|c|c|c|c|c|c|c|c|c|c|}
\hline \multirow[b]{3}{*}{ Level \#0 } & \multicolumn{2}{|l|}{ Level \#0 } & \multicolumn{2}{|l|}{ Level \#1. } & \multicolumn{2}{|l|}{ Level \#2 } & \multicolumn{2}{|l|}{ Level\#3 } & \multicolumn{2}{|l|}{ Level \#4 } & \multirow{2}{*}{$\begin{array}{l}\text { Level \#5 } \\
\text { EM }\end{array}$} \\
\hline & $\mathrm{EM}$ & Total & $B M$ & Total & EM & Total & $E M$ & Total & $\mathrm{BM}$ & Total & \\
\hline & $-.26, .50$ & $-.02, .50$ & $-.27, .50$ & $-.01, .50$ & $.54, .51$ & $.52, .60$ & $.69, .69$ & $.70, .81$ & $.65, .77$ & $.61, .58$ & $.14, .52$ \\
\hline Level \#1 & $.10, .50$ & $.41, .50$ & $-.23, .50$ & $.08, .50$ & $.44, .50$ & $.37, .53$ & $.75, .82$ & $.74, .82$ & $.75, .90$ & $.67, .56$ & $.16, .52$ \\
\hline Level \#2 & $.05, .50$ & $.38, .50$ & $-.30, .47$ & $.03, .50$ & $.23, .52$ & $.37, .52$ & $.75, .85$ & $.72, .81$ & $.73, .90$ & $.65, .57$ & $.17, .51$ \\
\hline Level \#3 & $.43, .50$ & $.72, .50$ & $-.27, .49$ & $.08, .48$ & $.32, .52$ & $.29, .54$ & $.81, .94$ & $.78, .92$ & $.74, .92$ & $.68,63$ & $.30, .51$ \\
\hline Level \#4 & $.60, .50$ & $.67, .50$ & $.16, .50$ & $.53, .50$ & $.12, .50$ & $.17, .50$ & $.59, .77$ & $.63, .76$ & $.66, .86$ & $.66, .62$ & $.60, .71$ \\
\hline Level \#5 & $.54, .50$ & $.61, .50$ & $.06, .50$ & $.33, .49$ & $-.01, .50$ & $.13, .50$ & $.62, .75$ & $.63, .79$ & $.43, .77$ & $.38, .49$ & $.46, .78$ \\
\hline Level \#6 & $.43, .50$ & $.47, .50$ & $.21, .50$ & $.38, .50$ & $-.13, .50$ & $.01, .50$ & $.50, .84$ & $.58, .84$ & $.05, .58$ & $.20, .53$ & $.49, .80$ \\
\hline Level \#0 & $.30, .49$ & $.57, .50$ & $-.13, .50$ & $.15, .49$ & $.08, .50$ & $-.06, .50$ & $.74, .89$ & $.75, .91$ & $.61, .88$ & $.60, .77$ & $.59, .77$ \\
\hline Level \#1 & $.56, .50$ & $.70, .50$ & $-.07, .50$ & $.28, .50$ & $.00, .50$ & $-.09, .49$ & $.68, .88$ & $.67, .89$ & $.48, .81$ & $.51, .69$ & $.67, .89$ \\
\hline Level \#2 & $.54, .50$ & $.62, .50$ & $-.11, .50$ & $.23, .50$ & $-.10, .50$ & $-.12, .48$ & $.64, .92$ & $.64, .90$ & $.47, .82$ & $.49, .54$ & $.64, .91$ \\
\hline Level \#3 & $.55, .50$ & $.60, .50$ & $.23, .50^{\circ}$ & $.56, .50$ & $.16, .50$ & $.41, .50$ & $.68, .94$ & $.69, .92$ & $.51, .86$ & $.47, .52$ & $.65, .91$ \\
\hline Level \#4 & $.62, .50$ & $.68, .50$ & $.03, .50$ & $.45, .50$ & $.02, .50$ & $-.03, .50$ & $.67, .85$ & $.73, .88$ & $.59, .88$ & $.62, .57$ & $.60, .86$ \\
\hline Level \#5 & $.51, .50$ & $.58, .50$ & $-.02, .50$ & $.34, .50$ & $-.18, .50$ & $-.03, .50$ & $.58, .83$ & $.65, .87$ & $.28, .71$ & $.42, .53$ & $.54, .82$ \\
\hline Level \#6 & $.31, .50$ & $.35, .50$ & $.09, .50$ & $.33, .50$ & $-.08, .50$ & $.07, .50$ & $.45, .84$ & $.45, .86$ & $.30, .77$ & $.34, .60$ & $.36, .77$ \\
\hline Level \#7 & $.37, .50$ & $.40, .50$ & $-.05, .50$ & $.37, .50$ & $-.15, .50$ & $-.02, .50$ & $.38, .80$ & $.47, .81$ & $.01, .58$ & $.18, .50$ & $.46, .78$ \\
\hline Level \#0 & $-.22, .50$ & $-.21 \quad .50$ & $-.31, .50$ & $-.25, .50$ & $-.24, .50$ & $-.30, .50$ & $-.12, .47$ & $-.13, .47$ & $-.32, .34$ & $-.37, .43$ & $-.29, .42$ \\
\hline Level \#1 & $-.18, .50$ & $-.16, .50$ & $-.29, .50$ & $-.18, .50$ & $.02, .50$ & $-.18, .50$ & $-.21, .47$ & $-.18, .46$ & $-.21, .41$ & $-.12, .52$ & $-.05, .48$ \\
\hline Level \#2 & $-.10 ; .50$ & $-11 ;, 50$ & $-19,50$ & $-.08, .50$ & $.25, .50$ & $.01, .50$ & $.24, \quad .42$ & $-.18, .44$ & $-.08, .52$ & $-.07, .51$ & $-.14, .44$ \\
\hline Level \#3 & $.07, .50$ & $.05, .50$ & $.06, .50$ & $.08, .50$ & $.01, .50$ & $.12, .50$ & $-.13, .43$ & $-.14, .42$ & $.00, .48$ & $.03, .49$ & $-.12, .46$ \\
\hline Level \#4 & $.19, .50$ & $.20, .50$ & $.13, .50$ & $.17, .50$ & $-.05, .50$ & $.07, .50$ & $.24, .60$ & $.23, .57$ & $-.07, .53$ & $.06, .52$ & $.33, .63$ \\
\hline Level \#5 & $.11, .50$ & $.14, .50$ & $.06, .50$ & $.11, .50$ & $-.01, .50$ & $.06, .50$ & $.18, .57$ & $.16, .53$ & $-.05, .52$ & $.03, .50$ & $.34, .60$ \\
\hline Level \#0 & & & $.31, .55$ & $.28, .54$ & $-.01, .50$ & $.18, .50$ & $-.22, .45$ & $-.14, .49$ & $.02, .50$ & $-.01, .47$ & $-.14, .50$ \\
\hline Level \#1 & $.12, .52$ & $.17, .52$ & & & $.10, .49$ & $.31, .50$ & $-.13, .47$ & $-.11, .48$ & $.14, .50$ & $.12, .51$ & $-.11, .50$ \\
\hline Level \#2 & $-.04, .51$ & $-.09, .50$ & $.13, .50$ & $.08, .50$ & & & $.21, .57$ & $.17, .55$ & $.27, .55$ & $.20, .55$ & $-.10, .50$ \\
\hline Level \#3 & $-.16, .50$ & $-.05, .50$ & $-.02, .50$ & $.10, .50$ & $.07, .53$ & $.01, .53$ & & & $.25, .55$ & $.21, .55$ & $-.18, .50$ \\
\hline Level \#4 & $-.05, .50$ & $.10, .50$ & $.09, .50$ & $.20, .50$ & $.14, .52$ & $.15, .52$ & $.30, .59$ & $.31, .67$ & & & $.05, .50$ \\
\hline Level \#5 & $-.01, .50$ & & $.19, .50$ & & $.12, .50$ & & $.27, .65$ & & $.23, .66$ & & \\
\hline
\end{tabular}


to accurately locate objects from other experiments. Neural networks are excellent interpolators but poor extrapolators. It is imperative in constructing a training set that the data bound the expected ranges to be encountered during testing. Final classification networks were trained using data including objects 6 , $7,15,16,17,20,21,23,24,25,26$, and 28 as discussed above (and in Appendix A). The robustness of the results is unknown since there is little additional data for testing use.

In general, the wooden boxes were located by the networks in Experiment 5 but individual boxes were generally not distinguishable in the gridded output. The horizontal pail was occasionally identified by the networks for some scan levels and some sensors, but the vertical pail was never located. When the experiments were combined, neither pail was identified. Part of the reason for this discrepancy is due to the number of training points included for each target. The orientation of the horizontal pail relative to the scan direction allowed more data points to be collected over it than over the vertical pail. Eleven points were used for training on the horizontal pail and only four for the vertical pail. When the experiments were combined, the low amplitude response from the pails was more indicative of the overall background response than the other targets.

The horizontal bars, pipes, and C-channel in Experiment 6 were well resolved when training took place only on Experiment 6 data. When data from multiple experiments were combined, objects 31, 32, 33, and 34 were not as well resolved. It is clear that the networks were confused by the complicated signatures in Experiment 6 when they were allowed to see only one data point at a time. We could force a network to find a set of weights to distinguish each object if we only used data from Experiment 6 but as soon as we used additional data conflicting patterns arose and we could not distinguish objects 31,32 , 33 , and 34 as easily.

The network gridded output suggests that the EM survey more accurately finds targets than the magnetic survey. However, if any EM component is missing, the network performance is noticeably hurt. When EM and magnetic survey data are combined, the network performance is not damaged when one or two EM components are unavailable. Tables 5-16 and 5-17 are selected results from a study of which components of which surveys are most significant. Individual box labels represent the experiment and level for which the tests were done. For example, E2L3EM indicates Experiment 2, level 3, EM data. EM refers to only the four EM components, while TOT means that the two magnetic components were added to the test set. S2, S3, S4, and S5 reflect the EM sensor designations in the digface data files and also the input to the networks. $M$ represents both magnetic components. The checked boxes indicate the network (PEs) that were disabled for the particular case. The results column contains the correlation coefficient between desired and actual output and the classification rate, respectively.

From Tables 5-16 and 5-17, it was observed that the test sets including magnetic components were less affected by disabling certain EM survey components. Compared to the baseline values when components were disabled, adding magnetic data almost always improved the results when one or more EM components were disabled. When the baseline results were good (e.g., Experiment 2), the loss of a single component could severely degrade the performance and the loss of two or more components continued to worsen the results. When the baseline performance was poorer (e.g., Experiment 5), the loss of components had little or no effect. Because of object dependence, the importance of one component over another could not be determined. 
Table 5-16. Summary of testing for importance of survey components in neural network training and testing.

E3LOEM
\begin{tabular}{|l|l|l|l|l|}
\hline$S 2$ & $S 3$ & $S 4$ & $S 5$ & Results \\
\hline & & & & $.95, .94$ \\
\hline$X$ & & & & $.93, .96$ \\
\hline$X$ & $X$ & & & $.92, .94$ \\
\hline$X$ & $X$ & $X$ & & $.84, .89$ \\
\hline$X$ & & $X$ & $X$ & $.35, .54$ \\
\hline$X$ & $X$ & & $X$ & $.73, .81$ \\
\hline$X$ & & & $X$ & $.75, .81$ \\
\hline$X$ & & $X$ & & $.84, .87$ \\
\hline & $X$ & $X$ & & $.89, .93$ \\
\hline & $X$ & $X$ & $X$ & $.48, .50$ \\
\hline & $X$ & & $X$ & $.76, .83$ \\
\hline & $X$ & & & $.93, .96$ \\
\hline & & $X$ & & $.88, .92$ \\
\hline & & $X$ & $X$ & $.74, .85$ \\
\hline & & & $X$ & $.82, .85$ \\
\hline
\end{tabular}

\section{E3LOTOT}

[Dissabled

\begin{tabular}{|l|l|l|l|l|l|}
\hline$S 2$ & $S 3$ & $S 4$ & $S 5$ & $M$ & Results \\
\hline & & & & & $.94, .98$ \\
\hline$X$ & & & & & $.95, .97$ \\
\hline$X$ & $X$ & & & & $.93, .95$ \\
\hline$X$ & $X$ & $X$ & & & $.89, .89$ \\
\hline$X$ & & $X$ & $X$ & & $.83, .89$ \\
\hline$X$ & $X$ & & $X$ & & $.84, .88$ \\
\hline$X$ & & & $X$ & & $.90, .93$ \\
\hline$X$ & & $X$ & & & $.94, .96$ \\
\hline & $X$ & $X$ & & & $.95, .96$ \\
\hline & $X$ & $X$ & $X$ & & $.85, .90$ \\
\hline & $X$ & & $X$ & & $.90, .93$ \\
\hline & $X$ & & & & $.95, .98$ \\
\hline & & $X$ & & & $.95, .98$ \\
\hline & & $X$ & $X$ & & $.84, .92$ \\
\hline & & & $X$ & & $.90, .92$ \\
\hline & & & & $X$ & $.96, .98$ \\
\hline
\end{tabular}

E5L1EM

Dissabled

\begin{tabular}{|l|l|l|l|l|}
\hline$S 2$ & $S 3$ & $S 4$ & $S 5$ & Results \\
\hline & & & & $.66, .82$ \\
\hline$X$ & & & & $.66, .82$ \\
\hline$X$ & $X$ & & & $.58, .78$ \\
\hline$X$ & $X$ & $X$ & & $.45, .69$ \\
\hline$X$ & & $X$ & $X$ & $.25, .57$ \\
\hline$X$ & $X$ & & $X$ & $.36, .58$ \\
\hline$X$ & & & $X$ & $.52, .65$ \\
\hline$X$ & & $X$ & & $.58, .76$ \\
\hline & $X$ & $X$ & & $.55, .77$ \\
\hline & $X$ & $X$ & $X$ & $.22, .56$ \\
\hline & $X$ & & $X$ & $.47, .69$ \\
\hline & $X$ & & & $.61, .80$ \\
\hline & & $X$ & & $.62, .77$ \\
\hline & & $X$ & $X$ & $.35, .59$ \\
\hline & & & $X$ & $.56, .69$ \\
\hline
\end{tabular}

E5L5EM

Dissabled
\begin{tabular}{|l|l|l|l|l|}
\hline$S 2$ & $S 3$ & $S 4$ & $S 5$ & Results \\
\hline & & & & $.72, .85$ \\
\hline$X$ & & & & $.67, .83$ \\
\hline$X$ & $X$ & & & $.60, .73$ \\
\hline$X$ & $X$ & $X$ & & $.44, .67$ \\
\hline$X$ & & $X$ & $X$ & $.32, .55$ \\
\hline$X$ & $X$ & & $X$ & $.39, .65$ \\
\hline$X$ & & & $X$ & $.60, .74$ \\
\hline$X$ & & $X$ & & $.60, .75$ \\
\hline & $X$ & $X$ & & $.63, .81$ \\
\hline & $X$ & $X$ & $X$ & $.39, .65$ \\
\hline & $X$ & & $X$ & $.64, .76$ \\
\hline & $X$ & & & $.72, .83$ \\
\hline & & $X$ & & $.70, .85$ \\
\hline & & $X$ & $X$ & $.54, .75$ \\
\hline & & & $X$ & $.68, .83$ \\
\hline
\end{tabular}

Dissabled

\begin{tabular}{|l|l|l|l|l|l|}
\hline$S$ & $S 3$ & $S 4$ & $S 5$ & $M$ & Results \\
\hline & & & & & $.67, .82$ \\
\hline$X$ & & & & & $.68, .82$ \\
\hline$X$ & $X$ & & & & $.65, .81$ \\
\hline$X$ & $X$ & $X$ & & & $.64, .70$ \\
\hline$X$ & & $X$ & $X$ & & $.60, .74$ \\
\hline$X$ & $X$ & & $X$ & & $.58, .75$ \\
\hline$X$ & & & $X$ & & $.66, .82$ \\
\hline$X$ & & $X$ & & & $.66, .80$ \\
\hline & $X$ & $X$ & & & $.64, .77$ \\
\hline & $X$ & $X$ & $X$ & & $.59, .80$ \\
\hline & $X$ & & $X$ & & $.62, .80$ \\
\hline & $X$ & & & & $.64, .75$ \\
\hline & & $X$ & & & $.65, .82$ \\
\hline & & $X$ & $X$ & & $.65, .80$ \\
\hline & & & $X$ & & $.66, .83$ \\
\hline & & & & $X$ & $.65, .83$ \\
\hline
\end{tabular}

\section{E5L5TOT}

\begin{tabular}{|c|c|c|c|c|c|}
\hline \multicolumn{6}{|c|}{ Disssabled } \\
\hline \multirow[t]{2}{*}{ SE } & S3 & S4 & S5 & $M$ & Results \\
\hline & & & & & $.72, .84$ \\
\hline$x$ & & & & & $.72, .81$ \\
\hline $\bar{x}$ & $x$ & & & & $.70, .80$ \\
\hline $\mathrm{x}$ & $x$ & $x$ & & & $.63, .72$ \\
\hline$x$ & & $x$ & $x$ & & $.58, .71$ \\
\hline$\underline{x}$ & $x$ & & $\mathrm{X}$ & & $.65, .77$ \\
\hline$x$ & & & $x$ & & $.69, .83$ \\
\hline$x$ & & $x$ & & & $.67, .82$ \\
\hline & $X$ & $x$ & & & $.68, .77$ \\
\hline & $x$ & $\bar{x}$ & X & & $.60, .76$ \\
\hline & $x$ & & $x$ & & $.67, .82$ \\
\hline & $x$ & & & & $.72, .85$ \\
\hline & & $x$ & & & $.68, .83$ \\
\hline & & $x$ & $x$ & & $.59, .75$ \\
\hline & & & $x$ & & $.71, .85$ \\
\hline & & & & $x$ & $.73, .84$ \\
\hline
\end{tabular}


Table 5-17. Summary of testing for importance of survey components in neural network training and testing.

E2L3EM

Dissabled

\begin{tabular}{|l|l|l|l|l|}
\hline $\mathrm{S} 2$ & $\mathrm{S3}$ & $\mathrm{S4}$ & $\mathrm{S5}$ & Results \\
\hline & & & & $.91, .95$ \\
\hline $\mathrm{X}$ & & & & $.90, .95$ \\
\hline $\mathrm{X}$ & $\mathrm{X}$ & & & $.85, .90$ \\
\hline $\mathrm{X}$ & $\mathrm{X}$ & $\mathrm{X}$ & & $.80, .83$ \\
\hline $\mathrm{X}$ & & $\mathrm{X}$ & $\mathrm{X}$ & $.48, .73$ \\
\hline $\mathrm{X}$ & $\mathrm{X}$ & & $\mathrm{X}$ & $.62, .77$ \\
\hline $\mathrm{X}$ & & & $\mathrm{X}$ & $.76, .80$ \\
\hline $\mathrm{X}$ & & $\mathrm{X}$ & & $.90, .95$ \\
\hline & $\mathrm{X}$ & $\mathrm{X}$ & & $.85, .89$ \\
\hline & $\mathrm{X}$ & $\mathrm{X}$ & $\mathrm{X}$ & $.43, .66$ \\
\hline & $\mathrm{X}$ & & $\mathrm{X}$ & $.68, .77$ \\
\hline & $\mathrm{X}$ & & & $.86, .92$ \\
\hline & & $\mathrm{X}$ & & $.91, .95$ \\
\hline & & $\mathrm{X}$ & $\mathrm{X}$ & $.57, .68$ \\
\hline & & & $\mathrm{X}$ & $.82, .91$ \\
\hline
\end{tabular}

E3L7EM

Dissabled

\begin{tabular}{|l|l|l|l|l|}
\hline $\mathrm{S} 2$ & $\mathrm{S3}$ & $\mathrm{S4}$ & $\mathrm{S} 5$ & Results \\
\hline & & & & $.92, .96$ \\
\hline $\mathrm{X}$ & & & & $.92, .95$ \\
\hline $\mathrm{X}$ & $\mathrm{X}$ & & & $.89, .89$ \\
\hline $\mathrm{X}$ & $\mathrm{X}$ & $\mathrm{X}$ & & $.00, .50$ \\
\hline $\mathrm{X}$ & & $\mathrm{X}$ & $\mathrm{X}$ & $.71, .88$ \\
\hline $\mathrm{X}$ & $\mathrm{X}$ & & $\mathrm{X}$ & $.79, .84$ \\
\hline $\mathrm{X}$ & & & $\mathrm{X}$ & $.87, .89$ \\
\hline $\mathrm{X}$ & & $\mathrm{X}$ & & $.70, .85$ \\
\hline & $\mathrm{X}$ & $\mathrm{X}$ & & $.85, .91$ \\
\hline & $\mathrm{X}$ & $\mathrm{X}$ & $\mathrm{X}$ & $.84, .92$ \\
\hline & $\mathrm{X}$ & & $\mathrm{X}$ & $.85, .91$ \\
\hline & $\mathrm{X}$ & & & $.86, .90$ \\
\hline & & $\mathrm{X}$ & & $.87, .96$ \\
\hline & & $\mathrm{X}$ & $\mathrm{X}$ & $.86, .93$ \\
\hline & & & $\mathrm{X}$ & $.88, .95$ \\
\hline
\end{tabular}

\section{E2L3TOT}

Dissabled

\begin{tabular}{|l|l|l|l|l|l|}
\hline $\mathrm{S} 2$ & S3 & S4 & S5 & $M$ & Results \\
\hline & & & & & $.93, .95$ \\
\hline $\mathrm{X}$ & & & & & $.94, .97$ \\
\hline $\mathrm{X}$ & $\mathrm{X}$ & & & & $.92, .94$ \\
\hline $\mathrm{X}$ & $\mathrm{X}$ & $\mathrm{X}$ & & & $.91, .96$ \\
\hline $\mathrm{X}$ & & $\mathrm{X}$ & $\mathrm{X}$ & & $.85, .89$ \\
\hline $\mathrm{X}$ & $\mathrm{X}$ & & $\mathrm{X}$ & & $.87, .91$ \\
\hline $\mathrm{X}$ & & & $\mathrm{X}$ & & $.90, .94$ \\
\hline $\mathrm{X}$ & & $\mathrm{X}$ & & & $.94, .96$ \\
\hline & $\mathrm{X}$ & $\mathrm{X}$ & & & $.93, .96$ \\
\hline & $\mathrm{X}$ & $\mathrm{X}$ & $\mathrm{X}$ & & $.85, .93$ \\
\hline & $\mathrm{X}$ & & $\mathrm{X}$ & & $.89, .93$ \\
\hline & $\mathrm{X}$ & & & & $.91, .93$ \\
\hline & & $\mathrm{X}$ & & & $.92, .95$ \\
\hline & & $\mathrm{X}$ & $\mathrm{X}$ & & $.86, .87$ \\
\hline & & & $\mathrm{X}$ & & $.87, .93$ \\
\hline & & & & $\mathrm{X}$ & $.93, .96$ \\
\hline
\end{tabular}

\section{E3L7TOT}

Dissabled

\begin{tabular}{|l|l|l|l|l|l|}
\hline S2 & $S 3$ & $S 4$ & $S 5$ & $M$ & Results \\
\hline & & & & & $.93, .96$ \\
\hline $\mathrm{X}$ & & & & & $.92, .92$ \\
\hline $\mathrm{X}$ & $\mathrm{X}$ & & & & $.93, .96$ \\
\hline $\mathrm{X}$ & $\mathrm{X}$ & $\mathrm{X}$ & & & $.85, .90$ \\
\hline $\mathrm{X}$ & & $\mathrm{X}$ & $\mathrm{X}$ & & $.84, .92$ \\
\hline $\mathrm{X}$ & $\mathrm{X}$ & & $\mathrm{X}$ & & $.89, .92$ \\
\hline $\mathrm{X}$ & & & $\mathrm{X}$ & & $.90, .94$ \\
\hline $\mathrm{X}$ & & $\mathrm{X}$ & & & $.89, .92$ \\
\hline & $\mathrm{X}$ & $\mathrm{X}$ & & & $.91, .93$ \\
\hline & $\mathrm{X}$ & $\mathrm{X}$ & $\mathrm{X}$ & & $.90, .92$ \\
\hline & $\mathrm{X}$ & & $\mathrm{X}$ & & $.86, .89$ \\
\hline & $\mathrm{X}$ & & & & $.93, .97$ \\
\hline & & $\mathrm{X}$ & & & $.91, .94$ \\
\hline & & $\mathrm{X}$ & $\mathrm{X}$ & & $.90, .96$ \\
\hline & & & $\mathrm{X}$ & & $.91, .98$ \\
\hline & & & & $\mathrm{X}$ & $.93, .95$ \\
\hline
\end{tabular}


Table 5-18. Summary of neural network predicted object depths.

\begin{tabular}{|c|c|c|c|c|c|c|c|}
\hline \multirow{2}{*}{$\begin{array}{l}\text { Target Description } \\
\text { (Object \# and type) }\end{array}$} & & \multirow{2}{*}{\begin{tabular}{|c|} 
True Depth \\
(feet) \\
\end{tabular}} & \multicolumn{3}{|c|}{ Neural Net Predicted [lepth } & \multicolumn{2}{|l|}{ Error (feet) } \\
\hline & Level & & Minimum & Maximum & Average & true-min. & true-ave. \\
\hline \# 6 (wooden box w/ metal) & 0 & 5.1 & 3.87 & 4.55 & 4.37 & 1.23 & 0.73 \\
\hline & 1 & 4.6 & 3.96 & 4.53 & 4.31 & 0.64 & 0.29 \\
\hline & 2 & 4.1 & 3.96 & 4.53 & 4.31 & 0.14 & -0.21 \\
\hline · & 3 & 3.6 & 2.18 & 4.38 & 3.73 & 1.42 & -0.13 \\
\hline & 4 & 3.1 & 1.93 & 3.43 & 2.84 & 1.17 & 0.26 \\
\hline & 5 & 2.6 & 1.48 & 3.3 & 2.48 & 1.12 & 0.12 \\
\hline & 6 & 2.1 & 1.3 & 2.78 & 1.99 & 0.8 & 0.11 \\
\hline
\end{tabular}

\begin{tabular}{|c|c|c|c|c|c|c|c|}
\hline \#7 (55 gal barrel W/ foam) & 0 & 5.2 & 4.38 & 4.54 & 4.45 & 0.82 & 0.75 \\
\hline & 1 & 4.7 & 4.29 & 4.44 & 4.38 & 0.41 & 0.32 \\
\hline & 2 & 4.2 & 4.29 & 4.46 & 4.38 & -0.09 & -0.18 \\
\hline & 3 & 3.7 & 3.77 & 4.08 & 3.9 & -0.07 & -0.2 \\
\hline & 4 & 3.2 & 2.9 & 2.49 & 2.93 & 0.3 & 0.27 \\
\hline & 5 & 2.7 & 2.79 & 2.92 & 2.86 & -0.09 & -0.16 \\
\hline & 6 & 2.2 & 1.6 & $2 . \$ 4$ & 1.88 & 0.6 & 0.32 \\
\hline \multirow[t]{6}{*}{ \#15 (4 on 4, 5-gal vertical pails) } & 0 & 3.7 & 3.44 & 3.64 & 3.56 & 0.26 & 0.14 \\
\hline & 1 & 3.2 & 2.93 & 3.18 & 3.06 & 0.27 & 0.14 \\
\hline & 2 & 2.7 & 2.26 & 2.76 & 2.57 & 0.44 & 0.13 \\
\hline & 3 & 2.2 & 1.73 & 2.45 & 1.98 & 0.47 & 0.22 \\
\hline & 4 & 1.7 & 1.34 & 2.95 & 1.49 & 0.36 & 0.21 \\
\hline & 5 & 1.2 & 1.29 & 2. 699 & 1.48 & -0.09 & -0.28 \\
\hline \multirow[t]{6}{*}{ \#16 (pyramid of 6,5 -gal pails) } & 0 & 3.4 & 3.08 & $3 . \$ 4$ & 3.21 & 0.32 & 0.19 \\
\hline & 1 & 2.9 & 2.84 & 2.58 & 2.92 & 0.06 & -0.02 \\
\hline & 2 & 2.4 & 2.16 & 2.76 & 2.64 & 0.24 & -0.24 \\
\hline & 3 & 1.9 & 1.71 & 2.53 & 1.96 & 0.19 & -0.06 \\
\hline & 4 & 1.4 & 1.37 & 2.2 .8 & 1.53 & 0.03 & -0.13 \\
\hline & 5 & 0.9 & 1.35 & 1.89 & 1.49 & -0.45 & -0.59 \\
\hline \multirow[t]{6}{*}{$\# 25$ (vertical iron pipe) } & 0 & 4.5 & & & & & \\
\hline & 1 & 4 & 3.73 & 3.13 & 3.75 & 0.27 & 0.25 \\
\hline & 2 & 3.5 & 2.78 & 2.83 & 2.81 & 0.72 & 0.69 \\
\hline & 3 & 3 & 2.78 & 2.83 & 2.8 & 0.22 & 0.2 \\
\hline & 4 & 2.5 & 2.79 & 2.81 & 2.8 & -0.29 & -0.3 \\
\hline & 5 & 2 & & & & & \\
\hline \multirow[t]{6}{*}{ \#26 (concrete w/ steel loop) } & 0 & 3.8 & 4.36 & 4.65 & 4.56 & -0.56 & -0.76 \\
\hline & 1 & 3.3 & 4.17 & 4.63 & 4.49 & -0.87 & -1.19 \\
\hline & 2 & 2.8 & 2.93 & 3.25 & 3.12 & -0.13 & -0.32 \\
\hline & 3 & 2.3 & 2.93 & 3.41 & 3.17 & -0.63 & -0.87 \\
\hline & 4 & 1.8 & 2.78 & 3.54 & 3.08 & -0.98 & -1.28 \\
\hline & 5 & 1.3 & 1.48 & 3.83 & 2.43 & -0.18 & -1.13 \\
\hline \multirow[t]{6}{*}{ \#28 (V8 truck engine) } & 0 & 4.9 & 4.18 & 4.47 & 4.34 & 0.72 & 0.56 \\
\hline & 1 & 4.4 & 3.87 & 4.45 & 4.21 & 0.53 & 0.19 \\
\hline & 2 & 3.9 & 2.83 & 3.07 & 2.96 & 1.07 & 0.94 \\
\hline & 3 & 3.4 & 2.81 & 3.13 & 2.95 & 0.59 & 0.45 \\
\hline & 4 & 2.9 & 2.78 & 3.15 & 2.92 & 0.12 & -0.02 \\
\hline & 5 & 2.4 & 1.44 & 3.4 & 2.48 & 0.96 & -0.08 \\
\hline
\end{tabular}


5.6.4.2 Depth Estimates. For input to the depth estimation networks, all those data points previously classified as target by the target versus background networks were used. Every data point within 12 objects was used for training. Selected results are listed in Table 5-18. Objects that were poorly classified tended to have worse depth estimates. Depths to objects on which we trained were usually within 5 to $10 \mathrm{~cm}$ ( 2 to $4 \mathrm{in}$.) of the actual depth. For test objects, the results were less accurate. We used data from one $x, y$ coordinate at a time for training and testing. Normally depth should be estimated by looking at the entire profile over an object. The neural network could estimate reasonable values for depth even if the accuracies were not as good as expected in a finished system. Another problem with the depth estimation was the lack of data. For most targets, only six different data collection levels (i.e., sensor-to-object depths) were available for training. The neural networks can produce more accurate depth estimates if a different training pattern is constructed and more in-depth information is available. An interesting result was how the network tested on object 9 and 10 from Experiment 3, which were stacked wooden boxes filled with different contents. The network tended to estimate an average depth between the two boxes but was slightly biased to the deeper depth of the metal-filled box. When the upper box was removed, the depth estimate to the lower box was greatly improved.

5.6.4.2 Conductivity Classification. We designed networks to classify each object as conductive or resistive. The criteria were that metallic objects were conductive and nonmetallic objects were resistive. A second output class was used for depth so the network would not be confused by changes in anomaly amplitude caused by burial depth. Objects greater than $3 \mathrm{ft}$ deep were classed as deep and objects shallower than $3 \mathrm{ft}$ were classed as shallow. The results are listed in Table 5-19. The networks can output real-valued numbers. Values near the limits of +1 or -1 can be considered to fall well inside the class boundaries; values near 0 can be considered to lie very near the class boundary but inside the correct class if the sign is correct. As with the depth estimate, there is a range in output depending on position within an object. Twenty-one of twenty-four targets were correctly classified as conductive or resistive using the average value of conductivity for all target data points. If the maximum values for conductive targets and the minimum value for resistive targets are included, only the PVC pipe is misclassified. The network classified the PVC pipe as strongly conductive.

Further work should be done to determine if moderately conductive or resistive targets can be classified as such. In an actual implementation of this network, it could be used at a level between the target versus background networks and the depth networks so that conductive or resistive targets could be fed to depth networks that only deal with such targets. Such a refinement may also improve the depth estimates.

\subsubsection{Conclusions}

This study was conducted to determine if neural networks could extract the same information that a trained geophysicist could from magnetic and EM data. One hundred and forty-five networks were trained and tested for purposes of target versus background classification, conductivity classification, and depth estimation. Optimizing the input patterns through preprocessing or editing the raw data were not considered. We feel the minimum capabilities of the networks have been presented and that with more study, better network results can be achieved. 
Table 5-19. Summary of neural network conductivity estimates.

\begin{tabular}{|c|c|c|c|c|c|c|c|c|c|}
\hline \multirow{2}{*}{$\begin{array}{l}\text { Target Description } \\
\text { (Object \# and type) }\end{array}$} & \multirow[b]{2}{*}{ Level } & \multicolumn{4}{|c|}{ True Depth Neural Net Predicted Depth } & Conductivity & \multicolumn{3}{|c|}{ Neural Net Prediction } \\
\hline & & (feet) & Minimum & Maximum & Average & conduptive $=+1$ : resistive $=-1$ & $\operatorname{Min}$ & Max & Average \\
\hline \# 6 (wooden box w/ metal) & 0 & 5.1 & -1.01 & -0.02 & -0.91 & & & & \\
\hline & 1 & 4.6 & -1.01 & -0.33 & -0.97 & & & & \\
\hline & 2 & 4.1 & -1.01 & -0.033 & -0.97 & & & & \\
\hline & 3 & 3.6 & -1.00 & 0.17 & -0.94 & 1 & -0.98 & 1.08 & 0.96 \\
\hline & 4 & 3.1 & -0.99 & 0.9 & -0.29 & & & & \\
\hline & 5 & 2.6 & -0.86 & 1.16 & 0.61 & & & & \\
\hline & 6 & 2.1 & -0.75 & 1.16 & 1.08 & & & & \\
\hline
\end{tabular}

\begin{tabular}{|c|c|c|c|c|c|c|c|c|c|}
\hline \#7 (55 gal barrel $W /$ foam) & 0 & 5.2 & 4.38 & 4.54 & 4.45 & & & & \\
\hline 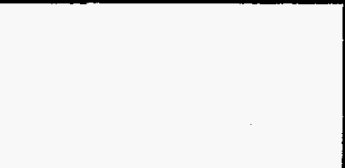 & $\begin{array}{l}1 \\
2 \\
3 \\
4 \\
5 \\
6\end{array}$ & $\begin{array}{l}4.7 \\
4.2 \\
3.7 \\
3.2 \\
2.7 \\
2.2\end{array}$ & $\begin{array}{l}4.29 \\
4.29 \\
3.77 \\
2.90 \\
2.79 \\
1.60\end{array}$ & $\begin{array}{l}4.44 \\
4.46 \\
4.08 \\
2.99 \\
2.92 \\
2.64\end{array}$ & $\begin{array}{l}4.38 \\
4.38 \\
3.90 \\
2.93 \\
2.86 \\
1.88\end{array}$ & 1 & 0.74 & 1.07 & 1 \\
\hline$\# 9$ (wooden box w/ wood \& paper) & $\begin{array}{l}0 \\
1 \\
2 \\
3\end{array}$ & $\begin{array}{l}2.8 \\
2.3 \\
1.8 \\
1.3\end{array}$ & $\begin{array}{l}-1.04 \\
-1.00 \\
-0.81 \\
-1.04\end{array}$ & $\begin{array}{l}0.93 \\
1.09 \\
1.18 \\
1.24\end{array}$ & $\begin{array}{l}0.09 \\
0.42 \\
0.64 \\
0.65\end{array}$ & -1 & -0.95 & 1.17 & 0.25 \\
\hline
\end{tabular}

\begin{tabular}{|c|c|c|c|c|c|c|c|c|c|}
\hline \multirow[t]{8}{*}{$\# 10$ (wooden box w/ferrous metals) } & 0 & 4.9 & -1.00 & 0.93 & 0.09 & \multirow{8}{*}{1} & \multirow{8}{*}{-1.19} & \multirow{8}{*}{1.17} & \multirow{8}{*}{0.41} \\
\hline & 1 & 4.4 & -1.00 & 1.09 & 0.42 & & & & \\
\hline & 2 & 3.9 & -0.81 & 1.18 & 0.64 & & & & \\
\hline & 3 & 3.4 & -1.04 & 1.24 & 0.65 & & & & \\
\hline & 4 & 2.9 & -1.04 & 1.15 & -0.01 & & & & \\
\hline & 5 & 2.4 & -1.03 & 1.24 & 0.61 & & & & \\
\hline & 6 & 1.9 & 0.26 & 1.24 & 0.91 & & & & \\
\hline & 7 & 1.4 & 0.43 & 1.24 & 0.95 & & & & \\
\hline
\end{tabular}

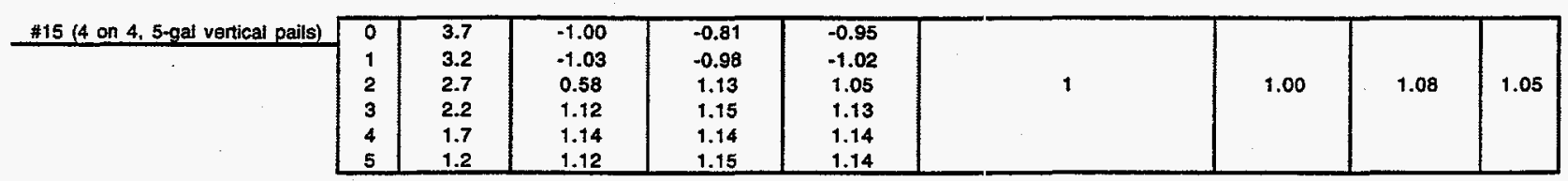

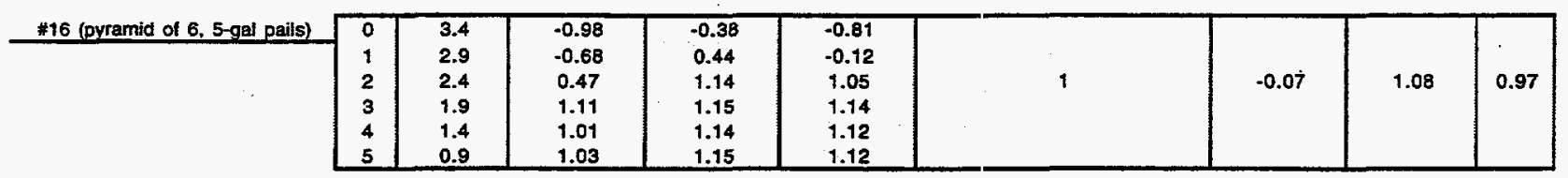

\begin{tabular}{|c|c|c|c|c|c|c|c|c|c|}
\hline \#17 (empty vertical 55-gal barrel) & 0 & 3.9 & -1.01 & -0.38 & -0.81 & & & & \\
\hline & $\begin{array}{l}1 \\
2 \\
3 \\
4 \\
5\end{array}$ & $\begin{array}{l}3.4 \\
2.9 \\
2.4 \\
1.9 \\
1.4\end{array}$ & $\begin{array}{c}-0.72 \\
-0.34 \\
0.84 \\
1.01 \\
1.05\end{array}$ & $\begin{array}{l}0.60 \\
0.96 \\
1.11 \\
1.13 \\
1.14\end{array}$ & $\begin{array}{c}-0.43 \\
0.54 \\
1.05 \\
1.05 \\
1.07\end{array}$ & 1 & -0.74 & 1.07 & 0.72 \\
\hline
\end{tabular}

\begin{tabular}{|c|c|c|c|c|c|c|c|c|c|}
\hline \#18 (horizontal empty 5-gal pail) & 0 & 4.8 & -0.91 & -0.60 & -0.84 & & & & \\
\hline & $\begin{array}{l}1 \\
2 \\
3 \\
4 \\
5\end{array}$ & $\begin{array}{l}4.3 \\
3.8 \\
3.3 \\
2.8 \\
2.3\end{array}$ & $\begin{array}{l}-0.94 \\
-0.86 \\
-0.70 \\
-0.17 \\
-0.14\end{array}$ & $\begin{array}{l}-0.44 \\
-0.07 \\
0.58 \\
0.38 \\
1.15\end{array}$ & $\begin{array}{l}-0.71 \\
-0.55 \\
0.09 \\
0.23 \\
0.69\end{array}$ & 1 & 0.95 & 1.08 & 1.00 \\
\hline
\end{tabular}

\begin{tabular}{|c|c|c|c|c|c|c|c|c|c|}
\hline \#19 (vertical einpty 5-gal pail) & \multirow[b]{2}{*}{$\begin{array}{l}0 \\
1 \\
2 \\
3 \\
4 \\
5\end{array}$} & \multirow[b]{2}{*}{$\begin{array}{l}4.9 \\
4.4 \\
3.9 \\
3.4 \\
2.9 \\
2.4\end{array}$} & \multirow[b]{2}{*}{$\begin{array}{l}-0.99 \\
-1.04 \\
-0.67 \\
0.88 \\
-0.89 \\
0.80 \\
\end{array}$} & \multirow[b]{2}{*}{$\begin{array}{c}-0.96 \\
-0.73 \\
0.91 \\
1.06 \\
1.00 \\
1.07\end{array}$} & \multirow[b]{2}{*}{$\begin{array}{c}-0.97 \\
-0.91 \\
0.28 \\
1.02 \\
0.36 \\
1.00\end{array}$} & \multirow[b]{2}{*}{1} & \multirow[b]{2}{*}{-1.00} & \multirow[b]{2}{*}{0.97} & \multirow[b]{2}{*}{1.00} \\
\hline & & & & & & & & & \\
\hline \multirow[t]{2}{*}{ \#20 (empty horizonal barrel) } & 0 & 3.1 & -0.19 & 1.15 & 0.91 & & & & \\
\hline & $\begin{array}{l}1 \\
2\end{array}$ & $\begin{array}{l}2.6 \\
2.1\end{array}$ & $\begin{array}{l}1.13 \\
1.06\end{array}$ & $\begin{array}{l}1.15 \\
1.14\end{array}$ & $\begin{array}{l}1.14 \\
1.13 \\
\end{array}$ & 1 & 0.72 & 1.07 & 1.04 \\
\hline
\end{tabular}


Table 5-19. (continued).

\begin{tabular}{|c|c|c|c|c|c|c|c|c|c|}
\hline \multirow{2}{*}{$\begin{array}{l}\text { Target Description } \\
\text { (Object \# and ype) }\end{array}$} & \multirow[b]{2}{*}{ Level } & \multirow{2}{*}{\begin{tabular}{|c|} 
True Depth \\
(feet) \\
\end{tabular}} & \multicolumn{3}{|c|}{ Neural Net Predicted Depth } & \multirow{2}{*}{$\begin{array}{c}\text { Conductivity } \\
\text { conductive }=+1 ; \text { resistive }=-1\end{array}$} & \multicolumn{3}{|c|}{ Neural Net Prediction } \\
\hline & & & Minimum & Maximum & Average & & $\operatorname{Min}$ & Max & Average \\
\hline \#21 (empty wooden box) & 0 & 3.6 & -1.01 & -0.97 & -0.99 & \multirow{6}{*}{-1} & \multirow{6}{*}{-1.04} & \multirow{6}{*}{0.96} & \multirow{6}{*}{.0 .92} \\
\hline & 1 & 3.1 & -1.01 & -0.64 & -0.88 & & & & \\
\hline & 2 & 2.6 & -0.89 & 1.01 & 0.07 & & & & \\
\hline & 3 & 2.1 & -0.46 & 1.06 & 0.89 & & & & \\
\hline & 4 & 1.6 & 0.46 & 1.08 & 0.96 & & & & \\
\hline & 5 & 1.1 & 1.42 & 1.15 & 0.96 & & & & \\
\hline \multirow[t]{6}{*}{ \#22 (wooden box w/dry dirt) } & 0 & 3.7 & -1.00 & .0 .52 & -0.92 & \multirow{6}{*}{-1} & \multirow{6}{*}{-1.03} & \multirow{6}{*}{-0.41} & \multirow{6}{*}{-0.97} \\
\hline & 1 & 3.2 & -0.84 & 0.77 & -0.26 & & & & \\
\hline & 2 & 2.7 & 0.68 & 1.06 & 0.95 & & & & \\
\hline & 3 & 2.2 & 1.04 & 1.07 & 1.06 & & & & \\
\hline & 4 & 1.7 & 0.74 & 1.08 & 1.01 & & & & \\
\hline & 5 & 1.2 & 0.41 & 1.09 & 0.99 & & & & \\
\hline \multirow[t]{6}{*}{ \#23 (wooden box w/wet dirt) } & 0 & 3.7 & -1.01 & -0.40 & -0.79 & \multirow{6}{*}{-1} & \multirow{6}{*}{-1.01} & \multirow{6}{*}{1.09} & \multirow{6}{*}{0.47} \\
\hline & 1 & 3.2 & -0.86 & 0.45 & -0.28 & & & & \\
\hline & 2 & 2.7 & 0.54 & 1.02 & 0.86 & & & & \\
\hline & 3 & 2.2 & 1.02 & 1.07 & 1.06 & & & & \\
\hline & 4 & 1.7 & 0.70 & 1.09 & 1.00 & & & & \\
\hline & 5 & 1.2 & -0.09 & 1.09 & 0.95 & & & & \\
\hline \multirow[t]{6}{*}{ \#24 (emply wooden box) } & 0 & 3.7 & -1.01 & -0.86 & -0.97 & \multirow{6}{*}{-1} & \multirow{6}{*}{-1.04} & \multirow{6}{*}{0.58} & \multirow{6}{*}{-0.91} \\
\hline & 1 & 3.2 & -0.98 & 0.46 & -0.61 & & & & \\
\hline & 2 & 2.7 & -0.66 & 1.02 & 0.45 & & & & \\
\hline & 3 & 2.2 & 0.93 & 1.07 & 1.04 & & & & \\
\hline & 4 & 1.7 & 0.90 & 1.08 & 1.04 & & & & \\
\hline & 5 & 1.2 & 0.70 & 1.09 & 1.03 & & & & \\
\hline
\end{tabular}

\begin{tabular}{|c|c|c|c|c|c|c|c|c|c|}
\hline \#25 (vertical iron pipe) & 0 & 4.5 & & & & & & & \\
\hline 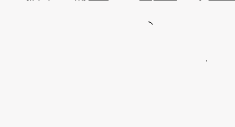 & $\begin{array}{l}1 \\
2 \\
3 \\
4 \\
5\end{array}$ & $\begin{array}{c}4 \\
3.5 \\
3 \\
2.5 \\
2\end{array}$ & $\begin{array}{l}-1.02 \\
-0.66 \\
-0.04 \\
1.12\end{array}$ & $\begin{array}{l}-1.02 \\
-0.29 \\
0.37 \\
1.14\end{array}$ & $\begin{array}{c}-1.02 \\
-0.46 \\
0.16 \\
1.13\end{array}$ & $\cdot$ & 1.00 & 1.07 & 1.02 \\
\hline
\end{tabular}

\begin{tabular}{|c|c|c|c|c|c|c|c|c|c|}
\hline \#26 (concrete w/ steel loop) & 0 & 3.8 & -1.03 & -0.99 & -1.02 & & & & \\
\hline & $\begin{array}{l}1 \\
2 \\
3 \\
4 \\
5\end{array}$ & $\begin{array}{l}3.3 \\
2.8 \\
2.3 \\
1.8 \\
1.3\end{array}$ & $\begin{array}{c}-1.08 \\
-0.18 \\
-0.16 \\
-0.8 \\
-0.98\end{array}$ & $\begin{array}{c}-1.01 \\
0.3 \\
0.29 \\
1.14 \\
1.15\end{array}$ & $\begin{array}{c}-1.02 \\
0.2 \\
0.11 \\
0.25 \\
0.84\end{array}$ & 1 & 0.12 & 1.08 & 1.00 \\
\hline
\end{tabular}

\begin{tabular}{|c|c|c|c|c|c|c|c|c|c|}
\hline \multirow[t]{2}{*}{ \#27 (vertical steel pipe) } & \multirow{2}{*}{$\begin{array}{l}0 \\
1 . \\
2\end{array}$} & \multirow{2}{*}{$\begin{array}{c}2.5 \\
2 \\
1.5 \\
\end{array}$} & \multirow{2}{*}{$\begin{array}{r}-1.04 \\
-1.04 \\
-1.04 \\
\end{array}$} & \multirow{2}{*}{$\begin{array}{r}-1.04 \\
-1.03 \\
-1.03 \\
\end{array}$} & \multirow{2}{*}{$\begin{array}{r}-1.04 \\
-1.09 \\
-1.04 \\
\end{array}$} & \multirow[b]{2}{*}{1} & \multirow[b]{2}{*}{1.00} & \multirow[b]{2}{*}{1.00} & \multirow[b]{2}{*}{1.00} \\
\hline & & & & & & & & & \\
\hline \multirow[t]{6}{*}{ \#28 (V8 truck engine) } & 0 & 4.9 & -1.03 & -1.00 & -1.02 & \multirow{6}{*}{1} & \multirow{6}{*}{0.14} & \multirow{6}{*}{1.08} & \multirow{6}{*}{1.01} \\
\hline & $1:$ & 4.4 & -1.03 & -0.91 & -1.01 & & & & \\
\hline & 2 & 3.9 & -0.95 & 0.22 & -0.33 & & & & \\
\hline & 3 & 3.4 & -0.95 & 0.27 & -0.39 & & & & \\
\hline & 4 & 2.9 & -0.15 & 1.15 & 1.06 & & & & \\
\hline & 5 & 2.4 & 0.07 & 1.15 & 0.98 & & & & \\
\hline \multirow{6}{*}{ \#29 (horizontal PVC pipe) } & 0 & 5,3 & -1.03 & -0.96 & -1.01 & \multirow{6}{*}{-1} & \multirow{6}{*}{0.98} & \multirow{6}{*}{1.08} & \multirow{6}{*}{1.00} \\
\hline & 1 & 4.8 & -1.03 & -0.96 & -1.01 & & & & \\
\hline & 2 & 4.3 & -0.09 & 0.27 & 0.20 & & & & \\
\hline & 3 & 3.8 & 0.02 & 0.25 & 0.13 & & & & \\
\hline & 4 & 3.3 & -0.72 & 0.25 & -0.27 & & & & \\
\hline & 5 & 2.8 & -0.71 & 0.48 & -0.08 & & & & \\
\hline \multirow[t]{6}{*}{ \#30 (horizontal steel pipe) } & 0 & 5.4 & -1.03 & -1.01 & -1.02 & \multirow{6}{*}{$t$} & \multirow{6}{*}{-0.89} & \multirow{6}{*}{1.08} & \multirow{6}{*}{0.96} \\
\hline & 1 & 4.9 & -1.03 & -1.02 & -1.02 & & & & \\
\hline & 2. & 4.4 & -0.08 & 0.21 & 0.05 & & & & \\
\hline & 3 & 3.9 & -0.61 & 0.14 & -0.18 & & & & \\
\hline & 4 & 3.4 & -0.85 & 0.15 & -0.45 & & & & \\
\hline & 5 & 2.9 & -0.49 & 0.88 & 0.27 & & & & \\
\hline
\end{tabular}


Table 5-19. (continued).

\begin{tabular}{|c|c|c|c|c|c|c|c|c|c|}
\hline \multirow{2}{*}{$\begin{array}{l}\text { Target Description } \\
\text { (Object \# and type) }\end{array}$} & \multicolumn{4}{|c|}{ True Depth (Neural Net Predicted Depth } & \multirow[b]{2}{*}{ Average } & \multirow{2}{*}{$\begin{array}{c}\text { Conductivity } \\
\text { Conductive }=+1 \text {; resistive }=-1\end{array}$} & \multicolumn{3}{|c|}{ Noural Net Prediction } \\
\hline & Level & (feet) & Minimum & Maximum & & & Min & $\operatorname{Max}$ & Average \\
\hline \#31 (horizontal copper bar) & 0 & 4.8 & -1.03 & 0.08 & -0.84 & & & & \\
\hline & 1 & 4.3 & -1.03 & 0.11 & -0.81 & & & & \\
\hline & 2 & 3.8 & -0.99 & 0.24 & -0.03 & 1 & -1.06 & 1.08 & 0.31 \\
\hline & 3 & 3.3 & -1.02 & 0.36 & -0.46 & & & & \\
\hline & 4 & 2.8 & -1.04 & 1.06 & -0.11 & & & & \\
\hline & 5 & 2.3 & -1.03 & 1.15 & 0.46 & & & & \\
\hline
\end{tabular}

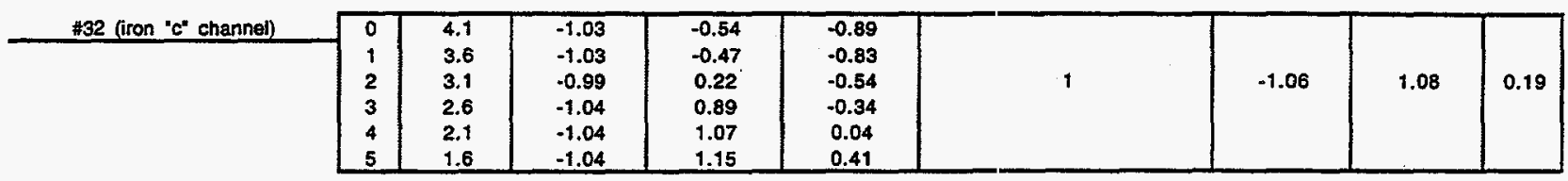

\begin{tabular}{|c|c|c|c|c|c|c|c|c|c|}
\hline \multirow[t]{6}{*}{ \#33 (horizontal aluminum bar) } & 0 & 5.2 & -1.03 & -0.63 & -0.88 & \multirow{5}{*}{1} & \multirow{6}{*}{-1.05} & \multirow{6}{*}{1.08} & \multirow{6}{*}{0.03} \\
\hline & 1 & 4.7 & -1.03 & -0.27 & -0.75 & & & & \\
\hline & 2 & 4.2 & -0.68 & 0.28 & -0.23 & & & & \\
\hline & 3 & 3.7 & -1.03 & 0.20 & -0.62 & & & & \\
\hline & 4 & 3.2 & -0.98 & 0.28 & -0.45 & & & & \\
\hline & 5 & 2.7 & -0.12 & 1.07 & 0.84 & & & & \\
\hline
\end{tabular}

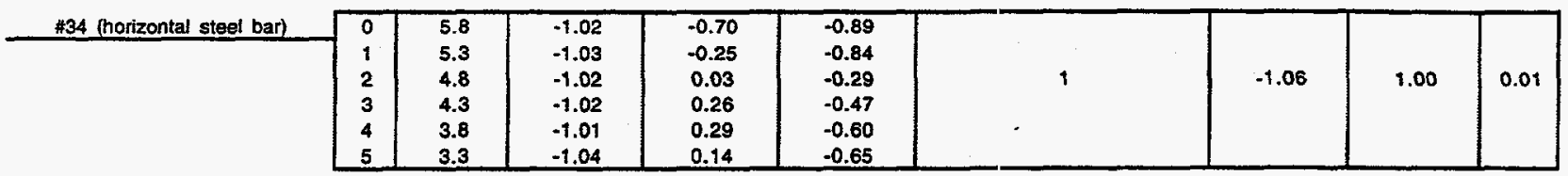


Research has established that target location and depth estimations for digface-type applications by neural networks is accurate and practical for the digface data available. The networks have quickly learned to recognize target locations accurately on over $85 \%$ of the digface data. Although the networks do not give a clear picture of size and dimension for the remaining $15 \%$ (horizontal objects of Experiment 6), they do recognize the location of an object in its proper place. After finding the targets, depth estimations have been reasonably successful. Experiment 1 was not useful because of the insufficient number of scan levels. Experiment 4 was not useful at all. The networks could not do anything with the chemical sensor data except recognize general locations of high chemical concentrations from the alcohol plumes. Depth estimations on Experiment 3 were complicated by the stacked objects and related signal interference. Another survey method such as GPR might help to solve this problem. Because of a lack of depth variability in the training data, deeper (e.g., 3-m down) objects with strong geophysical signatures may confuse the networks in a real world situation. To address this, more overall variability in the available data is needed.

The neural networks are capable of classifying the majority of the targets included in the data set given four EM components and two magnetic components. The results were more accurate as the sensors passed through level 3 of the data. Levels 0,1 , and 2 gave poor results. The best results were obtained when data from multiple levels were combined in the same training vector. For best results, both EM and magnetic data should be used in the same input vector although EM alone also gives acceptable results in most cases. If any of the four EM components were missing, the results were degraded when one EM was used for training. The results were not seriously impacted by missing EM data if magnetic data were also included in the input vector.

Metallic targets were the easiest to accurately map with the neural networks. Wooden boxes were located, but their shapes were ill-defined. PVC piping can be located by the EM sensor.

Even with the concerns mentioned, this study shows the usefulness of neural networks in applications such as the digface experiments. Future directions for this research should include a sensitivity study of the networks, refinements of the target versus background classification by examining the optimum ratio of target to background training samples, refinements of the conductivity classification and depth estimates, and preprocessing the data to produce more robust input pattern vectors. Incorporating the networks within a decision system to determine position, depth, and possible type of target should be examined. Methods of dealing with missing sensor data and conflicting sensor data need to be addressed.

\subsection{Application of Visual Data Base}

The integrated hardware and software platform described in Section 5.3 was applied to two visual data base problems. These were real world implementations of the functional design requirements and provided an opportunity to test the usability of the system in real scenarios. Several of the individuals polled early in the project expressed an interest in being able to visually inspect, manipulate, and query large data bases containing historical records of waste disposal areas. The two applications, the CTP Characterization Cell and Pit 9, were chosen because of availability of data and relevance to the program. 


\subsubsection{Cold Test Pit Descriptive Data Base}

The first fully integrated application of the Aurora Dataservel: and WTK virtual world environment was with the CTP. In this case, there was a good description of the subsurface objects and locations due to the construction of the Characterization Cell. This pit was carefully constructed so that the objects would be precisely known, both as to their location and composition (see Appendix A for a detailed description). A number of different objects were included to give a reasonable representation of expected conditions in a true waste site.

\subsubsection{Pit 9 Descriptive Data Base}

Information on the contents of the Pit 9 burial site came mostly from the Radioactive Waste Management Information System (RWMIS) data base (Litteer 1.988). The RWMIS data base was developed in 1971 to catalog all radioactive waste disposed of, or stored at the Radioactive Waste Management Complex (RWMC) as well as radioactive effluents generated at the INEL. While Pit 9 was accepting waste (mid-1967 to mid-1969), the information in RWMIS consisted of shipping summaries and disposal records. A sample shipping manifest is provided in Figure 5-32, which shows the amount of information available to build the Pit 9 portion of the RWMIS data base.

In general, the data base includes shipping and reporting dates, waste description [a generic description based on content codes developed by Clements (1982)], volume and weight data, radioactivity, and disposal location. The RWMIS data for Pit 9 can be divided into three separate categories:

1. Master data base has one entry for each waste shipment. Only general information on the shipment is contained in this data base.

2. Container data base has one entry for each container type on a shipment. There is potentially a one-to-many relationship between an entry in the master data base and entries in the container data base. The volume, location, weight, and content information is contained in this data base.

3. Nuclide data base has one entry for each nuclide type on a shipment (again a potential for one-to-many relationships). The nuclide information is fairly general (e.g., multiple fission products, multiple activation products).

The container data base was the most appropriate for developing the visual data base because it included separate volumes and content codes for different container types on the same shipment. However, this information is still general in nature, offering generic descriptions with approximate volumes and disposal locations.

A project lead by Smith et al. (1994) developed a comprehensive, detailed inventory of all contaminants and waste at the Subsurface Disposal Area (SDA) on the RWMC. As part of this task, the RWMIS data base was downloaded and verified against original shipping records. Discrepancies were noted and changes made in the RWMIS where allowed. These changes, along with discrepancies that were not incorporated into the RWMIS, were placed in a fourth dita base called the qualifier flag data base. This data base catalogs missing entries in the data base or shipping records, additions/deletions to 


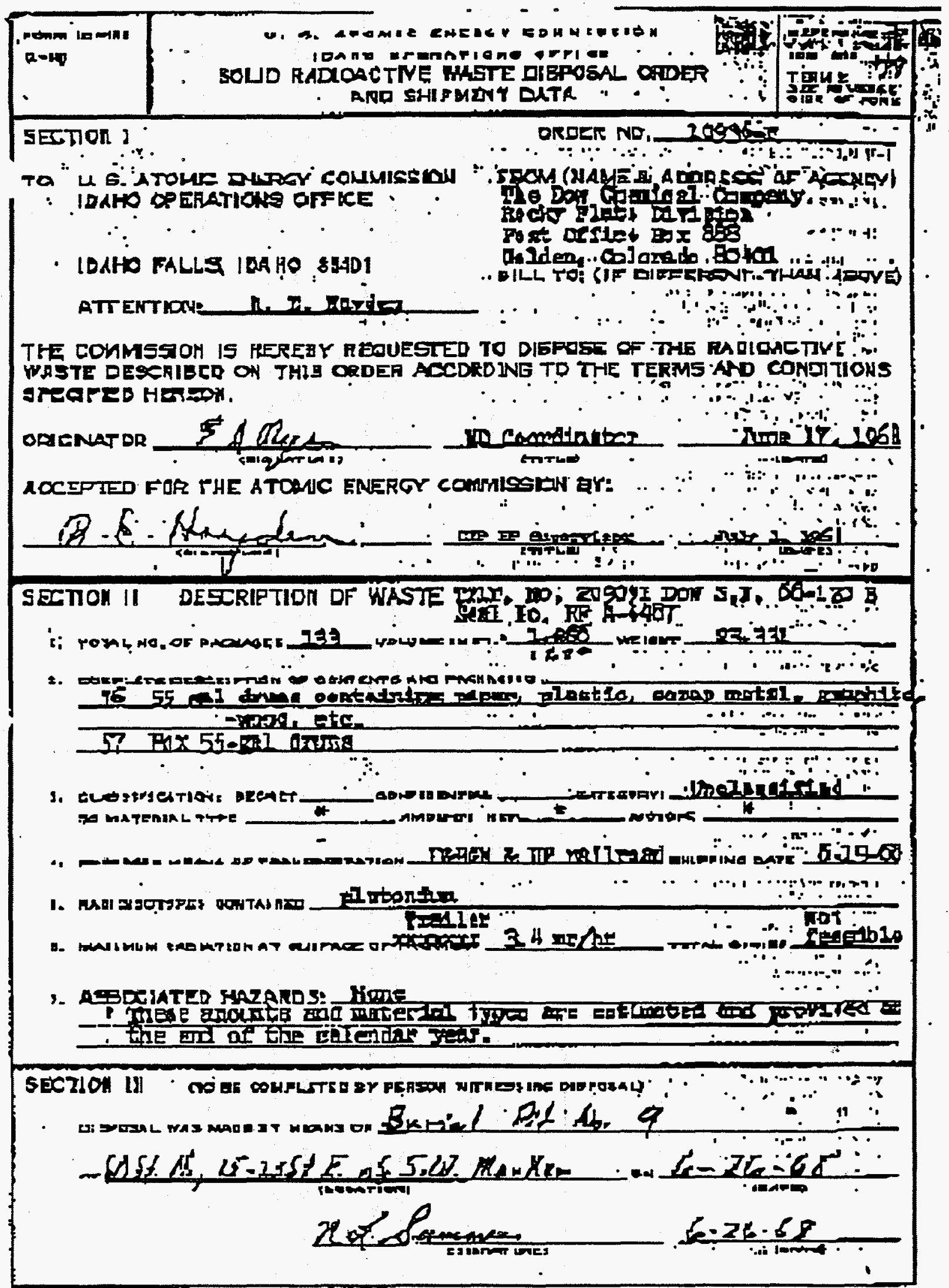

Figure 5-32. Sample shipping manifest for waste disposed of at Pit 9. 
the content codes, and more complete descriptions from the shipping records that were not entered into the RWMIS data base. The data base provides a more complete and accurate description of the waste contents but does not offer any more details on disposal location or waste volumes.

The product of this project was the Contaminant Inventory Data Base for Risk Assessment (CIDRA) and a four volume report cataloging all waste at the SDA. Unfortunately, it is not possible (without considerable labor) to tie the RWMIS container data base to the CIDRA data base and gain a better understanding of the Pit 9 waste. Through discussions with programmers who did much of the data base work for the CIDRA project, it was learned that the CIDRA data base is organized based on waste generator and not on disposal location. Key fields in the CIDRA data base that might directly correlate with those in RWMIS apparently do not exist.

Latest versions of the RWMIS data bases (i.e., master, container, and nuclide) and qualifier flag data base were obtained ${ }^{\mathrm{k}}$ for use in the visual data base. These daia bases include extra fields that were added during the CIDRA project for accounting purposes. In addition, several of the field titles had been changed from the original RWMIS data base. However, the content and integrity of the data remains the same as that from RWMIS. A list of fields and their descriptions for the master, container, nuclide, and qualifier data bases are shown in Appendix D. Complete listings of Pit 9 entries in these data bases are provided in Appendix E. Entries in each of these data bases for the sample manifest of Figure 5-32 are shown in Tables 5-20, 5-21, 5-22, and 5-23, respectively.

\subsubsection{Visual Data Base Implementation}

The initial visual data base application was designed using the Characterization Cell data and information. This application served as a prototype for future implementations such as the work based on data from Pit 9 at the INEL's RWMC. Work done on these two areas is discussed in this section.

For the burial sites discussed above, there a set (or series of sets) of scientific data that represents information such as magnetic field and magnetic gradient. These scientific data are viewed and analyzed using the AVS system. In addition to the scientific data, for each site there exists a collection of description data including information described in Sections 5.7.1 and 5.7.2. A primary goal (and advantage) of implementing a visual data base is that it enables useris to maintain all of the data (scientific and descriptional) in a centralized area promoting automation of data storage, analysis, display, and query.

Prior to integration of the system components, AVS and WTK were as established software supporting this project (AVS and WTK are described in detail in Section 5.3). However, it was necessary to find and integrate data base software that could fulfill the necessary requirements. For initial testing purposes, the information was placed originally in a free data base product called Postgres. However, as Postgres became unsupported, an extensive data base examination was conducted to define requirements and evaluate potential Relational Data Base Management Systems (RDMS) to support the project. Based on this effort, it was decided to use the Xidak Aurora Dataserver for this project.

k. Cathy Barnard, INEL, personal communication with Dave Harker, INEL, July 14, 1994. 
Table 5-20. Master data base entry for manifest in Figure 5-32.

\begin{tabular}{ll}
\hline BAD_HST & B \\
HSAREA & RFODOW \\
HSWASTE_TP & $\mathrm{S}$ \\
HSRADIO_AC & $\mathrm{R}$ \\
HSONOFF_SI & 1 \\
HSREPORT_D & $06 / 17 / 68$ \\
HSREPORT_T & 1010 \\
HSWASTE_DE & 30 \\
HSGROSS_VO & 35.4 \\
HSGROSS_WG & 10580000 \\
HSGROSS_AC & $6.1 \mathrm{E}-12$ \\
HDES_LOC & BGP09 \\
DOWNLOAD & 1 \\
VERIFIED & TRUE \\
TRU & TRUE \\
\hline
\end{tabular}


Table 5-21. Container data base entry $(1$ of 2$)$ for manifest in Fïgure 5-32.

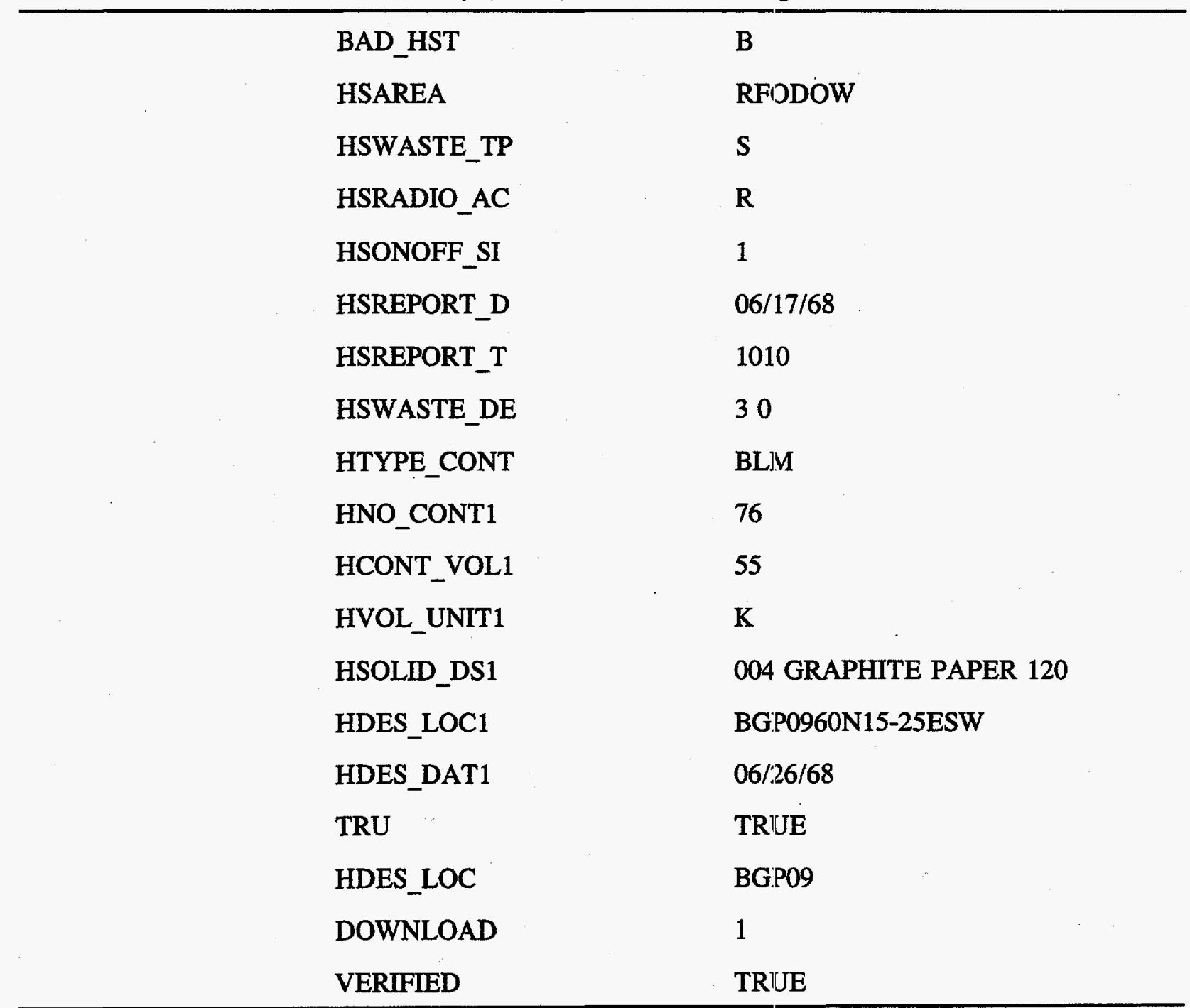


Table 5-22. Nuclide data base entry for manifest in Figure 5-32.

\begin{tabular}{ll}
\hline BAD_HST & B \\
HSAREA & RFODOW \\
HSWASTE_TP & S \\
HSRADIO_AC & R \\
HSONOFF_SI & 1 \\
HSREPORT_D & $06 / 17 / 68$ \\
HSREPORT_T & 1010 \\
HSWASTE_DE & 30 \\
HNUC_ID2 & PU-239 \\
HNUC_CON2 & $1 \mathrm{E}-10$ \\
HNUC_CUR2 & $6.1 \mathrm{E}-12$ \\
HNUC_NO2 & $<$ blank $>$ \\
HNUC_LES2 & $<$ blank $>$ \\
DOWNLOAD & 1 \\
HDES_LOC & BGP09 \\
VERIFIED & TRUE \\
TRU & TRUE \\
\hline
\end{tabular}


Table 5-23. Qualifier flag data base entry for manifest in Figure 5-32.

\begin{tabular}{|c|c|}
\hline SDA_LOC & P09 \\
\hline HSAREA & RFODOW \\
\hline HSWASTE_TP & $S$ \\
\hline HSRADIO_AC & $\mathbf{R}$ \\
\hline HSONOFF_SI & 1 \\
\hline HSREPORT_D & $06 / 17 / 68$ \\
\hline HSREPORT_T & 1010 \\
\hline HSWASTE_DE & 30 \\
\hline SHIP_MISS & FALSE \\
\hline ILLEGIBLE & $<$ blank $>$ \\
\hline SHIP_BLANK & CT2 CV2 CU2 GC NW1 NC1 \\
\hline ZERO_BLANK & $<$ blank $>$ \\
\hline CONTCODE 1 & 4+PLASTIC+SCRAP METAL \\
\hline CONTCODE2 & $<$ blank $>$ \\
\hline CONTCODE3 & $<$ blank $>$ \\
\hline CONTCODE4 & $<$ blank $>$ \\
\hline CONTCODE5 & $<$ blank $>$ \\
\hline CONTCODE6 & $<$ blank $>$ \\
\hline CONTCODE7 & $<$ blank $>$ \\
\hline CONTCODE8 & $<$ blank $>$ \\
\hline CONTCODE9 & $<$ blank $>$ \\
\hline CONTCODE10 & $<$ blank $>$ \\
\hline MISCELL & $\begin{array}{l}\text { \$UNIDENTIFIED PLUTONIUM; LISTED ON } \\
\text { RWMIS PRINT OUT AS PU-239 }\end{array}$ \\
\hline UPDATE - & TRUE \\
\hline KEY_FIELD - & FALSE \\
\hline OUD_KEY - & $<$ blank $>$ \\
\hline TRU - & TRUE \\
\hline
\end{tabular}


Significant determining factors in the decision to implement the Aurora Dataserver included

- Aurora fulfilled requirements established in the evaluation effort

- $\quad$ Aurora was code compatible with AVS and WTK

- Aurora was cost effective for our use

- Aurora was developed primarily as a Visualization Data Base Management System

- Xidak offered full support of product.

In June 1994, the Visualization and Imaging Team established the INEL as a Xidak Aurora Dataserver Beta Test Site. This allowed the project use of the software free of charge, which ensured close contact with and support from the Xidak company.

In obtaining the Aurora Dataserver, two different software capabilities were considered. We obtained the Aurora Dataserver, which is the data management system that stores and maintains the scientific data to be used by the analysis software and the Orion RDMS, which is the underlying backbone of Aurora. The description data are stored within an Orion RDMS. Aurora maintains information about the directories, data sets, and other assigned attributes in their respective name space in an Orion data base. It is this capability that enables both Aurora and Orion to respond to SQL queries.

The initial step in integration entailed entering the scientific data into the Aurora Dataserver and the description data into an Orion data base. To enter the scientific data into Aurora, it was necessary to develop a data-specific program that would create the data sets and insert the data into the dataserver. This program, originally developed by the Xidak Company, was written in C. This code was then edited for use in entering other data into the dataserver. Ultimately four programs were necessary to enter the CTP Characterization Cell data (gridded and unstructured) and Pit 9 data (gridded and unstructured).

Generation of the Orion data base involved creating the data base table and entering the data, all of which was done with a series of SQL commands. Scripts were written as was necessary to convert the data from its original format into a format that Orion (SQL) could understand. Such was the case for the Pit 9 information obtained from the RWMIS data base at the INEL. The description data for the CTP Characterization Cell was obtained from the final report of the digface characterization performance tests (Josten 1995). Once all of the data were entered, SQL queries were performed on the data base. For example, the SQL command select * from descr retrieves the information shown in Table 5-24 from the Characterization Cell data base.

To connect Aurora with AVS, a module was created specifically for this project by Xidak. Other necessary modules were created by editing the original code. The four modules created for this project allowed analysis of the CTP data (gridded and unstructured) and the Pit 9 data (gridded and unstructured). Appendix F provides some of this source code. 
Table 5-24. Data retrieved from Characterization Cell data base.

\begin{tabular}{|c|c|c|c|c|c|}
\hline 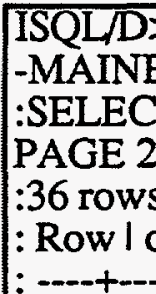 & $\begin{array}{l}\text { from descr } \\
\text { atch } \\
\text { d l experime } \\
\end{array}$ & nt I nff_ & $\begin{array}{c}\text {-- PAGE } \\
\vdots \\
\text { ne }|\operatorname{dim} 1| \operatorname{dim} 2 \mid \operatorname{dim} 3\end{array}$ & & h INW* \\
\hline$: 11$ & $1 \mid\{1\}$ & | ctp01 & INULLINULLINULLI & 2.9 & $1\left\{5^{*}\right.$ \\
\hline 21 & $21\{1\}$ & 1 ctp02 & |NULLINULLINULL| & 2.6 & $1\left\{5^{*}\right.$ \\
\hline 31 & $31\{1\}$ & 1 ctp03 & INULLINULLINULL| & 3 & $1\{1 *$ \\
\hline 41 & $4 !\{1,2\}$ & 1 ctp04 & $\begin{array}{lllllll}1 & 4 & 1 & 4 & 1 & 2 & 1\end{array}$ & 5.4 & $112 *$ \\
\hline 51 & $5 \mid\{1,2\}$ & 1 ctp05 & $\begin{array}{llllllll}1 & 4 & 1 & 4 & 1 & 2 & 1\end{array}$ & 5.1 & $1\{1 *$ \\
\hline 61 & $61\{2\}$ & 1 ctp06 & $\begin{array}{lllllll}1 & 4 & 1 & 4 & 1 & 2 & 1\end{array}$ & 5.1 & $1\{1 *$ \\
\hline $7 i$ & $71\{2\}$ & I ctp07 & INULLINULLINULLI & 5.2 & $1\{1 *$ \\
\hline 81 & $81\{2,3\}$ & 1 ctp08 & $\begin{array}{lllllll}1 & 4 & 1 & 4 & 2 & 1\end{array}$ & 4.8 & $1\{1 *$ \\
\hline 91 & $91\{3\}$ & I ctp09 & 14141. & 2.8 & $1\{3 *$ \\
\hline 101 & $10 \mid\{3\}$ & I ctp10 & \begin{tabular}{llllll|} 
& 4 & 4 & 2
\end{tabular} & 4.9 & $1\left\{3^{*}\right.$ \\
\hline $11 !$ & $11 \mid\{3\}$ & |ctp11 & INULLINULLINULL| & 5 & $1[1 *$ \\
\hline 121 & $12 \mid\{4\}$ & I ctp12 & INULLINULLINULL| & 2.4 & 1\{\}$^{*}$ \\
\hline 131 & $13 \mid\{4\}$ & I ctp13 & INULLINULLINULL| & 2.4 & 1\{\}$*$ \\
\hline 141 & $14 \backslash\{4\}$ & I ctp14 & INULLINULLINULL| & 2.4 & if\}* \\
\hline 151 & $15 \mid\{4,5\}$ & |ctp15 & INULLINULLINULL| & 3.7 & $1\{3 *$ \\
\hline 161 & $16 \mid\{5\}$ & I ctp16 & INULLINULLINULL| & 3.4 & $1\{3 *$ \\
\hline $17 i$ & $17 \mid\{5,6\}$ & |ctp17 & |NULLINULLINULL| & 4 & $1\left\{4^{*}\right.$ \\
\hline 181 & $18 \mid\{4,5\}$ & I ctp18 & |NULLINULLINULL| & 4.8 & 1\{\}$*$ \\
\hline 191 & $19 \mid\{5\}$ & | ctp19 & INULLINULLINULL| & 4.9 & $1\left\{7^{*}\right.$ \\
\hline 201 & $20 \mid\{5\}$ & 1 ctp20 & INULLINULLINULLI & 3.1 & I\{\}* \\
\hline $21 !$ & $21 \backslash\{4,5\}$ & I ctp21 & $\begin{array}{lllllll}1 & 2 & 4 & 4 & 2 & 1\end{array}$ & 3.6 & $199^{*}$ \\
\hline 221 & $221\{5\}$ & $1 \operatorname{ctp} 22$ & \begin{tabular}{ll|l|l|l} 
& 2 & 4 & 4 & 1
\end{tabular} & 3.7 & $\mid\left\{1^{*}\right.$ \\
\hline 231 & $23 \mid\{5\}$ & $1 \operatorname{ctp} 23$ & $\begin{array}{lllllll}1 & 2 & 1 & 4 & 1 & 2 & 1\end{array}$ & 3.7 & I\{1* \\
\hline 241 & $24 \mid\{5,6\}$ & $1 \operatorname{ctp} 24$ & $\begin{array}{lllllll} & 2 & 1 & 4 & 2 & 2\end{array}$ & 3.9 & $\left\{9^{*}\right.$ \\
\hline 251 & $25 \mid\{5,6\}$ & I ctp25 & INULLLINULLINÜLL| & 4.5 & 1\{\}$^{*}$ \\
\hline 261 & $26 \mid\{5,6\}$ & 1 ctp26 & INULLINULLINULL| & 3.8 & 1\{\}$^{*}$ \\
\hline 271 & $27 \mid\{6\}$ & $1 \operatorname{ctp} 27$ & INULLINULLINULL| & 2.5 & 1\{\}$*$ \\
\hline
\end{tabular}


These modules were developed by combining AVS and Aurora subroutines to allow the two programs to communicate with each other. When the AVS module is run, there is an edit option allowing the user to make an SQL query to the Aurora/Orion data base and the corresponding query response then appears in the AVS window environment allowing the user to work with that query specific data. For example, the user can make a query requesting all instances of file cabinet in the data base and the scientific data corresponding to any area of the burial site where a file cabinet can be found will be displayed.

To create the WTK/Aurora connection, it was necessary to embed Aurora code and subroutines into the WTK source code (see Appendix F). Specific options that were added to the WTK environment include the ability to make an SQL query directly to the Aurora/Orion data base from within WTK and view the corresponding query response. An option to load the Characterization Cell world and objects using information stored in the data base was added as well as the option to load by query, which enables the user to make a specific SQL query to Aurora/Orion. The objects that correspond to that query are then automatically loaded into the WTK environment. Queries corresponding to the Characterization Cell and Pit 9 can then be issued. Figure 5-33 shows the Characterization Cell world as viewed from the WTK environment. Code to load the Pit 9 environment is discussed below.

Another feature added to the WTK environment is the ability to retrieve information on specific objects in the world by using point and click. For example, while in WTK the user can select any object by using the mouse, the user can then select the object description, which automatically queries the data base and retrieves the information pertaining to the selected geometry. This is illustrated in Figure 5-34.

\subsubsection{Generating WTK Objects for Pit 9}

The boundaries of Pit 9 are defined by six monuments. The monument locations were obtained from an engineering design file ${ }^{1}$ that attempted to catalog all trench, pit, and soil vault locations for the SDA. These monument locations are listed in Table 5-25 and shown in Figure 5-35.

The locations are given in Idaho State Plane coordinates. Idaho has three state plane projections, western, central, and eastern. The SDA is located in the Idaho State Plane Eastern projection, with the origin located outside the valid area of the coordinate system. The $\mathrm{x}$ origin is at 112 degrees 10 minutes west longitude and the y origin is at 41 degrees 40 minutes north latitude (just south of the Idaho-Utah border, approximately due south of Idaho Falls). The coordinates at the origin are $x=500,000 \mathrm{ft}$ and $y=0 \mathrm{ft}$. This convention is used to ensure that there are no negative coordinates within the valid area of the projection. In the center of the coordinate system, the $\mathrm{x}$ axis is parallel to the equator and the $y$ axis points to true north. This is a cylindrical projection onto a flat surface, and there may be slight variations over an area the size of Pit 9 . These variations are approximately 1 minute or less and are ignored in the construction of the visual data base.

Because of uncertainties in the monument locations described by Yokuda, ${ }^{1}$ the actual locations have been assigned an error of $\pm 6 \mathrm{ft}$ when used to locate the actual pit boundary. Each waste disposal location given in the RWMIS data base references one of these six monuments.

1. E. L. Yokuda, "Locations of Pits, Trenches, and Soil Vault Rows," ERP-WAG7-05 Revision 2, EG\&G Idaho, Inc., July 1992. 


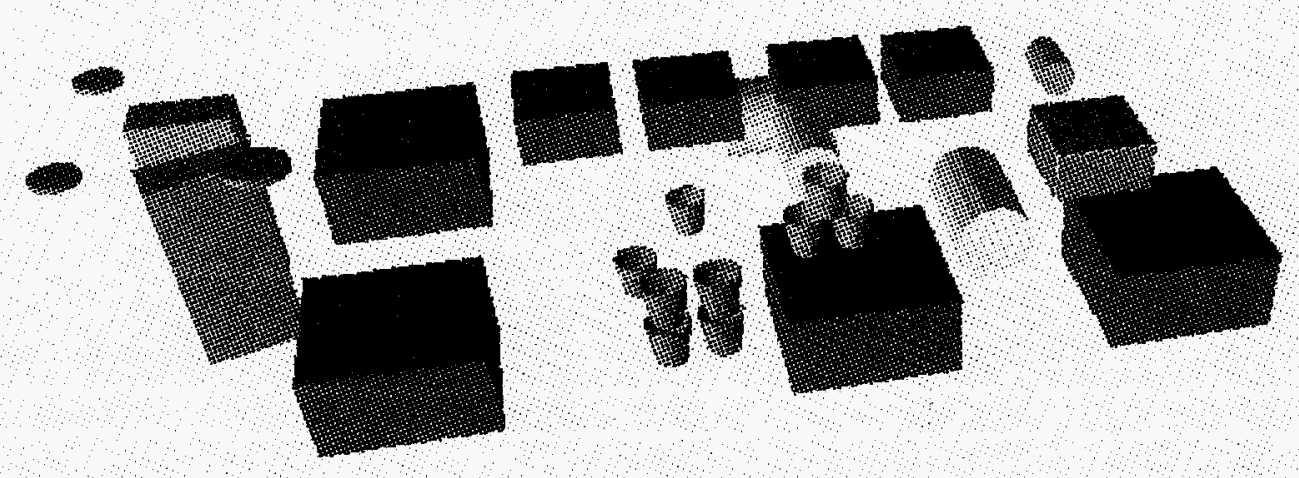

$\stackrel{\stackrel{1}{8}}{8}$

Figure 5-33. Characterization Cell objects in the WTK environment.

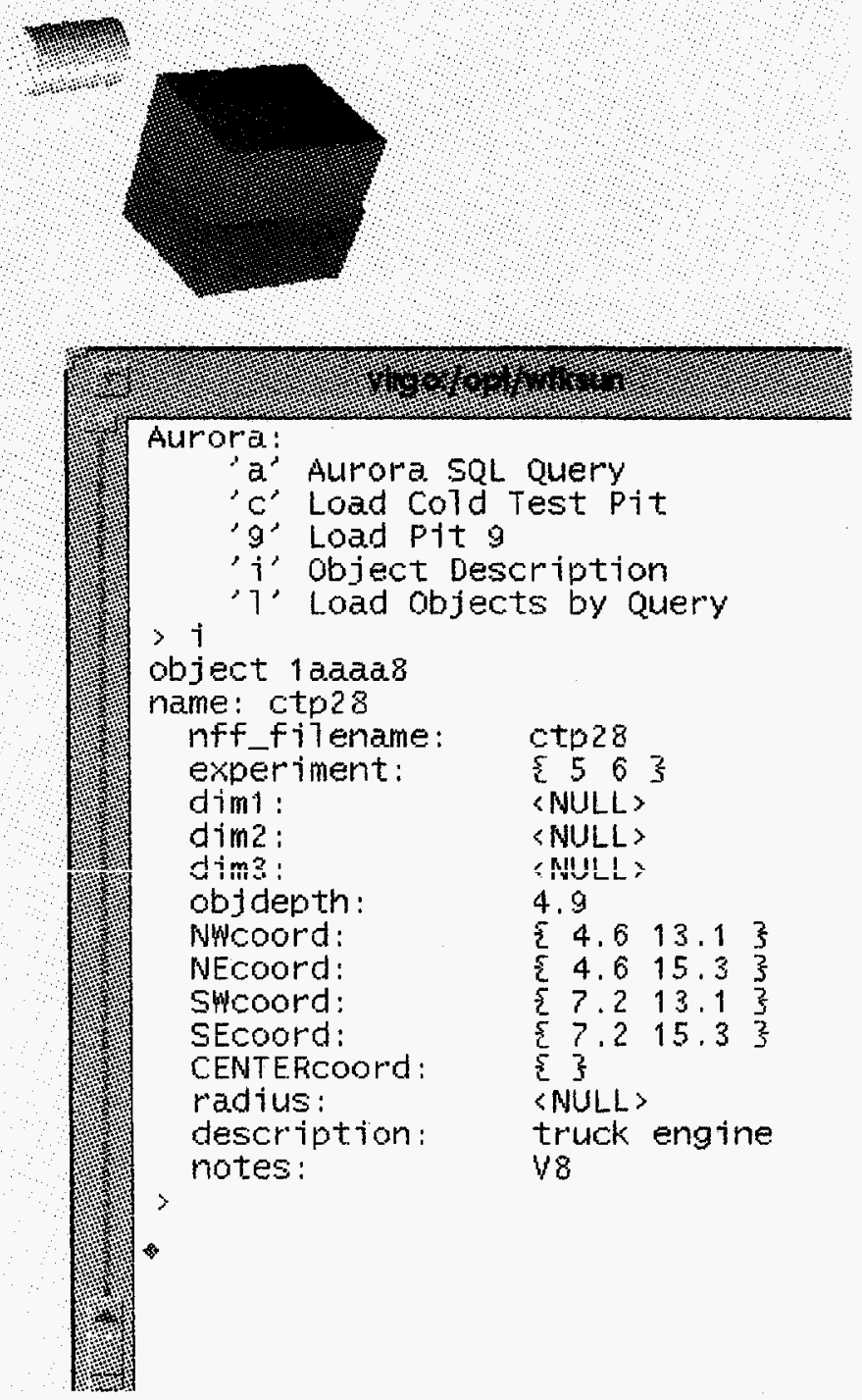




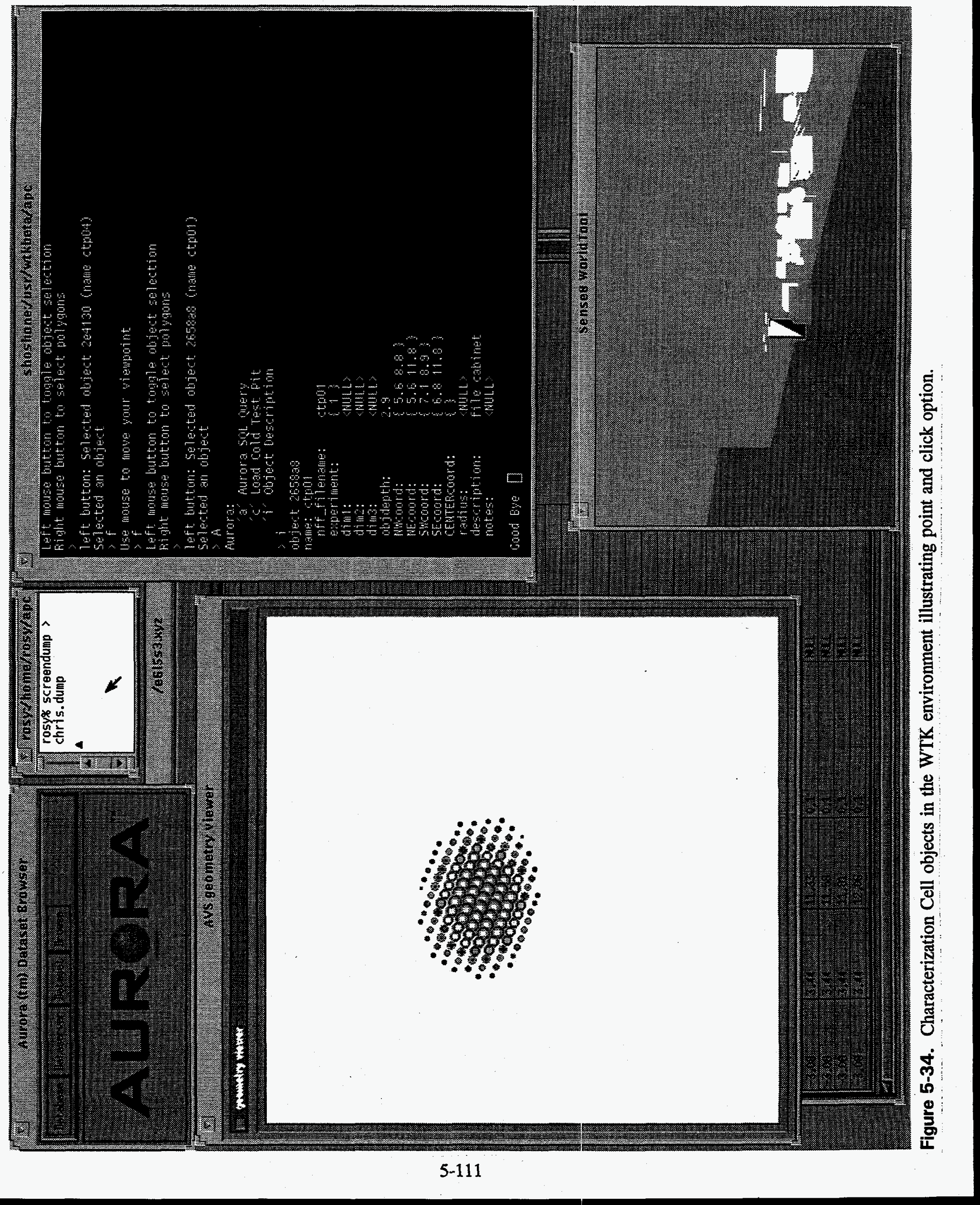


Table 5-25. Pit 9 monument locations.

\begin{tabular}{lll}
\hline Monument & Easting & Northing \\
\hline NW & 268295.18 & 669942.70 \\
NE & 268420.23 & 669933.40 \\
SE2 (E1) & 268303.71 & 669669.75 \\
SE3 (E2) & 268333.75 & 669662.91 \\
SE & 268266.31 & 669564.53 \\
SW & 268167.35 & 669556.98 \\
\hline
\end{tabular}

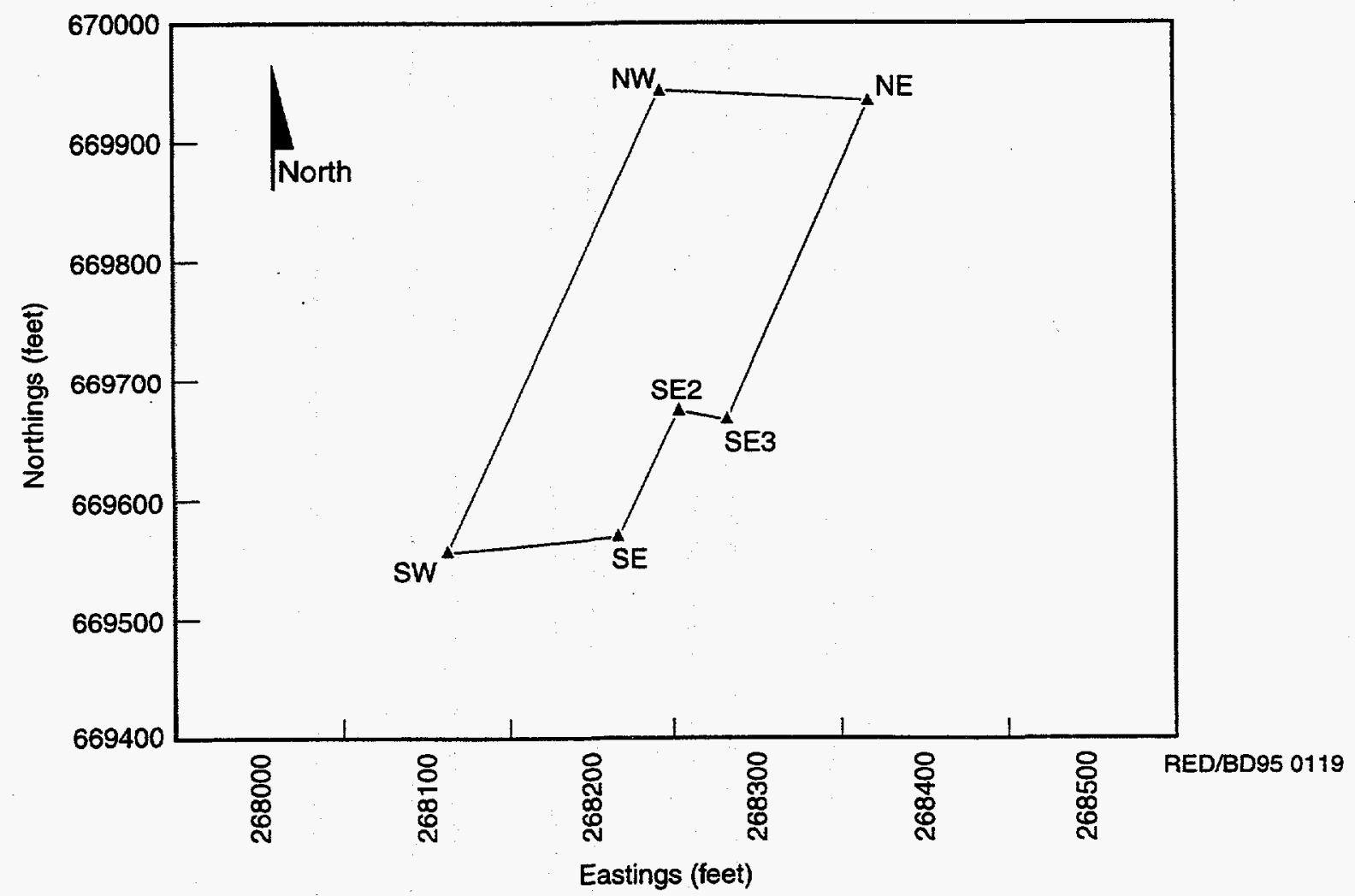

Figure 5-35. Pit 9 monument locations. 
A key to properly placing objects in the visual data base is to parse the location strings from the container data base. This string contains the reference monument as well as distance and bearing information from that monument. Generating ISP coordinates for the container location involves parsing the monument field and the two distance/bearing fields from the lcication string and offsetting the waste locations from the appropriate monument. When a range of distances is given for a certain bearing, a simple average is used to position the center of the waste. The source code that performs the parsing (and object sizing/placement) is given in Appendix G. A step-by-step example of parsing a location string is shown in Table 5-26.

The locations from the data base were first checked against the original shipping manifests with any significant discrepancies being noted. The waste locations were accurate (although sometimes vague) in all but a few cases. In these cases, a best guess is made by the parsing code. If no best guess can be made, the code output an error message and coordinates of $(0.0,0.0)$.

The coordinates for the waste locations were then plotted on a two-dimensional map, with the pit boundary overlaid on top. This revealed that a substantial number of calculated waste locations fell outside the pit boundary. The resulting plot is shown in Figure 5-36.

Fifty locations were manually checked, but no errors were found. The plot suggests that the operators filling out the shipping manifests may have assumed that the east and west sides of the pit were aligned in the north-south direction, and they made notes on the waste placement based on those assumptions. The east and west sides are actually parallel to a line approximately 18 degrees east of north. Resolution of this issue is key to providing an accurate model for the visual data base.

A difference between WTK objects in the CTP and Pit 9 objects is that while NFF files for each object in the CTP were generated and simply displayed in WTK, the objects in Pit 9 are created on the fly using utilities provided by WTK. Currently, the code parses the location string from the data base to place the object, determines the appropriate volume for the object (again from the data base), and generates a cube based on the calculated location and volume. All objects are placed at a depth of $14.2 \mathrm{ft}$, the average depth of the subsurface soils on the SDA (Meachum 1993). Figures 5-37 and 5-38 illustrate the WTK Pit 9 environment. Using information present in the data base, it would be possible to construct barrels (or any other objects for that matter) when appropriate. It would also be possible to color the objects based on waste generator or content codes. Useris may be able to provide insight into what would be useful. All the functionality of load by query and select and describe objects that was present for the CTP was also implemented for the Pit 9 application.

\subsubsection{Current Status and Future Directions}

Currently the AVS, WTK, and Aurora Datasever run on Sun and SGI Unix workstations. The AVS data analysis software also in available for most Unix platforms and for a number of nonUnix computers. The WTK software libraries from Sense8 are obtainable for all major workstations with dedicated threedimensional graphics hardware and PC compatibles under Windows and Windows NT. The present implementation has all software components executing on the same platform, in this case a Sun multiple processor workstation. Near-term plans call for the virtual world portion of the code to run on remote workstations and the data base to remain on the Sun server. Since data base accesses are relatively infrequent and of a burst nature, this should not be difficult or even introduce a noticeable delay, because the machines question are connected via a $100 \mathrm{Mb} / \mathrm{sec}$ FDDI ring. 
Table 5-26. Example of parsing a location string.

\begin{tabular}{cll}
\hline Step & \multicolumn{1}{c}{ Operation } & \multicolumn{1}{c}{ Result } \\
\hline 1 & Obtain the location string & BGP0960N15-25ESW \\
2 & Separate the SDA location & BGP09 60N15-25ESW \\
3 & Parse distance 1 and bearing 1 & BGP09 60N 15-25ESW \\
4 & $\begin{array}{l}\text { Parse distance 2 and bearing 2, leaving the monument } \\
\text { indicator }\end{array}$ & BGP09 60N 15-25E SW \\
5 & Strip off the bearings from the distances & BGP09 60 N 15-25 E SW \\
6 & $\begin{array}{l}\text { Determine whether there is a range (if so, average); also } \\
\text { determine whether to add or subtract the distance }\end{array}$ & BGP09 +60 N +20 E SW \\
7 & Retrieve the appropriate location for the monument & SW monument \\
& & $\begin{array}{l}\text { Easting }=268167.35 \\
\text { Northing }=669556.98\end{array}$ \\
8 & Add (or subtract) the distance from the monument & Waste location \\
& location & Easting $=\mathbf{2 6 8 1 8 7 . 3 5}$ \\
& & Northing $=\mathbf{6 6 9 6 1 6 . 9 8}$ \\
\hline
\end{tabular}

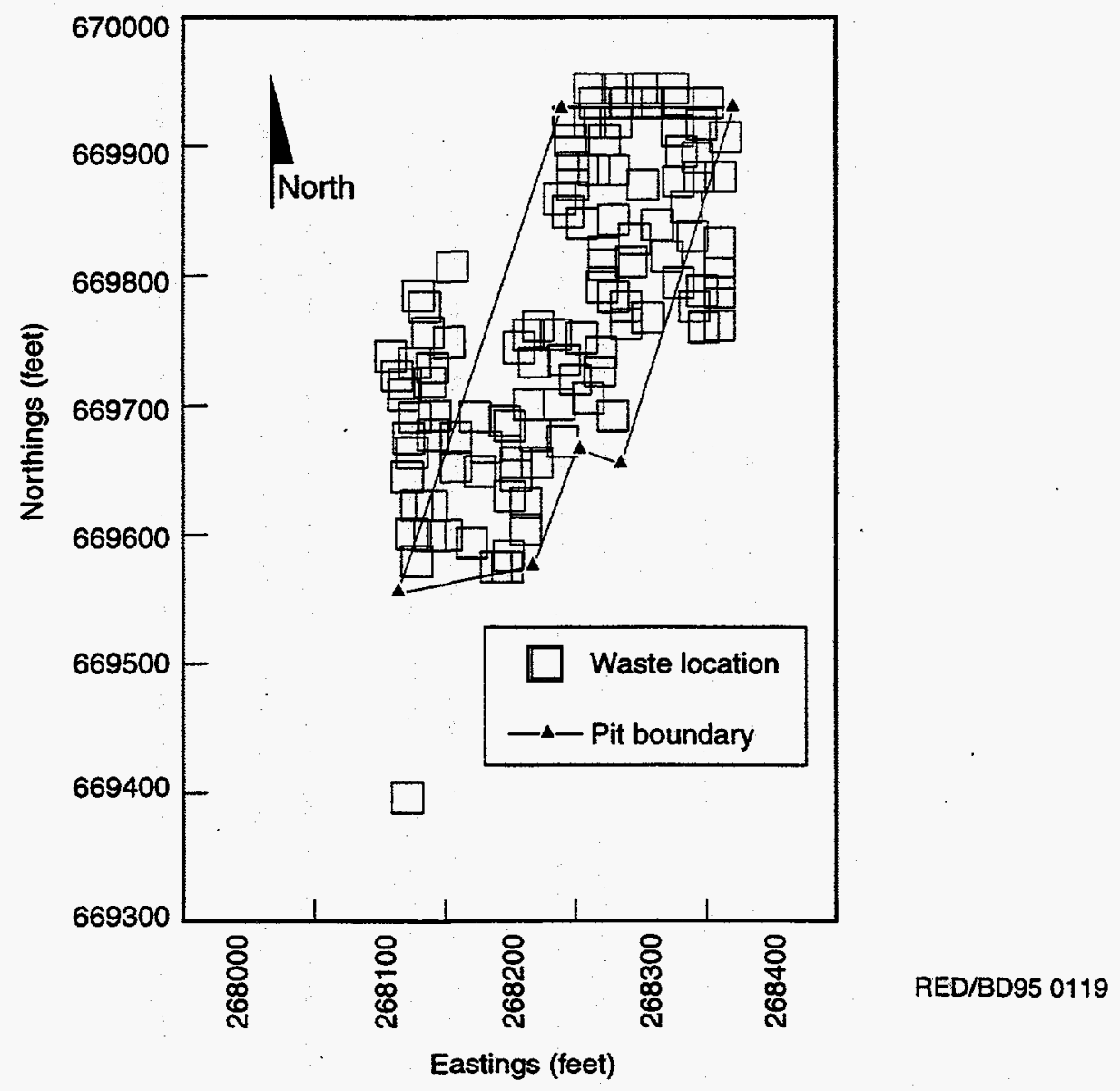

Figure 5-36. Pit 9 boundary locations and waste locations as reported in the shipping manifests. 


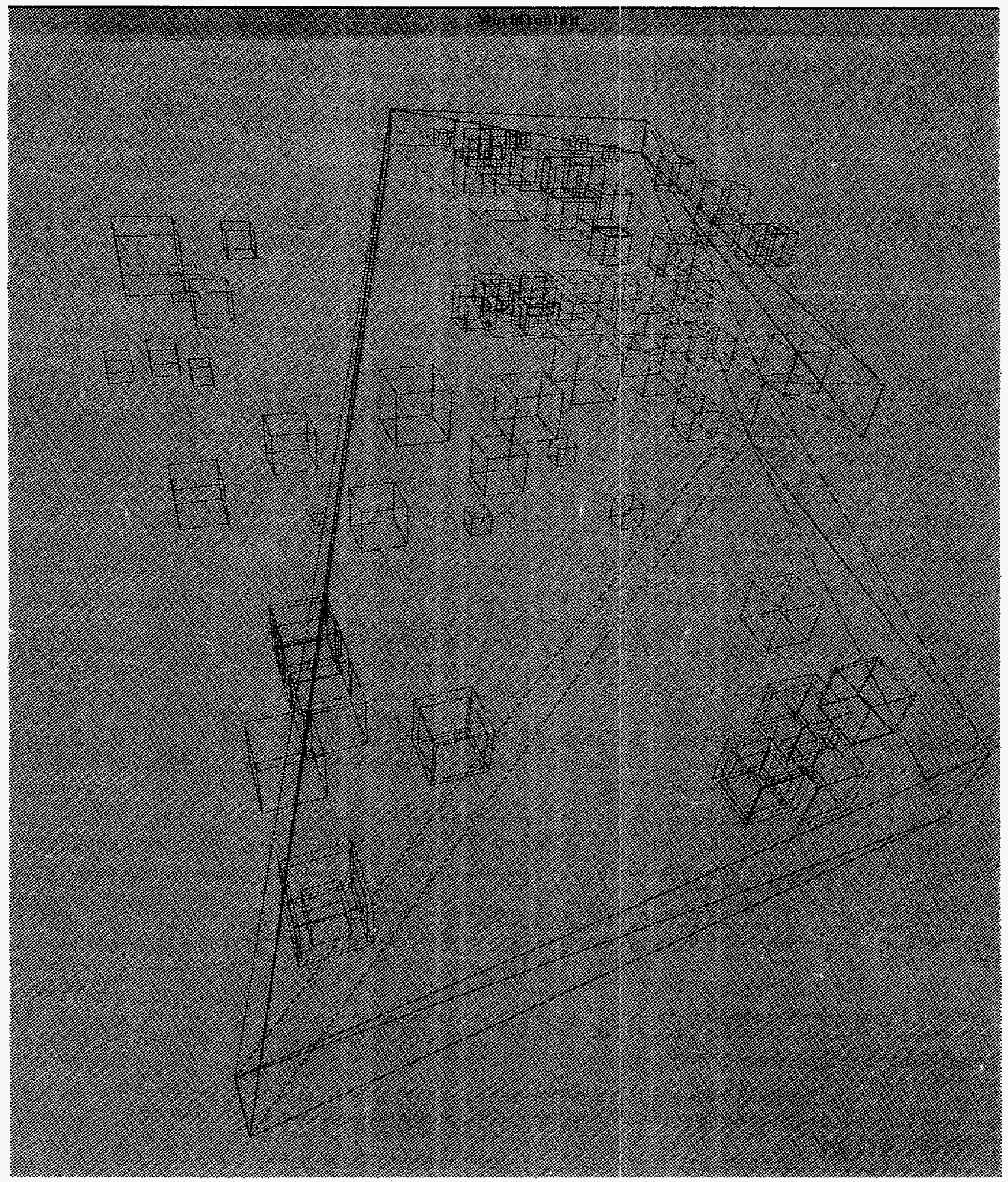

Figure 5-37. Illustration of the WTK Pit 9 world viewed from outside. 



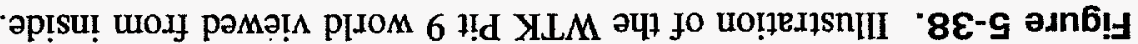

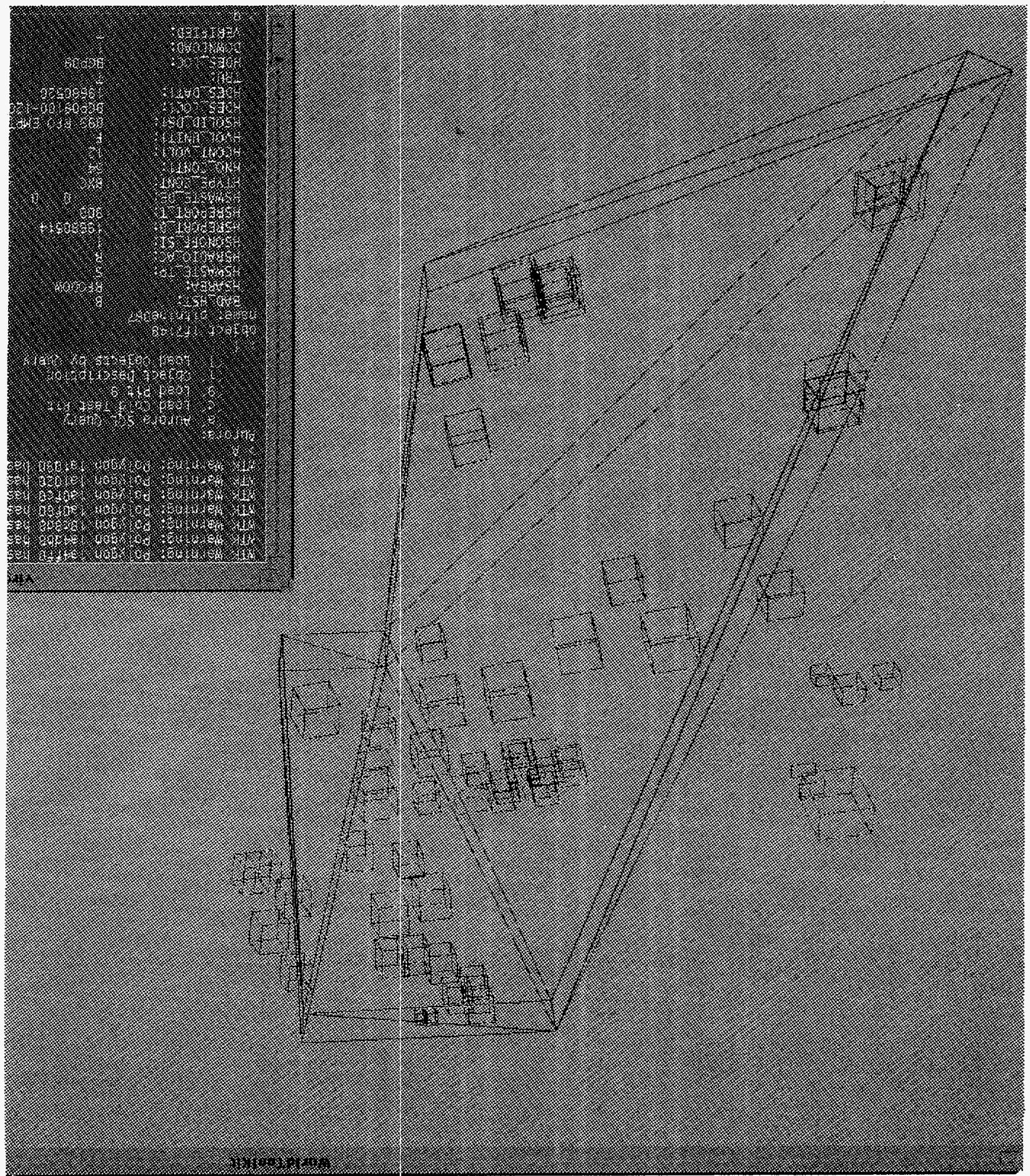



There is good possibility of migrating the software component's of the system to additional hardware platforms. The two graphical software packages, AVS and WTK, currently run on all platforms with sufficient performance to provide a realistic solution. These prodicts will soon be available on even a larger number of machines, providing accessibility across the entire spectrum of commonly used scientific workstations and high-end PCs. With the addition of IBM and Hewlett Packard support to the Evans \& Sutherland Freedom line of high-performance attached graphics processors, all major workstation vendors will have a hardware platform supported by these graphical software packages. With the increased capabilities of PC compatibles, including faster, nonsegmented processors, graphics hardware, and multiple processors, the PC will become a possible platform for some problems, especially in those environments where network resources make remote data bases a possibility.

The other commercial software component is the data base. This presently runs on most, but not all, Unix workstations. The major exception is the DEC Alpha, which Xidak has considered for porting their software but have declined to date due to lack of a perceived market. The PCs provide another potential market, but the difficulties involved in porting to that platform and the extremely small number of machines of that type with sufficient performance, make this a low probability occurrence. The best opportunities for PCs will probably remain in the networked environment where they can access the data base residing on the Unix servers.

\subsubsection{Conclusions and Recommendations}

The integrated software and hardware platform described in Section 5.3 has been used to develop prototype visual data bases for two applications. These include the CTP Characterization Cell and Pit 9. For the Characterization Cell, application data from the digface characterization activities (Josten 1995) were used in the development. For the Pit 9 application, historical data from the existing RWMIS data base and additional information generated during CIDRA activities was used.

From the development point of view, these applications showed the ability, benefit, and utility of having different sources of information about a particular waste site gathered into a centralized location. While analysis capabilities discussed in Section 5.3 have yet to be fully implemented into this environment, the potential benefit of such abilities is clear.

Although the Characterization Cell application was not particularly large (total of approximately 20 objects), the need to have computing capabilities with significant power was demonstrated. The visual data base implementation was run on several different classes of work stations. On lower end machines without dedicated graphics processors, the response to user movement through the objects was irritatingly slow as were screen updates and responses to queries. On newer machines (e.g., with dedicated graphics processors), the performance was much better and acceptable.

Development of the visual data base model of Pit 9 was a learning experience. While there appears to be an abundance of historical information for Pit 9, finding the most recent or most up-to-date information in a usable format was not straightforward. Also, the information in these data bases is only as good as the information from $20+$ year old shipping manifests. In spite of significant additional work such as described by Smith et al. (1994), it is clear that there will always be doubts about the accuracy of the information in the data base. As was shown in the previous section, use of the waste location information in the data base leads to objects being placed outside the pit boundaries in the model. The reason and explanation for this is an open issue at this point and may be related to our interpretation of 
the location information and accuracy of the location information. This issue is currently under investigation. It is concluded that the information must be used with caution, and human expertise and experience must be used in the process of building and using a visual data base.

\subsection{Evaluation of Immersion}

One of the main advantages of an immersive system is the ability to use the extremely high bandwidth communication system that comprises the human optical system. It is capable of transferring data at approximately $1 \mathrm{Gbit} / \mathrm{sec}$, which is two orders of magnitude greater than the fastest communication interface in most computer systems. By immersing the user in the virtual world, all of this bandwidth is available for communication with the computer. On traditional flat video monitors, only a small fraction of the information entering the optic nerve has originated in the computer application. There are many degrees of immersion varying from the minimal capabilities of a video monitor to the total immersion provided by stereo HMDs. For each application, many factors must be considered as to the appropriate degree of immersion necessary for efficient completion of the designated tasks.

Most immersive technologies include some mechanisms for the simulation of a three-dimensional representation of the virtual world. Stereo vision has several advantages over the monoscopic vision normally produced by computer displays. The first is the additional dimension of information display. Since the user gets the impression of depth, this can be used to convey another channel of information to the user. It is primarily used to give the sense of perspective, in which nearer objects appear larger than objects that are further away. This is the normal way in which humans view the real world and provides for a more intuitive interface into the computer-generated world.

Stereovision is obtainable in systems other than the fully immersive HMDs, by the use of glasses such as the CrystalEyes, a stereo ready monitor and appropriate software. In these cases, images for each eye is displayed on the screen and the switching of the glasses is controlled by software on the computer. In this fashion, each eye sees the correct image through the glasses, which is synchronized with the monitor. The brain receives an alternating left-eye/right-eye series of images. The frame switching occurs rapidly enough so that the brain integrates the pair into a perception of depth. Stereovision is almost always useful from viewing and manipulating objects in three-dimensional environments but usually has drawbacks in the price, performance, and resolution areas.

The RAMDACs used to generated the video signals have a fixed upper limit on the frequency at which their pixel clocks run, usually topping out at approximately $135 \mathrm{MHz}$. Since stereovision requires two images, a left and right eye, for each frame, there is already a twofold penalty to pay. One way to address this problem is to reduce the image resolution or the frequency in which the images are displayed. While this helps keep the pixel rates in the range achievable by the video circuits, the reduced image quality adversely affects the sense of immersion and overall usefulness of the system. If the spatial resolution is reduced too far, it becomes difficult to differentiate small objects, which is more important in a data analysis scenario than in an architectural walkthrough. The resolution and performance problems can be addressed by additional special purpose hardware, which tends to increase system cost dramatically.

One attribute of totally immersive systems is the freedom from peripheral distractions. This can be a benefit or a hindrance depending on the application. For some cases, total immersion not only focuses the user on the virtual world but can be critical to the success of the application. In training 
simulators, the user should as isolated as possible from the outside world, to better simulate the environment in which the user is operating. In these cases, HMDs and related technology such as caves provide a good solution. In other application areas, operators may want to interact with both their natural environments and VEs. In this case, the stereo glasses, augmented vision and see-through displays may be a better solution, since they allow the user a mixture of real and synthetic worlds.

The applications of VEs all have their own requirements on geometric and image fidelity, polygon rendering rates, use of texture maps, and acceptable sensor latency. These are controllable to some degree and result from compromises based on the overall requirements and resources. In all cases, there will be less than perfect sense of immersion. Currently it is too expensive to achieve perfection, so trade-offs are made with the parameters that go into constructing the system to achieve a reasonable level of realism control cost and time constraints.

Virtual worlds are constructed via polygonal surface representations. Little is done with direct volume renderings in VEs due to the extreme computational complexity of such renderings and the difficulty of mixing those with traditional polygon-based objects necessary in most virtual worlds. The objects are represented by a given number of polygons and using greater numbers of polygons results in a more accurate representation of the world. However, the rendering speeds of graphics hardware are measured by the number of polygons, usually triangle mesh strips, that can be rendered per second. By providing more accurate geometric descriptions of objects, the achievable frame rates fall accordingly. This is made more noticeable because most video hardware synchronizes screen redraws at an even multiple of the screen refresh rate. If a framebuffer has a refresh rate of $76 \mathrm{~Hz}$, it can redraw at 76,38 , or $19 \mathrm{~Hz}$. If a scene becomes even slightly too complex to render at $76 \mathrm{~Hz}$, it will immediately drop to $38 \mathrm{~Hz}$. Small increases in object complexity or number of objects can produce dramatic changes in the frame rates and resulting sense of immersion.

Another technique useful for maintaining good scene representation is the use of levels of detail (LOD) models. With this method different representations of geometries are stored, where each successive representation consists of a simplification of the previous. There are fewer polygons in each geometry of each LOD model, and with fewer polygons, the scene will render faster. Associated with each LOD model is a parameter, or set of parameters, that can be used to determine when that particular model is used. A common parameter is distance. When an object is further away, a less accurate geometry with fewer polygons can be used with no visual impact on the scene. Another set of useful parameters is linear and angular motion. If objects, or the viewpoint, are in motion, a less detailed model can easily provide the visual references needed during motion. Once motion ceases or decreases below a given threshold, the representation of an object can be changed to a more detailed model. This is particularly useful within large angular velocities where objects quickly cross the user's view. Extremely course models can be used, since they may only be within the user's view for factions of a second. Efficient use of this methodology requires fast access to many representations of multiple objects. The trade-off here is in the memory required to hold the different representations, and the code complexity to determine which to use. To date, the benefit seems to be worth the price.

Sensor latency is not as large of a problem in this application as in many other VEs. Sensor latency, as related to measurement of the position and orientation of the HMD, is directly related to how real the sense of immersion appears to the user. In this case, the sensor only needs to keep up with the rendering rate of the framebuffers. Due to the complexity of the scenes, the framebuffers are currently the system bottleneck. Estimations are made, this is callable predictive head tracking, where the user will 
be looking by the time the next frame gets rendered. This information can be used to select the view vector so that the image gets displayed to the users at the same time their heads are in the predicted position. Like all predictive algorithms, this can fail but has proven reliable and useful for the applications to date.

In some applications of VEs, texture maps are used to reduce the number of polygons needed to provide a realistic world. In these cases, an image of an object is mapped onto a small number of polygons so that the impression of a more complex object is provided. For data analysis applications, such as this project, this provides little if any benefit. There is not the need to provide extremely realistic impression of the virtual world. However, the details of precise position and shape of the objects are important. The system needs to be capable of providing high quality images with accurate display of relatively small objects to the user. If the trade-off is in terms of frame rates, this is acceptable as long as the induced latency does not cause disorientation or nausea. If the images lag the position sensors by too great an amount, the user can become lost because the cause and effect relationship of head motion and visual feedback has been disrupted.

Immersive displays do help communicate a more natural impression of the VE to the operator. This is true for all types of potential users, from heavy machinery operators, to geophysicists, to managers. The need for fully immersive displays, such as HMDs and Computer Audiovisual Environments (CAVEs), are probably required for a limited number of the tasks, such as training or operating via stereo cameras. To provide a genuine impression of operating within the waste environment in a reasonably authentic fashion, the totally immersive displays are necessary. Otherwise, the peripheral distractions alone will reduce the sense of immersion. For general data analysis, algorithm development and verification, system maintenance and introductory overviews, the stereo glasses or even monoscopic monitors work adequately and at a much lower cost. 


\section{INSIGHTS, ISSUES, PROBLEMS}

\subsection{Primary Issues}

\subsubsection{Unstructured Nature of the Digface}

It is likely that the majority of buried waste sites will be unstructured (similar to municipal landfills). The waste orientation will be arbitrary due to the nature in which the waste was placed, waste containers will have deteriorated, and isolated waste objects will bi the exception rather than the norm. There will be a large number of possible configurations due to supeiposition effects, natural effects (e.g., shifts in the waste placement, compaction), and degradation. For waste seam retrieval, implications of these considerations are significant.

Due to the expected large number of waste configurations, the waste signatures may be complex. Small differences in waste configuration may have substantial influences on the signature. Furthermore, as waste is removed, the configuration is changed and the signature will change. As such, pattern recognition schemes may have difficulty. If these schemes work reliably, this would be a great asset. It is expected that pattern recognition will be more appropriate during overburden removal. However, these techniques should not be relied on during retrieval. Once the waste seam is visible, the need for pattern recognition is less significant. Pattern recognition capabilities should be used if and when they are proven and become available. As such, the system should not depend on pattern recognition capabilities but rather should have the flexibility to use them if useful.

The unstructured and constantly changing topography of the digface also implies that robotic techniques based on a structured workspace model may not be useful for robotic control of equipment at the digface. A structured robotic workspace model may be useful for standard robotic applications such as those used in manufacturing, where the workspace does not often change, or the changes are small enough to be monitored. However, the digface topography is infinitely variable, and the effort to create models in such a rapidly changing environment is probably not worthwhile. This affects deployment of characterization sensors and also affects the overall organization of the remediation process, including digface characterization and information display and data interpretation.

Existing robotic approaches rely on a structured well-defined environment. Such an environment will never exist at the digface. The digface topography is unstructured and will always change as waste and material are removed. The most that can be accomplished is to build a model of the current topography, such as is planned in the digface project, using a laser range finder. However, the ability to build a model of the underground structure should not be assumed.

\subsubsection{Role of Human in the Remediation Process}

The presentation of characterization information needed to support retrieval operations is based on an overall view of the human role. The overall remediation system for hazardous sites contains some degree of automation and remote operation. In addition, the use of pattern recognition, deterministic algorithms, and advanced data display techniques is also based on a view of the human role in the process. The project work described in this report is based on the assumption that the human role is primary in both robotic and remote operations, and in data interpretation and the resulting decisions. 
None of the project work is designed or intended to support the integration of data interpretation directly with excavation. Integration is the role of the human operator. Some of the project work is designed to automate algorithms and display results for decisionmaking by geophysicists. (An example of this approach is the depth-to-object algorithms described in Section 5.4.) Other subtasks are designed to provide initial screening of data and display regions containing potential targets for review and verification by human operators. (Examples of this approach are the neural net and ATR techniques described in Sections 5.5 and 5.6). Subtasks have also been performed to provide an integrated environment for data integration and data display for use by the operators (geophysicists) in evaluating waste characteristics, specifically including hazards at the digface. In all cases, the VE techniques are intended to provide support to human operators (geophysicists) without distancing them from the primary sensor data, which are the direct link to the physical world.

\subsubsection{Overburden Removal and Waste Seam Retrieval}

Some fundamental differences exist between overburden removal and waste seam retrieval. Figures 6-1 and 6-2 (Josten and Roybal 1994) depict two modes of operation. During overburden removal, one major considerations is how far away from the waste forms the removal equipment is placed (e.g., what is the overburden depth). During a digface operation, a possible scenario is scanning the sensor suite over the digface in multiple passes to generate high resolution layered data from which general interpretations about waste form location and depth can be determined. During this characterization process (repeated sensor scanning and overburden removal), large amounts of data will be generated and an information overload problem may exist for analysis personnel. It is expected that during this process the VE will be of most use.

Once the waste seam is uncovered (the overburden has been removed), remotely-operated equipment using video equipment will view the waste forms being excavated. A major considerations at this point is locating hot (chemical and/or radioactive) spots. It is expected that before each retrieval pass, sensor scans consisting primarily of radiation and chemical detectors (or a thermal detector) will be performed to locate any hot spots. Hot spot evaluation using other detectors (active gamma measurements for example) may also be used if available. It is not obvious that significant geophysics sensor information is needed at this point, although this will depend on the circumstances of the situation. Essential display information would be an indication of the hot spot locations but the level of sophistication of the display (e.g., overlays, virtual world display) is not clear.

\subsubsection{Learning from Experience}

The integration of digface characterization with the excavation process provides conceptually for the ability to learn from experience (Josten and Roybal 1994) This is because knowledge of characterization sensor patterns gained from previous excavations could conceivably be applied during subsequent excavations. The pattern recognition work described in this report shows feasible application two techniques (ATR and neural networks) that are based on training and learning.

Additionally, the ability to do automated scans raises the possibility of obtaining data from more than one level above a given digface configuration and thus using multiple level data in the interpretation process. Examples were shown in the depth-to-object algorithm (Section 5.4.2) the neural net technique (Section 5.6), and another potential application was identified for automated target recognition (Section 5.5.6). 


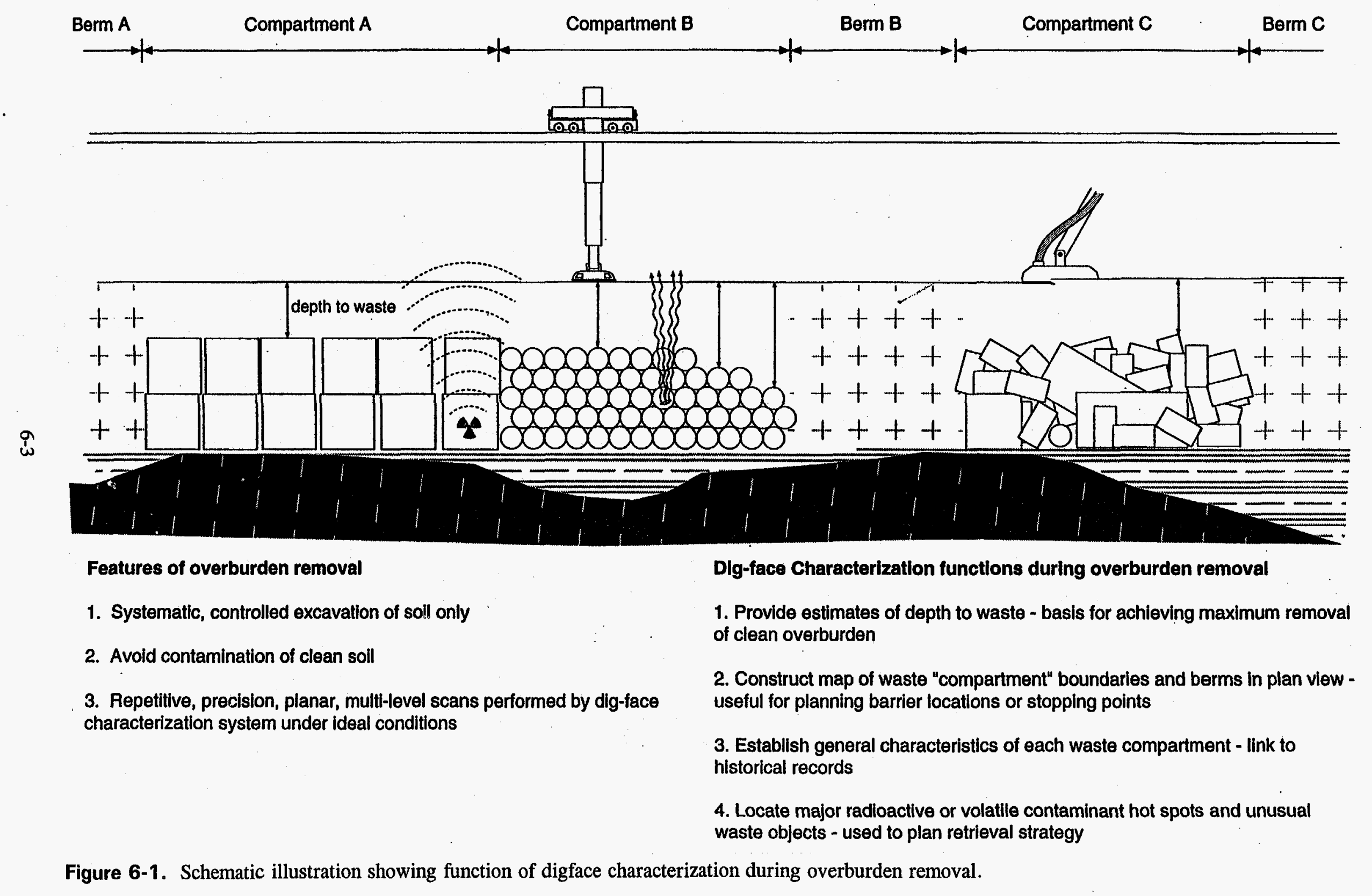




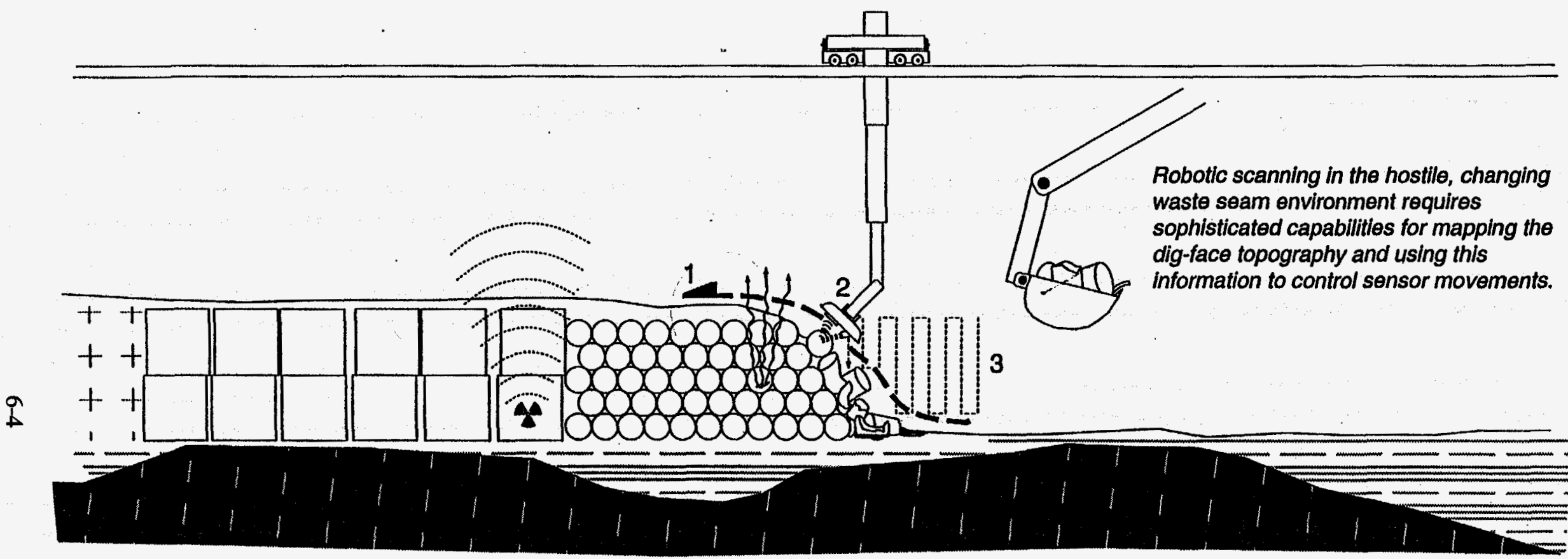

\section{Features of waste seam excavation}

1. Heavy construction style digging in hostile, uncontrolled environment of the waste seam

2. Assume all recovered material will require assay, but only a portion will require expensive processing or treatment

3. Waste seam environment changes constantly

\section{Dig-face characterization functions during waste seam excavation}

1. Routine scanning to monitor radioactive and volatile contaminant hot spots

2. In-depth in situ assay of exposed suspicious containers

3. Occasional systematic geophysical surveys to locate important objects such as reactor cores, vehicles etc.

Figure 6-2. Schematic illustration showing function of digface characterization during waste seam retrieval. 
Once the waste seam has been uncovered, the availability of multiple layers of data should not be relied on. This stems from several considerations. The problem changes as the digface changes and waste forms are removed. In most cases, data previously recorded at different levels will not necessarily apply (this depends on the waste type removed and the measurement type). Also, due to time constraints, it is unlikely that multiple layers of data will be recorded (or re-recorded) every time the digface changes. It is possible that two levels of data can be obtained during a single sweep by deploying multiple sensors. This will clearly be situation dependent, but total reliance should not be placed on interpretation techniques that require multiple levels of data. If multiple levels of data are available, full advantage should be taken of this fact.

An additional consideration for using information from previous sites in interpreting data obtained during later remediations is the size of the data and information files that would have to be dealt with in realistic situations. These would almost certainly be immense. The ability to archive information, while maintaining reasonable access to it for pattern matching, either automated or human, is a major consideration and requires development and demonstration.

A related issue for matching patterns to waste configurations is how to describe the waste configuration. One of the advantages of the digface characterization approach is the ability to continually match sensors data with the correct answer, where the correct answer is the recording of how the waste was actually configured in the pit. The answer is obtained during the excavation. This raises the issue of how the correct answer is going to be recorded, archived in a data base, and matched to the sensor data. The problem is compounded by the fact that the correct answer cannot be obtained because the excavation equipment destructively removes the waste from the pit as intended. Despite these problems, it is likely worth the effort to record information about the waste as it is excavated. The categories of information (e.g., visual description, videotape record, masses, classifications, hazards), detail of information, and how the information should be electronically stored are issues requiring substantial thought and effort to implement. Significant development and demonstration in this area is needed.

\subsubsection{Operator Qualification Requirements}

Various views exist regarding the anticipated end use of the information to be presented using VE or other advanced data interpretation and display techniques. In theory, the best situation would be to infallibly and usefully present the information in a manner immediately understandable by anyone involved in the remedial operations. Concepts like this have been discussed presenting the buried objects as they exist in "transparent" earth with different hazards displayed by techniques like color-coding. Unfortunately, there is little reason to believe that this will be feasible. Evidence so far supports the opposite belief.

The project approach has focused on supporting a technically sophisticated user of the data and information. This person is assumed to be a scientist who is familiar with the sensors being deployed. This scientist is assumed to be onsite during all excavation and digface characterization operations to provide input to the human operators of the remote excavation equipment. This approach is consistent with the overall role of the human operators described above in Section 6.1.2.

Based this assumption and the feedback obtained from the project rapid prototype (see Section 5.1.4), the desired functionality of a prototype environment incorporates features that primarily are intended to be used by a technically sophisticated user, such as a geophysicist (see Section 5.2). As 
currently envisioned, the system will likely not directly support a user such as an excavator operator without oversight and input from a support scientist.

\subsection{Secondary Issues}

\subsubsection{Stereovision and Virtual Environments (Mixing)}

Stereo/video has been proposed for examination and potential implementation during buried waste excavation. The proposers suggest that the use of stereo/video will provide excavation operations personnel an improved depth perception of the digface terrain and waste objects at the digface. One concept is to overlay stereo/video images with data presentations and/or objects rendered based on the data interpretation to provide the operations and analysts personnel with a combination of real and artificial images. The preferred method of geophysics data display may be some level of abstraction or inference based on the data and may not be a representation of any recognizable object. Given this consideration, it is not clear how useful overlaying stereo/video might be. At this point, the form of the virtual display cannot be assumed to necessarily relate to stereo/video images of the digface. The utility of overlays is not known. Therefore, at present, the ability to mix stereo/video and virtual images in the waste seam should not be relied on. There is currently no indication that an unstructured waste seam. will allow one to build up virtual objects of use. More than likely, the data and analysis results displayed in some abstract manner (relative to physical objects) will be more useful and possible. Stereo/video may be a benefit to operations, but overlays will require continuous geophysics support. As discussed above, it may not be necessary or sensible to provide continuous geophysical sensor data once in the waste seam. Under these conditions, it is not clear what will be mixed with stereo/video.

\subsubsection{Forward Modeling of Sensors}

Forward modeling of sensor response will be a difficult problem because the unknown configuration and properties of the waste. It is expected that this kind of modeling will be time consuming. Such models will require boundary and initial conditions. If the surrounding material properties are unknown (more or less), then many simulations may be required to find a solution that fits the present problem. This is akin to "given a unique measured response, what is the situation" rather than "given a situation, what is the unique response," and these are two very different problems. The project is not currently working on this problem. Mechanical Technologies, Inc. is, however, proposing some work in this area. As was discussed above with respect to pattern recognition, forward modeling simulation capabilities should be used if and when such methods become available and are proven but should not be relied on for the present. Again the system should not rely on the availability of such techniques but should not preclude their use either. 


\section{FULL-SCALE IMPLEMENTATION, RIELATED EFFORTS, AND COMMERCIALIZATION}

This section describes the issues and factors affecting full-scale implementation of VE techniques and software for the buried waste characterization application. The implementation of a VE system may benefit from, and in turn benefit, ongoing efforts in related areas. Some of these efforts are described. Additionally, commercialization of the project software and tools depends on both technical and market considerations. An overview of market potential and commercialization factors is presented.

\subsection{Full-Scale Implementation}

To assess full-scale implementation issues, a structured set of issues was prepared to use in evaluating each BWID technology (Bonnenberg 1994). The following items address each of the structured items for the VE technology.

1. Estimate the expected, useful life, and the operation and maintenance cost for the life cycle of the technology demonstrated. Operation and maintenance cost includes storage and disposal of primary and secondary wastestreams, operation of equipment (e.g., labor, electrical power, types of support equipment) and maintenance (e.g., cost of replacement parts, labor), and site restoration and decommissioning. Useful life should also include consideration for phased and expected technology obsolescence.

a. Because the technology is primarily techniques and knowledge embodied in software, it is expected that the useful life is not limited. The system capabilities would be expected to improve as time progresses, and the tools could be used as long as remediations are taking place. Over this time frame, estimated at 30 years, hardware and software changes would likely occur necessitating upgrades to the system. These could not reasonably be expected to exceed $\$ 100 \mathrm{~K}$ for each system. A separate system would be required for each remediation that was scheduled to occur simultaneously.

b. Production use of the VE tools is expected to take place as a piece of the overall retrieval and digface characterization control system. The VE subsystem assumes that basic infrastructure will be present, such as a control trailer with power and lighting. Hardware and software costs for the VE subsystem are estimated to be in the range of $\$ 100$ to $\$ 200 \mathrm{~K}$. Use would likely be by one or: more geophysicists comprising the technical support team for digface characterization.

c. Improvement of the system should result in adding new capabilities and incorporating knowledge gained in one excavation so that it is available for subsequent use. The amount of effort required for such improvement is difficult to estimate but probably could total up to an additional $20 \%$ of the excavation time, which represents a substantial effort. 
2. Acquisition cost, including capital.

Hardware: \$100K per system

Software: \$50K per system

3. Volume of input material, toxicity level, mobility, and physical state.

Not applicable. This is a support technology for characterization.

4. Volume of contaminated material produced.

Not applicable. This is a support technology for characterization.

5. Time to remediate input volume.

Not applicable. This is a support technology for characterization.

6. Time to remediate $50 \%$ of the input volume.

Not applicable. This is a support technology for characterization.

7. List the process output, their waste classification, concentration or activity, toxicity level, mobility, and volume.

Not applicable. This is a support technology for characterization.

8. Time until (a) line item and capital equipment replacement costs exceed $50 \%$ of acquisition cost and (b) line item and capital equipment and repair and maintenance costs are estimated to approach $50 \%$ of acquisition costs. Compare to expected useful life (see item a above).

Estimated that computers would be replaced (at $\$ 100 \mathrm{~K}$ each time) every 5 years per fielded system. Software upgrades estimated at $\$ 50 \mathrm{~K}$ per fielded system per 5 years.

9. Assemblies and subassemblies specified in the design and functional operating requirements and longest procurement lead times for custom and engineered components.

Not applicable to this technology. Uses standard computer equipment.

10. Total number of custom assemblies and subassemblies.

Not applicable to this technology. Uses standard computer equipment.

11. Time for scheduled maintenance.

One hour for each 100 hours of operation, based on workstation maintenance experience. 
12. Time for unscheduled maintenance.

Unknown but anticipated to be consistent with experience base for standard workstation equipment.

13. Time from now until field demonstrations. Now is considered to be the approval date of the technical evaluation report.

Work is expected to result in incrementally improving systems. Initial laboratory-scale demonstration is scheduled for 1995.

14. Time from now until full-scale operation.

Estimated to be ready in 1997.

15. Mean years of specialized training required for full-scale operation (academic and facility specific).

Assumed operation by a college-degreed geophysicist. Some support needed from a college-degreed engineer or computer scientist.

16. Labor hours per hour of operation.

Estimated as two people (full-time) per hour of operation. One person is assumed to be a geophysicist who is the digface characterization support expert.

17. Time for replacement parts to exceed $50 \%$ of the acquisition costs (see item 8).

Unknown, see item 8.

18. The list of permits required for full-scale operation.

Not applicable. This is a support technology for characterization.

19. Time to construct or fabricate equipment needed to operate the process.

Not applicable. Off-the-shelf equipment is assumed.

20. Types and quantities of utilities (e.g., water, electricity) required during the process.

Power for computer is required. Infrastructure to support other operations is assumed.

21. Time to train technical personnel and operators.

Expertise identified in item 15 is assumed. Approximately 40 hours of hardware-specific training is assumed. 
22. Extra time and costs prudently estimated to cover contingencies (i.e., unplanned events outside the control of the principal investigator and the BWID program.)

Unknown at present.

23. Discuss the status of commercializing this technology. List partners and mechanisms to be used to achieve goal.

This item is addressed in detail in Section 7.3 below.

\subsection{Related Efforts}

Research on high performance computer graphics, VEs, virtual reality, artificial reality, and simulation-based planning and design is currently being conducted at industrial, government, and academic sites in the United States and around the world. Virtual reality research and applications are being developed at several university and government research laboratories and in private industry. Some cooperative efforts between industry and universities are also in place. Several examples of efforts specifically directed at waste characterization are noted below.

- Mechanical Technology, Inc. Interactive Computer Enhanced Remote Viewing System

ICERVS is a computer-based system that provides data acquisition, data visualization, data analysis, and model synthesis to support robotic remediation in hazardous environments. The product is under development at Mechanical Technology, Inc. in Latham, New York (Mechanical Technology, Inc. 1993a). Robotic remediation is assumed due to the hazardous nature of the excavation operations when performed in buried waste. Use of robotic techniques is assumed to require building a three-dimensional model of the workspace, which are based on solid modeling methods where objects are represented by enclosing surfaces (e.g., polygons, quadric surfaces). The primary objective of ICERVS is to provide a reliable description of a workspace based on dimensional measurement data and to convert that description into three-dimensional models that can be used by robotic systems.

The workspace three-dimensional model is expected to be particularly applicable to situations such as tank remediation or decontamination and decommissioning where a structured description of the workspace exists. In environments such as a digface, which are unstructured and constantly changing, the effort to prepare a usable workspace model by ICERVS may not be worth doing. Nevertheless, there are aspects of the ICERVS system that can support the data display and data interpretation at the digface.

The VE project met with Mechanical Technology, Inc. scientists in February to view a structured light demonstration and discuss ICERVS capabilities. Based on this meeting, a copy of ICERVS was sent to be loaded on the project workstation at the INEL. Due to a number of unresolvable incompatibilities, the software did not work. We decided to wait until a newer upgraded version of ICERVS was available. In late 1994, a new version will be available for installation at the INEL. Based on testing and evaluation of both ICERVS and existing project software capabilities, features will be integrated where possible into a single 
system. Additionally, Mechanical Technology, Inc. is planning to extend ICERVS into a Phase 3 prototype system.

Mechanical Technology, Inc. and INEL VE scientists may collaborate on mutual areas of interest for advancing the methodologies of data interpretation and display of information from multiple, disparate sensors.

- Coleman Research Data Fusion Activities

Coleman Research Company of Maryland is developing; a geophysical data fusion technique to combine data from complementary sensors and incorporate geophysical understanding to obtain three-dimensional images of the subsurface. This data fusion technique uses information obtained from one sensor to constrain the mathematical inversion technique applied to the other sensor. Initial work used time domain EM data and seismic data (Hoekstra et al. 1993). Extension of the work is in progress.

\subsection{Commercialization}

Preliminary work evaluated the potential market for the VE terhnologies under development. ${ }^{m}$ The market assessment included an initial evaluation of factors that should be considered in system development from the point of view of ultimate product usefulness and commercialization potential.

The potential market for buried waste remediation technology is large and extends beyond Federal installations such as DOE and U.S. Department of Defense (DOD) sites. The Federal sites represent a significant potential investment of resources for cleanup; U.S. Environmental Protection Agency (EPA) estimates of costs range in the hundreds of billions of dollars. Although specific decisions have not been made regarding if and when most of these sites will be remediated, it appears safe to assume that technologies supporting buried waste cleanup will be commercially valuable, if they are technically useful. Preliminary market survey supports the project assumption of usefulness of VE technologies, based on feedback from businesses involved in the remediation marketplace. The commercial support is directed toward an encompassing suite of technologies including VE technologies and assumes a fairly sophisticated site characterization capability as part of the VE system. This support matches the general BWID development goal in developing characterization sensor and data interpretation. If successful, the commercial market will use and benefit from the technologies.

Besides buried waste remediation uses, a number of related fields were considered as potential markets for spin-off products from the buried waste VE work. These included the public utilities industry (e.g., underground gas and water lines), land-use planning and construction industries (imaging and displaying subterranean infrastructure), and industrial archeology (detecting underground structures). Similar to the buried waste characterization problem, all of these side markets are potential users of VE technology, assuming that the techniques are integrated with a sophisticated sensing and detection capability. It appears that the market support found in the initial survey is largely predicated on further development of both sensor capabilities and VE display capabilities.

m. R. Jacobson, personal communication (Worldesign Interim report), September 1994. 
The usefulness of tools and techniques under development will be influenced by anticipated increases in capabilities of virtual world technologies in general. (For example, as hardware becomes cheaper and faster, the system is accessible to more potential users.) An evaluation of general technology advancement and potential effects on the project tools was undertaken. It may be that by 1996, economical virtual worlds applications running on PC workstations will be the rule rather than the exception. Based on this possibility, the project is advised to plan for applications running on down-scalable, less-costly virtual world platforms. The general trend toward greater capability at less cost would be beneficial to this technology, except if newer off-the-shelf capabilities are also associated with a movement away from Unix operating systems. In that case, substantial upgrade/rework may be needed to keep in tune with marketplace tools.

From a commercialization standpoint, the current state-of-the-art immersion technologies, such as military HMDs, may be too large and expensive to be included in products for commercial use. Projection technologies such as CAVEs and Virtual Environment Theaters ${ }^{\text {Th }}$ (VETs ${ }^{\text {Th}}$ ) may be too large, expensive, and immobile for use in this application. At present, the creation of virtual worlds to be visualized on two-dimensional video monitors may make the most sense.

The lack of standardization in the arena of virtual worlds software is a general problem in this field and will affect commercial potential of project products. However, it is anticipated that by 1996, the market for virtual worlds software will change remarkably. Constant scrutiny of this area and adjustment of project tools and software has been advised. 


\section{CONCLUSIONS, RECOMMENDATIONS, DEVELOPMENT NEEDS}

- Rapid Prototype Study (conclusions drawn about development system requirements)

- The environment must accommodate and use large layered data sets that will be encountered in digface characterization/remediation type operations.

- The environment must accommodate multivariate data because of the variety of sensors that may be involved in digface operations.

- The environment should have the ability to handle data gathered on or representative of a wide variety of physical coordinate spaces.

- The environment should be capable of displaying information in a wide variety of ways due to the diverse range of potential users.

- The environment should provide the supporting structure in which domain experts may conceive, implement, and test and refine new empirical algorithms for description, identification, and classification as warranted based on their perception of the data.

- Integrated Hardware and Software Platform

- The architecture for an integrated software and hardware platform must integrate a number of capabilities and include hooks to accommodate future capabilities. Architecture of the system under development is shown in Figure 4-1.

- This system must have ready access to previously recorded data as well as to data that are being gathered and should allow the analyst/gecphysicist to work with these data sets in whatever manner desired. The overall analysis/visualization approach taken is to integrate multiplatform data analysis software and an advanced RDMS with VE generation software. Such integration will ideally allow the different programs involved to respond to calls from each other allowing automation of data storage, analysis, and display.

\section{Recommendations}

- The prototype system should be formally reviewed by potential users, specifically including Environmental Restoration personnel. Additional development should be guided by reviewer comments.

- Depth-to-Object Algorithms

- The following analysis areas were highlighted as being of major interest:

- Depth-to-object estimation

- Contour analysis (determination of anomaly extrema) 
- Software to perform depth-to-object analyses has been implemented and verified. The algorithm provides reasonable estimates of the object depth, although with a wide range of estimates. Depths estimated using the digface and RGS data were typically within one-half foot of real values.

\section{Recommendations}

- The automation of the depth estimation algorithms allows verification to be done on numerous data. Such checking is too tedious and time consuming to be done by hand. The algorithms should be checked on numerous data sets, comprising a range of targets and environments. The 1994 digface data set is one specific data set on which the algorithms should be checked.

- Methods of estimating uncertainty in the calculated results should be investigated further. The capability for automated analysis allows a more systematic approach to uncertainty. Estimation of uncertainty supports a more rigorous approach to establishing confidence in the results for use in decisionmaking during hazardous waste remediation.

- Much work remains to be done to advance the state-of-the-art in near-field geophysics data interpretation and integration of data from various sensor types. Detailed analysis of the existing high resolution data sets to determine appropriate interpretation and algorithms to support this interpretation is warranted. Contour analysis and anomaly characterization of magnetic and EM sensor data are topic areas of particular benefit.

\section{- ATR System Evaluation}

- The ATR system performance was generally good for the data interpretation application. (For this initial study, the evaluation was done under rather ideal conditions.) Although there was some errors in distinguishing boxes from barrels, the system is capable (based on the limited testing done) of distinguishing target objects from background soil.

- Results show that application of the target recognition system was consistent with manual human data interpretation in that the definition of targets clearly improves as the sensors are brought closer to the waste.

\section{Recommendations}

- Initial results indicate that further evaluation and possible development of the ATR system are warranted. Initial results are encouraging; however the ATR system must be demonstrated under conditions more representative of typical buried waste sites. The reasonable success when searching for defined and resolvable targets needs to be demonstrated in complex environments representative of real waste sites.

- In future assessment, the ATR system training should also be directed toward distinguishing target from background only, in addition to classifying specific types of 
targets. This capability (distinguishing target or background) is currently of interest as is classifying target types.

- The limitations of the system in handling missing data (such as a sensor missing from the surveillance data that was present in the training data set) should be evaluated. Under such conditions, if the system performs poorly without reasonable chance for improvement through development, the likelihood of value in the digface application is questionable.

- Evaluation should be made of the potential for the system to use volumetric data (information obtained from the same sensor at different levels, i.e., different distances from the targets search area). Based on likely digface application, information from two (or possibly three) levels should be considered. The evaluation should include assessing performance degradation when same level parameters varies in surveillance data from what was used in the training set. It should be noted that inability to use multiple level data would not necessarily eliminate the ATR as a useful tool for the digface application.

\section{- Neural Net Evaluation}

- The neural network performance was generally good for the data interpretation application. After undergoing a training process, the networks demonstrated reasonable capability to distinguish targets from background. The networks have been able to recognize target locations accurately on over $85 \%$ of the digface data.

- Both backpropagation and RBF neural network paradigms were used for target background classification. For backpropagation networks using one data level and one sensor type, the number of input PEs was the size of the input vector (e.g., two for magnetics, four for EM) and the size of the hidden layer was generally one less than the number of input PEs. One output PE was used. Training times for 20,000 iterations were typically 30 seconds. Typical testing times vrere of the order of 3 seconds for one sensor and one level of digface data. RBF networks were used when backpropagation did not produce adequate results. The input vector was generally the same as that for the backpropagation networks. One hidden layer was used along with an extra layer for the Gaussian kernel functions. One output PE was used. Typical training times were 2 minutes for 50,000 iterations and typical testing times were 5 seconds for one sensor and one level of digface data.

- Training networks using combined sensor types within a data level provided some improvement in classification capability, particularly when objects with strong magnetic signatures were present in the experiment. Typically, RBF networks with six input, three hidden PEs, and one output PE were used. Training times for 50,000 iterations were from 2 to 4 minutes. Testing time for a single layer of data was approximately 6 seconds.

- Using multiple levels of data and combining sensor types for training and testing modes resulted in a better definition of target boundaries. Backpropagation networks with two hidden layers gave best results. Typically, the first hidden layer had one less PE than 
the input vector (input vectors ranged from 12 to 44 depending on the experiment), and the second hidden layer had half as many PEs as the first. Training times for 70,000 iterations averaged 5 minutes and testing times were of the order of 7 seconds.

- Data from several experiments were combined to find a training set that would produce good results on all experiments. Targets from each experiment (except Experiments 1 and 4) had to be included to get good results. Both magnetic and EM data were included and level data were accumulated. Training times were significant, but testing times were less than 10 seconds.

- Preliminary indications support the potential ability of neural networks to provide reasonable depth estimates. All results to date are within about $10 \%$ error of the desired depths. Additional evaluation is in progress.

- Neural network performance on test sets including magnetic components was less affected by disabling certain EM survey components. Up to two survey components could be disabled without significant degradation of the net performance on these test sets.

- In general, the EM survey finds more targets more accurately than does the magnetic survey. However, if any EM component is missing the net performance can be degraded. When EM and magnetic survey data are combined, then net performance is not damaged appreciably when one or even two EM or magnetic components are unavailable. In other words, at least four input vectors seem to be required and it does not seem to matter (at least at this stage of the analysis) which four vectors.

\section{Recommendations}

- Initial results indicate that further evaluation and development of neural networks are warranted. Initial results are encouraging; however, the networks must be demonstrated under conditions more representative of actual buried waste sites. The reasonable success when searching for defined and resolvable targets needs to be demonstrated in environments representative of real waste sites.

- Network performance should be evaluated under conditions in which missing sensor data are encountered. A systematic study is needed to define expected performance under a range of reasonable missing data scenarios.

- Network performance should be evaluated under conditions in which the spatial grid for surveillance data are substantially degraded from the training sets.

- An evaluation of preprocessing methods such as wavelet preprocessing should be undertaken. The objective should be an understanding of the potential enhancement in network performance, including training and run times, that might result from preprocessing. 
- The visual data base applications showed the ability, benefit, and utility of having different sources of information about a particular waste site gathered into a centralized location. The applications investigated included the CTP Characterization Cell and Pit 9. For the Characterization Cell application data from the digface Characterization activities were used in the development. For the Pit 9 application, historical data from the existing RWMIS data base and additional information generated during CIDRA activities was used. While analysis capabilities discussed in Section 5.3 have yet to be fully implemented into this environment, the potential benefit of such abilities is clear.

- Although the Characterization Cell application was not particularly large (total of approximately 20 objects), the need to have computing capabilities with significant power was demonstrated. The visual data base implementation was run on several different classes of work stations. On lower end (older) machines that did not contain dedicated graphics processors, the response to user movement through the objects was irritatingly slow as were screen updates and responses to queries. On newer machines (e.g., Sun Sparc10 with dedicated graphics processors), the performance was much better and movement through the objects in the world was quite acceptable.

- Based on implementation experience with the Pit 9 data base, it is clear that there will always be some doubts about the accuracy of the information in such a historical data base. The information must be used with caution and human expertise, and experience must be used in the process of building and using a visual data base.

\section{Recommendations}

- Additional analysis capabilities, as suggested in Section 3.5 should be incorporated into the system.

- The system should be formally reviewed by potential users, specifically including Environmental Restoration personnel. Subsequent development, if warranted, should be guided by reviewer comments.

\section{- Immersion Evaluation}

- A limited evaluation of immersion capabilities indicates that polygon rendering is more important than texture mapping at this time. Improved polygon rendering and pixel transfer rates are needed to maintain user comfort as scene complexity increases.

- Current near-field geophysics data interpretation requires substantial training and expertise and is in an immature state. Until techniques are developed that would support representation of data by physical world objects, it is not clear how immersion will benefit geophysical data interpretation. 
Immersion will play a larger role in the planned use of stereo/video and stereo/video mixing with VEs. Immersion will improve depth perception of people operating telerobotic equipment in hazardous environments. Further evaluation is planned in 1995.

- Stereo glasses coupled with appropriate monitors are adequate for tasks not requiring the sense of immersion. For training, potential direct operator control and other tasks, full immersion is necessitated by the need for the user to feel immersed in the virtual world.

\section{Recommendations}

- The use of stereovision to improve depth perception should be investigated and performance evaluated in 1995. Reasonable frame rates must be provided to allow users to benefit from the sense of immersion.

- The use of immersion to support geophysical data interpretation should be placed at lower priority until a better fundamental understanding exists in the field of near field geophysics. At present, insufficient fundamental understanding in the field exists to allow evaluation of immersion techniques for potential benefit.

\section{- Commercialization}

- The potential market for buried waste remediation technology is large and extends beyond Federal installations such as DOE and DOD sites. The Federal sites themselves represent a significant potential investment of resources for cleanup; EPA estimates of costs range in the hundreds of billions of dollars.

- Preliminary market survey supports the project assumption of usefulness of VE technologies, based on feedback from businesses involved in the remediation marketplace. The commercial support is directed toward an encompassing suite of technologies including VE technologies and assumes a fairly sophisticated site characterization capability as part of the VE system.

- Several potential markets for spinoff products from the buried waste VE work have been identified. These include the public utilities industry (underground gas and water lines), land-use planning and construction industries (imaging and displaying subterranean infrastructure), and industrial archeology (detection of underground structures). Similar to the buried waste characterization problem, all of these side markets are potential users of VE technology, assuming that the techniques are integrated with a sophisticated sensing and detection capability. It appears that the market support found in the initial survey is largely predicated on further development of both sensor capabilities and VE display capabilities.

- From a commercialization standpoint, the current state-of-the-art immersion technologies, such as military HMDs, may be too large and expensive to be included in products for commercial use. Projection technologies such as CAVEs and VETs ${ }^{\mathrm{mm}}$ may be too large, expensive, and immobile for use in this application. At present, the 
creation of virtual worlds to be visualized on two-dimensional video monitors may make the most sense.

- The lack of standardization in the arena of virtual worlds software is a general problem in this field and will affect commercial potential of project products. However, it is anticipated that by 1996, the market for virtual worlds software will change remarkably. Constant scrutiny of this area and adjustment of project tools and software is advised.

\section{- Additional Recommendations}

A detailed review of existing data and measurement methods should be undertaken to evaluate current near field geophysical sensing capabilities and potential role in supporting an integrated strategy for buried waste characterization. Advancements made in 1994 by BWID funded technologies (such as Very Early Time Electromagnetics and holographic GPR) should be specifically looked at in the context of prioritizing information needed to support the overall characterization effort. The usefulness of data from specific sensors should be examined in the context of how quickly and how well the information can be transmitted to people needing it. The role of the VE project techniques in enhancing the usefulness of the data is one factor among others that should be considered. Another factor to consider is the amount of information collected by a specific sensor is completely independent of data collected by other sensors. The VE-integrated platform may provide a tool to assist in evaluating the usefulness of various sensor data by being a central focus of data collection, storage, and display.

The existing high resolution data sets (1993 and 1994 digface data) are rich in content. These data should be further exploited by a detailed engineering analysis to determine the range of information present in the data. The data have not yet been investigated in a systematic way to explore different ways of understanding, displaying, or analyzing the data to determine how much information can be brought forth from the data and exploited to improve understanding. A close and careful review and analysis of the data will support advancement of techniques for fundamental understanding of near field geophysics. 


\section{REFERENCES}

Anderson, J. and E. Rosenfeld, 1989, Neurocomputing: Foundations of Research, Massachusetts Institute of Technology Press.

Advanced Visual Systems (AVS), 1993, Application Visualization System (AVS), Advanced Visual Systems, Inc., Waltham, Massachusetts.

Baum, E. and D. Haussler, 1989, "What Size Net Gives Valid Generalization?" D. Touretzky (ed.), Advances in Neural Information Processing Systems I, Morgan Kaufmann Publishers, pp 81-91.

Bonnenberg, R. W., 1994, Buried Waste Integrated Demonstration Program Technology Report Guidance, EGG-WTD-10104, Revision 2, January.

Caudill M., 1988, "Neural Networks Primer, Part IV," AI Expert: August, pp. 61-67.

Clements, T. L. Jr., 1982, Content Code Assessments for INEL Contact-Handled Stored Transuranic Wastes, WM-F1-82-021, EG\&G Idaho, Inc., October.

Coleman, 1993, INEL Cold Test Pit Demonstration of Improvements in Information Derived from Non-Intrusive Geophysical Methods Over Buried Waste Sites, PHASE I-Final Report, PRDA No. DE-RA05-910R22000, Coleman Energy \& Environmental Systems-Blackhawk Geosciences Division (Coleman Research Corporation), September.

Dayoff, J., 1989, Neural Network Architectures, Van Nostrand Reinhold.

Freeman, J. and D. Skapura, 1991, Neural Networks: Algorithms, Applications, and Programming Techniques: Addison-Wesley, New York.

Hoekstra, P. et al., 1993, Geophysical Data Fusion for Subsurface Imaging, Phase 1 Topical Report, Coleman Research Corporation, August.

Josten, N. E., 1992, Preliminary Design for a Safe-Step Remediation System, EG\&G Idaho, Inc., EGG-EELS-002, September.

Josten, N. E. and L. G. Roybal, 1993, Rapid Geophysical Surveyır Final Report, EGG-WTD-10566, EG\&G Idaho, August.

Josten, N. E. and L. G. Roybal, 1994, Test Plan for FY-94 Digface Characterization Field Experiments, EGG-WTD-11424, EG\&G Idaho, Inc., August.

Josten, N. E., 1995, Progress on Development of the Digface Characterization Technology, INEL95/0093, Lockheed Idaho Technologies Company.

Kohonen, T., 1988, Self-Organization and Associative Memory, Springer-Verlag New York, Inc. 
Lassahn, G. D., 1993, Preliminary Evaluation of an ATR System Applied to Robotic Tank Inspection, EGG-EE-11040, EG\&G Idaho, Inc., October.

Leonard, J. and M. Kramer, 1991, Radial Basis Functions for Classifying Process Faults: IEEE Control Systems, April.

Litteer, D. L., 1988, Idaho National Engineering Laboratory Radioactive Waste Management Information System (RWMIS) Solid Data Users Manual, DOE/ID-10188 Revision 2, EG\&G Idaho, Inc., September.

Meachum, T. R., 1993, Areas and Volumes for Selected Radioactive Waste Management Complex Pits and Trenches, ER\&WM-EDF-0007-93, EG\&G Idaho, Inc., June.

Mechanical Technology Inc., 1993a, ICERVS System Design Report, Phase 2, Mechanical Technology Incorporated, Latham, New York, November.

Mechanical Technology, Inc., 1993b, Interactive Computer-Enhanced Remote Viewing System (ICERVS), Volume II: Phase II Proposal, Mechanical Technology, Inc., MTI Q3-419, May.

Mehrota, K., C. Mohan, S. Ranke, "Bounds on Number of Samples Needed for Neural Learning," IEEE Trans. on Neural Networks, 2, pp 548-558.

Moody, J. and C. Darken, 1989, "Fast Learning in Networks of Locally-Tuned Processing Units," Neural Computation 1, pp. 281-294.

Musavi, M. et al., 1992, "On the Training of Radial Basis Function Classifiers," Neural Networks 5, pp. 595-603.

NCSA Mosaic, 1994, NCSA Mosaic 1.0.3, Copyright 1994, National Center for Supercomputing Applications, University of Illinois, Urbana-Champaign, Software Development Group, Champaign, Illinois, January 1994.

Parker, D., 1989, Learning Logic: Invention Report S81-64 File 1, Office of Technology Licensing, Stanford University, Stanford, California.

Partin, J. K., G. D. Lassahn, J. R. Davidson, 1994, Automatic TLI Recognition System, Part 1: System Description, EGG-11298, EG\&E Idaho, Inc., May.

Poggio, T. and F. Girosi, 1990, "Regularization Algorithms for Learning that are Equivalent to Multilayer Networks" Science, 247, pp. 978-982.

Poulton, M. M. et al., 1992, "Neural Network Pattern Recognition of Subsurface EM Images," Journal of Applied Geophysics, 29, pp 21-26.

Poulton, M. M. and M. P. Brown, 1994, Feasibility Study on the Applicability of Artificial Neural Networks for the Interpretation of Digface Data, Laboratory of Advanced Subsurface Imaging, University of Arizona, Lockheed Idaho Technologies Company, December. 
Press, W. H. et. al., 1987, Numerical Recipes-The Art of Scientific: Computing, Cambridge University Press.

Rod, S. R., 1992, Graphics-Based Waste Site Management, Presentation at Air \& Waste Management Association 85th Annual Meeting \& Exhibition, Kansas City, Missouri, June 21-26.

Rumelhart, D., G. Hinton, J. McClelland, 1986, A General Framework for Parallel Distributed Processing, in D. Rumelhart, and J. McClelland, (Eds.), Parallel Distributed Processing, Vol 1: Massachusetts Press, pp. 45-76.

Sandness, G. et al., 1993, Remote Characterization System Technology Evaluation Report, EGG-2691, EG\&G Idaho, Inc., September.

Sense8 Corporation, 1994, WorldToolKit, Users Manual, Sense8 Corporation, Sausilito, California.

Smith, T. H. et al., 1994, A Comprehensive Inventory of Radiological and Nonradiological Contaminants in Waste Buried in the Subsurface Disposal Area of the INEL RWMC During the Years 1952-1983, EGG-WM-10903, EG\&G Idaho, Inc., June.

Soulie, F. et al., 1987, Evaluation of Neural Network Architectures on Test Learning Tasks, 1st Internat. Conf. on Neural Networks, IEEE Proc., pp 653-661).

Vacquier, V. et al., 1951, "Interpretation of Aeromagnetic Maps," Geologic Society of America Memoir 47, New York.

VIRTUS Corporation, 1993, VIRTUS Walkthrough Pro, Version 1, User's Manual, VIRTUS Corporation.

VREAM, Inc., 1993, VREAM Virtual Reality Development System, Reference Manual 1.0, VREAM, Inc., Chicago, Illinois.

Wasserman, P., 1989, Neuralcomputing: Theory and Practice, Vin Nostrand Reinhold.

Werbos, P., 1974, Beyond Regression: New Tools for Prediction and Analysis in the Behavioral Sciences, Ph.D. Dissertation, Harvard University. 
Appendix A

FY-93 Digface Performance Tests

N. E. Josten 
A-2 


\section{Appendix A}

\section{FY-93 Digface Performance Tests}

\section{A-1. SUMMARY}

The digface performance tests were conducted at the Idaho National Engineering Laboratory's (INEL's) Cold Test Pit (CTP). Three experiments were conducted at the Characterization Cell of the CTP. The Characterization Cell contains a number of simple isolated objects comprised of materials representing typical U.S. Department of Energy (DOE) low-level waste. The remainder of the digface performance tests were conducted at the Experiment Cell, which was specially built for the digface project. The Experiment Cell was created to provide a variety of simple examples of magnetic and electromagnetic (EM) signal superposition and interaction from multiple bodies. The trolley was used to scan the set of sensors over six test sites. The test sites were then excavated in incremental layers ranging from 0.5 to $1 \mathrm{ft}$ thick. After each excavation, new scans were performed at the new surface elevation. Data were acquired and stored by the data acquisition system. The process continued until the objects buried in each cell were exposed. Over 200,000 data points were collected during a 6-week period from late September to the end of October 1993. Although data acquisition operations were manually driven, the data were collected efficiently and of high quality. The gantry crane deployment approach provided a stable and precise platform for data acquisition.

\section{A-2. PERFORMANCE TEST FESULTS}

The primary objective of the digface performance tests was to demonstrate collecting characterization data at the digface so that remediation efforts can be directed as needed in the field based on real-time characterization. To illustrate this concept, a scenario of ideal conditions for data collection was constructed to minimize signal interference from external objects including the structure supporting the sensor delivery and data collection systems.

\section{A-2.1 Test Description}

The digface performance tests were conducted at the INEL's CTP. The CTP contains a series of simulated waste areas designed to imitate configurations found in radioactive/hazardous burials areas throughout the DOE complex. The CTP is located a few hundred yards south of the Subsurface Disposal Area (SDA) within the Radioactive Waste Management Complex. The SDA is the main low-level radioactive waste burial ground for the INEL.

The original CTP $^{a}$ was constructed in 1988 and contained five distinct burial cells. The original construction was $145 \mathrm{ft}$ long $\times 40 \mathrm{ft}$ wide and oriented so that the long axis was approximately aligned south to north. In November 1992 , two additional test cells ${ }^{\mathrm{b}}$ were added approximately $40 \mathrm{ft}$ north of the existing CTP. These cells were designed and constructed to support a number of Buried Waste Integrated Demonstration Program tests performed during the summer of 1993 and were designated the

a. G. G. Loomis, "Design and Construction Details of the INEL Simulated TRU Test Pit," EG\&G Idaho, Inc., Engineering Design File BWP-150-009, March 2, 1989.

b. M. R. Winberg, "Specifications of the Addition of a Characterization and a Retrieval Cell to the Cold Test Pit (As-Built), “ EG\&G Idaho, Inc., Engineering Design File, December 29, 1992. 
Characterization Cell and Retrieval Cell, respectively. The Retrieval Cell was later excavated and removed in August 1993.

Three of the six digface performance tests were conducted at the Characterization Cell during September and October 1993. A schematic of the Characterization Cell is shown in Figure A-1. The Characterization Cell contains a number of simple isolated objects comprised of materials representative of typical DOE low-level waste. This includes boxes filled with metallic and nonmetallic debris and 55-gal drums. Also, two metal filing cabinets representing large heavy debris are located in the extreme eastern part of the Characterization Cell. Table A-1 provides a detailed listing of the contents of the Characterization Cell.

The remainder of the digface performance tests were conducted at the digface Experiment Cell, which was built for the digface project. The Experiment Cell was created to provide a variety of simple examples of magnetic and electromagnetic signal superposition and interaction from multiple bodies. A schematic of the Experiment Cell is shown in Figure A-1. The pit contains a variety of objects in multiple configurations including empty and full wooden boxes, 5-gal metal pails as single objects and stacked geometries, reinforced concrete, a large automobile engine, and a number of long linear objects (e.g., pipes, I-beams) made from ferrous and nonferrous metals and plastics. A list of buried objects in the Experiment Cell is provided in Table A-2.

The performance tests were conducted using a custom built manual three axis trolley. The trolley was set up over each test location and used to scan a set of noninvasive sensors over the test cell. The cells were then excavated in incremental layers ranging from 0.5 to $1 \mathrm{ft}$ thick. After each excavation, new scans were performed at the new surface elevation. Data were acquired and stored by a PC-based data acquisition system. The process continued until the objects buried in the cell were exposed.

\section{A-2.1.1 Sequence of Activities}

A detailed description of the experimental site activities is provided in an informal EG\&G Idaho test plan (Josten and Roybal 1993). The general scope of activity was to collect geophysical and chemical data in multiple parallel planes that moved progressively closer to buried target objects as soil overburden was removed. The trolley survey footprint was much smaller than the Characterization and Experiment Cells so that each survey area required three overlapping trolley surveys. Six trolley positions were required to provide complete coverage of the two cells. Therefore, the initial activity before data collection involved positioning (including absolute positioning with respect to a global coordinate system), squaring, and leveling the trolley. Once this was accomplished, the pad surveys proceeded in a series of elevation steps, beginning with a survey of the air space above undisturbed ground and continuing belowgrade with soil layers removed in 6 in. to $1 \mathrm{ft}$ increments until waste was encountered (see Figure A-2). Generally, spatial increments of 3 in. in the $x$-direction, 6 in. in the $y$-direction, and 6 in. in the $z$-direction were used for all surveys. Special increments along the $z$-axis were used for the chemical sensor to maintain proximity to ground level.

While the general procedures and activities for each experiment were similar, each experiment had unique attributes. The most obvious were the varying target types in each experiment. Since the targets were known, the experiments had different goals and emphasis. A short synopsis of each experimental setup used during the performance tests is provided below. 


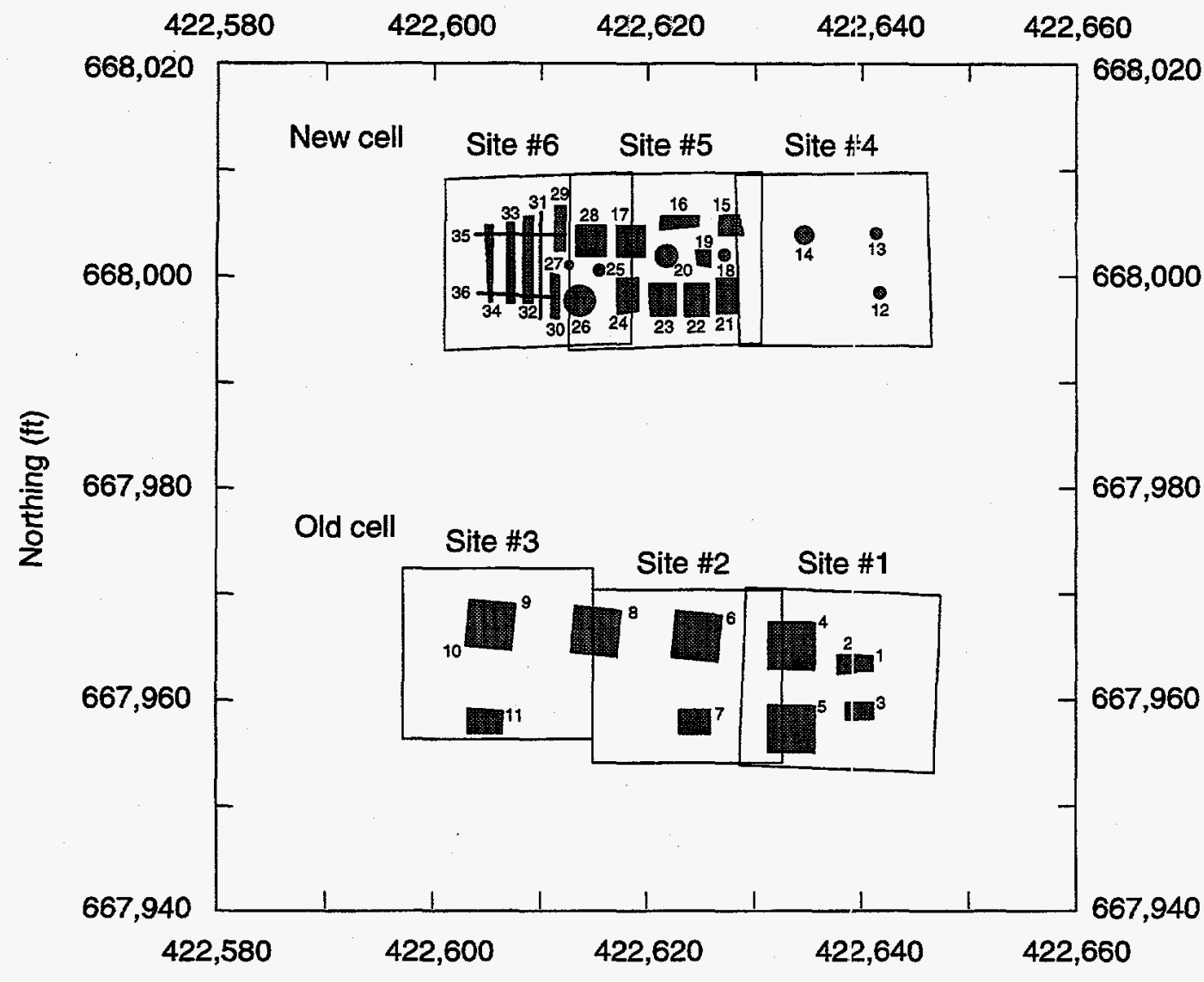

Easting (ft)

\begin{tabular}{|c|c|c|c|c|c|}
\hline \multirow[b]{2}{*}{$\begin{array}{l}\text { Object } \\
\text { ID }\end{array}$} & \multicolumn{2}{|c|}{ Old cell } & \multirow[b]{2}{*}{$\begin{array}{l}\text { Object } \\
\text { ID }\end{array}$} & \multicolumn{2}{|c|}{ New cell } \\
\hline & Description & Notes & & [lescription & \\
\hline 1 & File cabinet & & 12 & Alcohol plume & 5-gal point source \\
\hline 2 & $\begin{array}{l}\text { File cabinet } \\
\text { cover }\end{array}$ & Loose from 1 & $\begin{array}{l}13 \\
14\end{array}$ & $\begin{array}{l}\text { Alcohol plume } \\
\mathrm{NaCl} \text { plume }\end{array}$ & $\begin{array}{l}\text { 5-gal point source } \\
5 \text {-gal }\end{array}$ \\
\hline 3 & File cabinet & & 15 & 5-gjal pails & Vertical stack, 4 on 4 \\
\hline 4 & Wooden box & Contains metal scrap & 16 & 5-gjal pails & Pyramid stack, 1-2-3 \\
\hline 5 & Wooden box & Contains wood and paper & 17 & 55.gal barrel & Horizontal, empty \\
\hline 6 & Wooden box & Contains nonferrous metal & 18 & 5-gjal pail & Vertical, empty \\
\hline 7 & 55-gal barrel & Contains foam & 19 & 5-cjal pail & Horizontal, empty \\
\hline 8 & Wooden box & Contains mixed metals & 20 & 55-gal barrel & Vertical, empty \\
\hline 9 & Wooden box & Contains wood and paper & 21 & Wooden box & Empty \\
\hline 10 & Wooden box & Beneath 9: contains ferrous & 22 & Wooden box & Contains dry dirt \\
\hline & & metal & 23 & Wooden box & Contains wet dirt \\
\hline 11 & 55-gal barrel & Contains concrete & 24 & Wooden box & Empty \\
\hline & & & 25 & Iron pipe & Vertical \\
\hline & & & 26 & Concrete block & 6-in. steel loop on top \\
\hline & & . & 27 & Stieel pipe & Vertical \\
\hline & & & 28 & Trusck engine & \\
\hline & & & 29 & PV'C pipe & Horizontal \\
\hline & & & 30 & Strel pipe & Horizontal \\
\hline & & & 31 & Copper bar & \\
\hline & & & 32 & Iren " $C$ " channel & \\
\hline & & & 33 & Allıminum bar & \\
\hline & & & 34 & Steel bar & \\
\hline & & & 35 & Alicohol plume & 15-gal poured in trench \\
\hline & & & 36 & $\mathrm{NaCl}$ plume & 15-gal poured in trench \\
\hline
\end{tabular}

RED GJOO15

Figure A-1. Layout of digface Experiment Cells (figure courtesy of N. E. Josten, INEL). 
Table A-1. Description of objects in Characterization Cell, used for Experiments 1, 2, and 3 of the FY-93 digface performance tests.

Object

identification

Description

Notes

\begin{tabular}{lll}
\hline 1 & File cabinet & - \\
2 & File cabinet cover & Loose from 1 \\
3 & File cabinet & - \\
4 & $4 \times 4 \times 2$ wooden box & Contains metal scrap \\
5 & $4 \times 4 \times 2$ wooden box & Contains wood and paper \\
6 & $4 \times 4 \times 2$ wooden box & Contains nonferrous metal \\
7 & 55 -gal barrel & Contains foam \\
8 & $4 \times 4 \times 2$ wooden box & Contains mixed metals \\
9 & $4 \times 4 \times 2$ wooden box & Contains wood and paper \\
10 & $4 \times 4 \times 2$ wooden box & Beneath 9; contains ferrous metal \\
11 & 55 -gal barrel & Contains concrete \\
\hline
\end{tabular}


Table A-2. Description of objects in digface Experiment Cell, used for Experiments 4, 5, and 6 of the FY-93 digface performance tests.

\begin{tabular}{|c|c|c|}
\hline $\begin{array}{c}\text { Object } \\
\text { identification }\end{array}$ & Description & Notes \\
\hline 12 & Alcohol plume & 5-gal point source \\
\hline 13 & Alcohol plume & 5-gal point source \\
\hline 14 & $\mathrm{NaCl}$ plume & 5-gal point source \\
\hline 15 & 5-gal pails & Vertical stack, 4 on 4 \\
\hline 16 & 5-gal pails & Pyramid stack, 1-2-3 \\
\hline 17 & 5-gal barrel & Horizontal, empty \\
\hline 18 & 5-gal pails & Vertical, empty \\
\hline 19 & 5-gal pails & Horizontal, empty \\
\hline 20 & 5-gal barrel & Vertical, empty \\
\hline 21 & $2 \times 4 \times 2$ wooden box & Empty \\
\hline 22 & $2 \times 4 \times 2$ wooden box & Contains dry dirt \\
\hline 23 & $2 \times 4 \times 2$ wooden box & Contains wet dirt \\
\hline 24 & $2 \times 4 \times 2$ wooden box & Empty \\
\hline 25 & Iron pipe & Vertical \\
\hline 26 & Concrete block & 6-in. steel loop on top \\
\hline 27 & Steel pipe & Vertical \\
\hline 28 & Truck engine & V8 \\
\hline 29 & PVC pipe & Horizontal \\
\hline 30 & Steel pipe & Horizontal \\
\hline 31 & Copper bar & - \\
\hline 32 & Iron " $\mathrm{C}$ " channel & - \\
\hline 33 & Aluminum bar & - \\
\hline 34 & Steel bar & - \\
\hline 35 & Alcohol plume & $15 \mathrm{gal}$, poured in furrow \\
\hline 36 & $\mathrm{NaCl}$ plume & $15 \mathrm{gal}$, poured in furrow \\
\hline
\end{tabular}



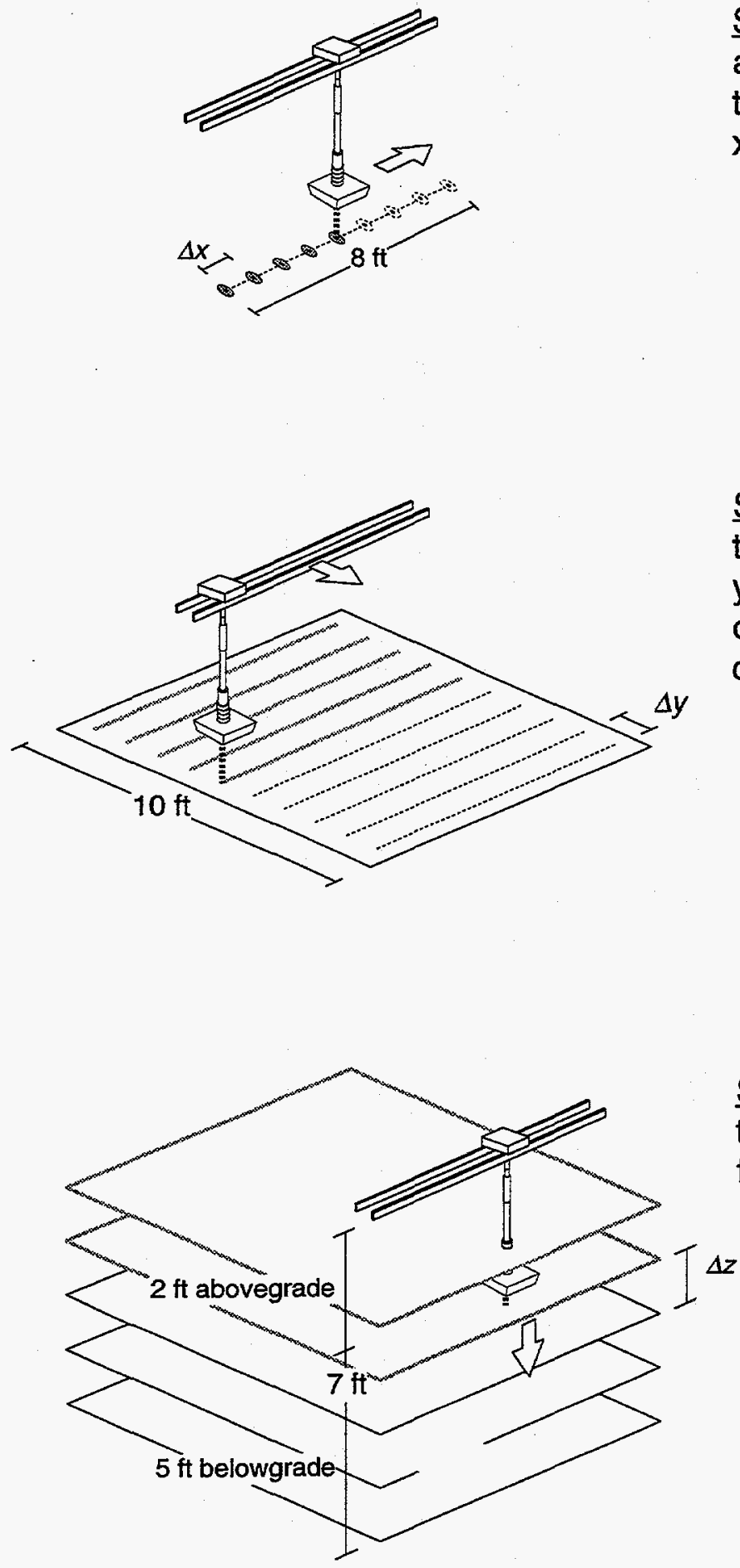

Surveying a profile automatic data collection as trolley manually scanned in the $x$ direction

\section{Surveying a level} trolley moved incrementally in the $y$ direction, followed by scanning of new $x$ profile until level survey complete
Surveying a cell

trolley mast lowered incrementally followed by new level survey

Figure A-2. Illustration of the trolley scan sequence for surveying the three dimensional volume above a test cell. Note the definition of the survey parameters $\Delta x, \Delta y$, and $\Delta \mathbf{z}$. 


\section{Experiment 1}

Experiment 1 was performed over the far eastern edge of the Characterization Cell as shown in Figure A-1. (Figure A-3 contains a detailed drawing of Experiment 1.) The dominating waste forms in this experiment were two large filing cabinets buried approximately $6 \mathrm{in}$. below the surface of the cell. The experiment area also covers about half of two large boxes buried about $3 \mathrm{ft}$ belowgrade. The northernmost box contains metal scrap while the southernmost box contains wood and paper products.

Four pad levels were scanned with a magnetic sensor, an EMI sensor in quadrature mode, and the EM sensor in inphase mode. The EM measurements were made with the sensor in a horizontal dipole configuration. This corresponded to 12 distinct pad surveys extending from the uppermost trolley position to about 3 in. belowgrade.

\section{Experiment 2}

Experiment 2 was performed over the middle portion of the Characterization Cell as shown in Figure A-1. (Figure A-4 contains a detailed drawing of Experiment 2.) Waste forms in this experimental setup included a large wooden box filled with nonferrous metals and a 55-gal drum filled with foam.

Nine pad levels were scanned with magnetic and EM sensors. The EM device was run in quadrature and inphase modes in both horizontal and vertical dipole configurations. Therefore, five sensors times nine pad levels were scanned for a total of 45 clistinct surveys extending from the uppermost trolley position to $4 \mathrm{ft}$ belowgrade.

\section{Experiment 3}

Experiment 3 was performed over the westernmost portion of the Characterization Cell as shown in Figure A-1. (Figure A-5 contains a detailed drawing of Experiment 3.) This experiment included double-stacked boxes located in the northern part of the Characterization Cell where the upper box contains wood and paper, the lower box contained ferrous metals, and a 55-gal drum, located in the southern portion of the pit, contained concrete.

Eight pad levels were scanned with magnetic and EM serisors. The EM device was run in quadrature and inphase modes in both horizontal and vertical dipole configurations. Therefore, five sensors times eight pad levels were scanned for a total of 40 distinct surveys extending from the uppermost trolley position to $3 \mathrm{ft}$ belowgrade. The upper box was removed from the survey area when the excavation reached about $0.5 \mathrm{ft}$ belowgrade.

\section{Experiment 4}

Experiment 4 was conducted over the westernmost portion of the digface Experiment Cell. This area was free from buried waste and designated specifically for contaminant plume detection. One salt and two alcohol plumes configured as shown in Figure A-2 were cleated prior to experimental surveys. (Figure A-6 contains a detailed drawing of Experiment 4.)

Four levels of horizontal dipole quadrature data, three levels of vertical dipole quadrature data, and three levels of volatile organic chemical (VOC) data were collected for a total of 10 distinct surveys. The quadrature data were collected starting from the uppermost trolley position downward. The VOC data were collected at levels 3, 4, and 5. This represents one pad level at original grade and two successive levels belowgrade after excavation. The abovegrade upper levels were not collected with the 


\section{Experiment 1}

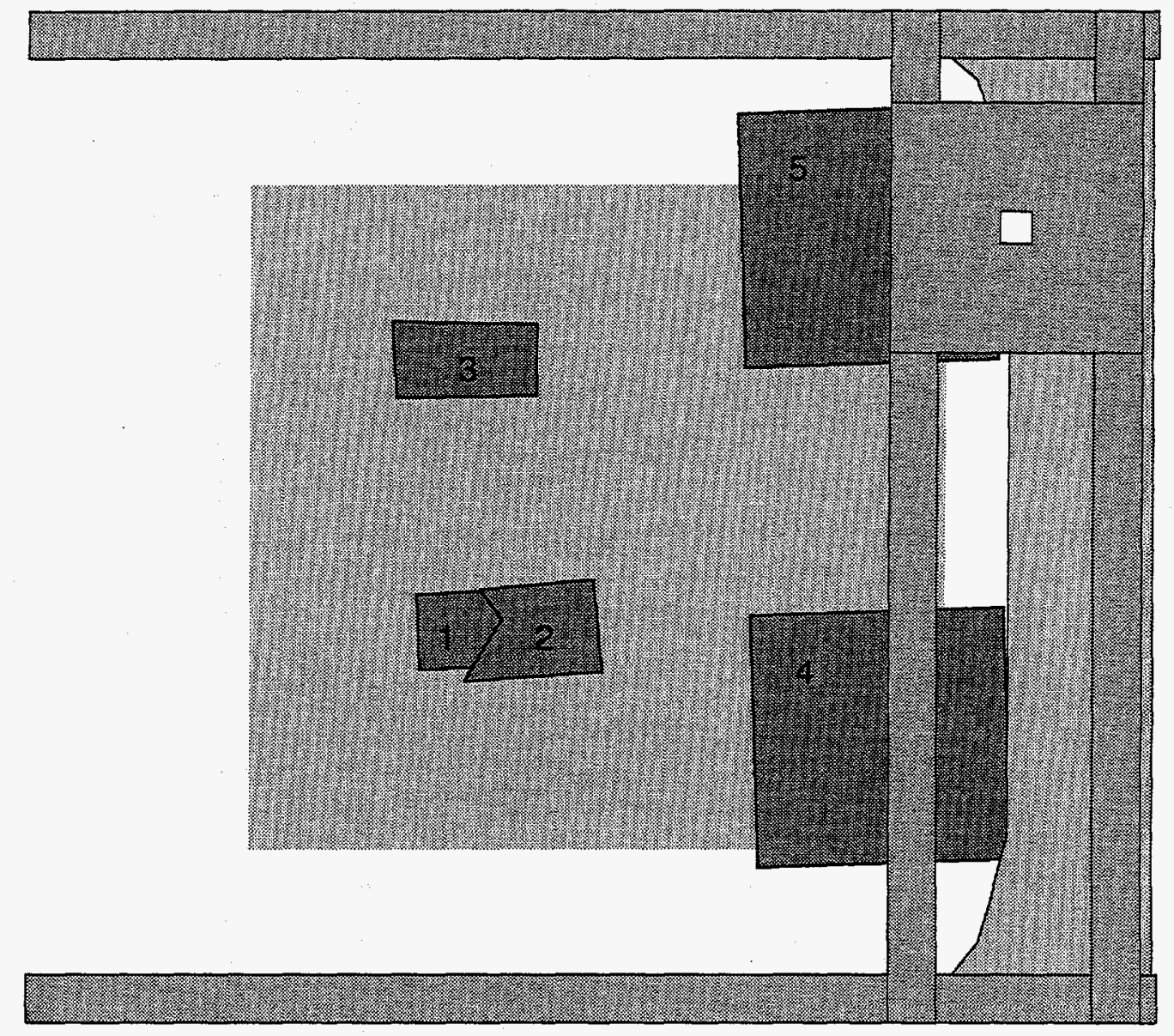

\begin{tabular}{|c|c|c|c|}
\hline Object ID & Description & Depth (ft) & Notes \\
\hline 1 & file cabinet & 2.9 & \\
\hline 2 & file cabinet cover & 2.6 & loose from 1 \\
\hline 3 & file cabinet & 3.0 & \\
\hline 4 & wooden box & 5.4 & contains metal scrap \\
\hline 5 & wooden box & 5.1 & contains wood and paper \\
\hline
\end{tabular}

Figure A-3. Layout of buried objects and the survey scan area relative to the trolley for Experiment 1. 


\section{Experiment 2}

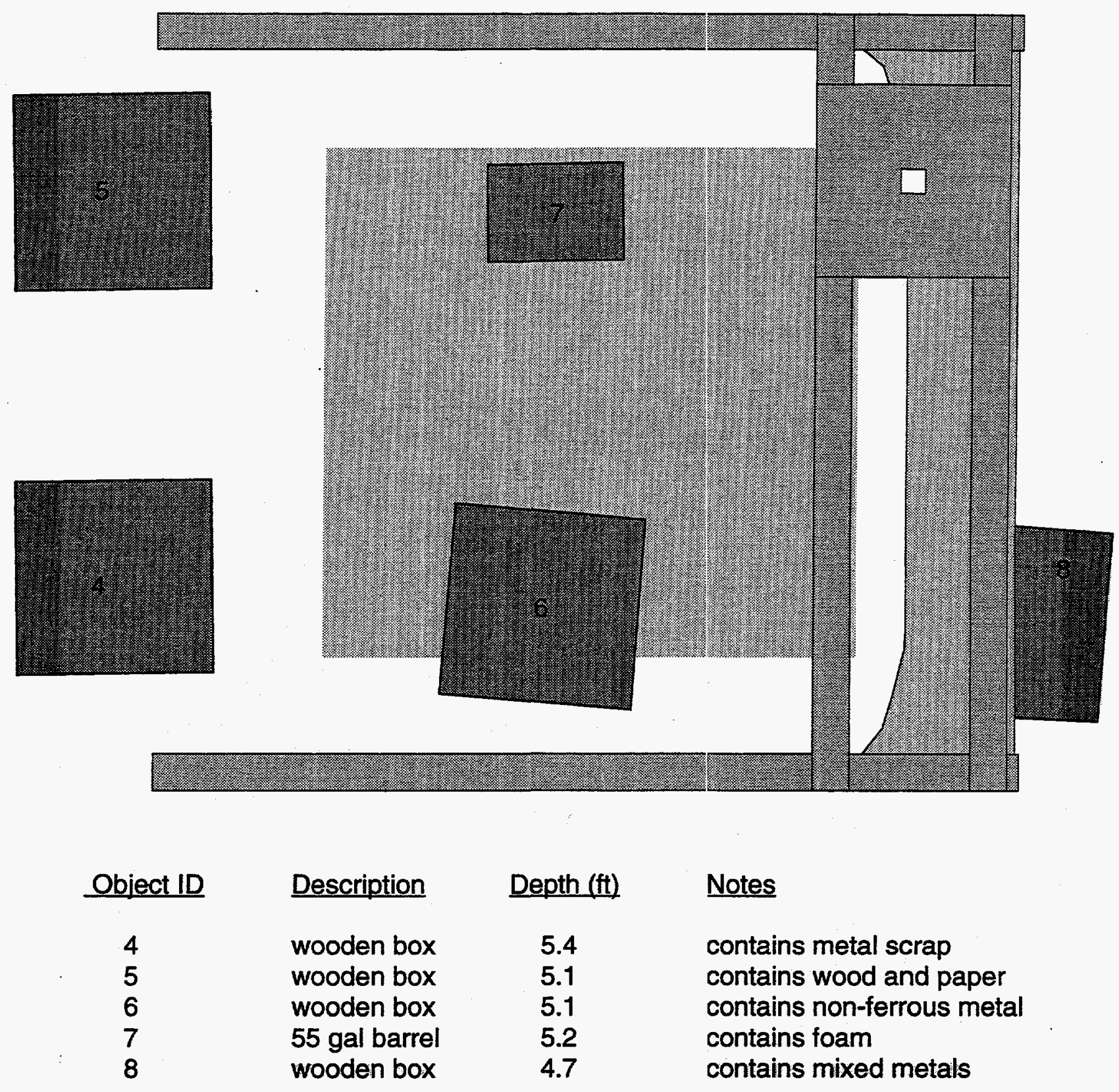

Figure A-4. Layout of buried objects and the survey scan area relative to the trolley for Experiment 2. 


\section{Experiment 3}

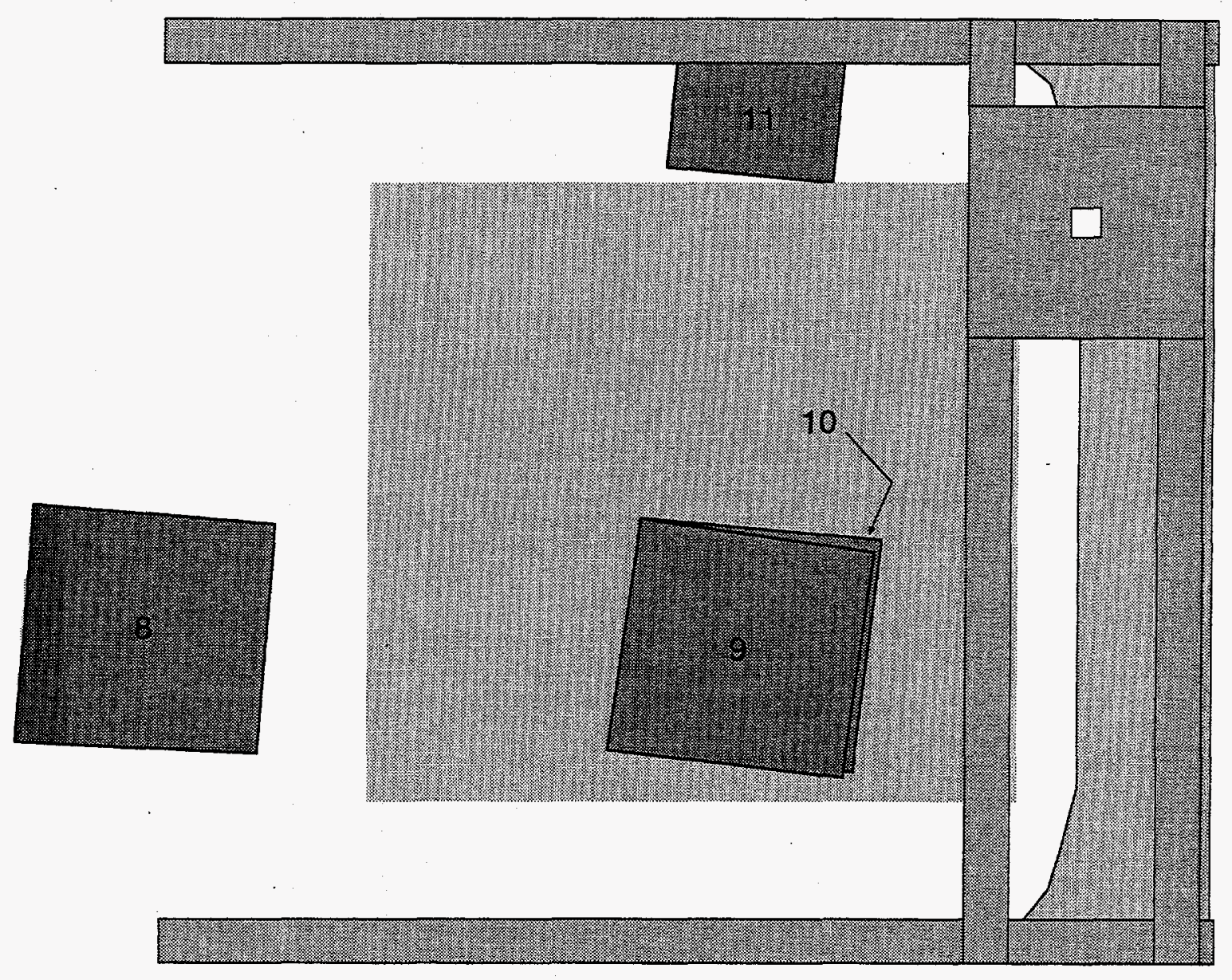

\begin{tabular}{ccccl} 
Object ID & & Description & Depth (ft) & Notes \\
\cline { 1 - 2 } 8 & & wooden box & 4.8 & contains mixed metals \\
9 & wooden box & 2.8 & contains wood and paper \\
10 & wooden box & 4.9 & beneath 9; contains ferrous metal \\
11 & 55 gal barrel & 5.0 & contains concrete
\end{tabular}

Figure A-5. Layout of buried objects and the survey scan area relative to the trolley for Experiment 3. 


\section{Experiment 4}

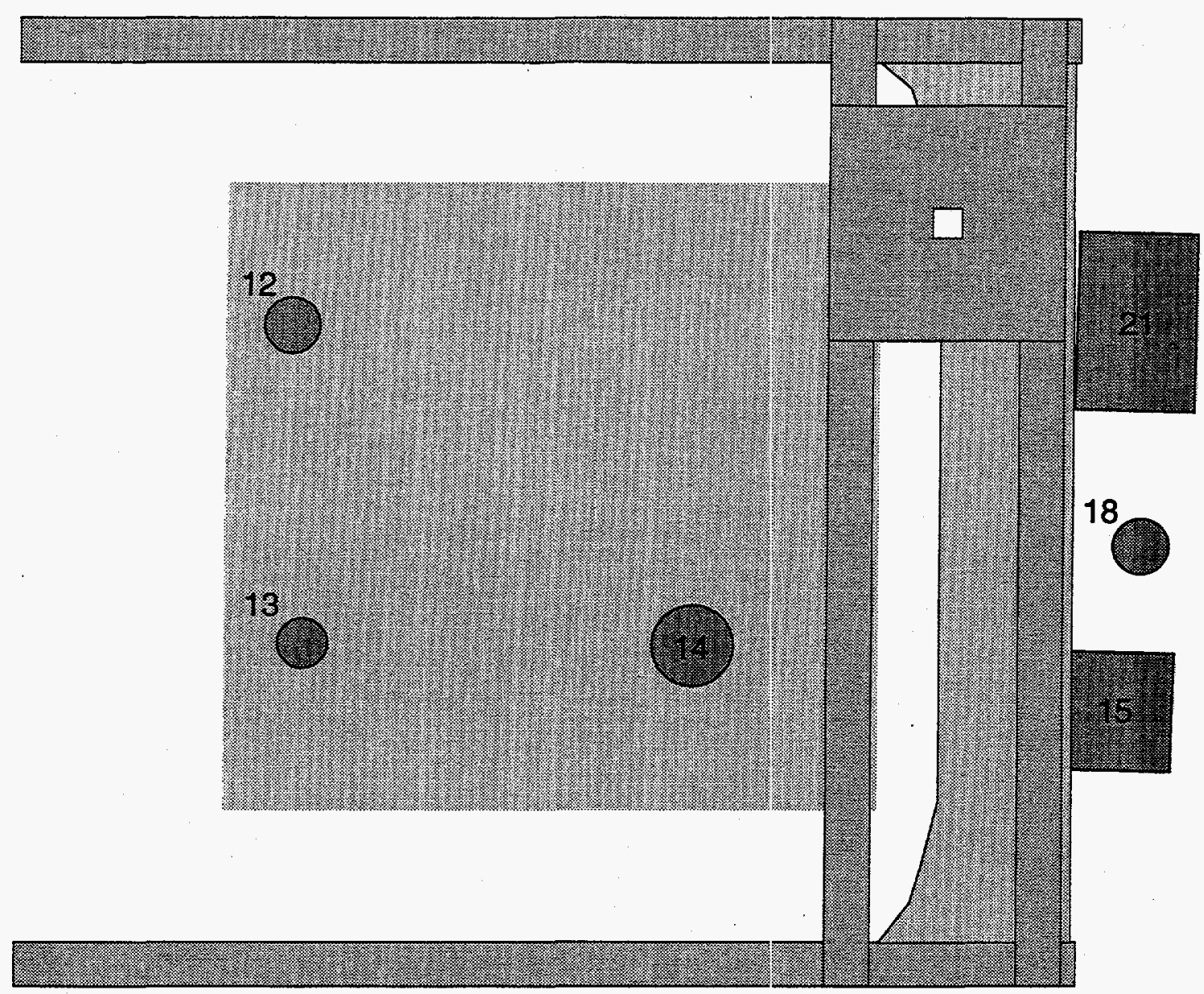

\begin{tabular}{|c|c|c|c|}
\hline Object ID & Description & Depth (ft) & Notes \\
\hline 12 & alcohol plume & 2.4 & $5 \mathrm{gal}$ point source \\
\hline 13 & alcohol plume & 2.4 & $5 \mathrm{gal}$ point source \\
\hline 14 & $\mathrm{NaCl}$ plume & 2.4 & $5 \mathrm{gal}$ \\
\hline 15 & 5 gallon pails & 3.7 & vertical stack, 4 on 4 \\
\hline 18 & 5 gallon pail & 4.8 & vertical, empty \\
\hline 21 & wooden box & 3.6 & empty \\
\hline
\end{tabular}

Figure A-6. Layout of buried objects and the survey scan area relative to the trolley for Experiment 4. 
VOC because of surface air currents and the inability of the VOC sensor to detect organic vapors away from the immediate source.

\section{Experiment 5}

Experiment 5 was conducted over the central part of the Experiment Cell as shown in Figure A-2. This part of the experiment pit contains four wooden boxes, several steel 5-gal pails in stacked and singular configurations, and two steel 55-gal drums in vertical and horizontal orientations. (Figure A-7 contains a detailed drawing of Experiment 5.)

Six levels of magnetic and electromagnetic data were collected over the Experiment 5 area. The EM data included vertical and horizontal dipole quadrature and inphase measurements for a total of 30 distinct surveys. The 55-gal upright barrel (object 20) was removed after the first excavation of approximately 8 in. The uppermost 5 -gal pail on the pyramid stack (object 16) was removed after the second excavation of approximately $6 \mathrm{in}$. Both objects were later replaced after the experiment was complete. Total excavation depth for Experiment 5 was about $1.5 \mathrm{ft}$.

\section{Experiment 6}

Experiment 6 was conducted over the westernmost portion of the Experiment Cell as shown in Figure A-2. (Figure A-8 contains a detailed drawing of Experiment 6.) This part of the cell contains a number of long, linear, metallic objects and some isolated massive objects. These objects include a steel I-beam, copper pipe, an aluminum box beam, a V-8 automobile engine, a large concrete plug, and a short section of thick-walled pipe. Additionally, two 7-ft furrows were dug and filled with isopropyl alcohol and a saturated salt solution respectively. The objective of this action was to determine if contaminant plumes could be differentiated from buried objects.

Seven levels of magnetic and electromagnetic data were collected. The EM data were collected in vertical and horizontal dipole modes for both quadrature and inphase components. Three levels of VOC data were also collected. In total, 38 distinct surveys were conducted and the experiment area was excavated to approximately a $2.5 \mathrm{ft}$ depth. The vertical steel pipe (object 27) was removed after the first excavation of approximately 8 in. It was later replaced after the experiment was complete.

\section{A-2.1.2 Sensor Subsystem}

The digface performance experiments used a passive magnetic field sensor, an active electromagnetic sensor, and an ionization-based chemical sensor. Each of these sensors provided an analog output that was converted to a digital value by combinating analog-to-digital (AD) hardware in the data acquisition system (DAS) system and the proper conversion factors supplied by the sensor manufacturer. Detailed descriptions of each sensor are provided in Appendix A of the test plan (Josten and Roybal 1993). A general description of each sensor is provided below.

A-2.1.2.1 Magnetics. A Electro Mechanical Design Services, Inc. GRS-1 Fluxgate Magnetometer was used to sense the local magnetic field. This instrument is responsive to ferrous material, which can indicate hazardous substances in the area (e.g., hazardous material inside metal containers). The sensor measures the ambient magnetic field along the axis of the magnetometer and the magnetic gradient of the ambient magnetic field aligned with the sensor axis. The instrument is sensitive to orientation because the magnetic field of the Earth is a vector quantity. However, this did not present a problem because the trolley subsystem provided a stable platform for measurements. The magnetic sensor was always oriented vertically during the performance tests. Therefore, the measurements 


\section{Experiment 5}

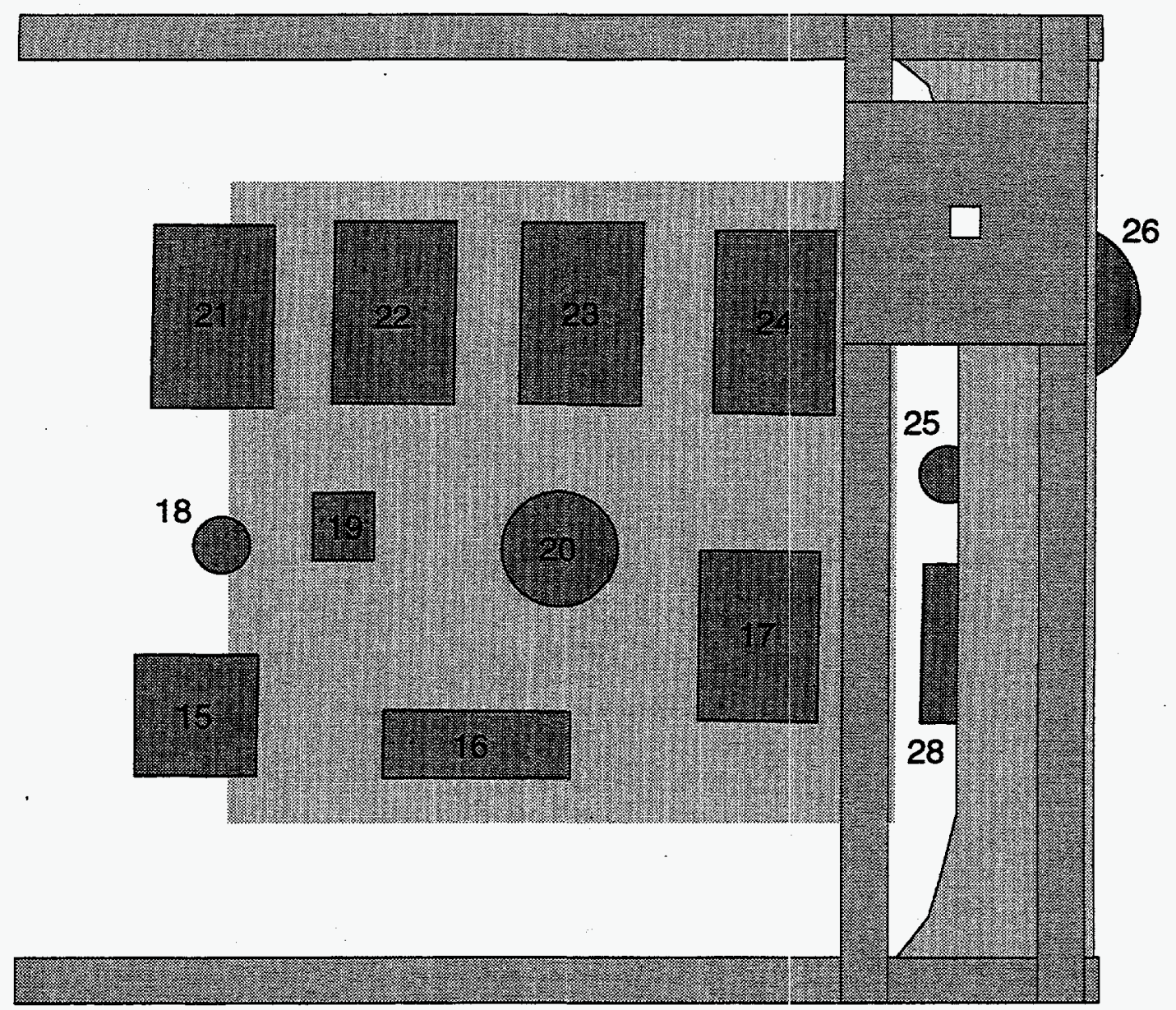

\begin{tabular}{|c|c|c|c|}
\hline Object ID & Description & Depth (ft) & Notes \\
\hline 15 & 5 gallon pails & 3.7 & vertical stack, 4 on 4 \\
\hline 16 & 5 gal pails & 3.4 & pyramid stack, 1-2-3 \\
\hline 17 & 55 gal basrrel & 3.9 & horizontal, empty \\
\hline 18 & 5 gallon pail & 4.8 & vertical, empty \\
\hline 19 & 5 gallon pail & 4.9 & horizontal, empty \\
\hline 20 & 55 gallon barrel & 3.1 & vertical, empty \\
\hline 21 & wooden box & 3.6 & empty \\
\hline 22 & wooden box & 3.7 & contains dry dirt \\
\hline 23 & wooden box & 3.7 & contains wet dirt \\
\hline 24 & wooden box & 3.7 & empty \\
\hline 25 & iron pipe & 4.4 & vertical \\
\hline 26 & concrete block & 3.7 & 6 in. steel loop on top \\
\hline 28 & truck engine & 4.0 & V8 \\
\hline
\end{tabular}

Figure A-7. Layout of buried objects and the survey scan area relative to the trolley for Experiment 5. 


\section{Experiment 6}

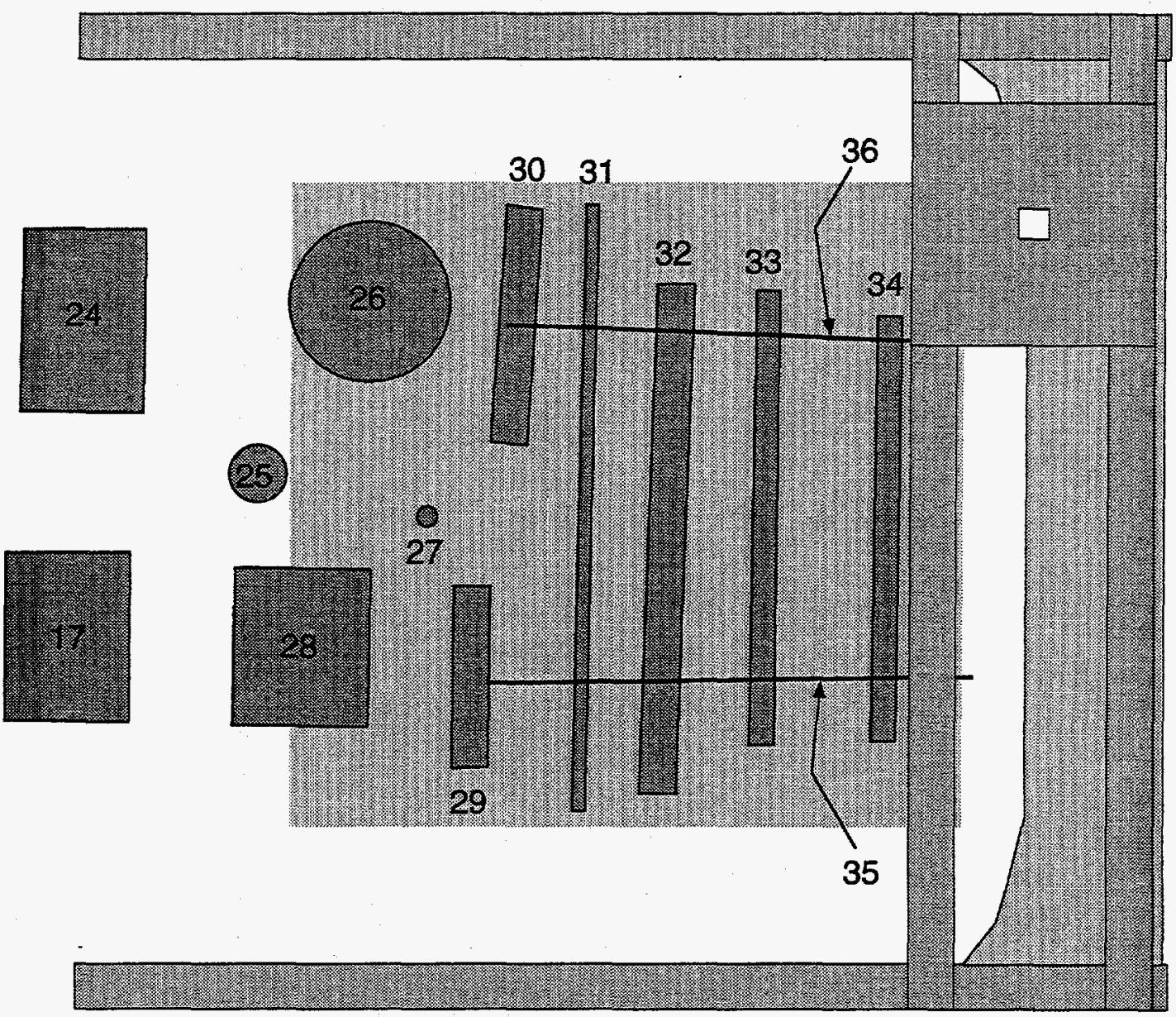

\begin{tabular}{|c|c|c|c|}
\hline Object ID & Description & Depth (ft) & Notes \\
\hline 17 & $55 \mathrm{gal}$ basrrel & 4.0 & horizontal, empty \\
\hline 24 & wooden box & 3.9 & empty \\
\hline 25 & iron pipe & 4.5 & vertical \\
\hline 26 & concrete block & 3.8 & 6 in. steel loop on top \\
\hline 27 & steel pipe & 2.5 & vertical \\
\hline 28 & truck engine & 4.9 & V8 \\
\hline 29 & PVC pipe & 5.3 & horizontal \\
\hline 30 & steel pipe & 5.4 & horizontal \\
\hline 31 & copper bar & 4.8 & \\
\hline 32 & iron " $\mathrm{C}$ " channel & 4.1 & \\
\hline 33 & aluminum bar & 5.2 & \\
\hline 34 & steel bar & 5.8 & \\
\hline 35 & alcohol plume & 2.4 & 15 gal poured in trench \\
\hline 36 & $\mathrm{NaCl}$ plume & 2.4 & 15 gal poured in trench \\
\hline
\end{tabular}

Figure A-8. Layout of buried objects and the survey scan area relative to the trolley for Experiment 6 . 
performed with the magnetometer amounted to determining the vertical field and the vertical gradient of the vertical field.

The GRS-1 magnetometer has stated sensitivities of 20,000 nanoTesla (nT) per volt for a vector field measurement and $32,808 \mathrm{nT} / \mathrm{m}$ per volt for a gradient measurement. These sensitivities combined with a $\mathrm{AD}$ range of $\pm 5 \mathrm{~V}$ and 18-bit effective resolution provide resolution capabilities of $0.8 \mathrm{nT}$ for the vector field and $1.25 \mathrm{nT} / \mathrm{m}$ for the vector gradient.

A-2.1.2.2 Electromagnetics. A Geonics, Ltd. EM38 Ground Conductivity Meter was used to measure quadrature and inphase components of induced magnetic fields as a percentage of a transmitted primary magnetic field. This sensor responds to the presence of electrically conductive metals and ground conductivity changes potentially caused by fluids in the ground. The EM38 has a linear geometry consisting of a transmitter and receiver pair of coils that are approximately $1 \mathrm{~m}$ apart. Because of this geometry, the instrument is also orientation sensitive. The sensor was always aligned parallel to the trolley $y$-axis for the performance tests.

The instrument produces a differential output that requires the DAS to subtract the two signals generated by the EM38. The quadrature (or apparent conductivity) output from the sensor has a stated sensitivity of 4,000 milliSiemen per meter per volt and the inphase output has a sensitivity of 115 part per thousand per volt. Unlike other Geonics, Ltd. conductivity devices, the EM-38 does not produce quadrature and inphase outputs simultaneously requiring two separate pad surveys to obtain this information.

The depth of investigation of the EM38 may be changed by changing the coil orientation from vertical to horizontal. Thus, four data collection modes are possible: horizontal dipole quadrature (EM-Q-H), horizontal dipole inphase (EM-IP-H), vertical dipole quadrature (EM-Q-V), and vertical dipole inphase (EM-IP-V). These designations are used throughout the results discussion in the next section.

A-2.1.2.3 VOCs. A HNU Systems, Inc. DL-101 instrument was used to sense volatile organic material placed in simulated plumes at the digface. This instrument uses photo ionization as the detection principle and has an effective range of 0.1 to 2,000 parts per million (ppm) for benzene, which is the preferred calibration fluid. Isopropyl alcohol was used as the organic for environmental reasons, and appropriate calibration procedures were established for the alcohol. The sensor incorporates a small vacuum pump to draw ambient air into the ionization chamber and consequently has a slow response time ( $<3$ seconds to $90 \%$ response as per the manufacturer). The instrument provides an analog output that is measured by the DAS. Stated sensitivity is $1,000 \mathrm{ppm}$ per volt.

\section{A-2.2 Summary of Data Collection}

The performance tests generated over 200,000 independent sensor measurements. The position of each raw measurement was recorded based on a local coordinate system tied to the trolley structure. The position data have subsequently been transformed into Idaho State Plane coordinates providing a world coordinate system that ties together all the data as well as the locations of objects within the pits.

For the magnetometer and EM38, the number of data collection levels ranged from 4 (for Experiment 1) to 9 (for Experiment 3). These data were collected at 0.24 in. intervals in the $x$ direction, and $0.5 \mathrm{in}$. intervals in the $\mathrm{y}$ and $\mathrm{z}$ directions. HNU data were collected at 0.24 in. intervals in the $\mathrm{x}$ direction and 12 in. intervals in the y direction. For the HNU, the number of levels and the z-spacing 
between levels varied depending on the elevation of the digface after each excavation step. Table A-3 summarizes data collection for the entire performance testing activity.

Table A-3. Summary of data collection for the FY-93 digface performance tests.

\begin{tabular}{|c|c|c|c|c|c|c|c|}
\hline \multirow[b]{2}{*}{ Sensor } & \multicolumn{7}{|c|}{ Experiment number } \\
\hline & 1 & 2 & 3 & 4 & 5 & 6 & Total \\
\hline \multicolumn{8}{|l|}{ Total data levels collected } \\
\hline Magnetic vertical field & 4 & 9 & 8 & - & 6 & 7 & 34 \\
\hline Magnetic vertical gradient & 4 & 9 & 8 & - & 6 & 7 & 34 \\
\hline EM-Q-H & 4 & 9 & 8 & 6 & 6 & 7 & 40 \\
\hline EM-IP-H & 4 & 9 & 8 & - & 6 & 7 & 34 \\
\hline EM-Q-V & - & 9 & 8 & 3 & 6 & 6 & 32 \\
\hline EM-IP-V & - & 9 & 8 & - & 6 & 6 & 29 \\
\hline $\mathrm{HNU}$ & - & - & - & 3 & - & 3 & 6 \\
\hline Total & 16 & 54 & 48 & 12 & 36 . & 43 & 209 \\
\hline \multicolumn{8}{|l|}{ Total data points collected } \\
\hline Magnetic vertical field & 4,350 & 9,900 & 8,800 & - & 6,600 & 7,700 & $\mathbf{3 7 , 3 5 0}$ \\
\hline Magnetic vertical gradient & 4,350 & 9,900 & 8,800 & - & 6,600 & 7,700 & 37,350 \\
\hline EM-Q-H & 4,048 & 9,108 & 8,096 & 6,072 & 6,512 & 7,612 & 41,448 \\
\hline EM-IP-H & 4,048 & 9,108 & 8,096 & - & 6,512 & 7,612 & 35,376 \\
\hline EM-Q-V & - & 9,108 & 8,096 & 3,036 & 6,512 & 6,512 & 33,264 \\
\hline EM-IP-V & - & 9,108 & 8,096 & - & 6,512 & 6,512 & 30,228 \\
\hline $\mathrm{HNU}$ & - & - & - & 2,496 & - & 1,716 & 4,212 \\
\hline Total & 16,796 & 56,232 & 49,984 & 11,604 & 39,248 & 45,364 & 219,228 \\
\hline
\end{tabular}


Appendix B

Characterization Cell VREAM Model Screen Images 
B-2 


\section{Appendix B}

\section{Characterization Cell VREAM Model Screen Images}

The Characterization Cell at the Idaho National Engineering Laboratory Radioactive Waste Management Complex is an engineered waste burial site used for development and testing of sensors. Details of the Characterization Cell are given in Appendix A. This appendix provides additional computer screen images showing some of the characteristics of the VREAM model constructed of the Characterization Cell.

Figure B-1 shows a screen dump of the VREAM model of the Characterization Cell as viewed from above and to the south of the cell. Cylinders and rectangular: parallelepipeds represent the buried waste forms (boxes, barrels, and file cabinets) described in Appendix A. The two tall rectangular structures on the right-hand side of the figure are the metal file cabinets. The facsimile of a hand in the figure represents the device with which one can interact with this model.

Figure B-2 is a screen dump of the same model as the user has rotated clockwise about 45 degrees around the south side of the cell. The two stacked boxies appear on the left-hand side of the image.

Figure B-3 shows the viewpoint after the user has rotated counterclockwise approximately 45 degrees more and moved downward. At this position, the viewpoint is just above the floor level of the Characterization Cell. Visible in the foreground are the stacked boxes (objects 9 and 10) and a barrel (object 11).

Figure B-4 shows basically the same view as is Figure B-3 except the user has grabbed the barrel object (object 11 (also known as object $\mathrm{A}$ ) with the hand causing an information window to display in the upper right-hand corner of the screen. The information window provides identification and contents information about the object. Note that the object changed color in response to the grab action.

Figures B-5 and B-6 show the result of the user moving to the left relative to the view in Figure B-4 and then grabbing the top box of the two stacked boxed [object 9 (also known as E1)], respectively.

Figures B-7 and B-8 show the result of the user moving into the scene (traveling east) and then eventually grabbing the left-hand file cabinet [object 1 (also known as F1)], respectively. 


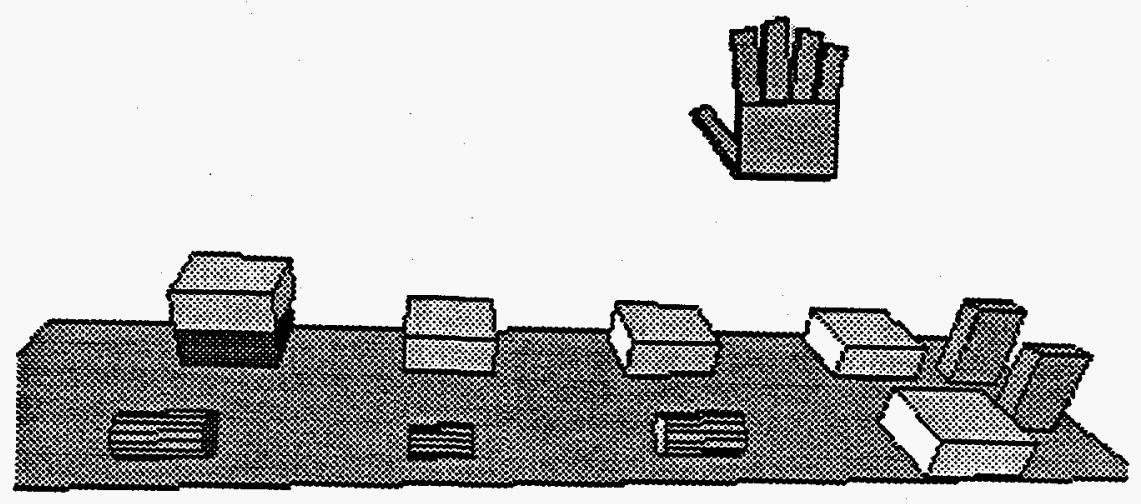

Figure B-1. Screen image of VREAM model view of the Characterization Cell viewed from south to north.

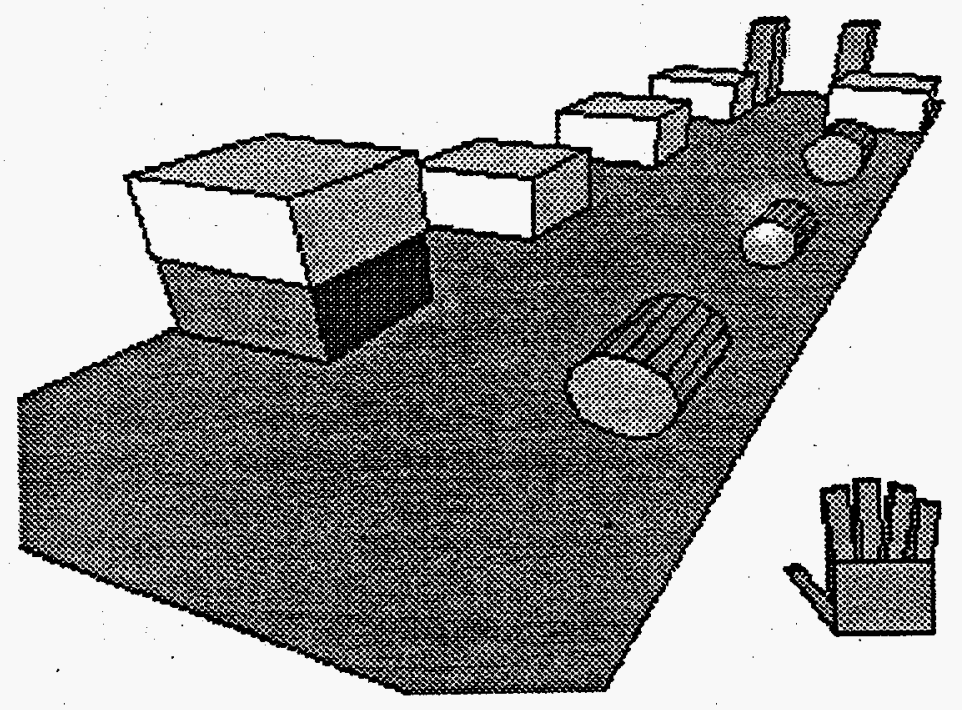

Figure B-2. Screen image of VREAM model view of the Characterization Cell viewed from southwest to northeast. 


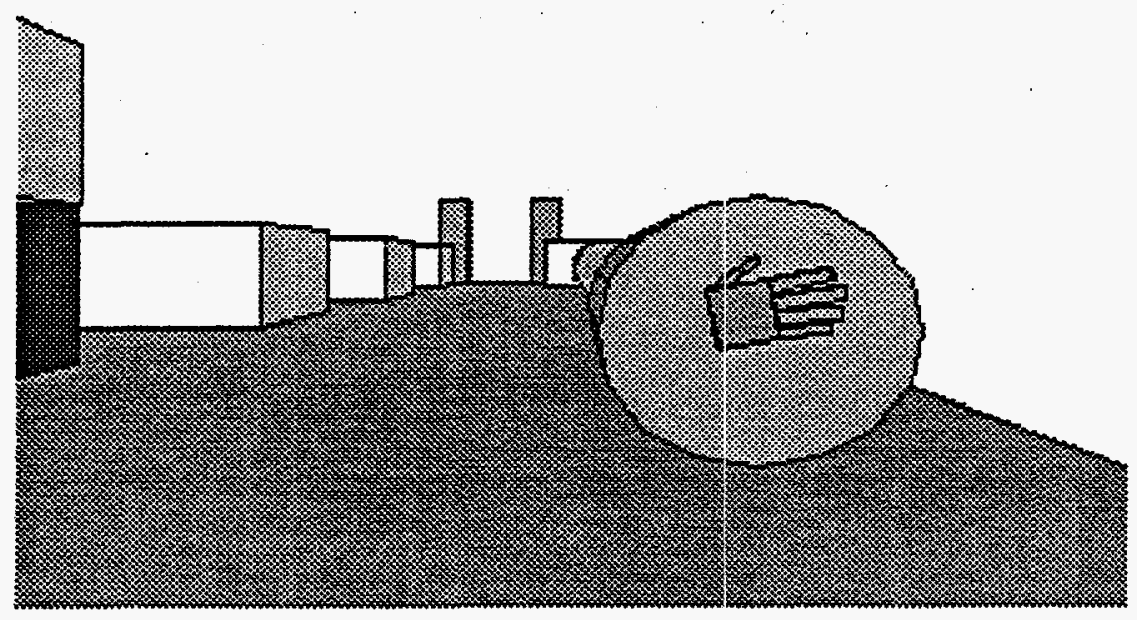

Figure B-3. Screen image of VREAM model view of the Characterization Cell viewed from Cell floor level looking west to east.

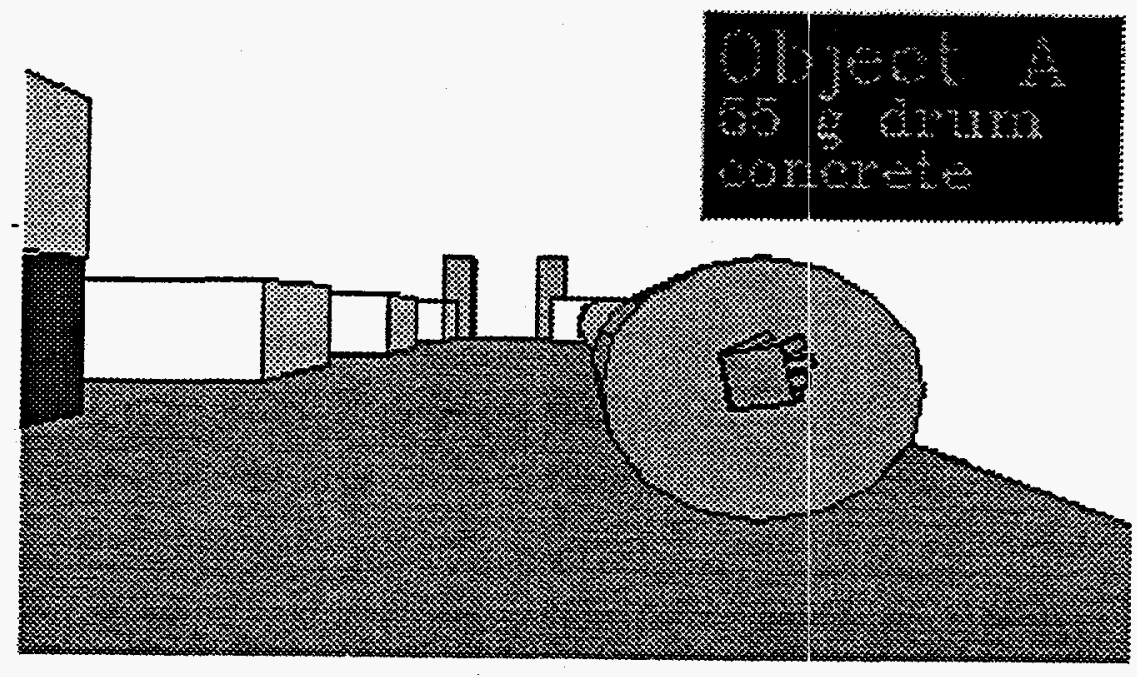

Figure B-4. Screen image of VREAM model view of the Characterization Cell viewed from cell floor level looking west to east as user "grabs" object 11 . 


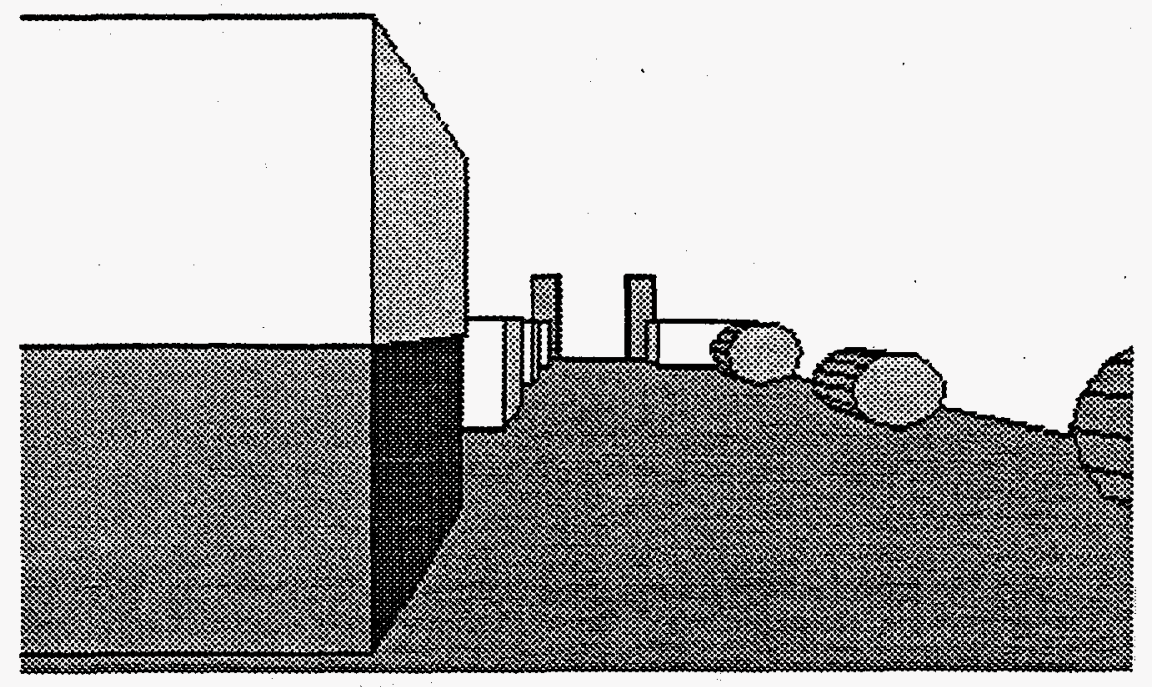

Figure B-5. Screen image of VREAM model view of the Characterization Cell viewed from cell floor level looking west to east.

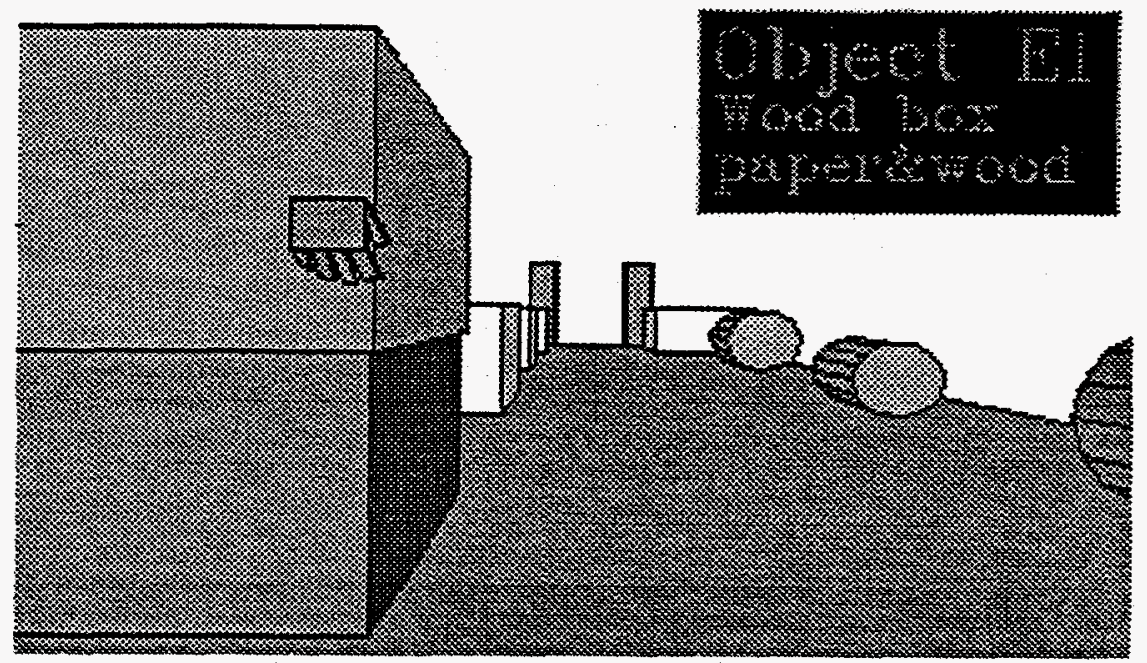

Figure B-6. Screen image of VREAM model view of the Characterization Cell viewed from cell floor level looking west to east. 


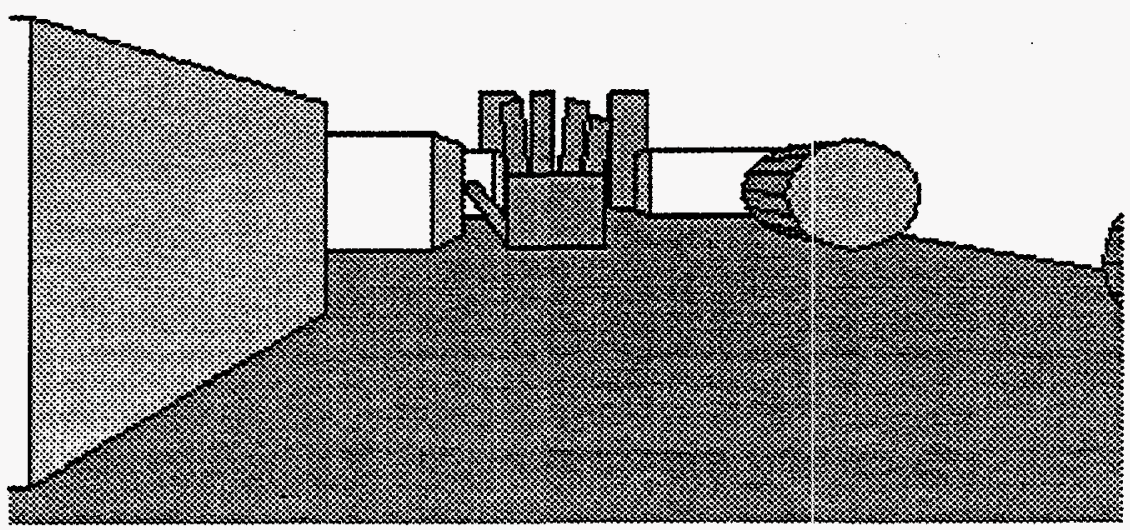

Figure B-7. Screen image of VREAM model view of the Characterization Cell viewed from cell floor level looking west to east.

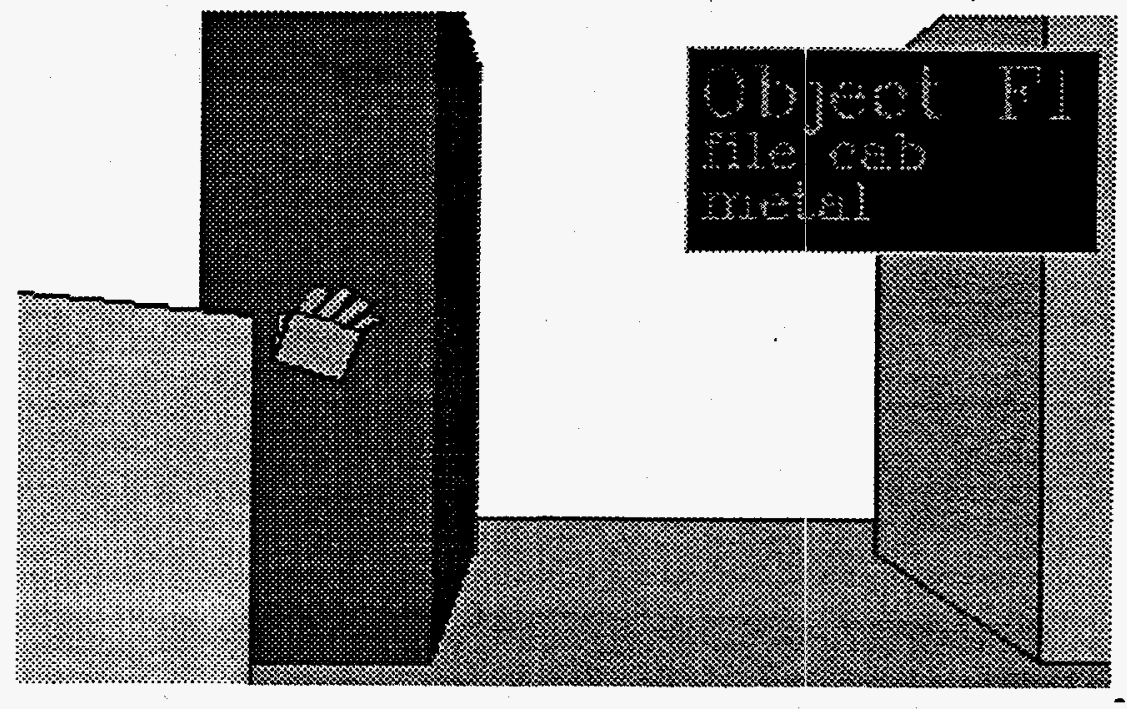

Figure B-8. Screen image of VREAM model view of the Characterization Cell viewed from cell floor level looking west to east. 
Appendix C

Neural Network Supplemental Information 
C-2 


\section{Appendix C}

\section{Neural Network Supplemental Information}

\section{C-1. SUMMARY}

Artificial neural network output for target versus background identification consisted of a file containing a vector of the responses. To evaluate these responses, criteria are needed. Target criteria grids used for electromagnetic (EM) evaluation are presented below $C$ language source code for a trained network.

\section{C-2. TARGET CRITER\|A}

Grids of the target criteria, established for all EM and EM plus magnetic data combined for an experiment (referred to as total or combined) tests, are shown in Figures C-1 through C-6. These grids were generated based on the target location information given in Appendix A. The networks used these locations to compare their outputs and determine the classification rate and root mean square values that represent this comparison. The lighter regions are targets with a value of +1 , . while the darker area is "background" with a value of -1 . The ring features surrounding the "targets" are features added by the gridding program. E1-CRITERIA is the grid for Experiment 1 criteria, E2-CRITERIA is for Experiment 2, and so on. These grids were used to compare the network output grids and to ensure that all desired targets were used in the criteria.

\section{C-3. EXAMPLE SOURCE CODE}

An example for a neural network trained using the data from digface Experiment 3, level 7 is shown below. C language source code can be generated by the neural network development program (NeuralWare) used for the neural network examination of the digface data. This allows one to embed the neural network into a larger framework if desired. The particular network used here for illustration was trained and evaluated using the EM sensor (sensor: 2, 3, 4, and 5) data for level 7 of Experiment 7. Input required for the network is basically a concatenated file consisting of the $\mathrm{x}, \mathrm{y}, \mathrm{z}$ coordinates and all the EM data. Table C-1 shows a section of the input file. Output from network is written to a file consisting of the coordinate positions and network output. A portion of the output generated by the network is shown in Table C-2. As mentioned above, an output of -1 (or less) indicates background and an output of +1 (or above) indicates target.

Table C-3 shows the $\mathrm{C}$ language source code for this neural network. 


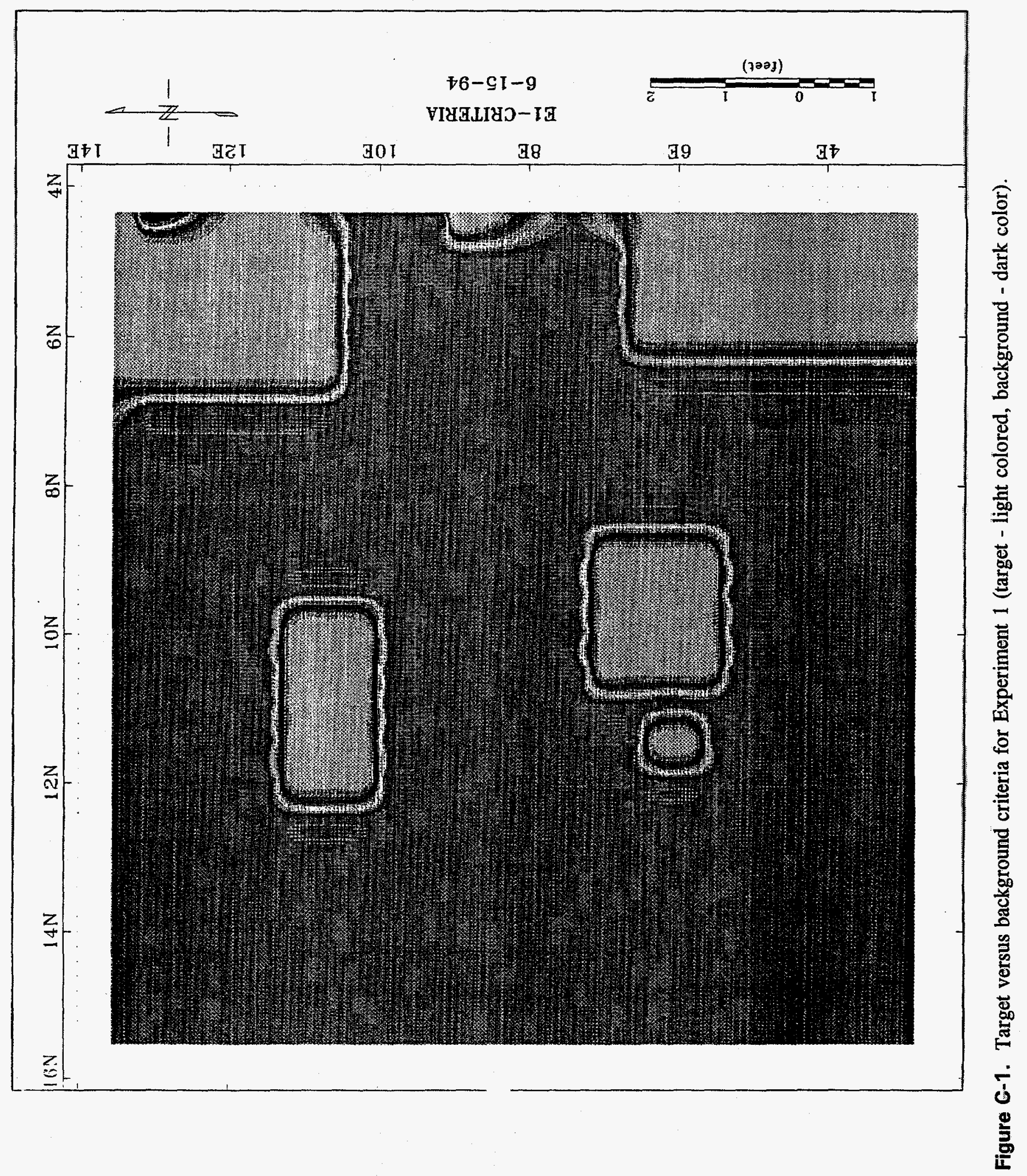




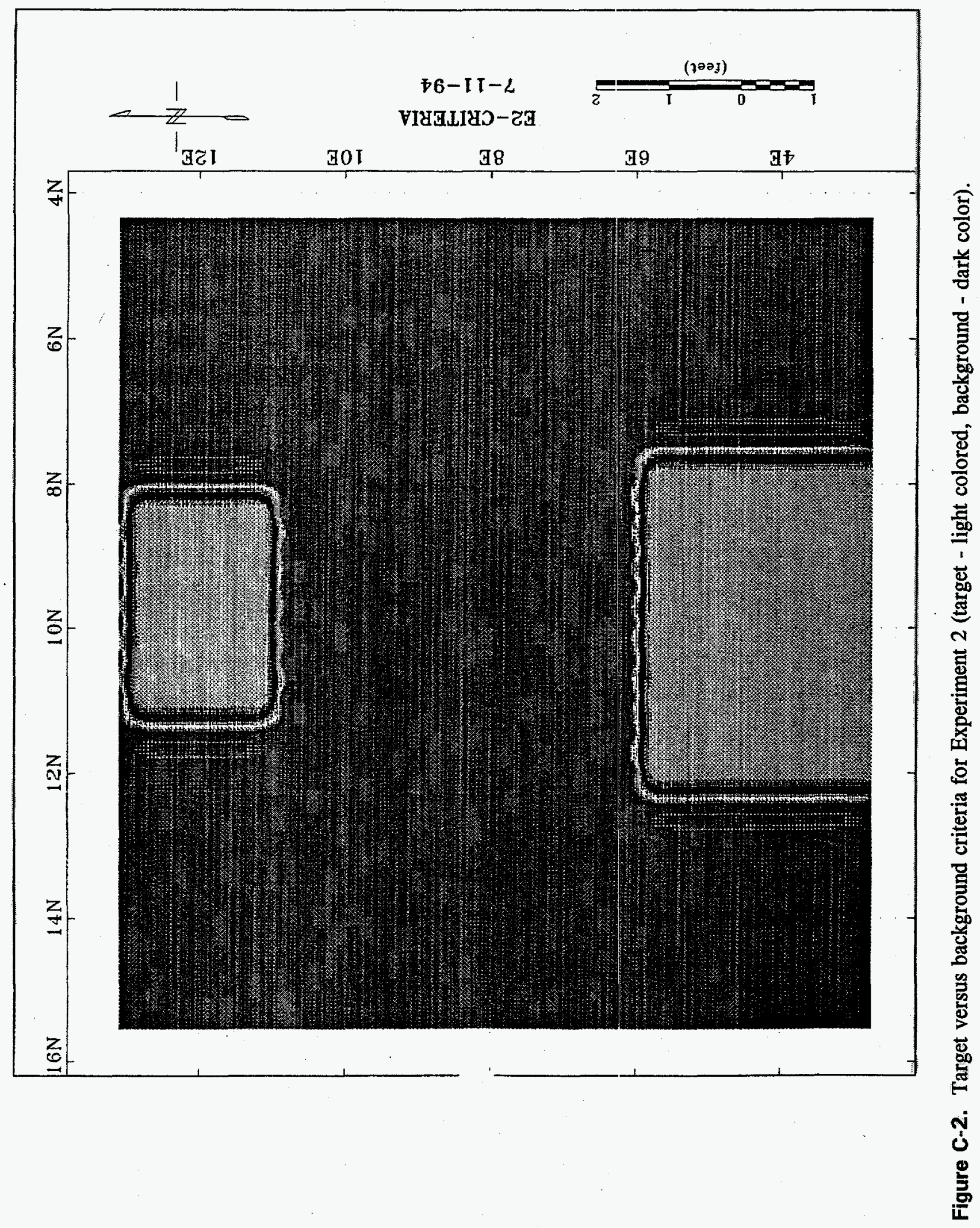




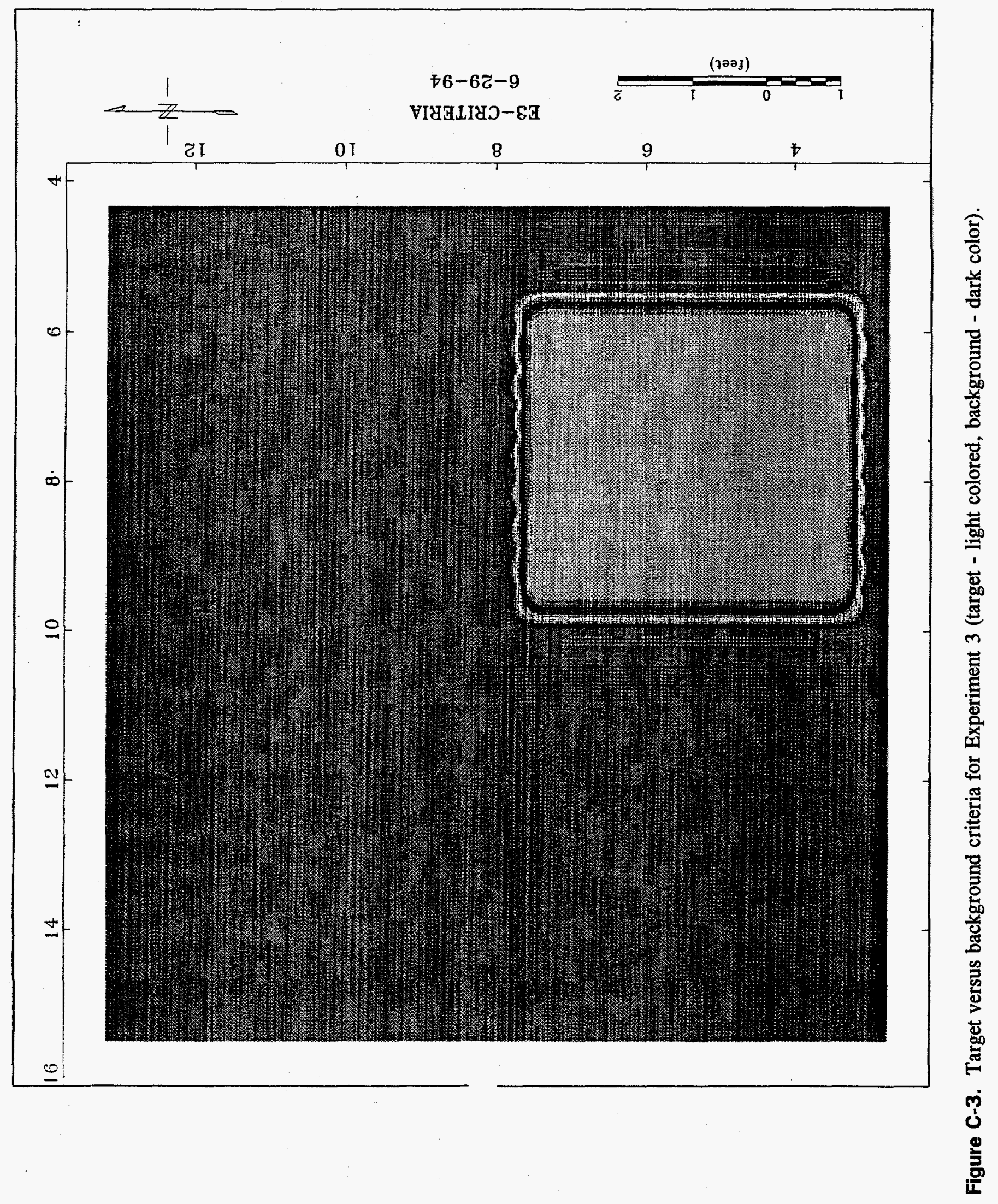

C-6 


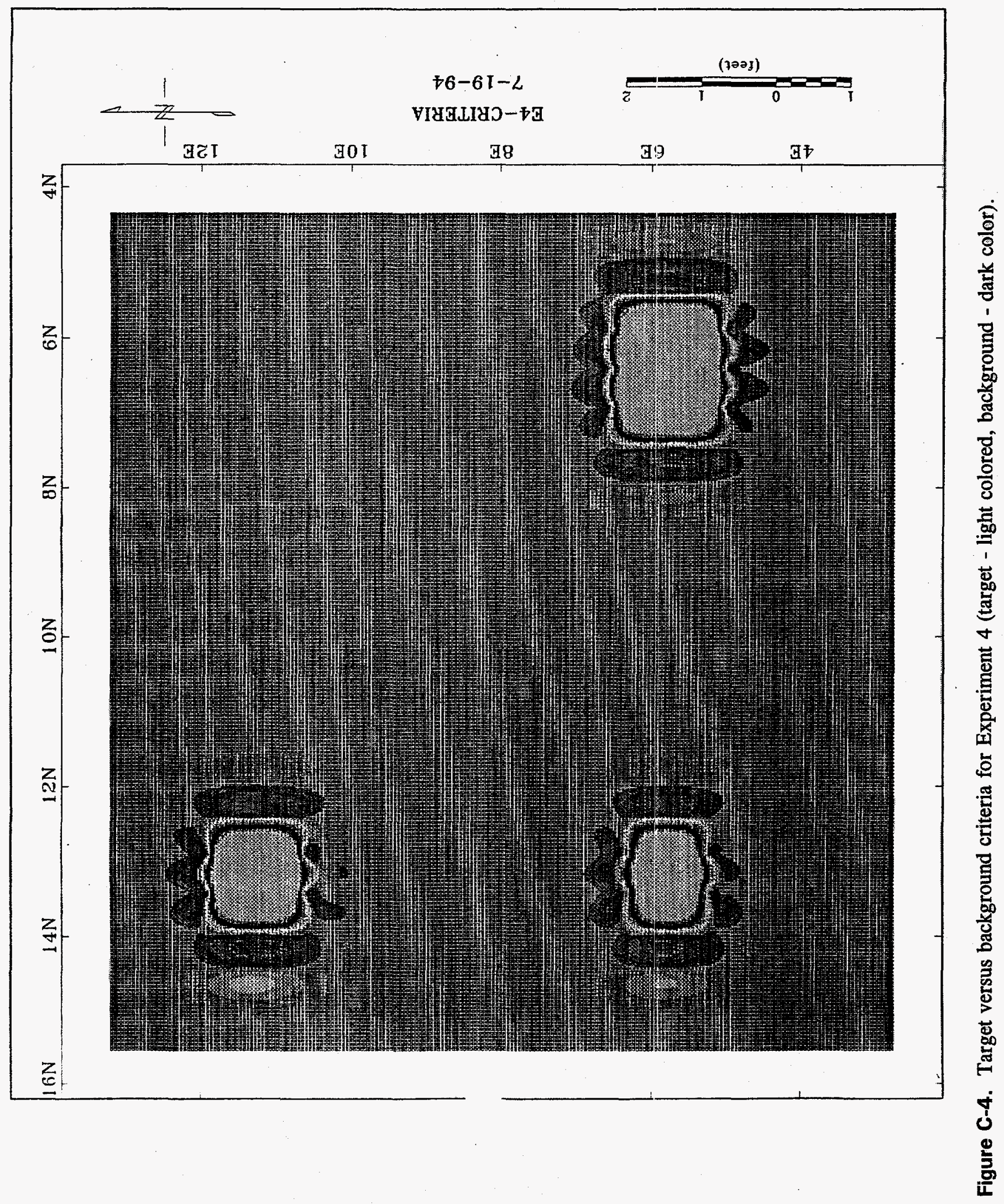




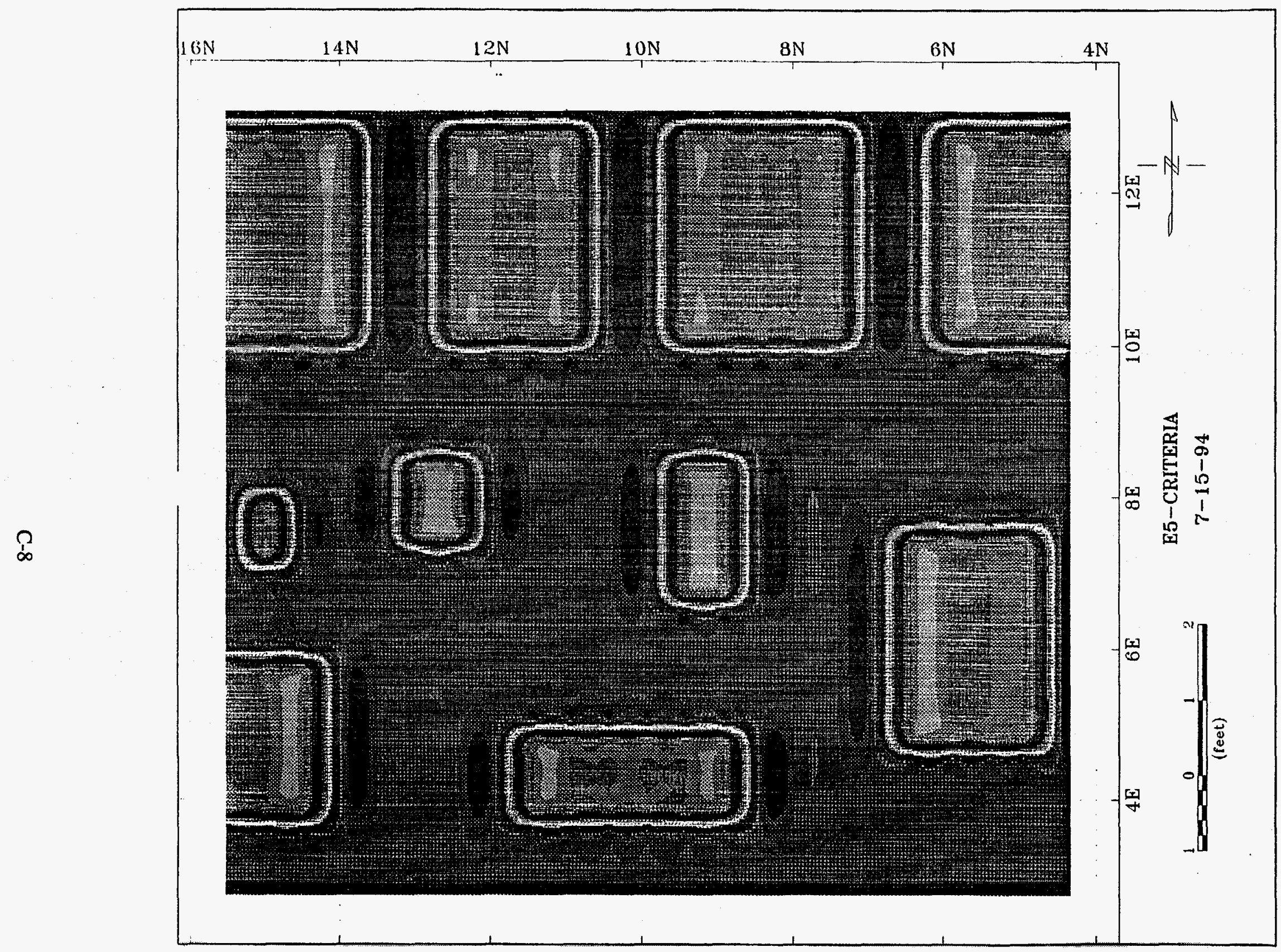

Figure C-5. Target versus background criteria for Experiment 5 (target - light colored, background - dark color). 


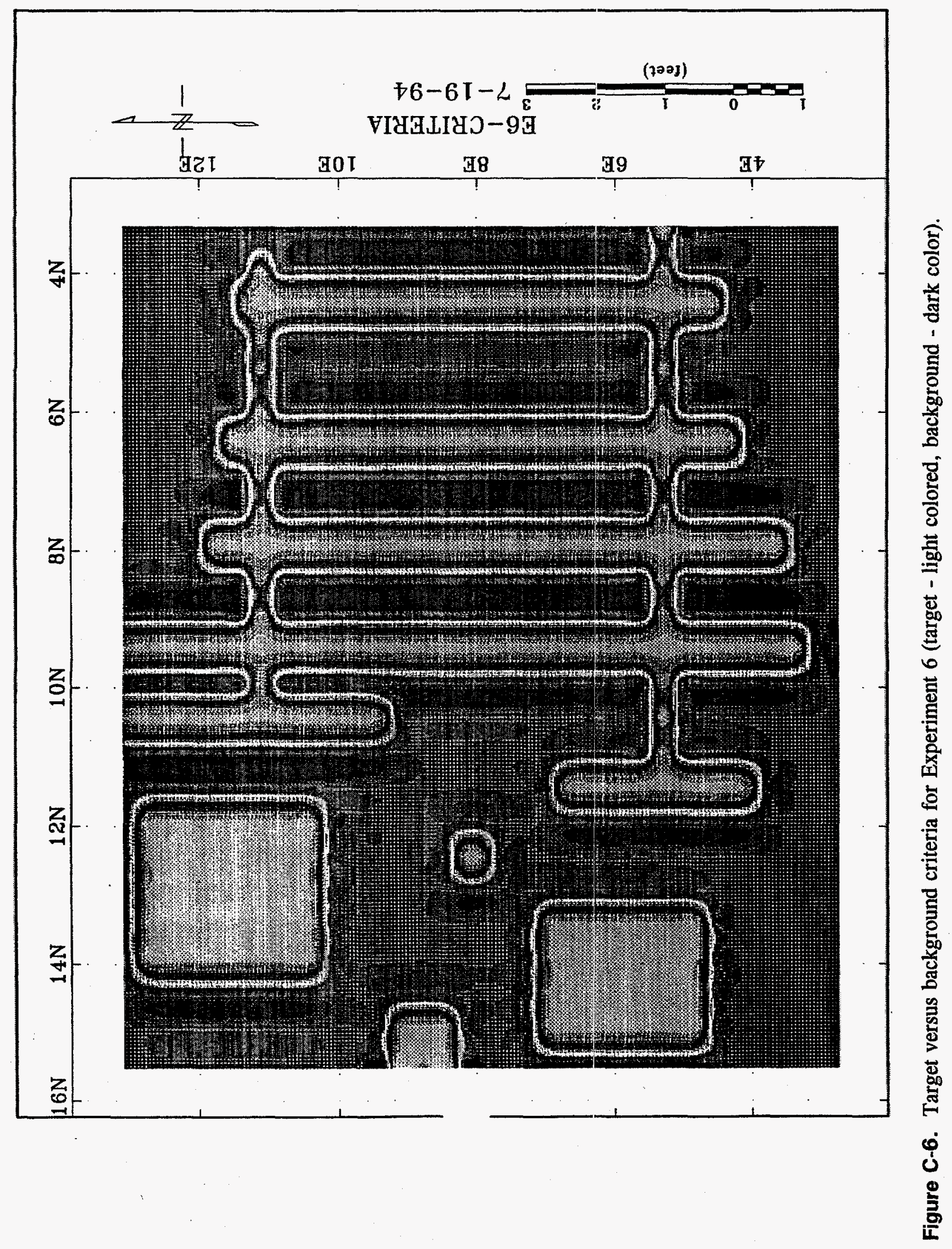

C-9 
Table C-1. Input for example neural network.

\begin{tabular}{|lcccccc|}
\hline$\underline{\mathrm{x}}$ & $\underline{\mathrm{y}}$ & $\underline{\mathrm{z}}$ & $\underline{\text { Sensor 2 }}$ & $\underline{\text { Sensor 3 }}$ & $\underline{\text { Sensor 4 }}$ & $\underline{\text { Sensor 5 }}$ \\
2.86 & 4.44 & -4.08 & 146 & 9 & 103.3 & -0.6 \\
3.12 & 4.44 & -4.08 & 256.4 & 12.3 & 157.4 & 1.7 \\
3.35 & 4.44 & -4.08 & 225.5 & 11.1 & 121.6 & -1.9 \\
3.6 & 4.44 & -4.08 & 220 & 10.6 & 101.5 & -4.9 \\
$\ldots \ldots \ldots$ & & & & & & \\
\hline
\end{tabular}

Table C-2. Output for example neural network.

\begin{tabular}{|cccr|}
\hline$\underline{x}$ & $\underline{y}$ & $\underline{z}$ & Net output \\
13.040000 & 6.430000 & -4.080000 & \\
2.860000 & 6.930000 & -4.080000 & -1.064853 \\
3.110000 & 6.930000 & -4.080000 & -0.730877 \\
3.350000 & 6.930000 & -4.080000 & -0.282556 \\
3.590000 & 6.930000 & -4.080000 & 0.852686 \\
3.830000 & 6.930000 & -4.080000 & 0.979544 \\
\hline
\end{tabular}


Table C-3. C language source code for Experiment 3, level 7 magnetic network.

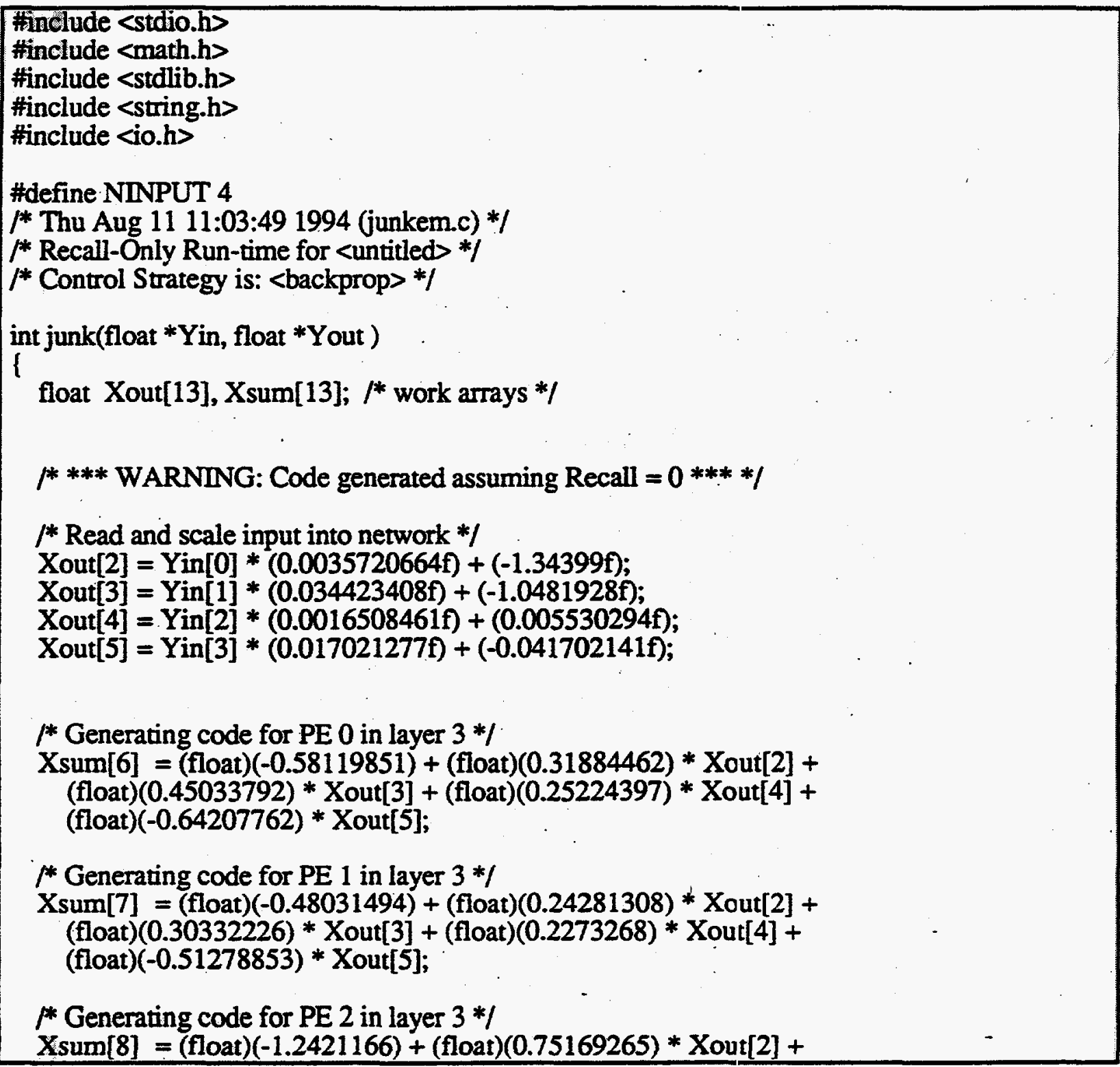


Table C-3. (continued).

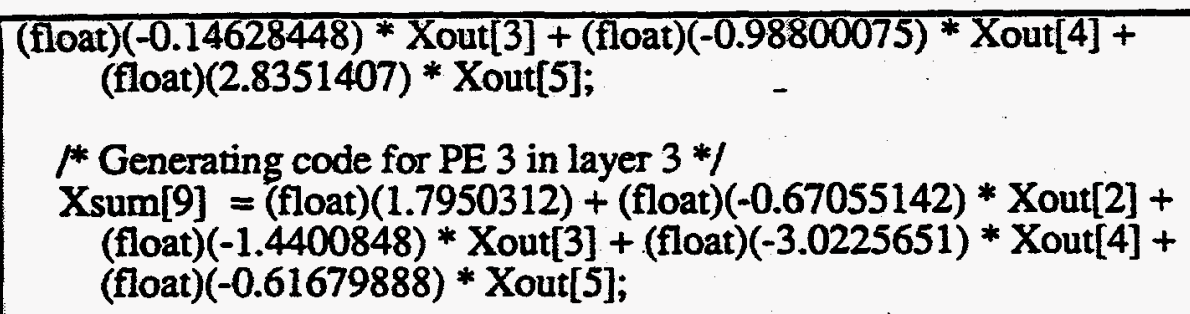

* Generating code for PE 4 in layer $3 *$ /

$X \operatorname{sum}[10]=($ float $)(-0.86769587)+($ float $)(2.8093309) *$ Xout $[2]+$ (float) $(2.8338015) *$ Xout [3] $+($ float $)(-1.808522) *$ Xout $[4]+$ (float) $(0.69450641) *$ Xout[5];

* Generating code for PE 5 in layer $3 *$ /
Xsum $[11]=($ float $)(-1.4719238)+($ float $)(6.7552209) *$ Xout $[2]+$ (float) $(3.5697625) *$ Xout $[3]+($ float $)(-1.9011079) *$ Xout $[4]+$ (float) $(-2.1230559) *$ Xout $[5]$;

$1 *$ Generating code for PE 0 in layer $3 * 1$

Xout[6] = (float) $\tanh ($ (double) Xsum[6] ;

* Generating code for PE 1 in layer $3 *$ f

Xout[7] = (float) tanh ( (double) Xsum[7] );

* Generating code for PE 2 in layer $3 *$ l

Xout $[8]=$ (float) $\tanh ($ (double) Xsum[8] );

* Generating code for PE 3 in layer $3 *$

Xout[9] $=$ (float) $\tanh ($ (double) Xsum[9] );

$f^{*}$ Generating code for PE 4 in layer $3 *$ I

Xout $[10]=$ (float) $\tanh ($ (double) Xsum[10] );

* Generating code for PE 5 in layer $3 *$ I

Xout[11] = (float) $\tanh ($ (double) Xsum[11] );

* Generating code for PE 0 in layer $4 *$ I

Xsum[12] $=($ float $)(-0.078371227)+($ float $)(-0.15887693) *$ Xout $[6]+$ (float) $(-0.10108677) *$ Xout $[7]+($ float $)(1.5689932) *$ Xout $[8]+$ (float) $(1.6149535) *$ Xout $[9]+($ float $)(0.60079795) *$ Xout $[10]+$ (float) $(0.73317903) *$ Xout $[11]$;

Xout $[12]=$ (float) $\tanh ($ (double) Xsum[12] );

/* De-scale and write output from network */

Yout $[0]=$ Xout $[12] *(1.25 f)$

*. Generating code for PE 0 in layer $4 * /$

return( 0 ); 
Table C-3. (continued).

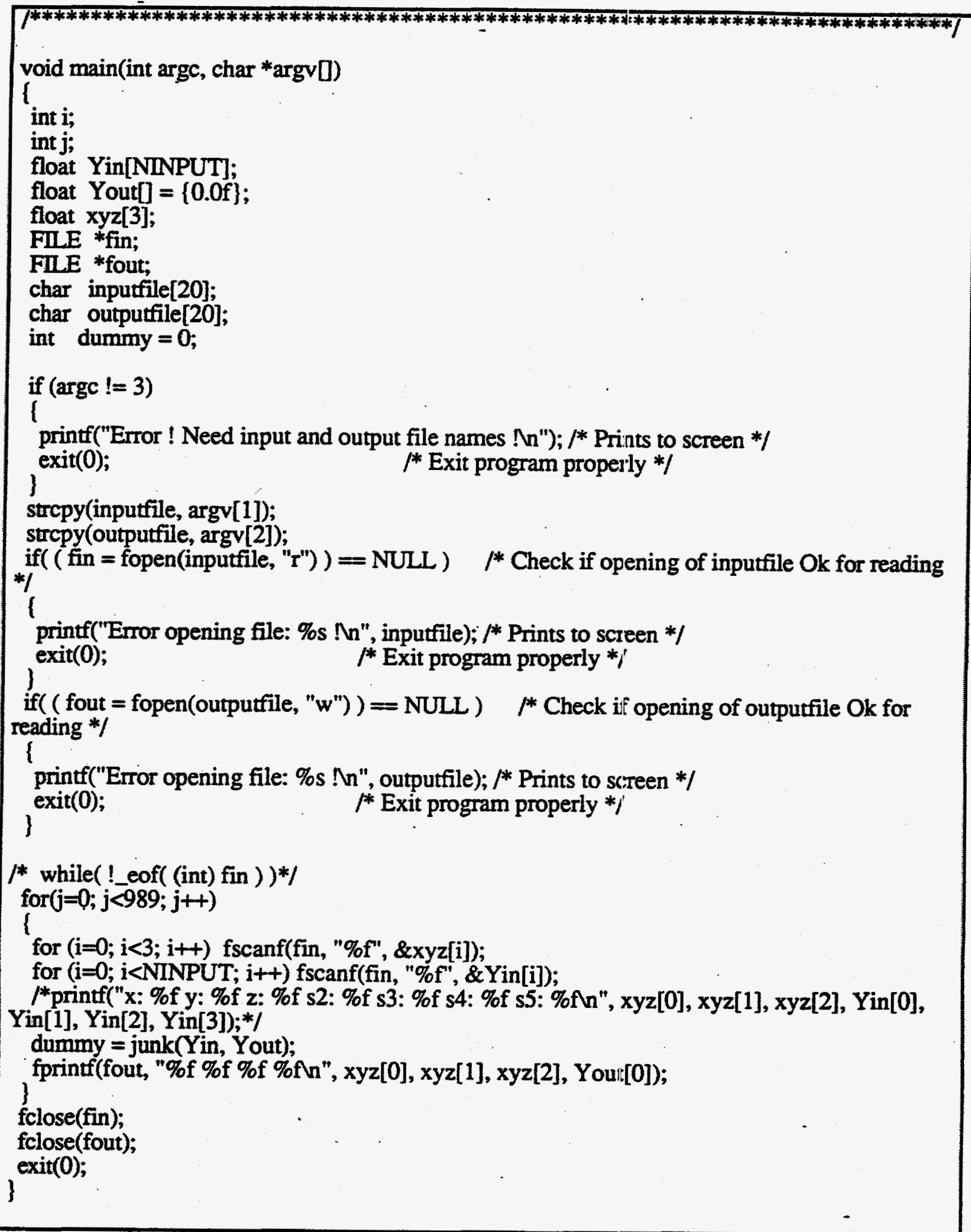




\section{Appendix D}

Descriptions of the RWMIIS and Qualifier Data Base Fields 
D-2 


\section{Appendix D}

\section{Descriptions of the RWMIIS and Qualifier Data Base Fields \\ D-1. SUMMARY}

This appendix contains a description of the Radioactive Waste Management Information System (RWMIS) data base fields. The descriptions and data base field names given below are those assigned by the CIDRA effort (Smith 1994).

\section{D-2. DESCRIPTIONS}

Tables D-1 through D-4 below give descriptions for the master, container, nuclide, and qualifier data bases field names. 
Table D-1. Descriptions of master data base fields.

\begin{tabular}{|c|c|}
\hline BAD_HST- & $\begin{array}{l}\text { Indicates if the data are from best available data (BAD) or historical data } \\
\text { (HST). Used as a bookkeeping field for the CIDRA project. Always } \\
\text { BAD for the Pit } 9 \text { entries }\end{array}$ \\
\hline HSAREA - & Area from which the waste originated \\
\hline HSWASTE_TP - & Waste type (solid or liquid) \\
\hline HSRADIO_AC - & Indicates whether or not the waste is radioactive \\
\hline HSONOFF_SI - & Indicates whether the waste generator was onsite or offsite \\
\hline HSREPORT_D - & Date the shipping form was completed \\
\hline HSREPORT_T - & Time the shipping form was completed \\
\hline HSWASTE_DE - & $\begin{array}{l}\text { Waste description (actually the radiation reading in millirem per hour } \\
\text { taken at contact and at } 1 \mathrm{~m} \text { ) }\end{array}$ \\
\hline HSGROSS_VO - & Gross volume of the waste in cubic meters \\
\hline HSGROSS_WG - & Gross weight of the waste in grams \\
\hline HSGROSS_AC - & Gross radioactivity of the waste in curies \\
\hline HDES_LOC - & General destination location (Pit 9 in this case) \\
\hline DOWNLOAD - & $\begin{array}{l}\text { Indicates which download of RWMIS. Used as bookkeeping field for } \\
\text { the CIDRA project. }\end{array}$ \\
\hline VERIFIED - & $\begin{array}{l}\text { Indicates if the record was verified against the shipping manifest. Used } \\
\text { as a bookkeeping field for the CIDRA project. }\end{array}$ \\
\hline TRU - & $\begin{array}{l}\text { Indicates if the shipment contains transuranic waste. Used as a } \\
\text { bookkeeping field for the CIDRA project. }\end{array}$ \\
\hline
\end{tabular}


Table D-2. Descriptions of container data base fields.

\begin{tabular}{|c|c|}
\hline BAD_HST- & $\begin{array}{l}\text { Indicates if the data are from best available data (BAD) or historical data } \\
\text { (HST). Used as a bookkeeping field for the CIDRA project. Always } \\
\text { BAD for the Pit } 9 \text { entries }\end{array}$ \\
\hline HSAREA - & Area from which the waste originated \\
\hline HSWASTE_TP - & Waste type (solid or liquid) \\
\hline HSRADIO_AC - & Indicates whether or not the waste is radioactive \\
\hline HSONOFF_SI - & Indicates whether the waste generator was onsite or offsite \\
\hline HSREPORT_D - & Date the shipping form was completed \\
\hline HSREPORT_T - & Time the shipping form was completed \\
\hline HSWASTE_DE - & $\begin{array}{l}\text { Waste description (actually the radiation reading in millirem per hour } \\
\text { taken at contact and at } 1 \mathrm{~m} \text { ) }\end{array}$ \\
\hline HTYPE_CONT - & Container type (box, barrel, ...) \\
\hline HNO_CONT1 & Number of waste containers in the shipment \\
\hline HCONT_VOL1 - & Volume of each waste container \\
\hline HVOL_UNIT1 - & Unit of volume for each waste container type ( $F-\mathrm{ft}^{3}, \mathrm{~K}$-gallons) \\
\hline HSOLID_DS1 & Content code providing a generic description of the waste contents \\
\hline HDES_LOC1 - & Specific disposal location of the waste \\
\hline HDES_DAT1 - & Disposal date \\
\hline HDES_LOC - & General destination location (Pit 9 in this case) \\
\hline DOWNLOAD - & $\begin{array}{l}\text { Indicates which download of RWMIS. Used as a bookkeeping field for } \\
\text { the CIDRA project. }\end{array}$ \\
\hline VERIFIED - & $\begin{array}{l}\text { Indicates if the record was verified against the shipping manifest. Used } \\
\text { as a bookkeeping field for the CIDRA project. }\end{array}$ \\
\hline TRU - & $\begin{array}{l}\text { Indicates if the shipment contains transuranic waste. Used as a } \\
\text { bookkeeping field for the CIDRA project. }\end{array}$ \\
\hline
\end{tabular}


Table D-3. Descriptions of nuclide data base fields.

\begin{tabular}{|c|c|}
\hline BAD_HST- & $\begin{array}{l}\text { Indicates if the data are from best available data (BAD) or historical data } \\
\text { (HST). Used as a bookkeeping field for the CIDRA project. Always } \\
\text { BAD for the Pit } 9 \text { entries }\end{array}$ \\
\hline HSAREA - & Area from which the waste originated \\
\hline HSWASTE_TP - & Waste type (solid or liquid) \\
\hline HSRADIO_AC - & Indicates whether or not the waste is radioactive \\
\hline HSONOFF_SI - & Indicates whether the waste generator was onsite or offsite \\
\hline HSREPORT_D - & Date the shipping form was completed \\
\hline HSREPORT_T - & Time the shipping form was completed \\
\hline HSWASTE_DE - & $\begin{array}{l}\text { Waste description (actually the radiation reading in millirem per hour } \\
\text { taken at contact and at } 1 \mathrm{~m} \text { ) }\end{array}$ \\
\hline HNUC_ID2 - & Isotopic nuclide designation \\
\hline HNUC_CON2 - & Gram quantity of each nuclide \\
\hline HNUC_CUR2 - & Curied quantity of each nuclide \\
\hline HNUC_NO2 - & N/A for Pit 9 \\
\hline HNUC_LES2 - & N/A for Pit 9 \\
\hline DOWNLOAD - & $\begin{array}{l}\text { Indicates which download of RWMIS. Used as bookkeeping field for } \\
\text { the CIDRA project. }\end{array}$ \\
\hline HDES_LOC - & General destination location (Pit 9 in this case) \\
\hline VERIFIED - & $\begin{array}{l}\text { Indicates if the shipment contains transuranic waste. Used as a } \\
\text { bookkeeping field for the CIDRA project. }\end{array}$ \\
\hline TRU - & $\begin{array}{l}\text { Indicates if the shipment contains transuranic waste. Used as a } \\
\text { bookkeeping field for the CIDRA project. }\end{array}$ \\
\hline
\end{tabular}


Table D-4. Descriptions of qualifier flag data base fields.

\begin{tabular}{|c|c|}
\hline SDA_LOC - & Location of the area in the SDA \\
\hline HSAREA - & Area from which the waste originated \\
\hline HSWASTE_TP - & Waste type (solid or liquid) \\
\hline HSRADIO_AC - & Indicates whether or not the waste is radioactive \\
\hline HSONOFF_SI - & Indicates whether the waste generator was onsite or offsite \\
\hline HSREPORT_D - & Date the shipping form was completed \\
\hline HSREPORT_T - & Time the shipping form was completed \\
\hline HSWASTE_DE - & $\begin{array}{l}\text { Waste description (actually the radiation reading in millirem } \\
\text { per hour taken at contact and at } 1 \mathrm{~m} \text { ) }\end{array}$ \\
\hline SHIP_MISS - & Indicates if the shipping manifest is missing \\
\hline ILLEGIBLE - & $\begin{array}{l}\text { Holds the entry codes for fields that are illegible on the } \\
\text { shipment record }\end{array}$ \\
\hline SHIP_BLANK - & $\begin{array}{l}\text { Holds the entry codes for fields that are blank on the } \\
\text { shipment record and nozero in RWMIS }\end{array}$ \\
\hline ZERO_BLANK - & $\begin{array}{l}\text { Holds the entry codes for fields that are blank on the } \\
\text { shipment record and zero in RWMIS }\end{array}$ \\
\hline CONTCODE(1 through 10$)$-. & $\begin{array}{l}\text { Holds a content code number and the information that is } \\
\text { different between the content code in REMIS and the } \\
\text { information on the shipment record. }\end{array}$ \\
\hline MISCELL - & Holds general comments \\
\hline UPDATE - & Indicates if the associated entry in RWMIS was updated \\
\hline KEY_FIELD - & $\begin{array}{l}\text { Indicates if the change to be made is in one of the RWMIS } \\
\text { key fields }\end{array}$ \\
\hline OLD_KEY - & Holds the old key prior to the update \\
\hline TRU - & $\begin{array}{l}\text { Indicates if the shipment contains transuranic waste. Used as } \\
\text { a bookkeeping field for the CIDRA project. }\end{array}$ \\
\hline
\end{tabular}




\section{D-3. REFERENCES}

Smith, T. H. et al., 1994, A Comprehensive Inventory of Radiological and Nonradiological Contaminants in Waste Buried in the Subsurface Disposal Area of the INEL RWMC During the Years 1952-1983, EGG-WM-10903, EG\&G Idaho, Inc., June. 


\section{Appendix E}

Printouts of RWMIS and Qualifier Data Bases 



\section{Appendix E}

\section{Printouts of RWMIs and Qualifier Data Bases}

\section{E-1. SUMMARY}

This appendix provides listings of the master, container, nuclide, and qualifier data bases for the Radioactive Waste Management Information System (RWMIS) l?it 9 entries. These data bases are products of the CIDRA effort (Smith 1994). 
Table E-1. Pit 9 RWMIS master file.

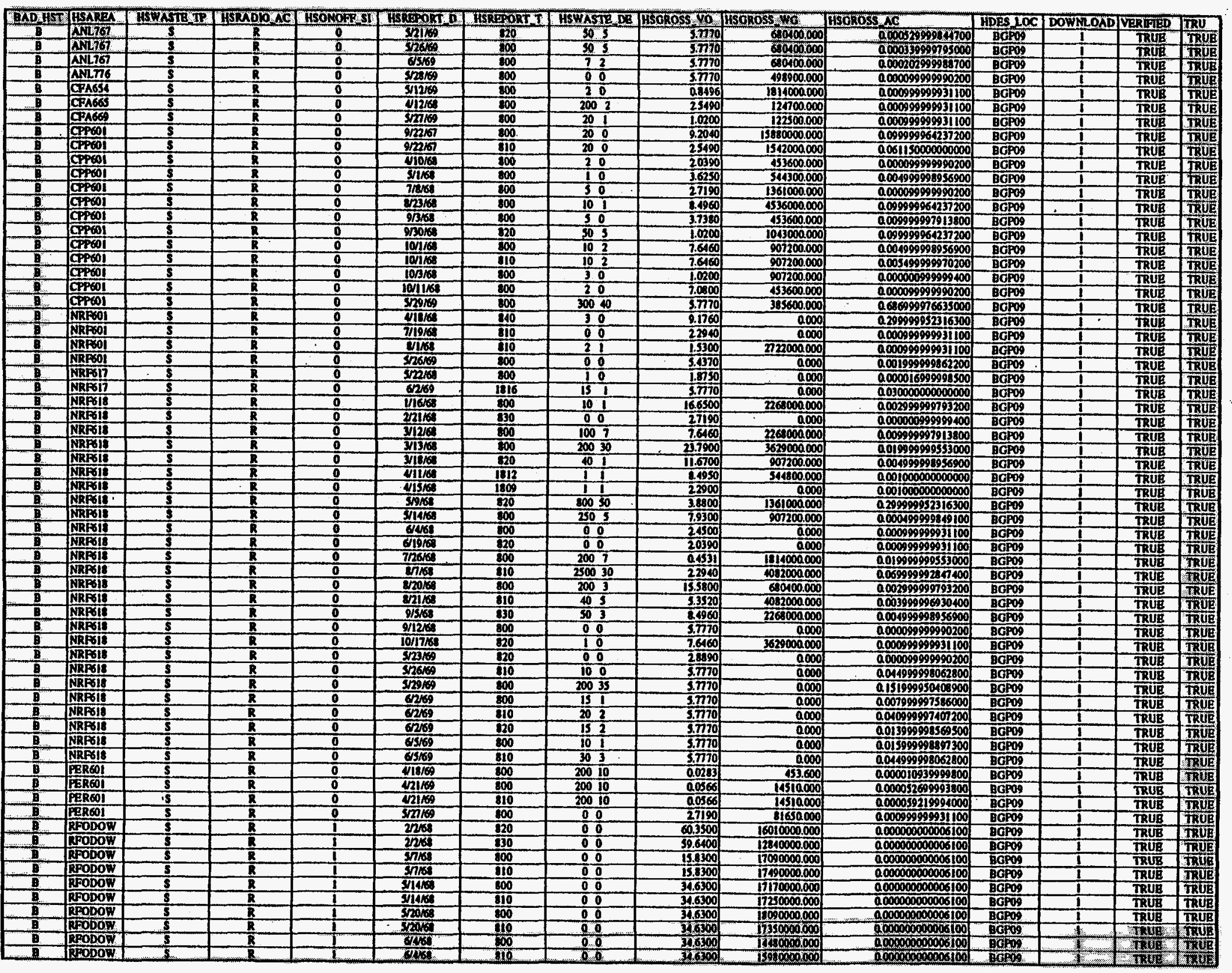


Table E-1. (continued).

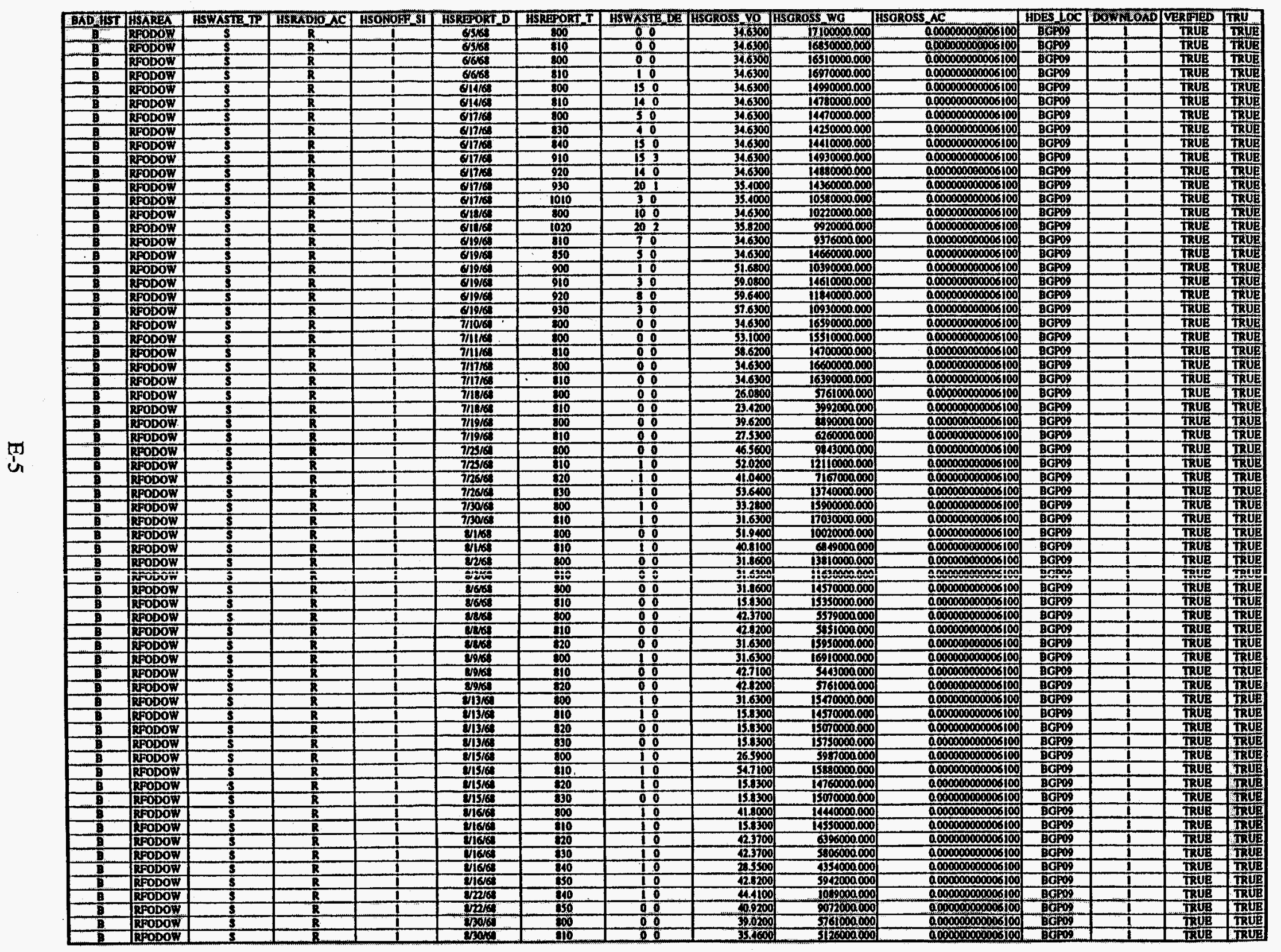




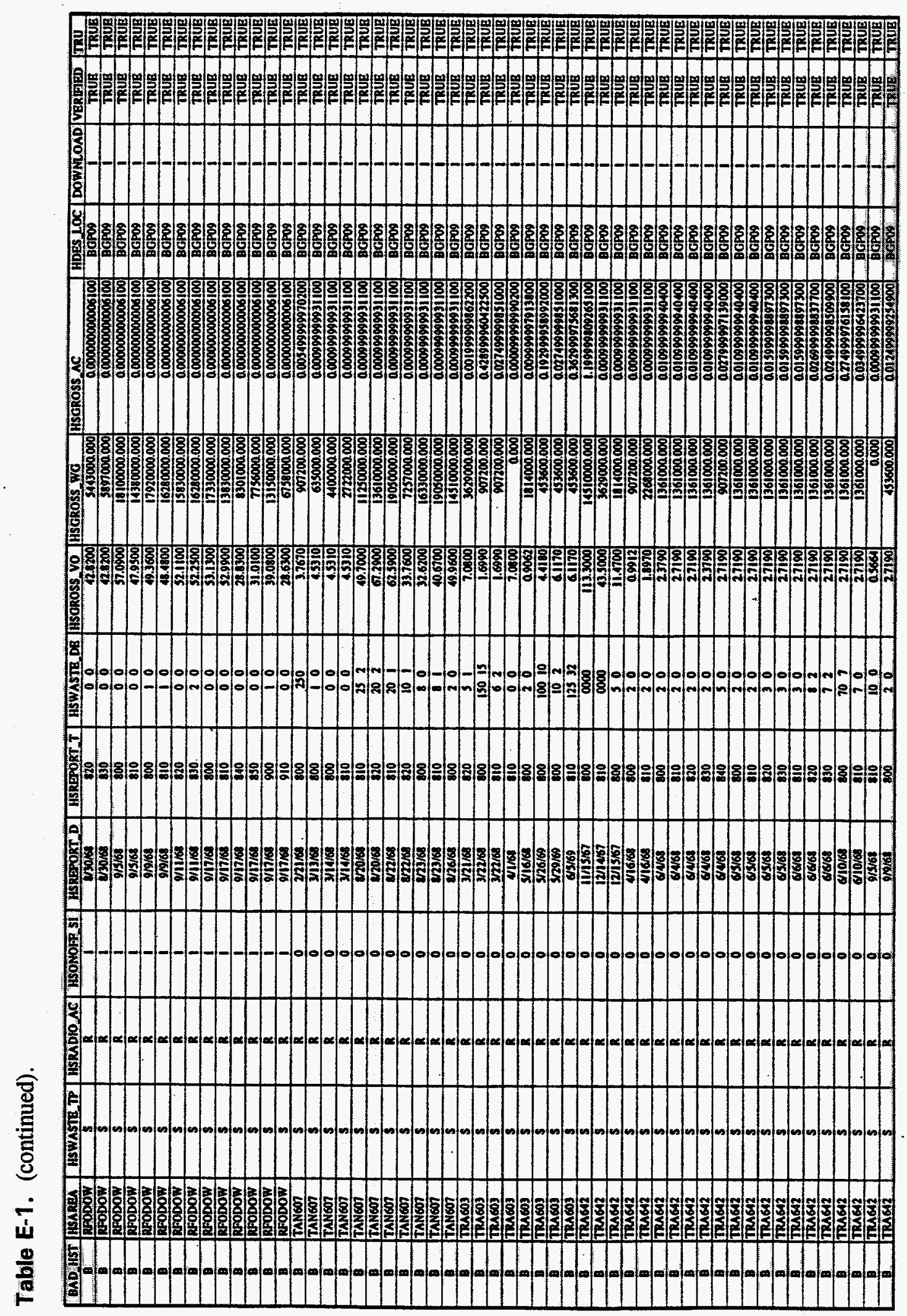

E-6 


눈

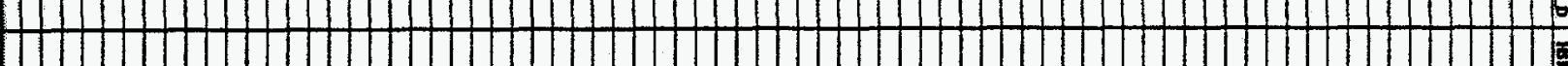

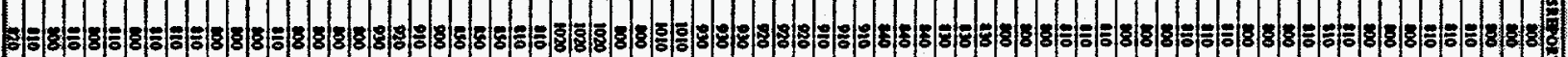
100

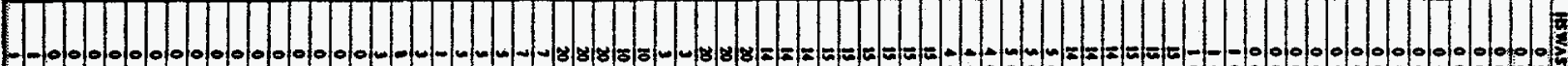

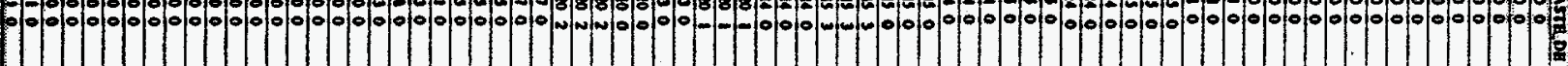
10 DD

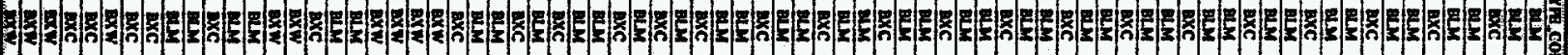
10020

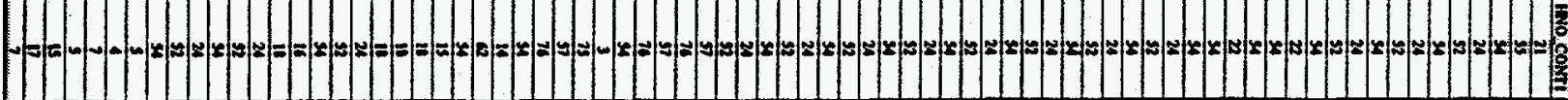

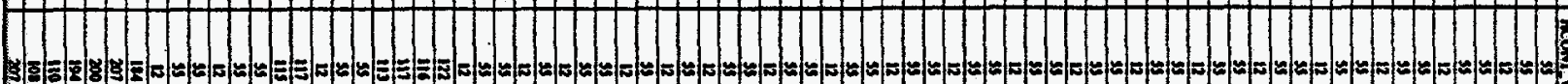

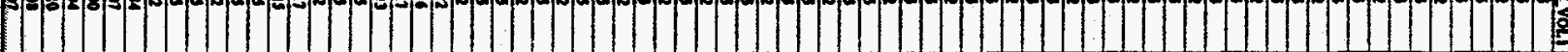

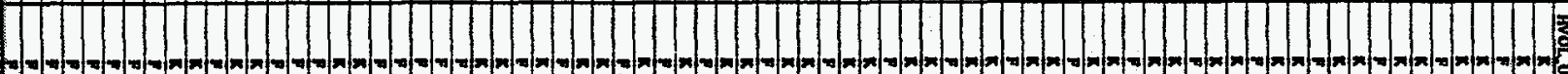

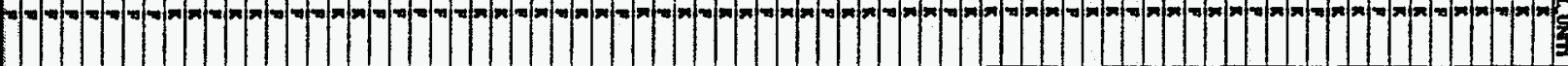
연

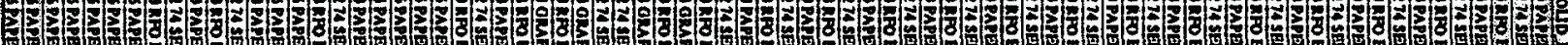
间.

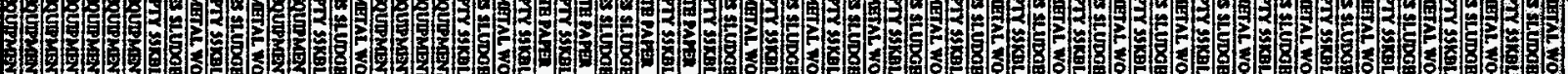

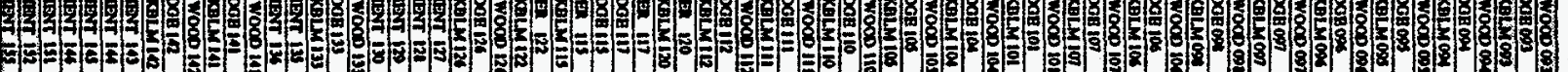
可

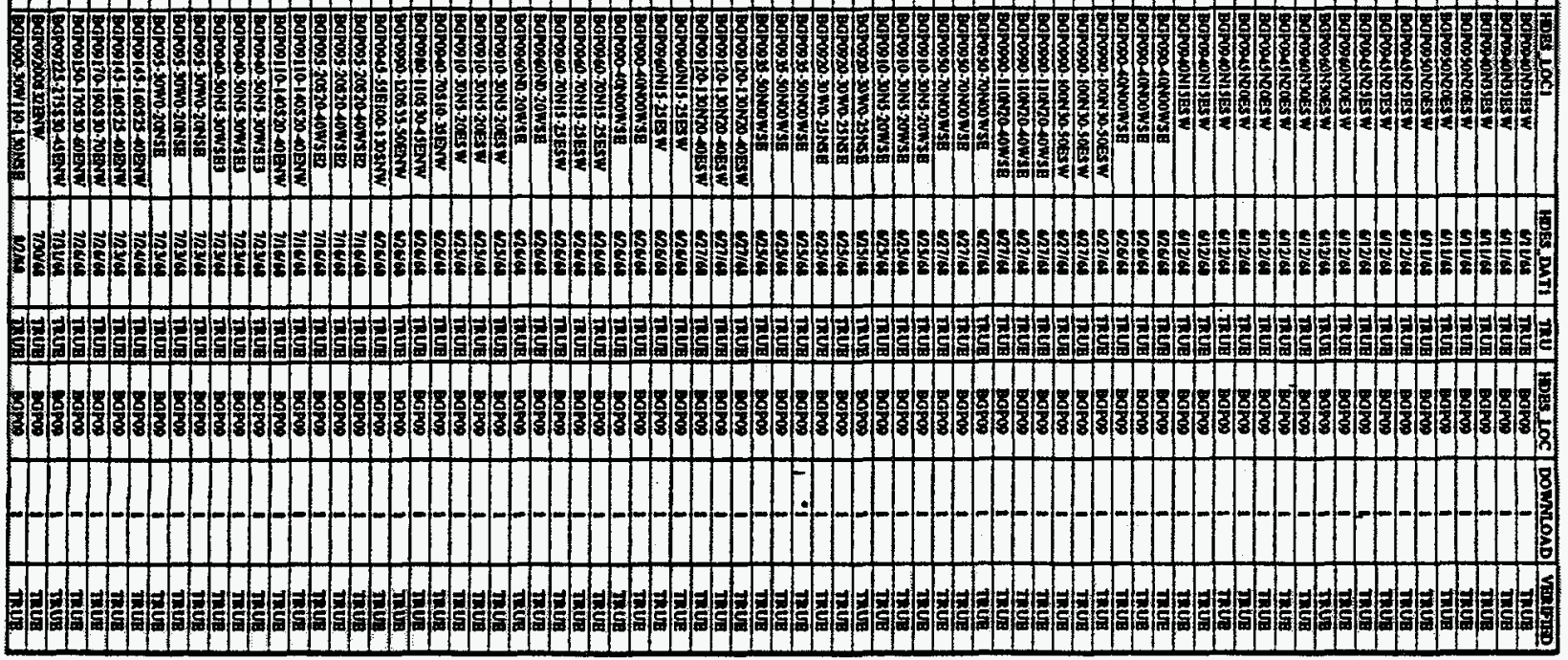


Table E-2. (continued).

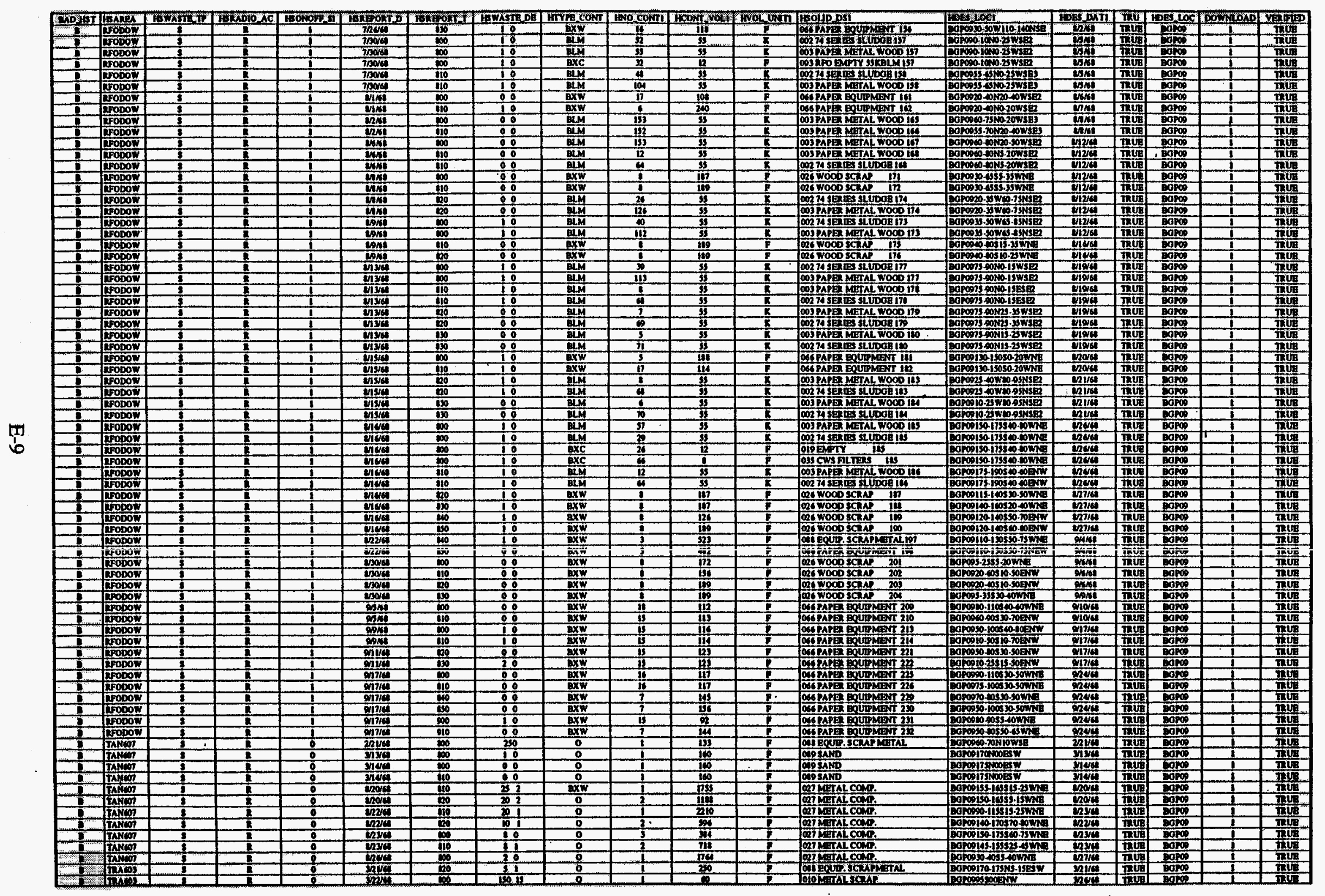


Table E-2. (continued).

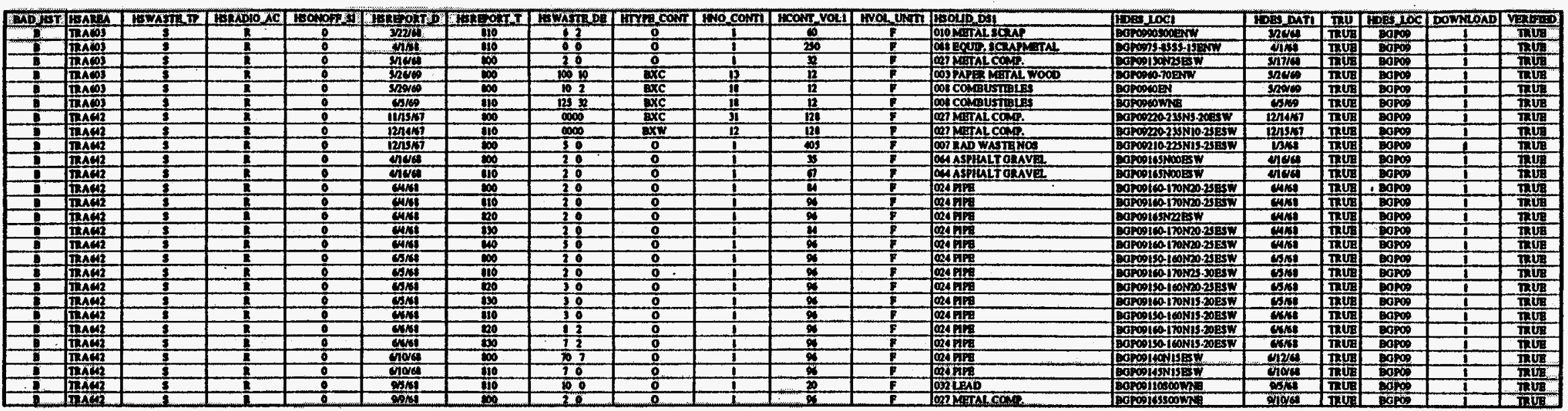




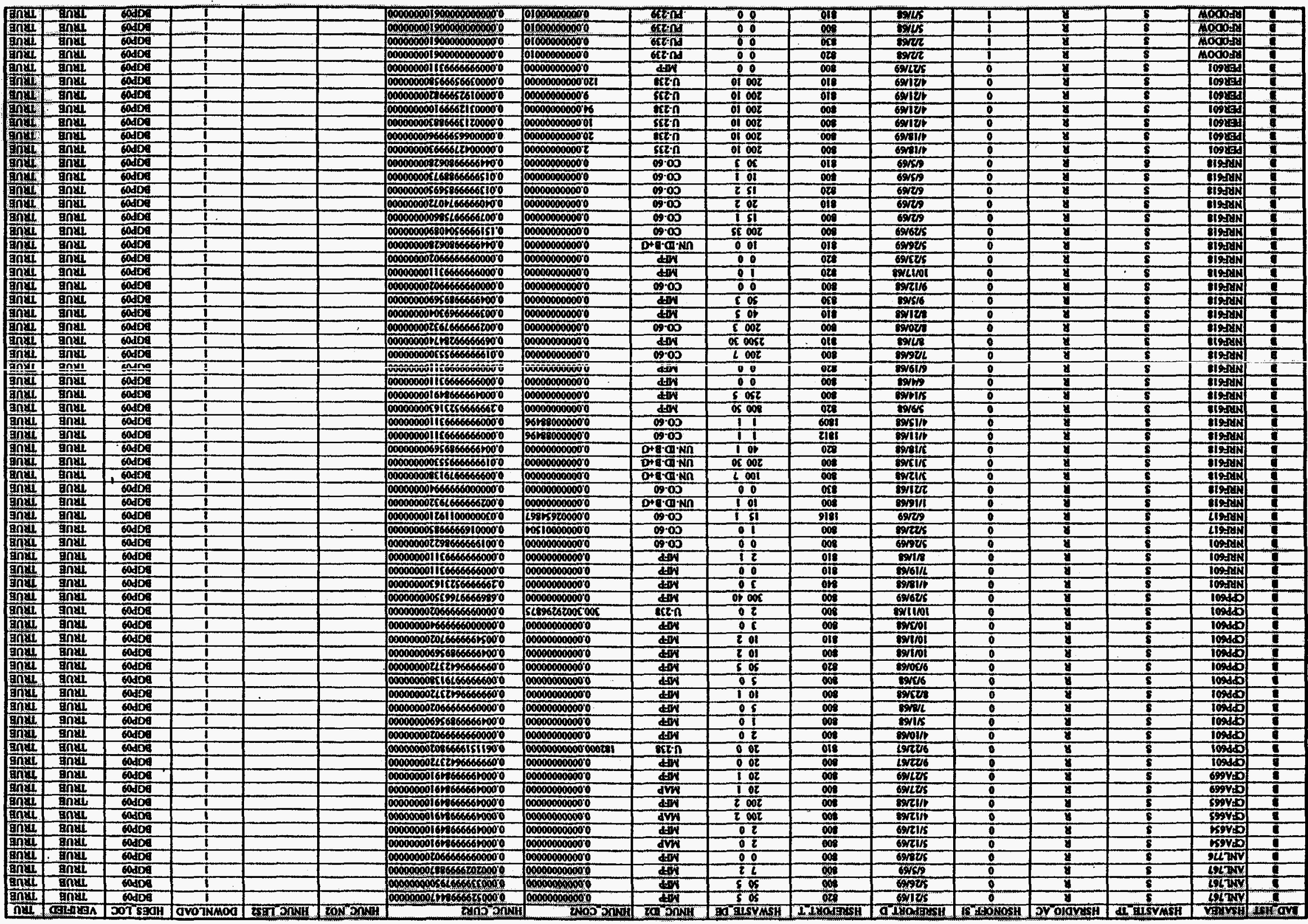


Table E-3. (continued).

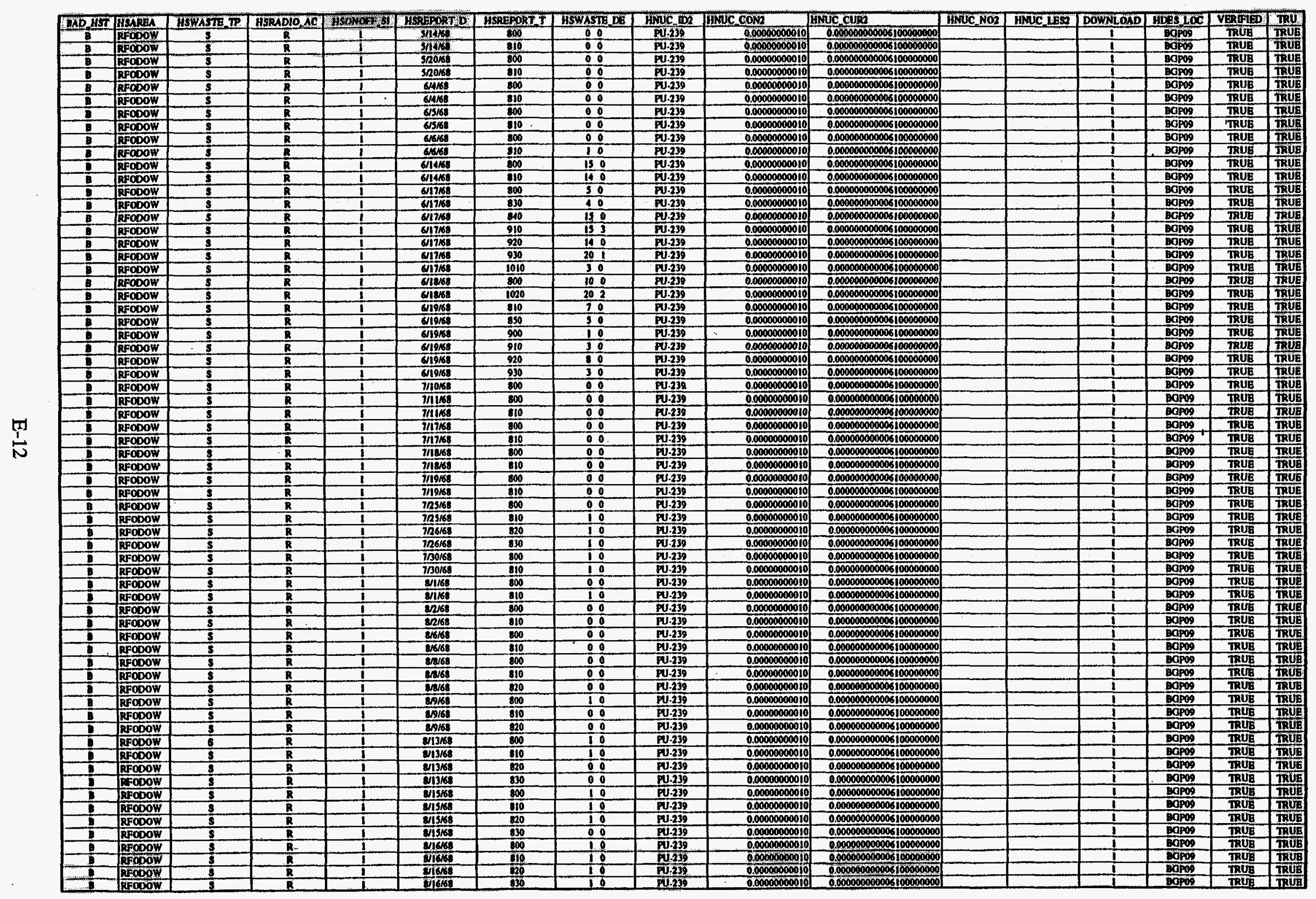


Table E-3. (continued).

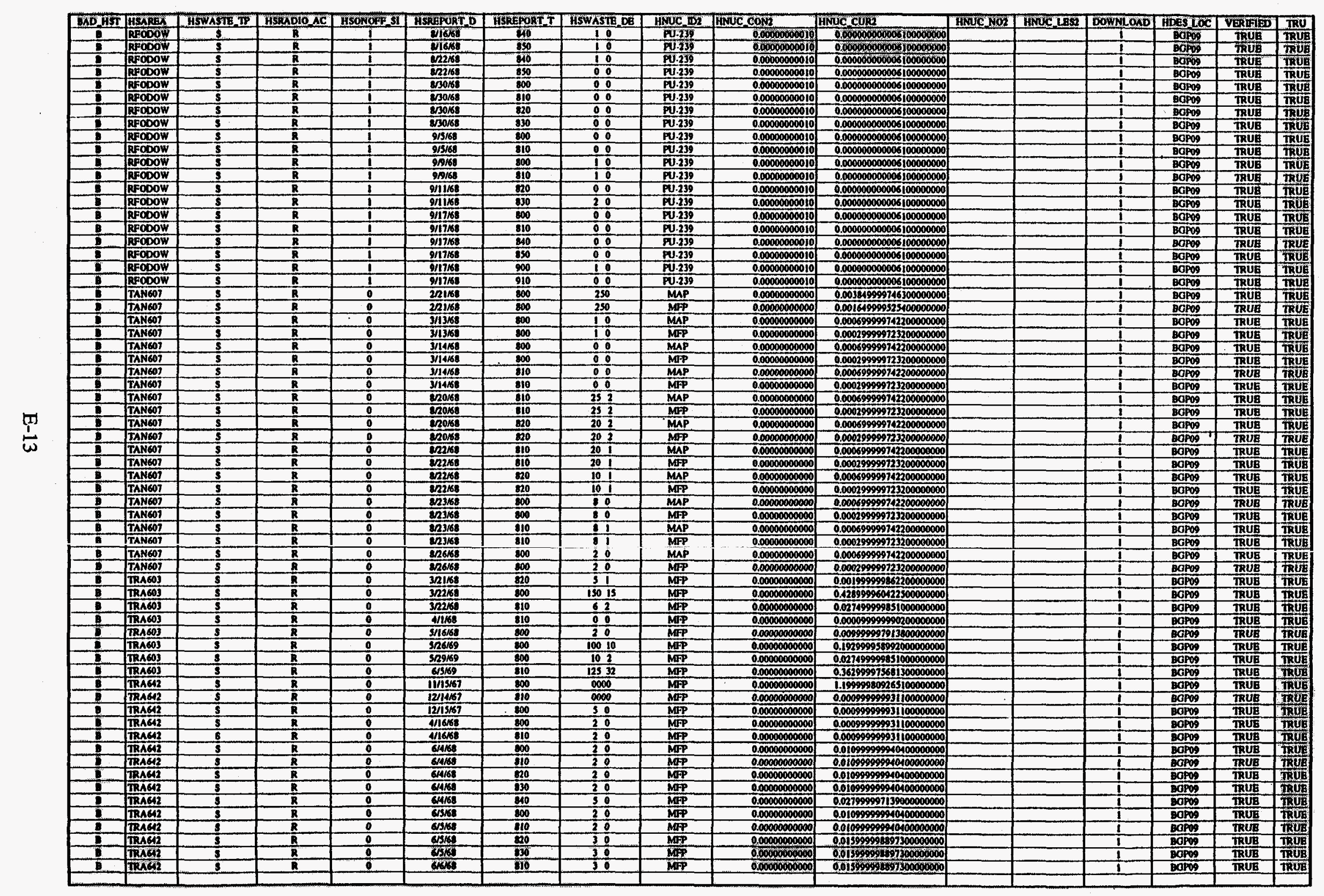


Table E-3. (continued).

\begin{tabular}{|c|c|c|c|c|c|c|c|c|c|c|c|c|c|c|c|c|}
\hline gnD.HS & HSAREA & HSWASTE IP & HISRADIOAAC & HSONOFESI & BSPRERORT D & HSPEPORTI & HSWASTE DE & HNuE_Di & IFNuC Coy & fiNuc_CUR & HNUE NO2 & HNOCDIS & DowiloAD & Hoss LOC & VERTEDE & तथ \\
\hline 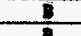 & TRAGZ & 5 & $-a$ & 0 & GKS & 20 & $\frac{2}{3}$ & $\mathrm{Mip}$ & 0.0000000000 & 0.0260000037700000000 & & & $\frac{1}{1}$ & 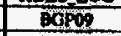 & TRÚ⿱一𫝀口) & TEUE \\
\hline 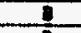 & magiz & 3 & B & 0 & 60165 & 830 & 72 & MaP & 0.00000000000 & 0.0249099060509000000000 & & & $i$ & BOPOP & TU1 & TRUE \\
\hline$D$ & pas? & 3 & B & 0 & Q10\%5 & 800 & 707 & MPP & 0.00000000000 & 0.2719999276189100000000 & & & i & Bopog & TRUE & TiNOE \\
\hline B & TRAG2 & $s$ & R & 0 & Q & 010 & 70 & MPP & 0.000000000000 & 0.034909996423700000000 & & & $i$ & BOPOO & TRUB & Tाus \\
\hline $\mathbf{e}$ & TRAGA2 & 5 & I & 0 & $9 / 563$ & 010 & 100 & MPP & 0.00000000000 & 0.00009999992111000000000 & & & i & BGPOS & TRUE & TRUE \\
\hline I & Exach & 3 & B. & 0 & 20063 & 80 & 20 & $\mathrm{MPB}$ & 0.00000000000 & 0.0126082825060 & & & & ECPOE & TRUE & TIUUE \\
\hline
\end{tabular}



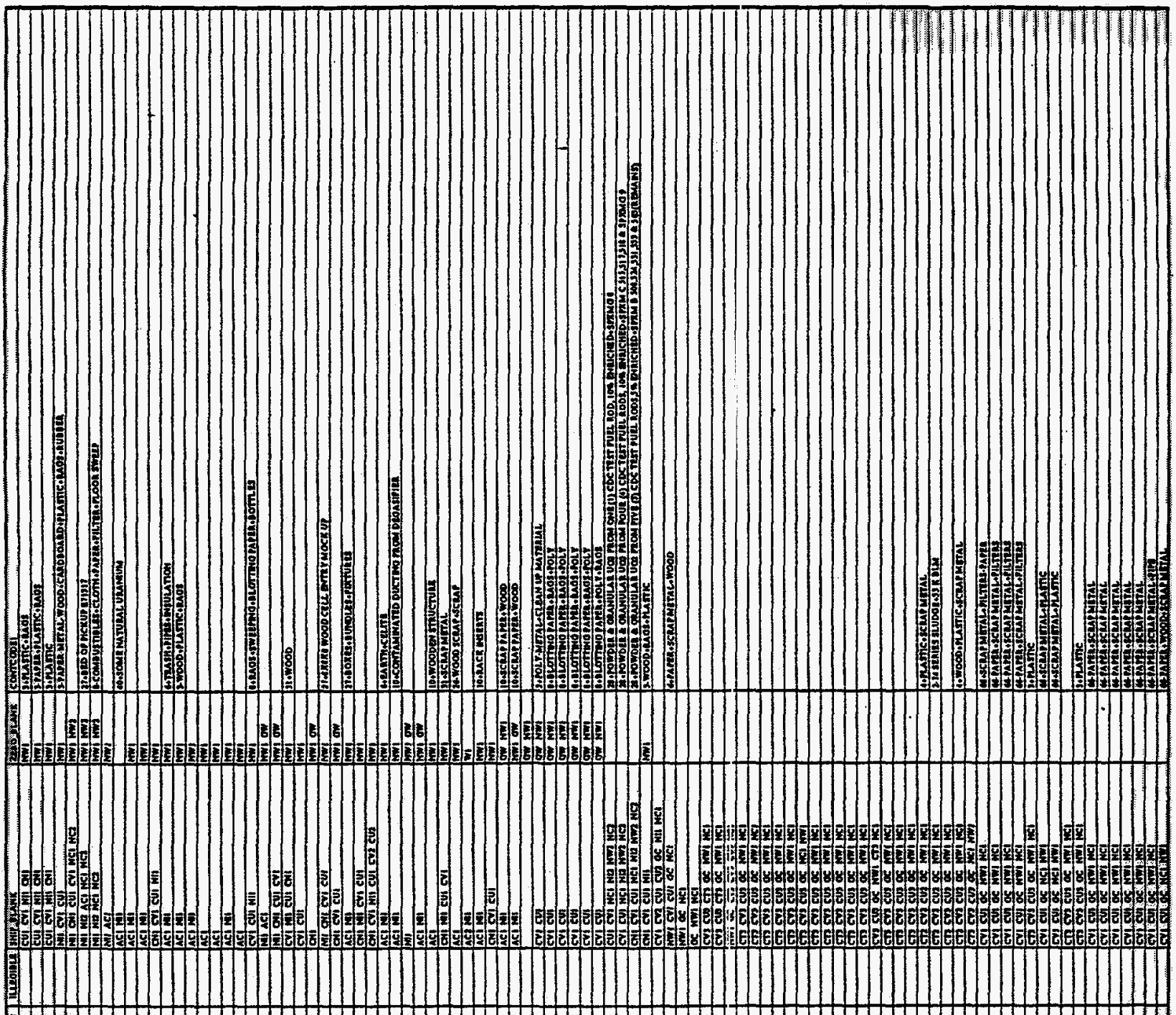

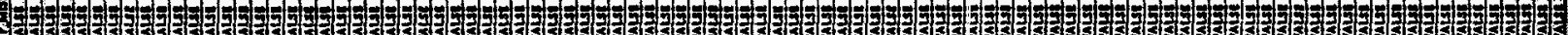

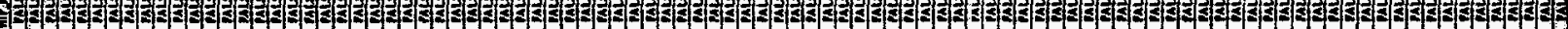

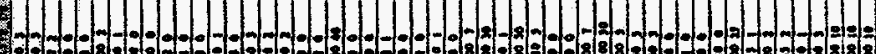

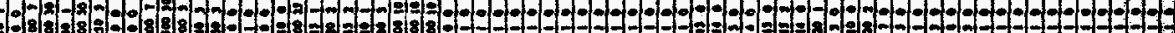
(1)

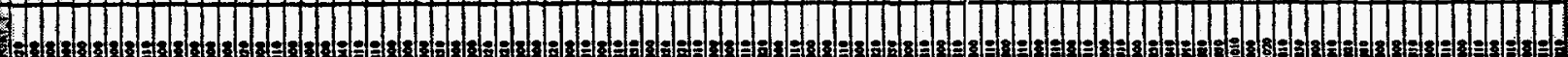
月1

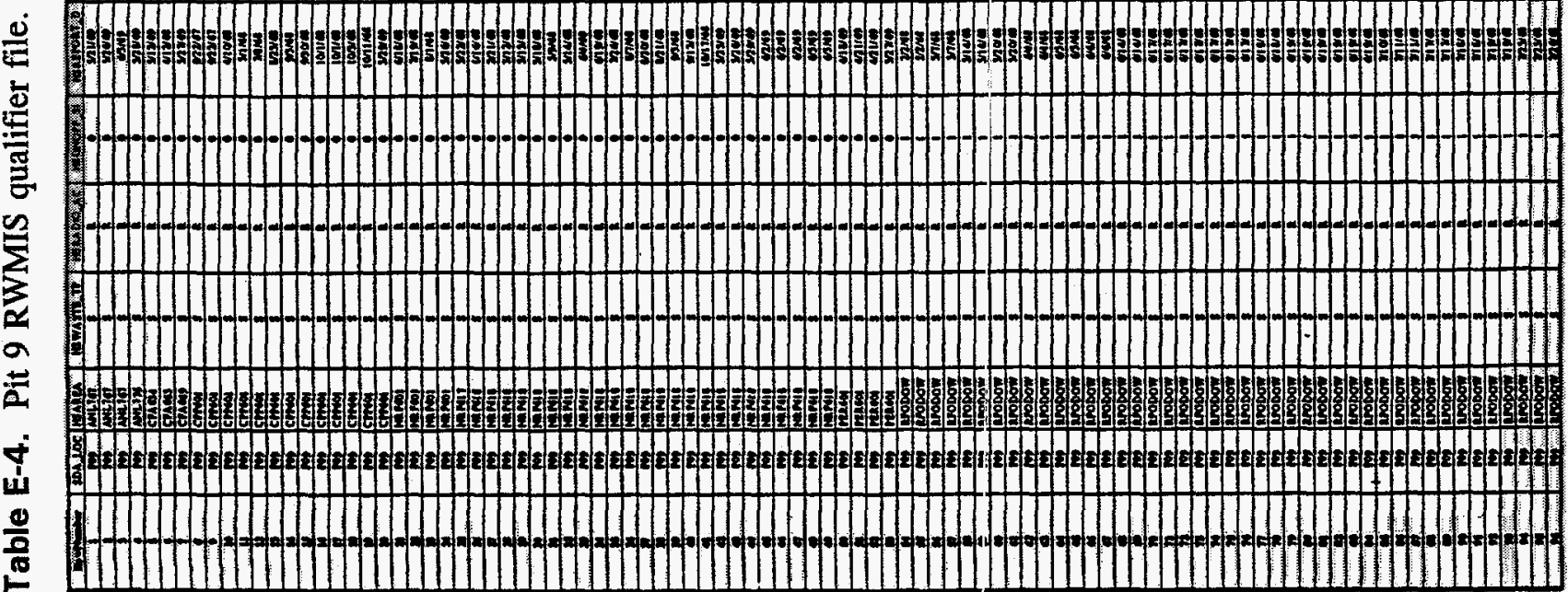


Table E-4. (continued).

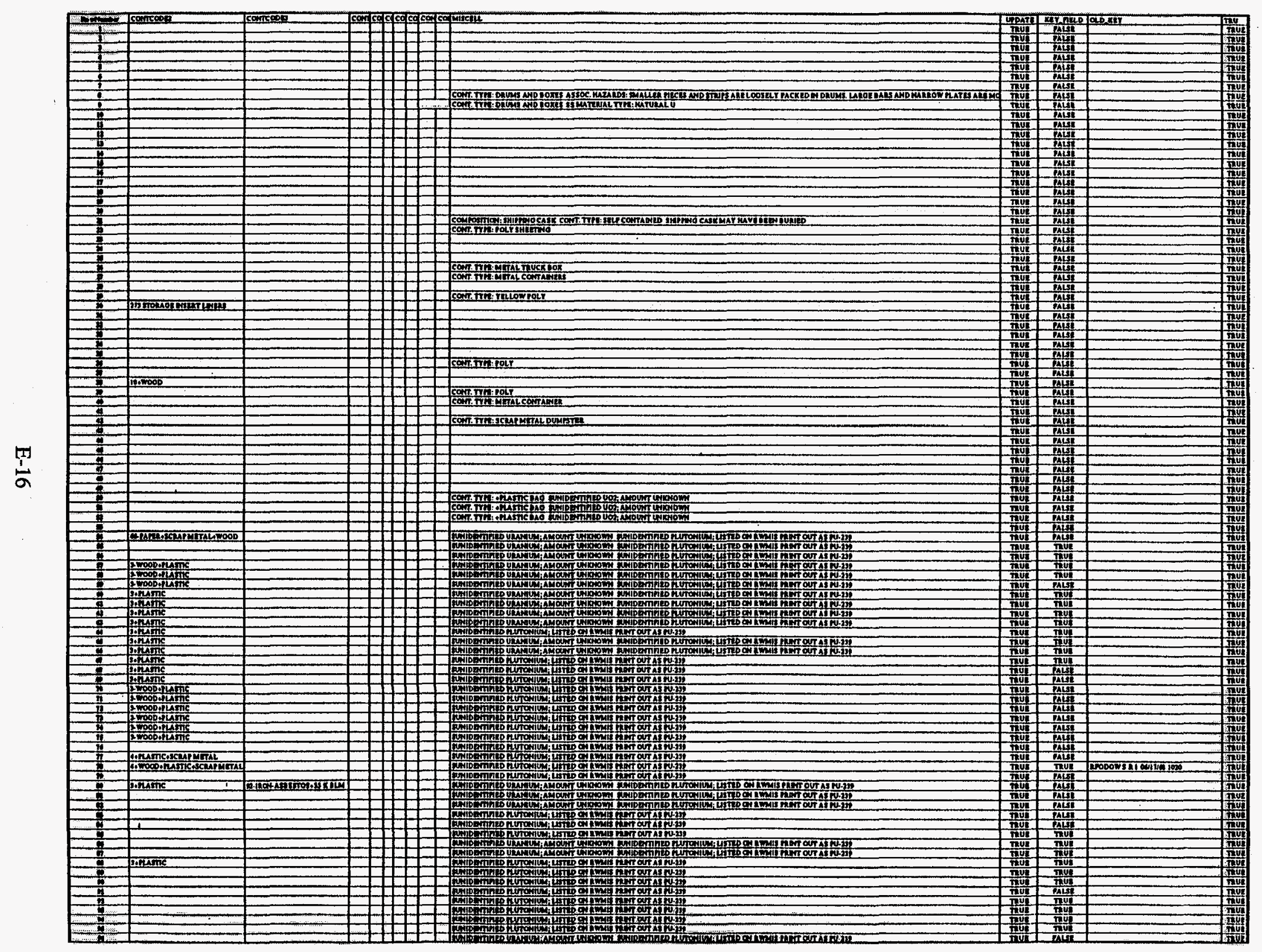




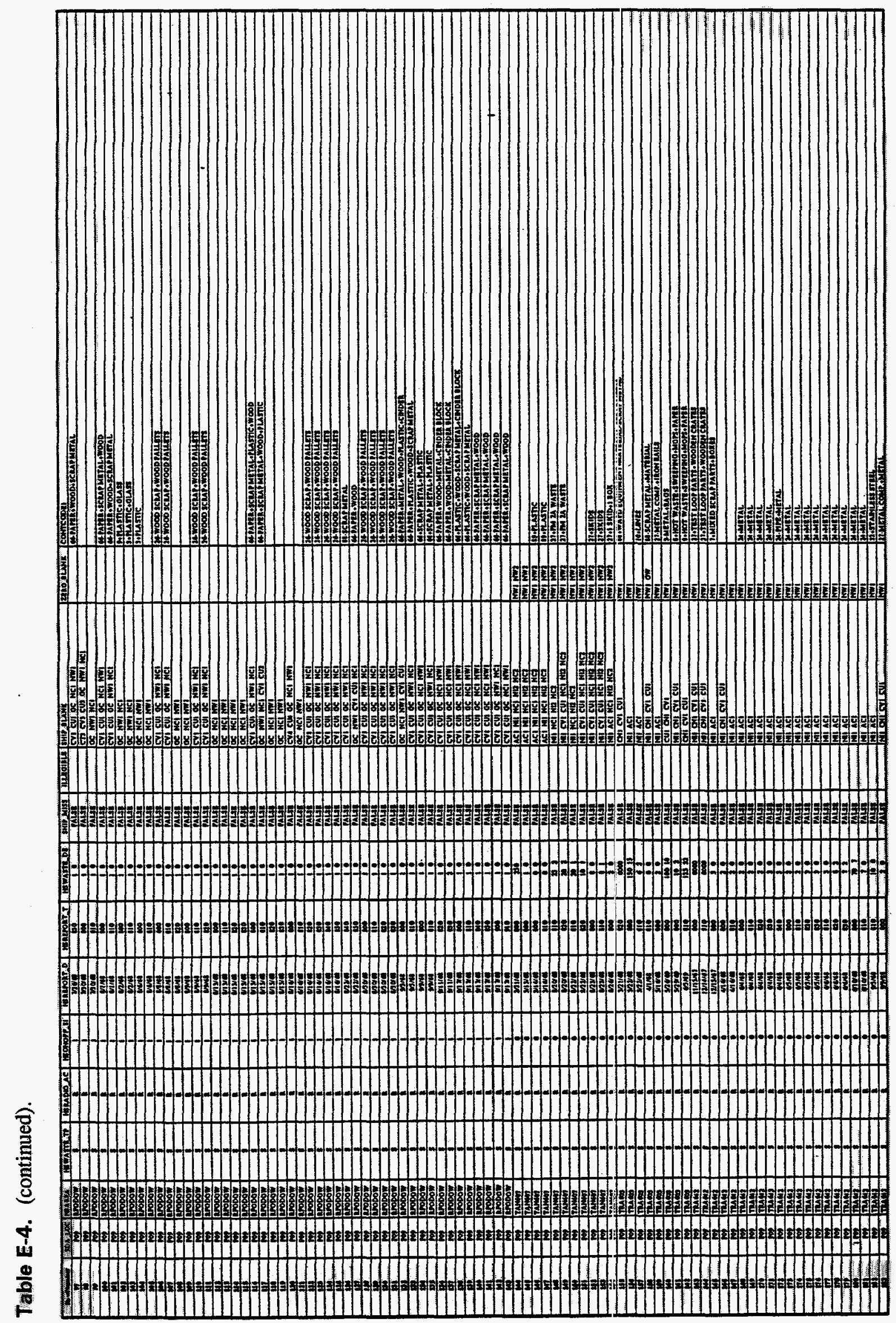

E-17 


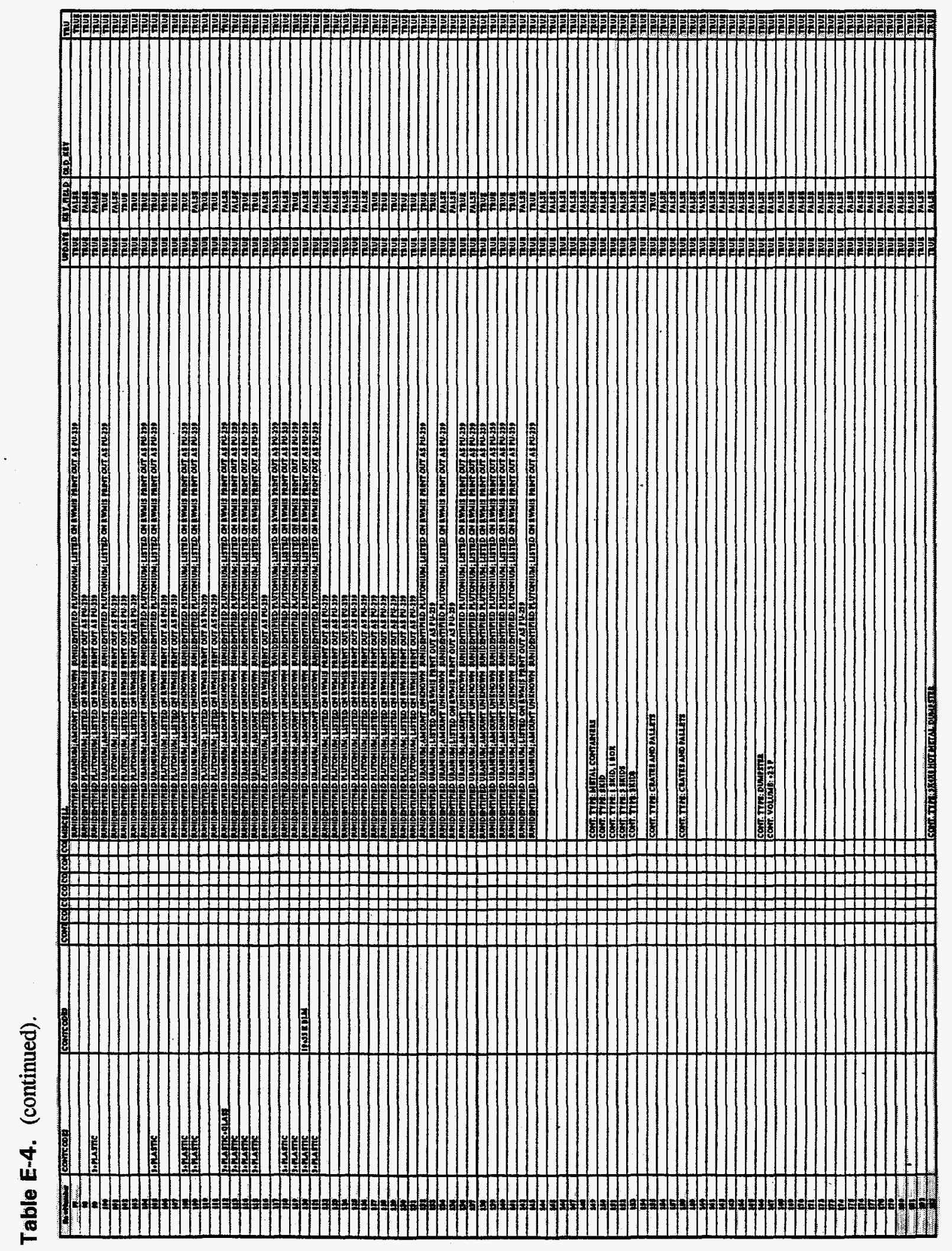

E-18 


\section{E-2. REFERENCES}

Smith, T. H. et al., 1994, A Comprehensive Inventory of Radiological and Nonradiological Contaminants in Waste Buried in the Subsurface Disposal Area of the INEL RWMC During the Years 1952-1983, EGG-WM-10903, EG\&G Idaho, Inc., June. 
Appendix F

Additions to WorldToolKit Source Code 
F-2 


\section{Appendix F}

\section{Additions to WorldToolKit Source Code}

\section{F-1. INTRODUCTION}

This appendix contains the source code additions to the WorldToolKit driver code required to allow access to the Aurora data base server.

\section{F-2. SOURCE CODE}

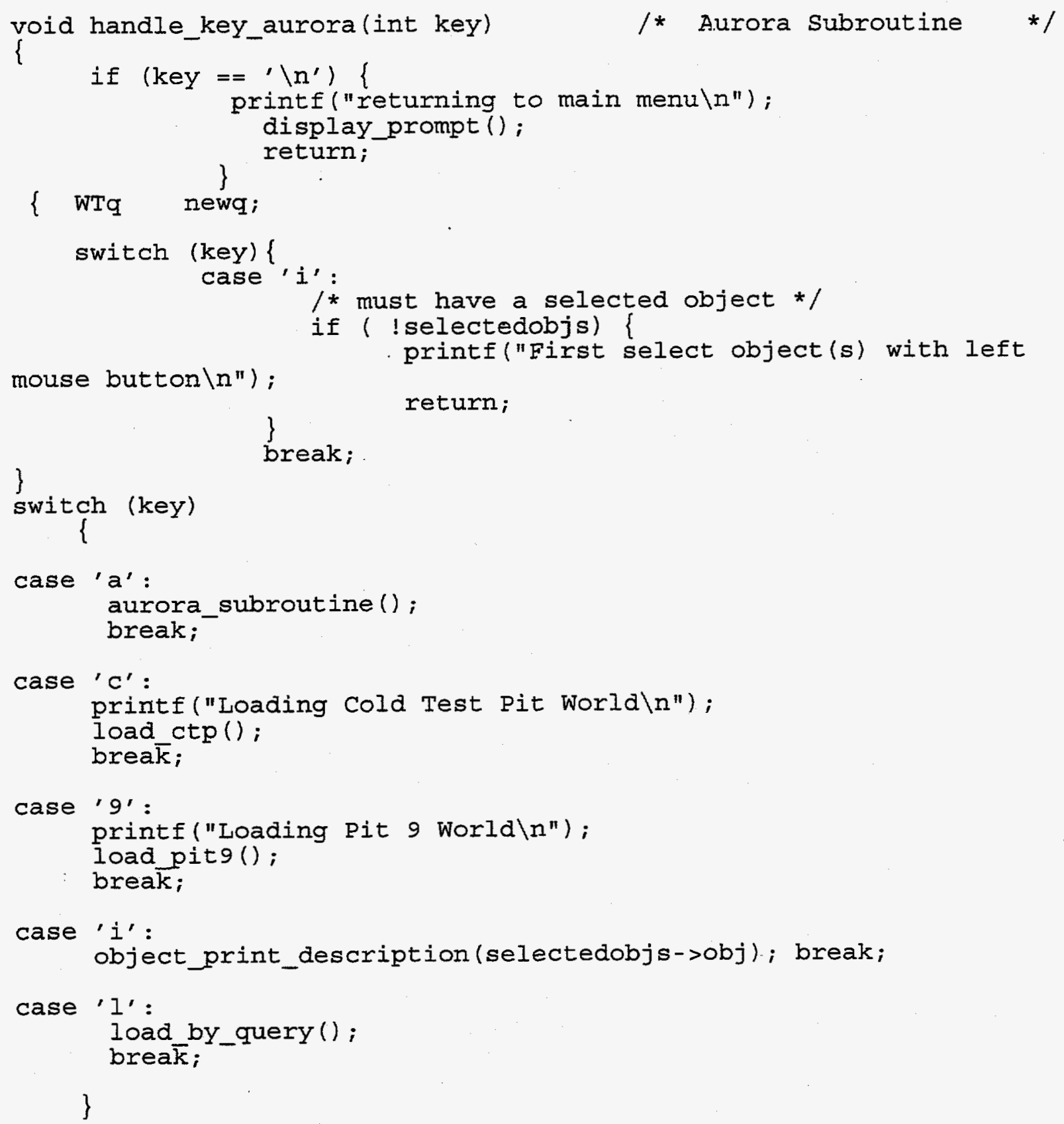




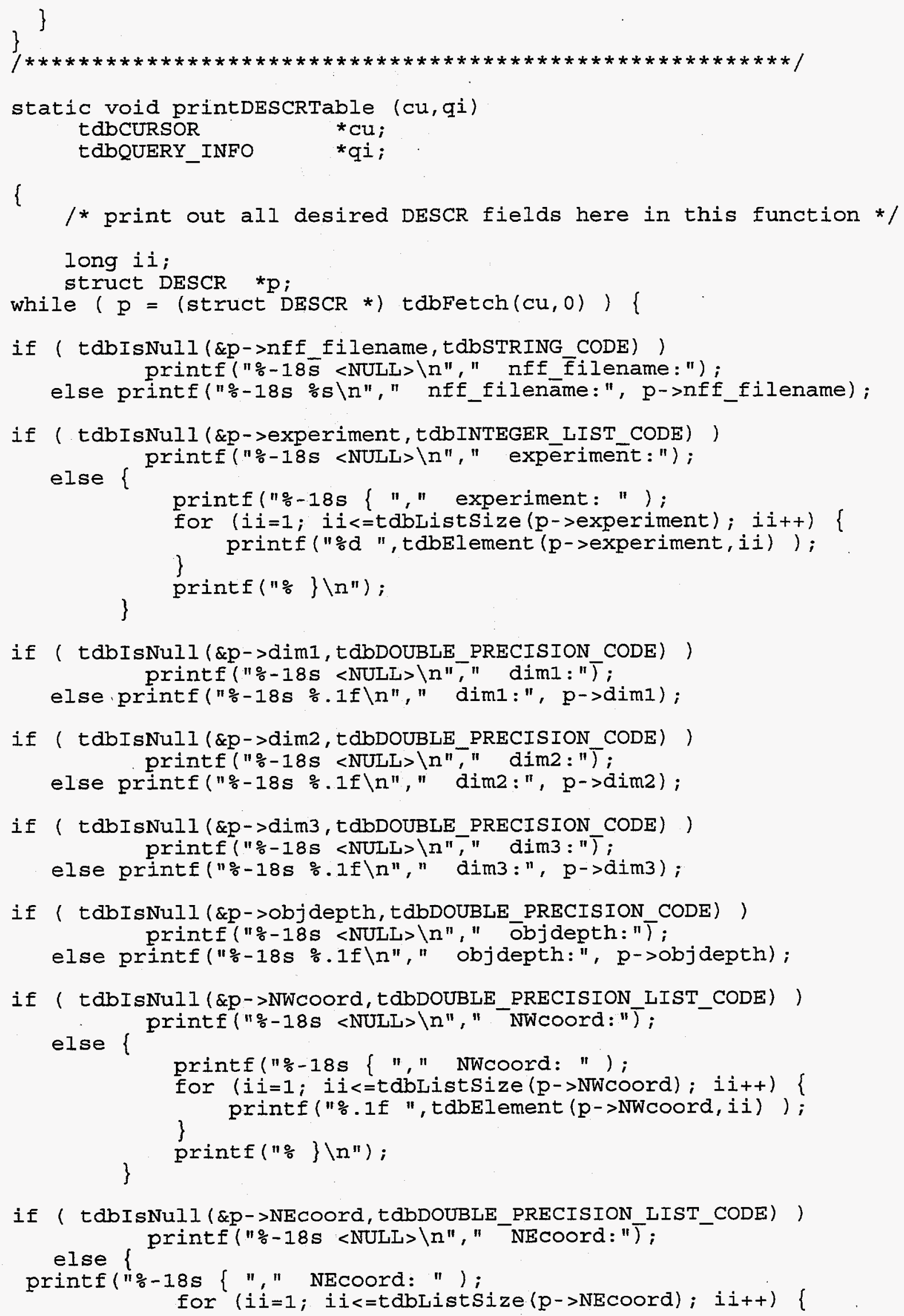

if ( tabIsNull ( $\&$ p->NEcOord, tdbDOUBLE_PRECISION_LIST_CODE) ) else printf $(" \%-18 \mathrm{~s}<$ NULL> $\backslash \mathrm{n} ", "$ NECoord: " $)$; 


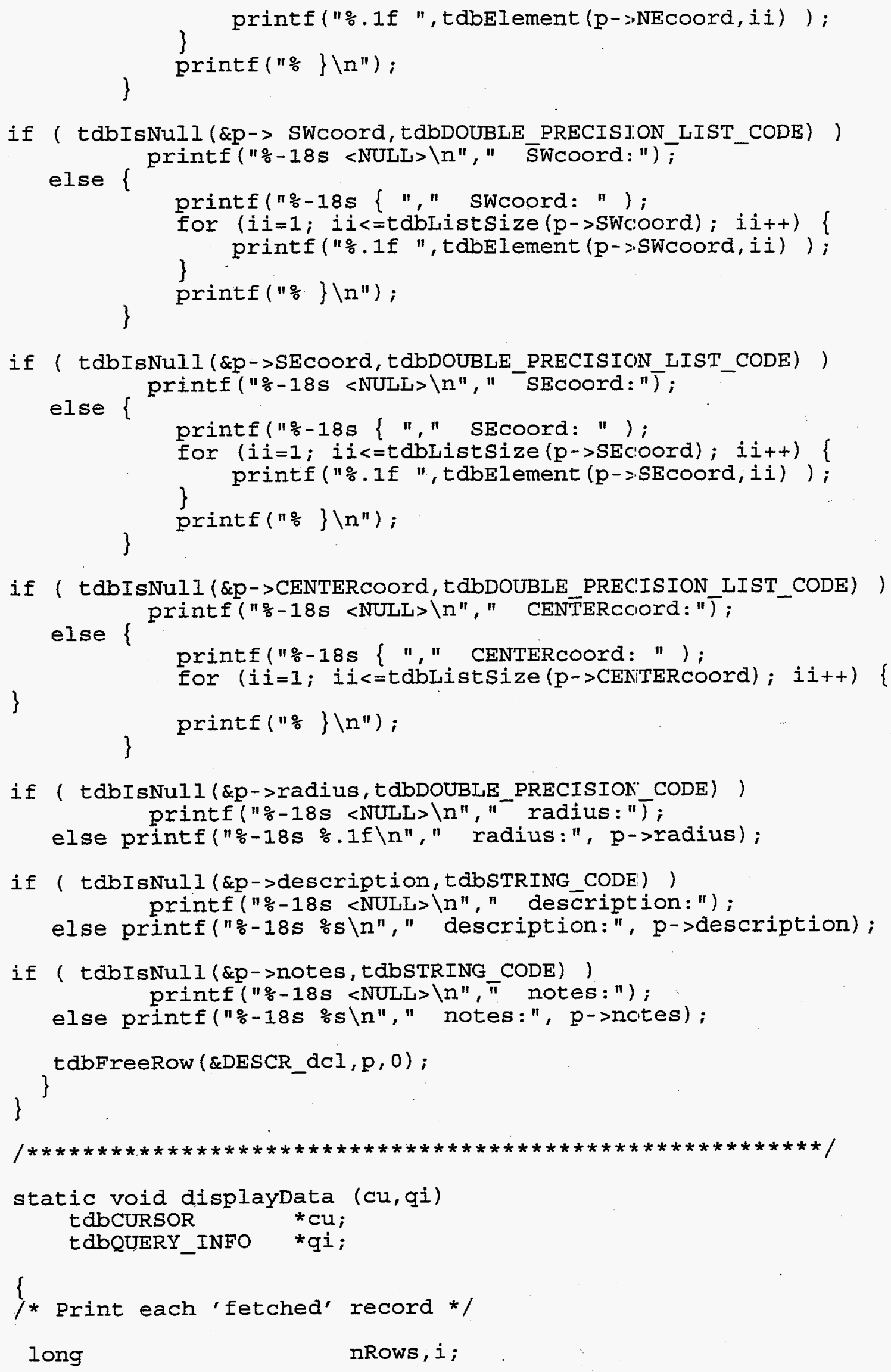




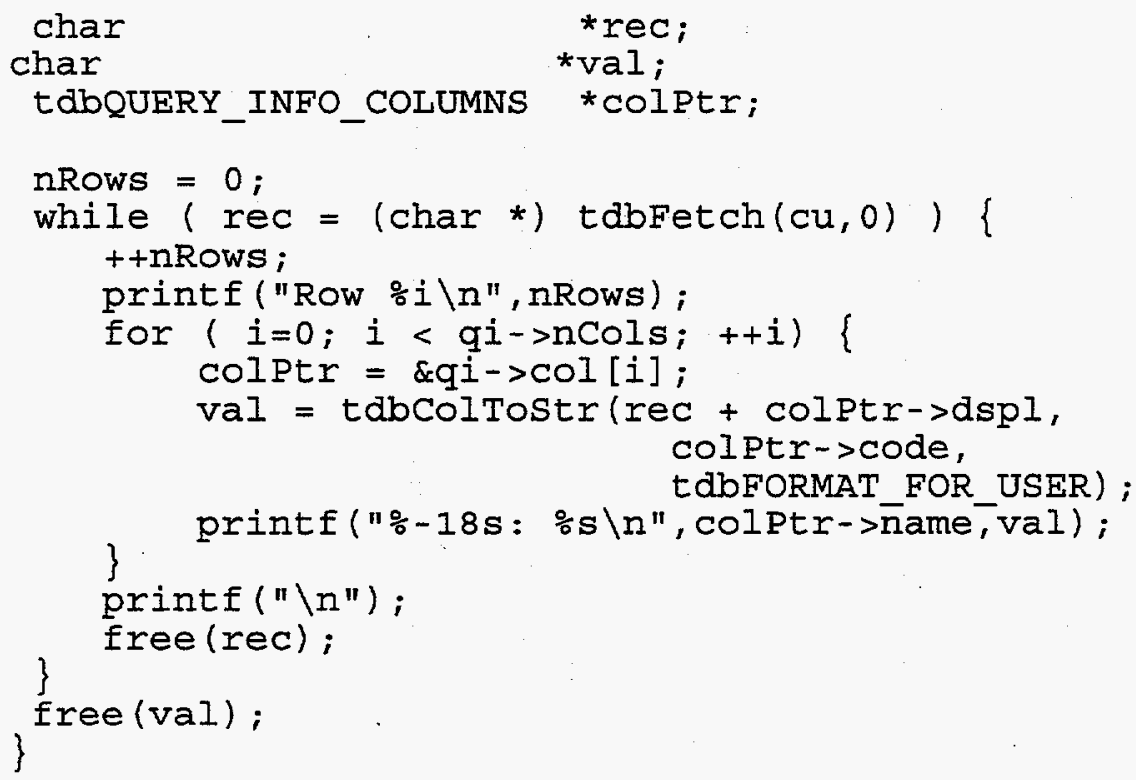




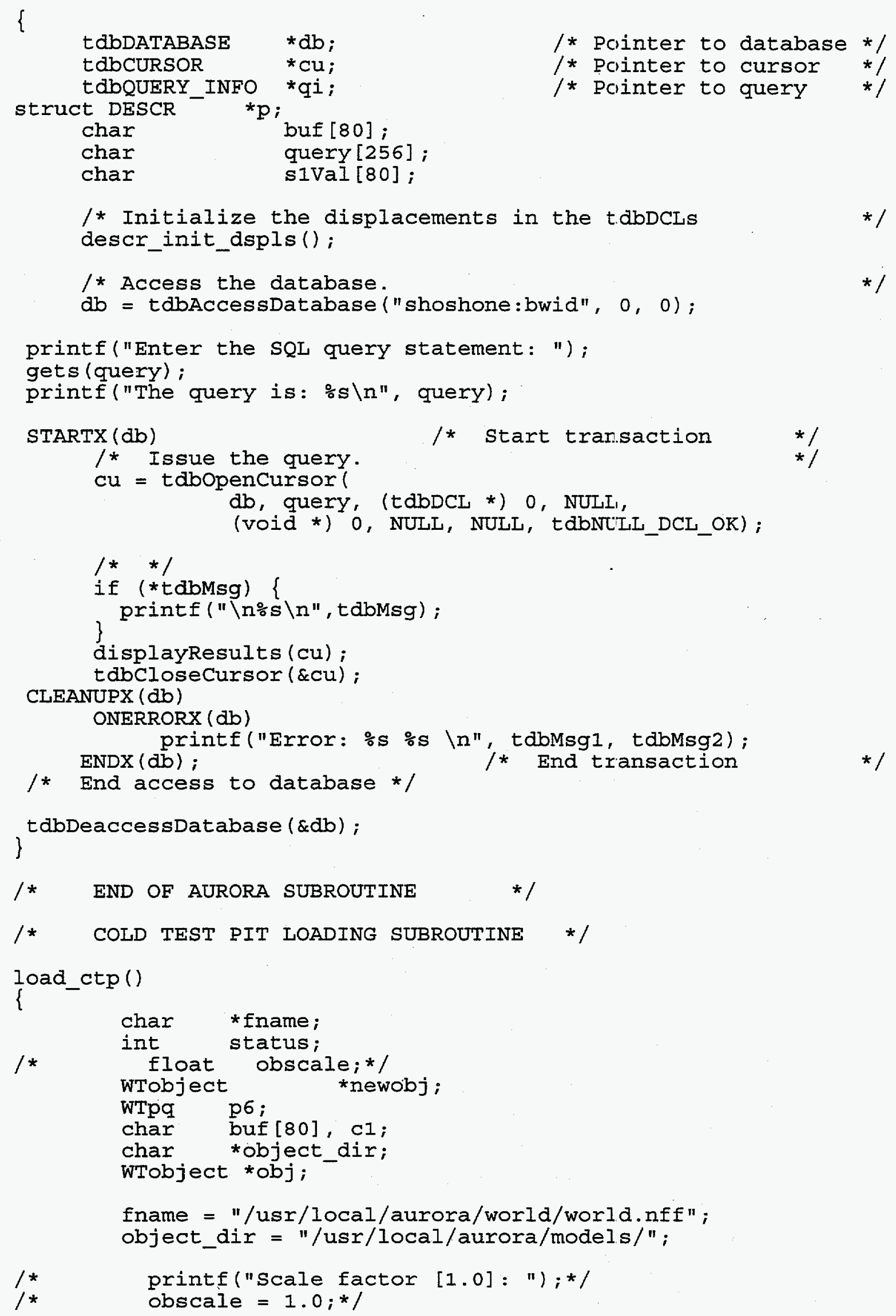




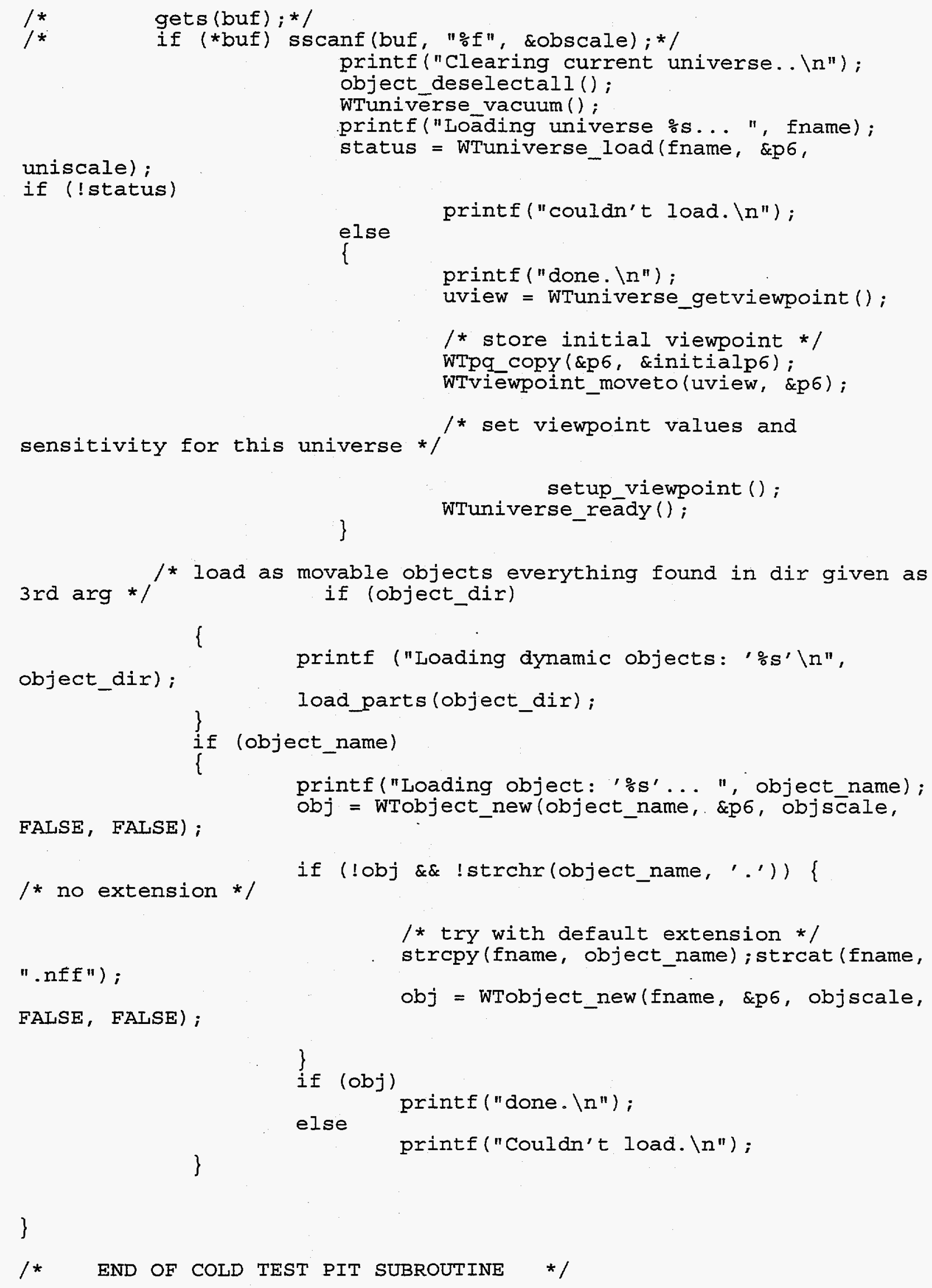




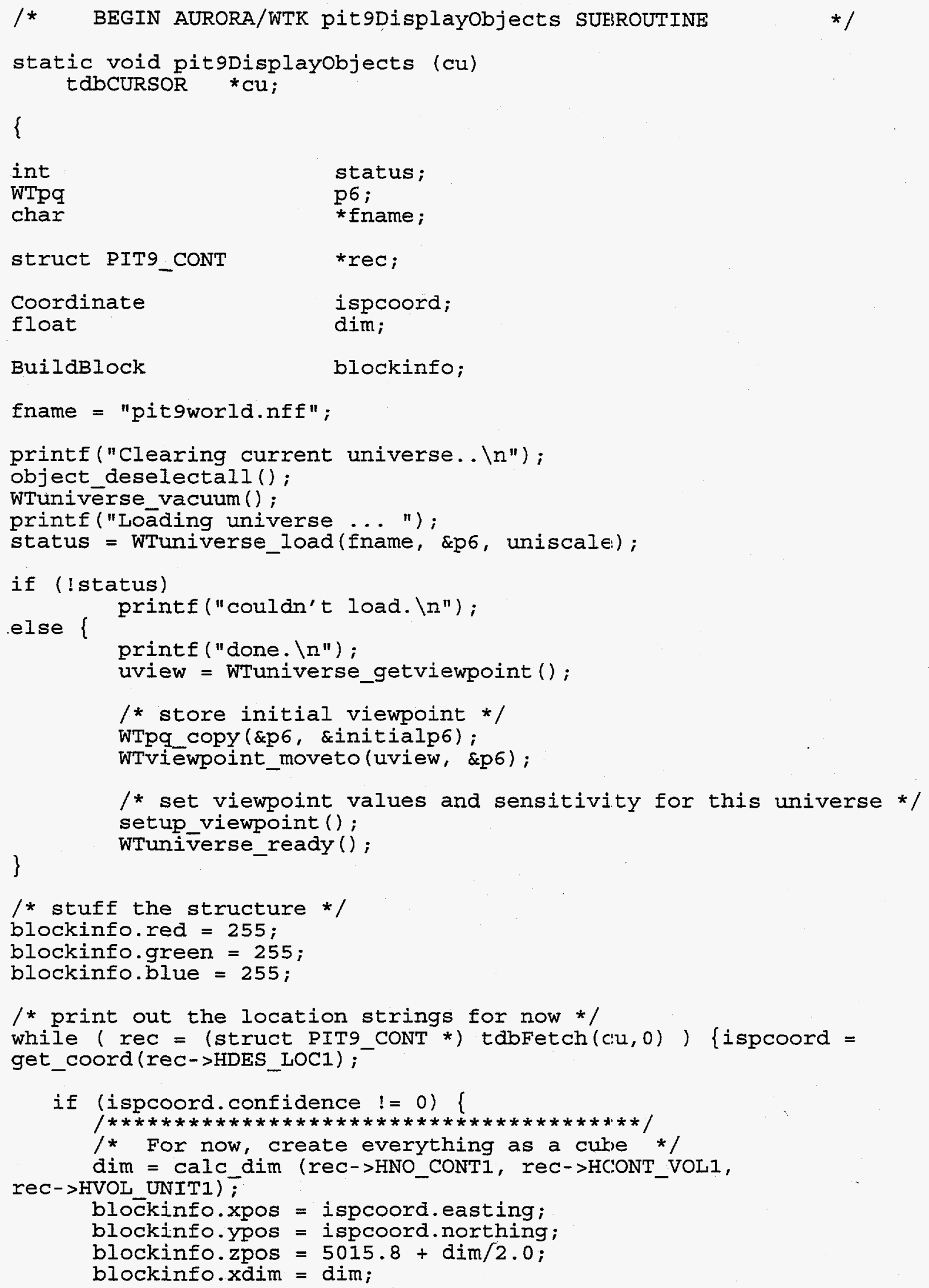




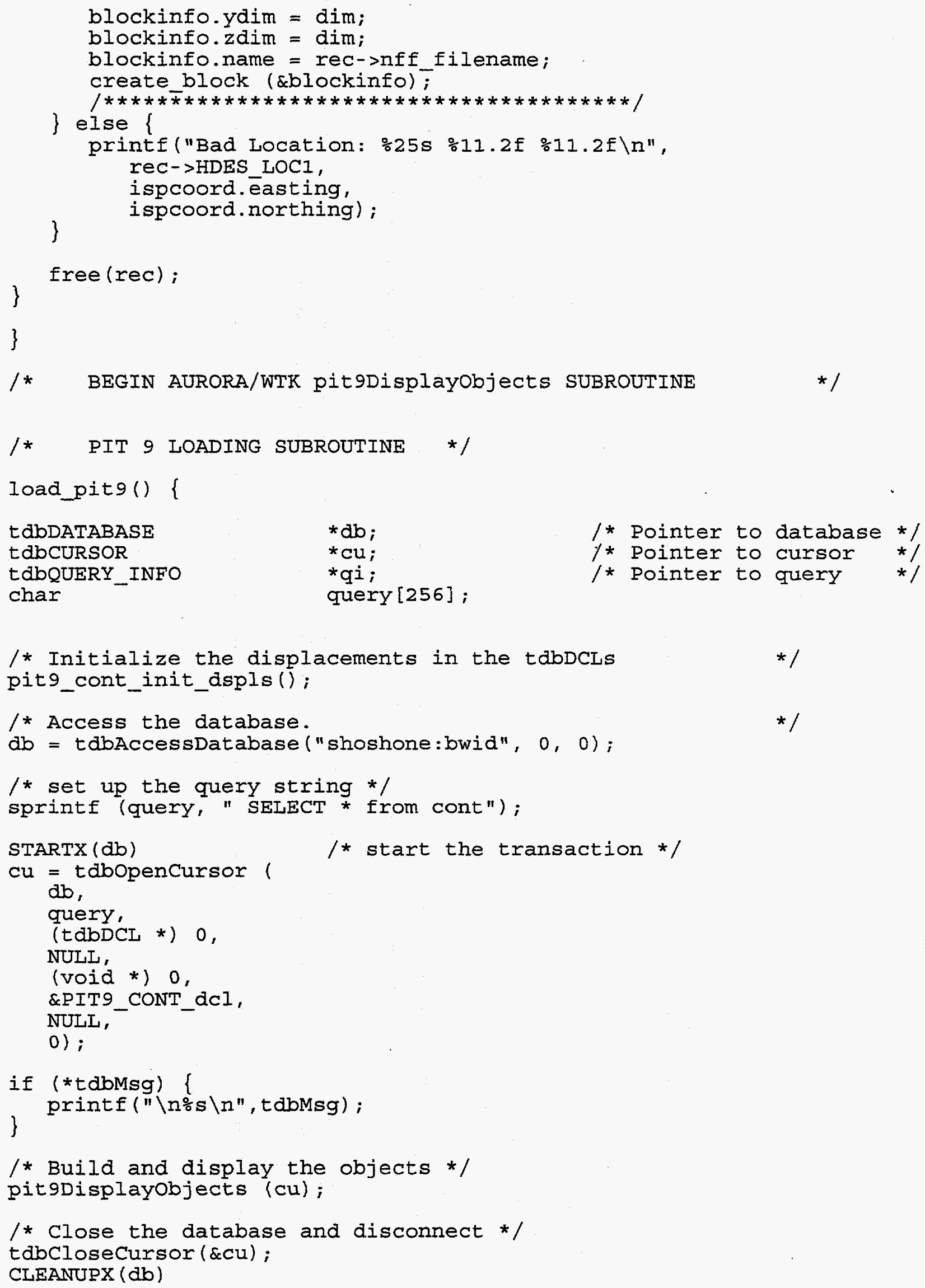




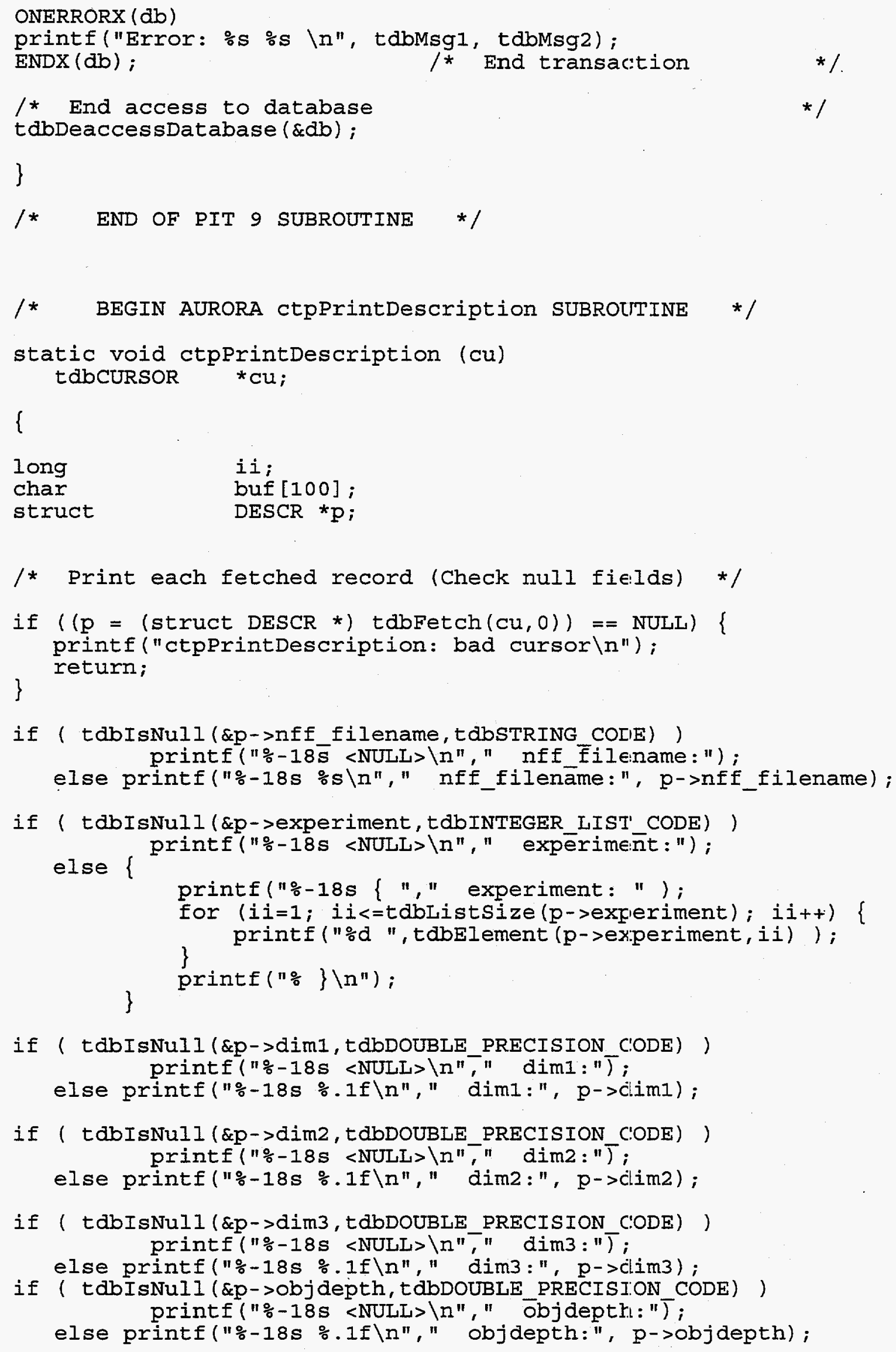




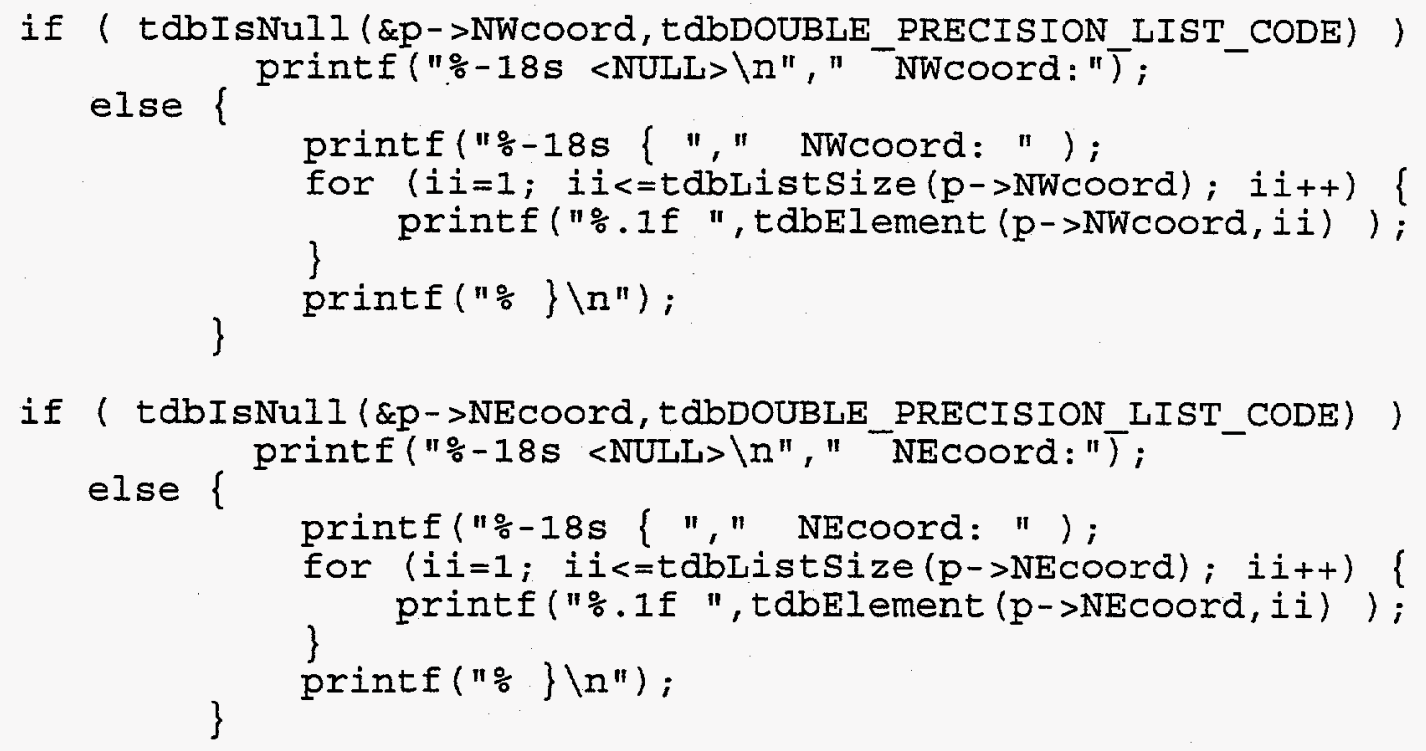

if ( tdbIsNuIl( $\& p->$ SWCOOrd, tdbDOUBLE_PRECISION_LIST_CODE) ) else \{ printf("\%-18s <NULL> In", " SWcoord:");

printf("-18s ("," SWcoord: ") ;

for ( $i=1 ;$ ii<=tdblistsize (p->Swcoord); iit+) \{

if ( tabIsNull ( $\&$ p->CENTERCoord, tdbDOUBLE_PRECISION_LIST_CODE) ) else \{ printf("\%-18s <NULL>In"," CENTERCOOrd:");

printe("응-18s \{ "," CENTERCoord: ");

for ( $i=1 ; i i<=$ tdbisistsize (p->CENTERCoord); iit+) \{ \} printf("\%.lf ",tdbElement (p->CENTERcoord, ii) );

if (tdbIsNull( \&p->radius,tdbDOUBLE_PRECISION_CODE) ) printf("\%-18s <NULL> $\backslash \mathrm{n} ", "$ radius: "T;

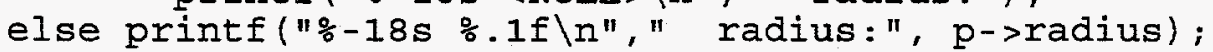

if (tdbIsNulI ( $\& p$->description, tdbSTRING_CODE) ) printf("\%-18s <NULL> \n"," description:"); else printf("\%-18s $\% s \backslash n "$ " description:", p->description);

if (tdbIsNull ( $\& p$->notes,tdbSTRING_CODE) ) 


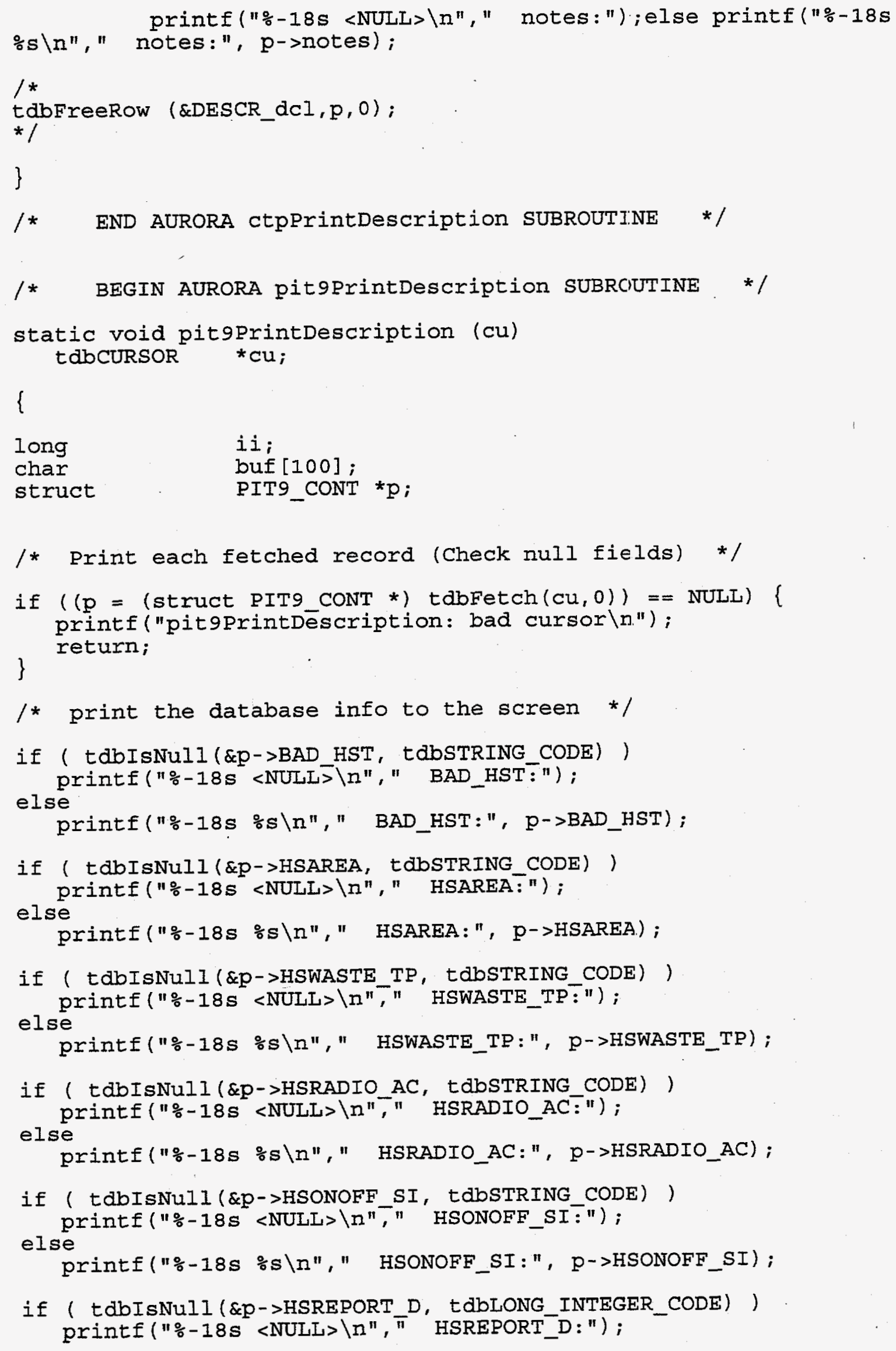


else printf ("\%-18s \%ld $\backslash n ", "$ HSREPORT_D:", p->HSREPORT_D);

if (tdbIsNuII ( $\& \mathrm{p}->$ HSREPORT_T, tdbINTEGER_CODE) ) printf ("\%-I8s <NULL> In", " HSREPORT_T:"); else printf ("\%-18s $\% d \backslash n ", "$ HSREPORT_T:", p->HSREPORT_T);

if ( tdbIsNuII ( $\& p->$ HSWASTE_DE, tdbSTRING_CODE) ) printf ("\%-I8S <NULL> \n"," HSWASTE_DE:") ; else printf("\%-18s \%s $\backslash n ", "$ HSWASTE_DE:", p->HSWASTE_DE);

if (tdbIsNuII ( $\& \mathrm{p}->$ HTYPE_CONT, tdbSTRING_CODE) ) printe $(" \circ-18 \mathrm{~s}<$ NULI> $\backslash \overline{\mathrm{n}} ", "$ HTYPE_CONT" ") ; else printf ("\%-18s \%s $\backslash n ", "$ HTYPE_CONT:", p->HTYPE_CONT);

if ( tabIsNull ( $\& p->$ HNO_CONT1, tdbINTEGER_CODE) )

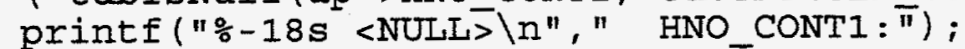
else printf ("\%-18s \%d $1 \mathrm{n} ", "$ HNO_CONT1:", p->HNO_CONT1) ;

if ( tdbIsNull ( $\& p->$ HCONT_VOL1, tdbINTEGER_CODE) ) printf ("\%-18s <NULL> $\backslash \overline{\mathrm{n}} ", "$ HCONT_VOLI:"); else printf ("\%-18s $\% d \backslash n ", "$ HCONT_VOL1:", p->HCONT_VOL1);

if ( tabIsNull ( $\& \mathrm{p}->$ HVOL_UNITI, tdbSTRING_CODE) ) printf ("\%-18s <NULL> Tn", " HVOL_UNIT1:"); else printf("\%-18s \%s $\backslash n ", "$ HVOL_UNIT1:", p->HVOI_UNIT1);

if ( tabIsNull (\&p->HSOLID_DS1, tdbSTRING_CODE) ) printf("\%-18s <NULL> \n"," HSOLID_DSI:"); else printf ("\%-18s \%s $\backslash \mathrm{n} ", "$ HSOLID DS1:", p->HSOLID_DS1); if (tabIsNull ( \&p->HDES LOC1, tdbSTRING_CODE) ) printf ("\%-18s <NULI>Tn"," HDES_LOC1""); else printf("\%-18s \%s $\backslash n ", "$ HDES_LOC1:", p->HDES_LOC1);

if ( tabIsNull (\&p->HDES_DAT1, tdbLONG_INTEGER_CODE) ) printf("\%-18s <NULL>Tn"," HDES_DATTI:"); else printf ("\%-18s \%ld $\backslash n ", "$ HDES_DAT1:", p->HDES_DAT1);

if ( tabIsNuIl ( $\& p->T R U$, tdbSTRING_CODE) ) else printf ("\%-18s <NULL> \n"," TRU:" ); printe("\%-18s \%s $\backslash n ", "$ TRU:", p->TRU)；

if ( tabIsNuII (\&p->HDES_LOC, tdbSTRING_CODE) ) else printe ("\%-18s <NULL> \n"," HDES_LOC:"); printf("\%-18s \%s $\backslash \mathrm{n} ", "$ HDES_LOC:", p->HDES_LOC);

if ( tabIsNulI (\&p->DOWNLOAD, tdbSTRING_CODE) ) printe ("\%-18s <NULL> \n"," DOWNLOAD:"); else printe ("\%-18s $\% s \backslash n ", "$ DOWNLOAD:", p->DOWNLOAD)； 


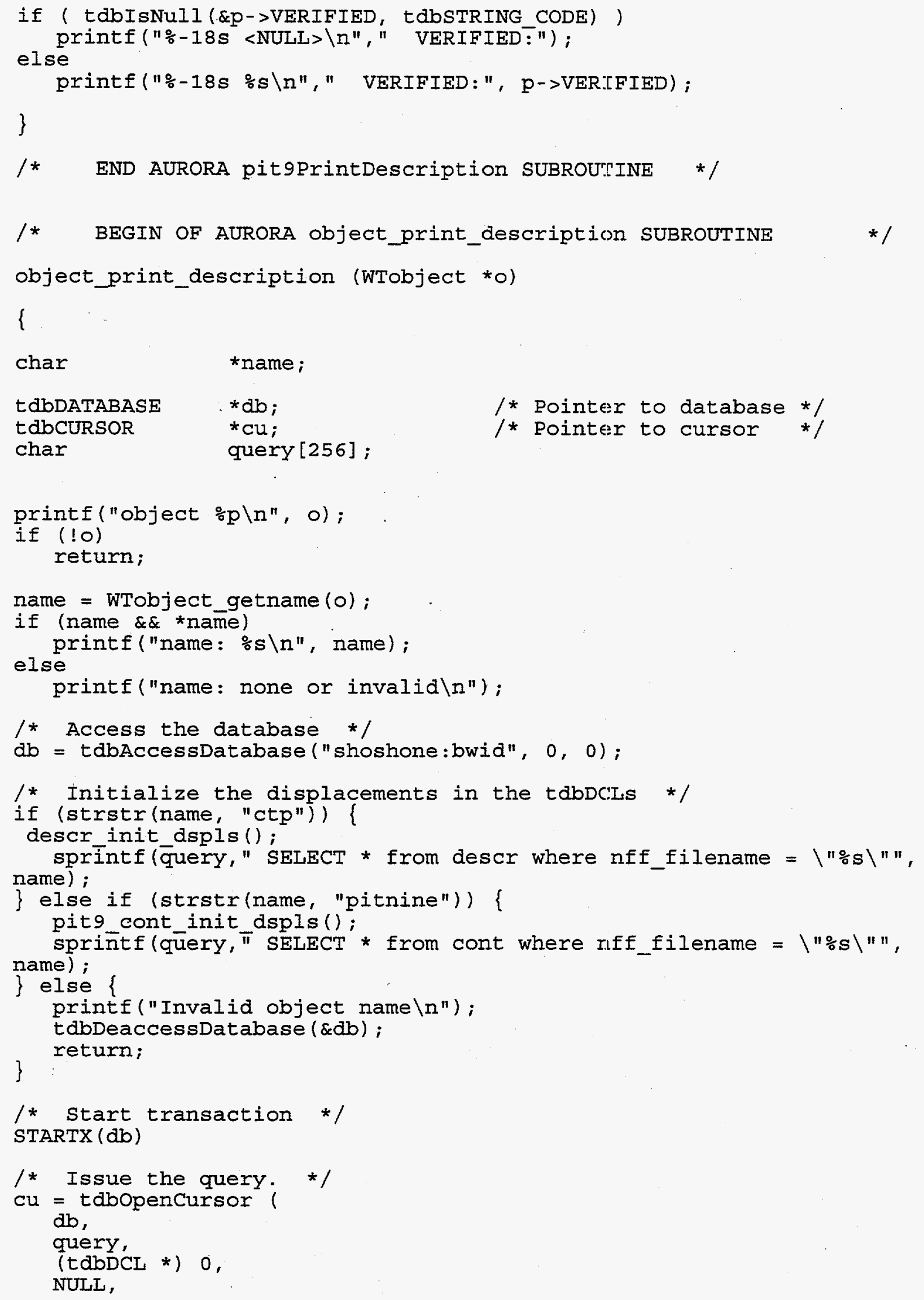




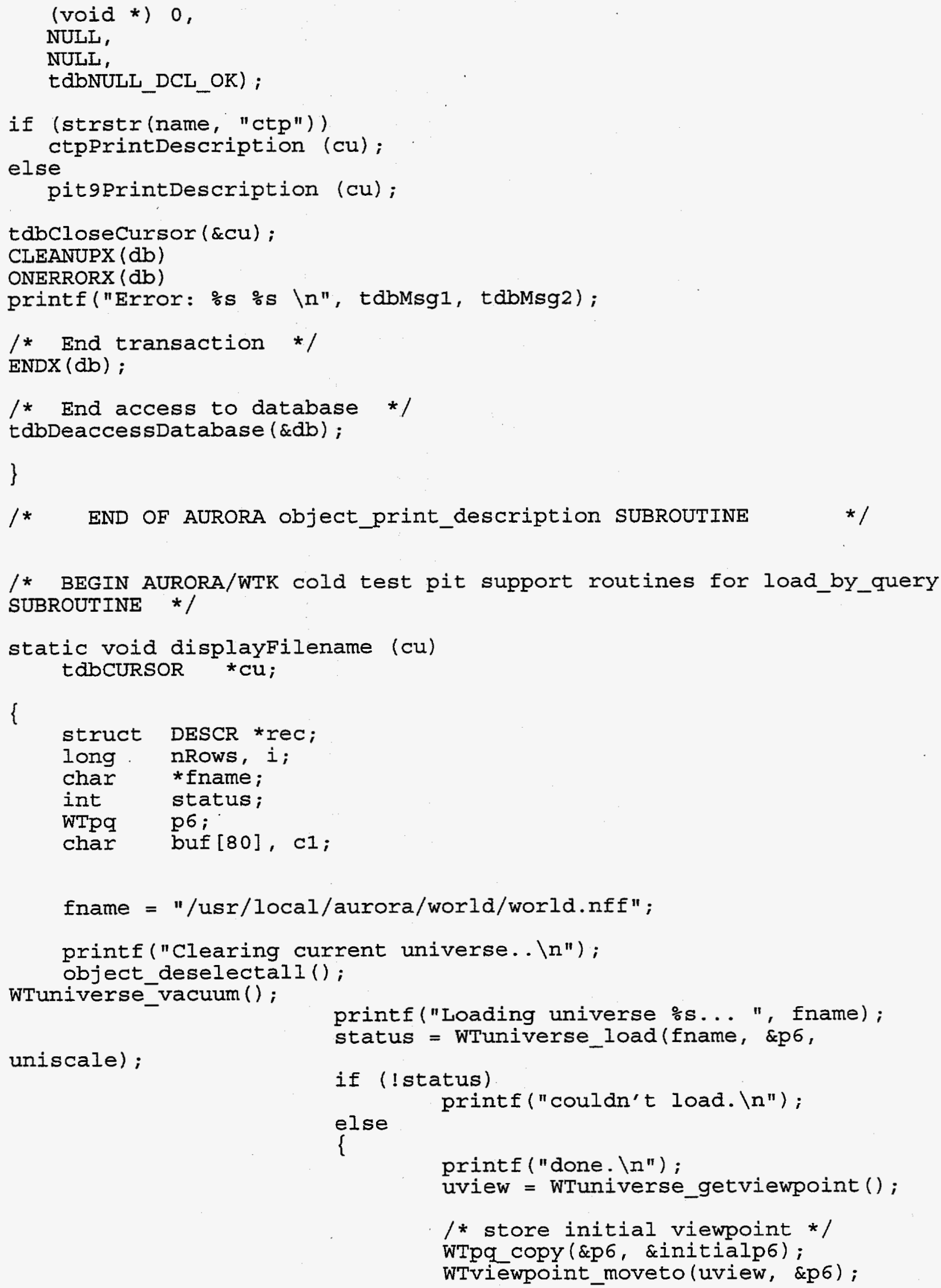


sensitivity for this universe $* / /$ set viewpoint values and

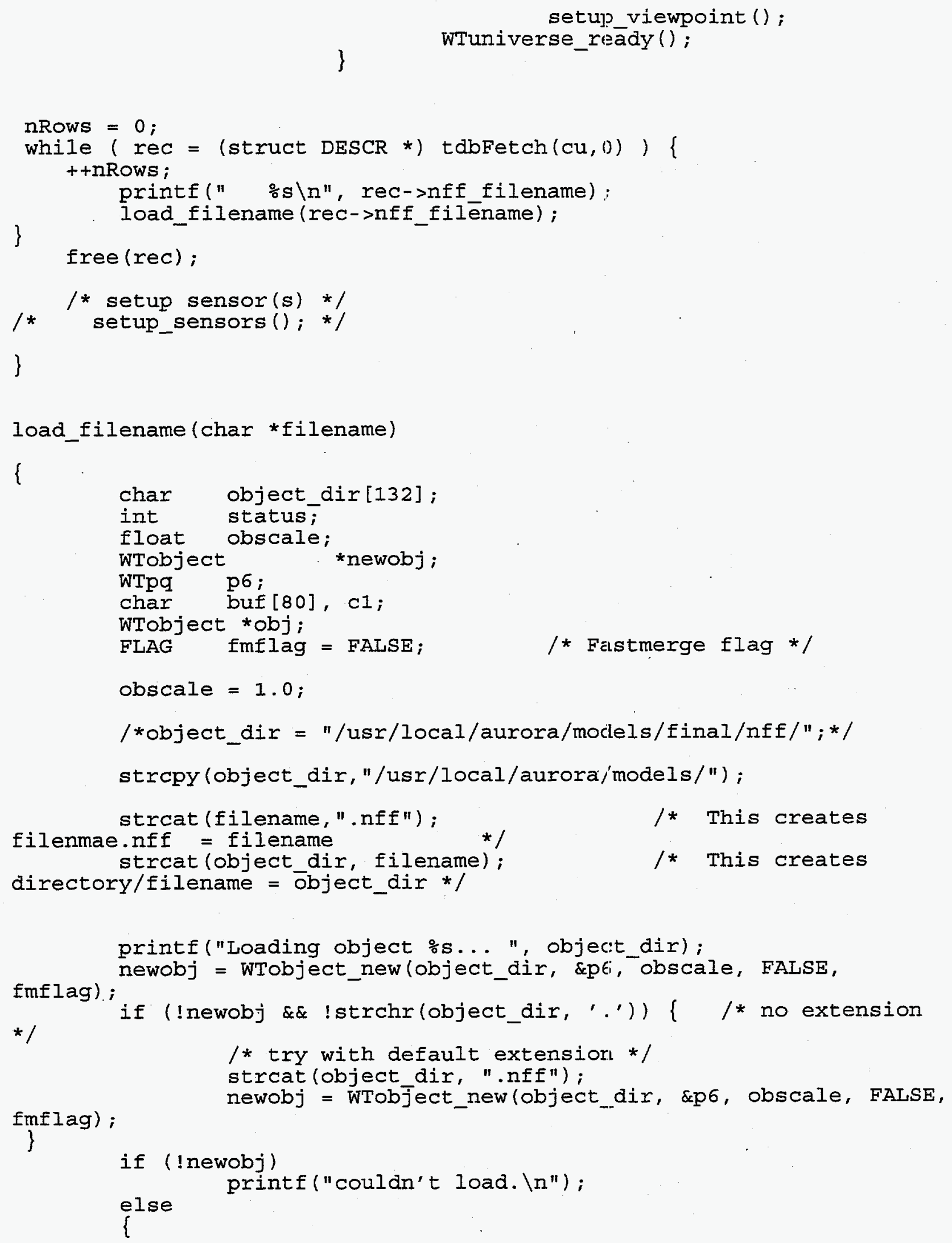




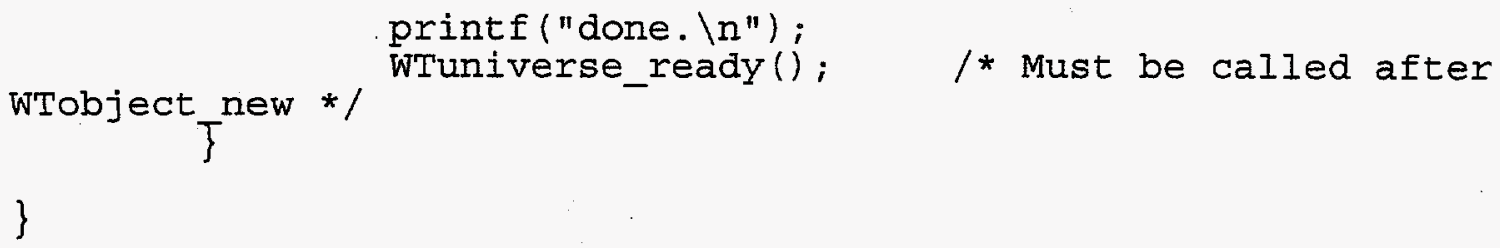

tdODATABASE

tObCURSOR

tObQUERY_INFO

char

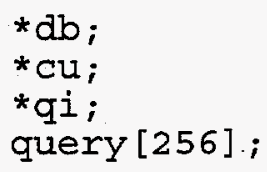

/* Pointer to database */

/* Pointer to cursor */

$/ *$ Pointer to query

/* Access the database.

$d b=$ tdbAccessDatabase ("shoshone:bwid", 0,0$)$;

*

printf("Enter the SQL query statement: ");

gets (query);

printf("The query is: \%s $\backslash n "$, query);

/* Build and display the objects

if (strstr(query, "from descr")) \{ descr_init_dspls();

\} else if́ (stristr(query, "from cont")) \{ pit9_cont_init_dspls();

\} else T printe ("Must select from the descr or cont databases $\backslash n$ ") ; tdbDeaccessDatabase (\&db);

\} return;

/* Start the transaction */ $\operatorname{STARTX}(d b)$

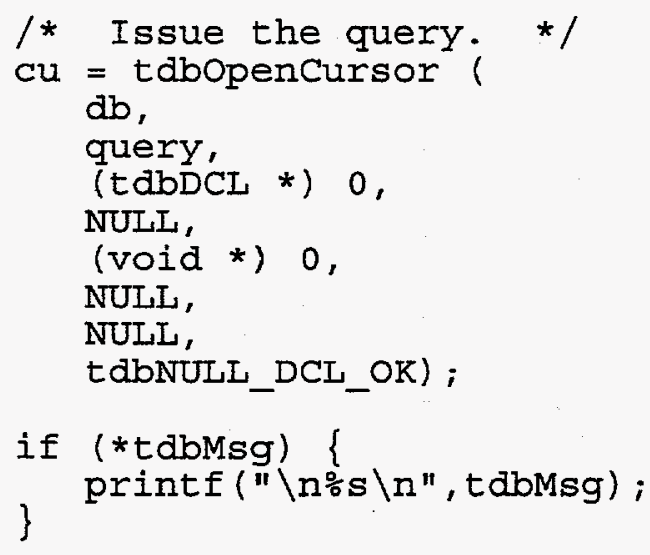


/* Build and display the objects */

if (strstr(query, "from descr")) ( printf("Selecting from ctp $\backslash n$ "); displayFilename (cu);

\} else if (strstr(query, "from cont")) \{ printf("selecting from pit $9 \backslash \mathrm{n} "$ );

\} else pit9Displayobjects (cu);

printf("Must select from the descr or cont databases $n "$ ")

/* Close and disconnect from the database */

tdbcloseCursor (\&cu);

CLEANUPX ( $d b)$

ONERRORX $(d b)$

printf("Error: $\frac{\circ}{8} \div s \backslash n "$, tdbMsg1, tdbMsg2);

/* End transaction */

$\operatorname{ENDX}(a b)$;

/* End access to database */

tdbDeaccessDatabase ( $\& d b)$;

\}

/* END OF AURORA/WTK load_by_query SUBROUTINE 
Appendix G

Source Code 
G-2 


\section{Appendix G}

\section{Source Code}

\section{G-1. INTRODUCTION}

This appendix contains the source code required to generate objects in the WorldToolKit model from data base information accessed by WTK. Also given is the source code for utility functions that compute coordinate locations in Idaho State Plane coordinates based on location fields in the data base record and generate cube objects based on information in the data base record.

\section{G-2. SOURCE CODE}

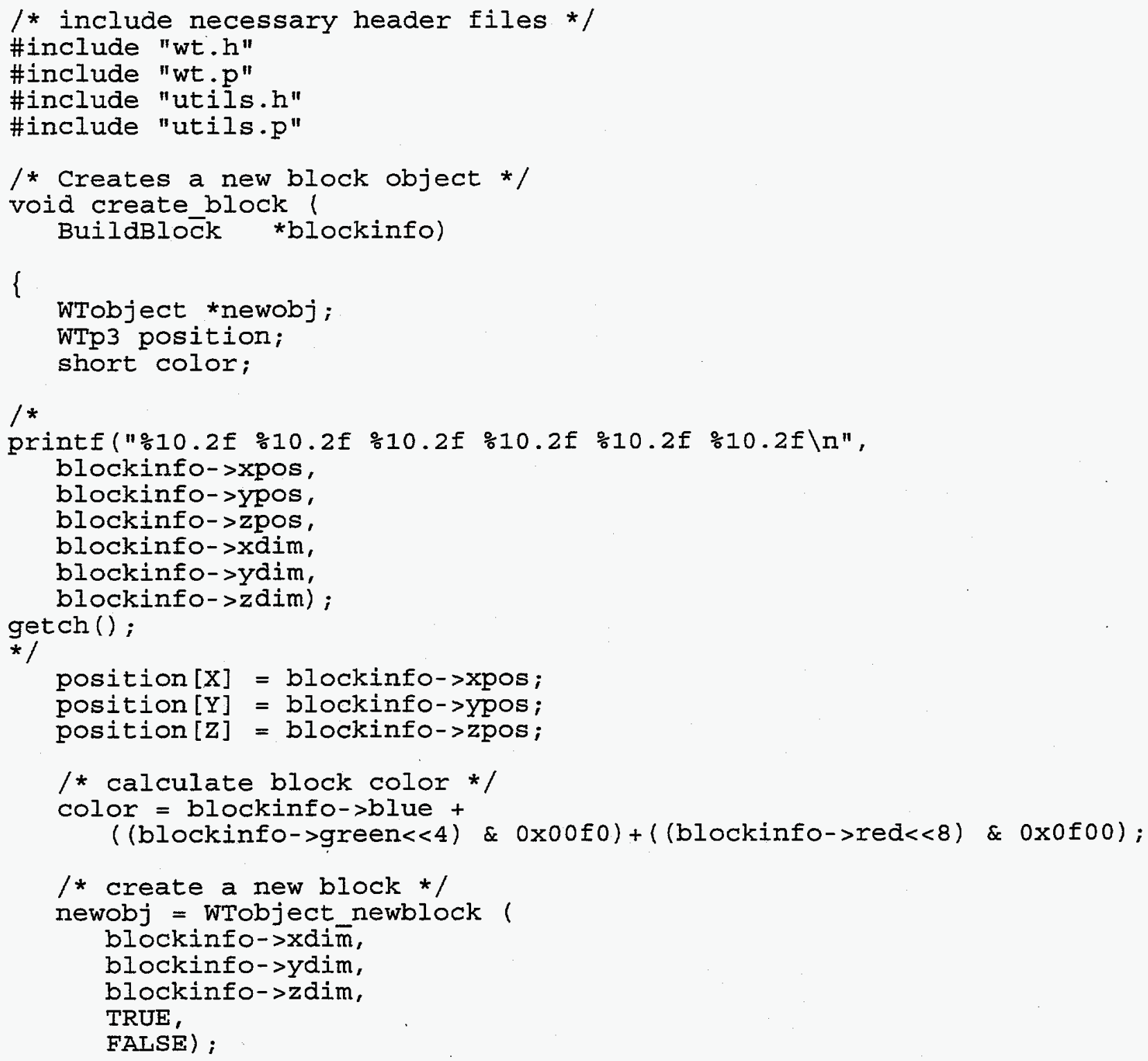




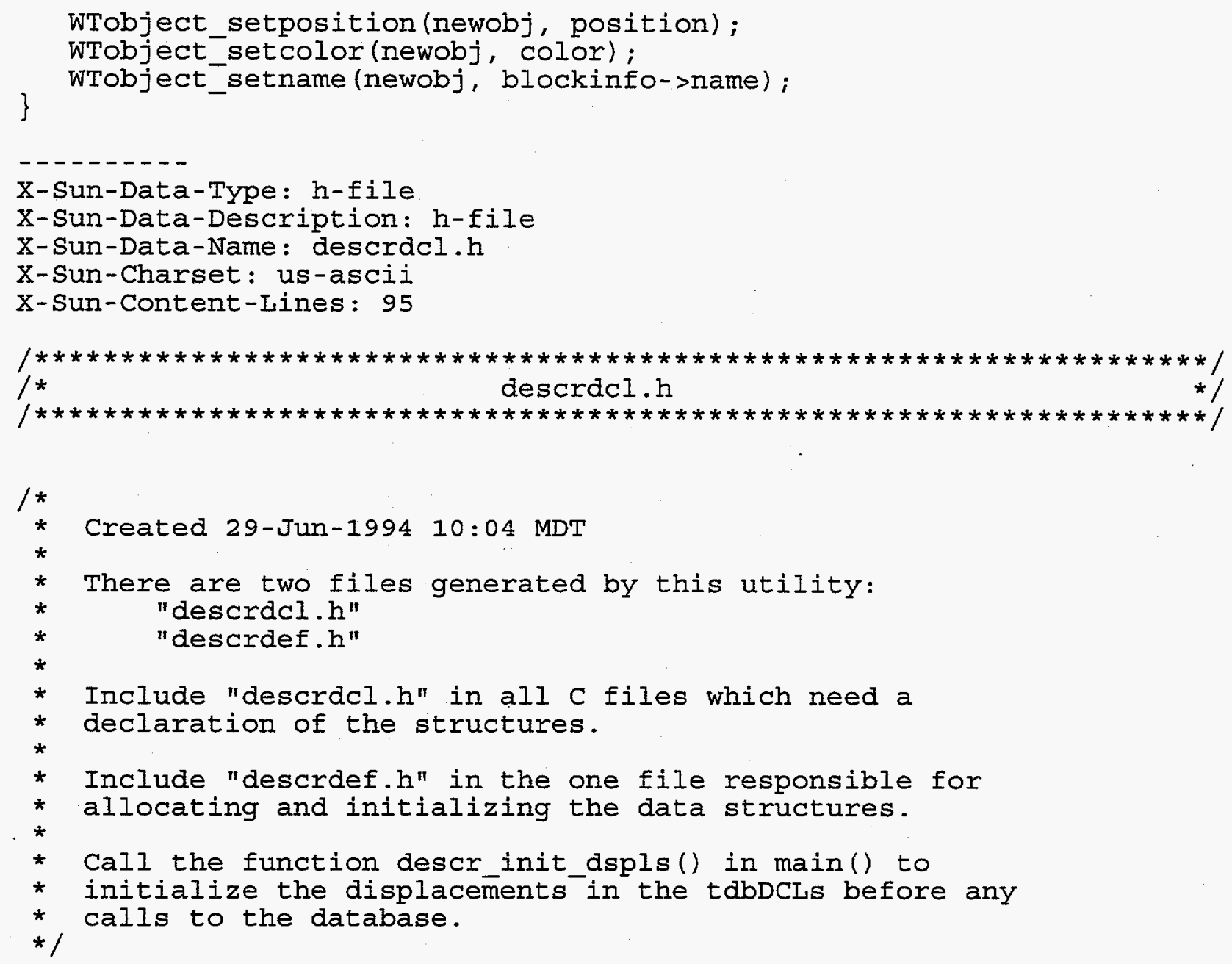

\#ifndef DESCR_DCL_HEADER \#define _DESCR_DCI_HEADER

\#ifndef DECLARED_DESCR_REFS \#define DECLARED_DESCR_REFS

/* Declare references for all structs */ tdbDECLARE_REFERENCE (DESCR); 


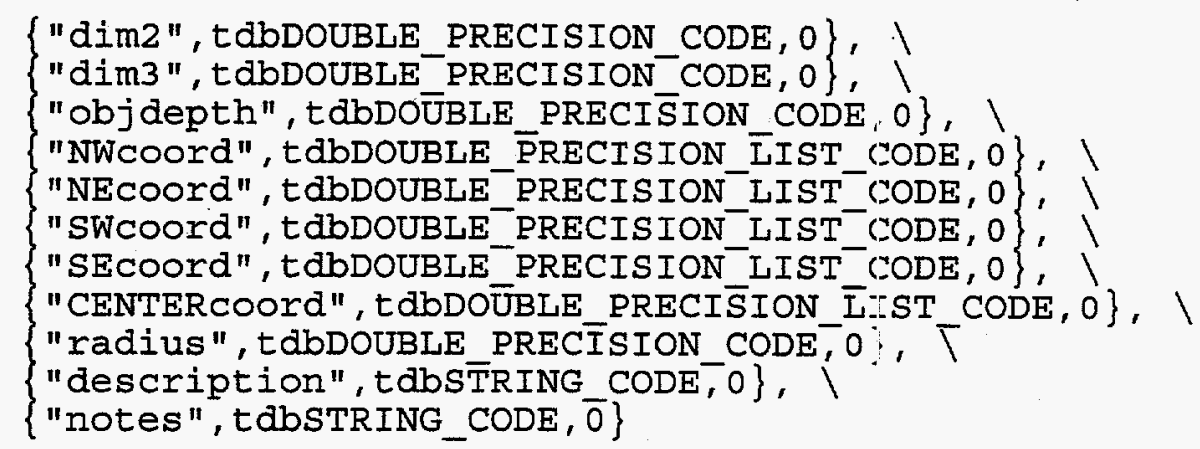

\#define DESCR_N_COLUMNS 15

struct DESCR \{

\# define DESCR_COLUMN_VARIABLES ।

tdbLONG INTEGER objId; 1

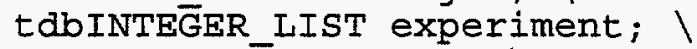

char * nff-filename; I

tdbDOUBLE_PRECISION dim1;

tdbDOUBLE PRECISION dim2;

tdbDOUBLE-PRECISION $\operatorname{dim} 3$;

tdbDOUBLE_PRECISION objdepth;

tdbDOUBLE_PRECISION_LIST NWCOOId;

tdbDOUBLE ${ }^{-}$PRECISION LIST NECOOrd;

tdbDOUBLE ${ }^{-}$PRECISION IIST SWCOOrd;

tdbDOUBLE_PRECISION_LIST SECOOrd;

tdbDOUBLE_PRECISION_LIST CENTERCOORd; ।

tdbDOUBLE_PRECISION ${ }^{-}$radius; ।

char * description; 1

char * notes

DESCR_COLUMN_VARIABLES;

\}.;

\#endif $/ *$ tdbDEFINED_DESCR */

extern tdbCOLUMNS DESCR_cols [];

extern tdbDCL DESCR del;

extern char *DESCR Column list;

_tdbEXTERN_F(void DESCR_iñit_dspls, (tdbDCL*dcl) );

extern tabDCL *descr expansion dcls [] ;

\#endif $/ *$ DESCR_DCL_HEADER */

X-Sun-Data-Type: h-file

X-Sun-Data-Description: h-file

$\mathrm{X}$-Sun-Data-Name: descrdef.h

$\mathrm{x}$-Sun-Charset: us-ascii

$\mathrm{X}$-Sun-Content-Lines: 83

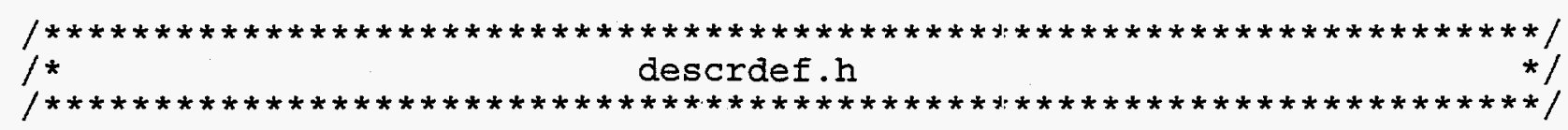




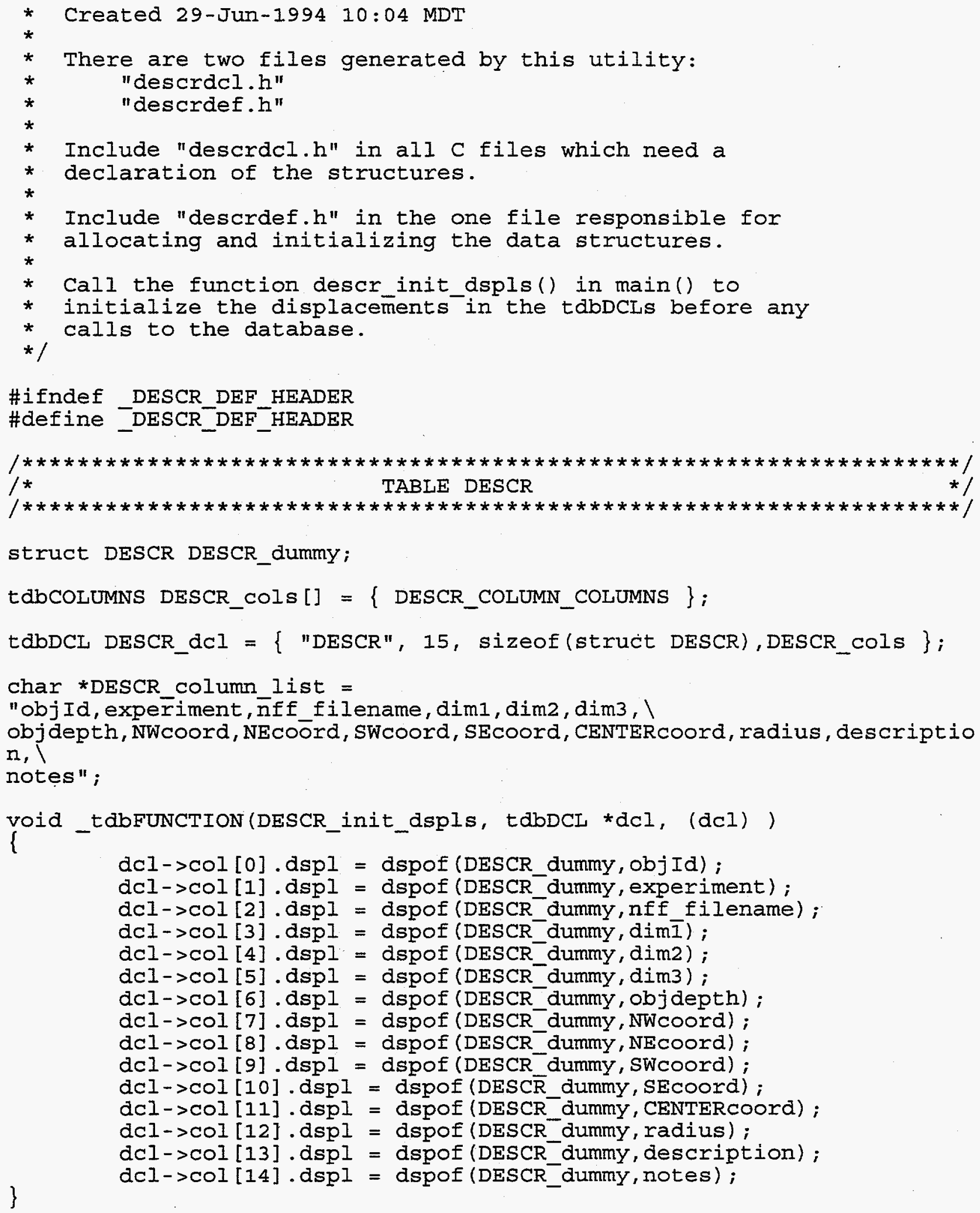

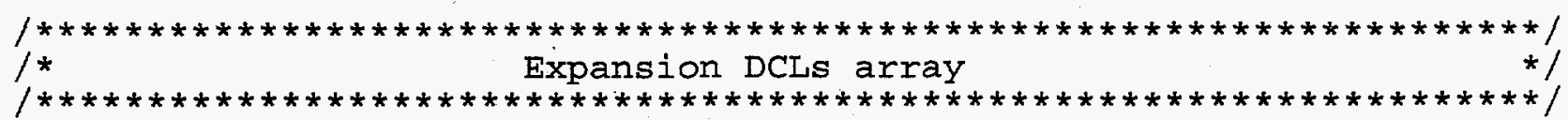




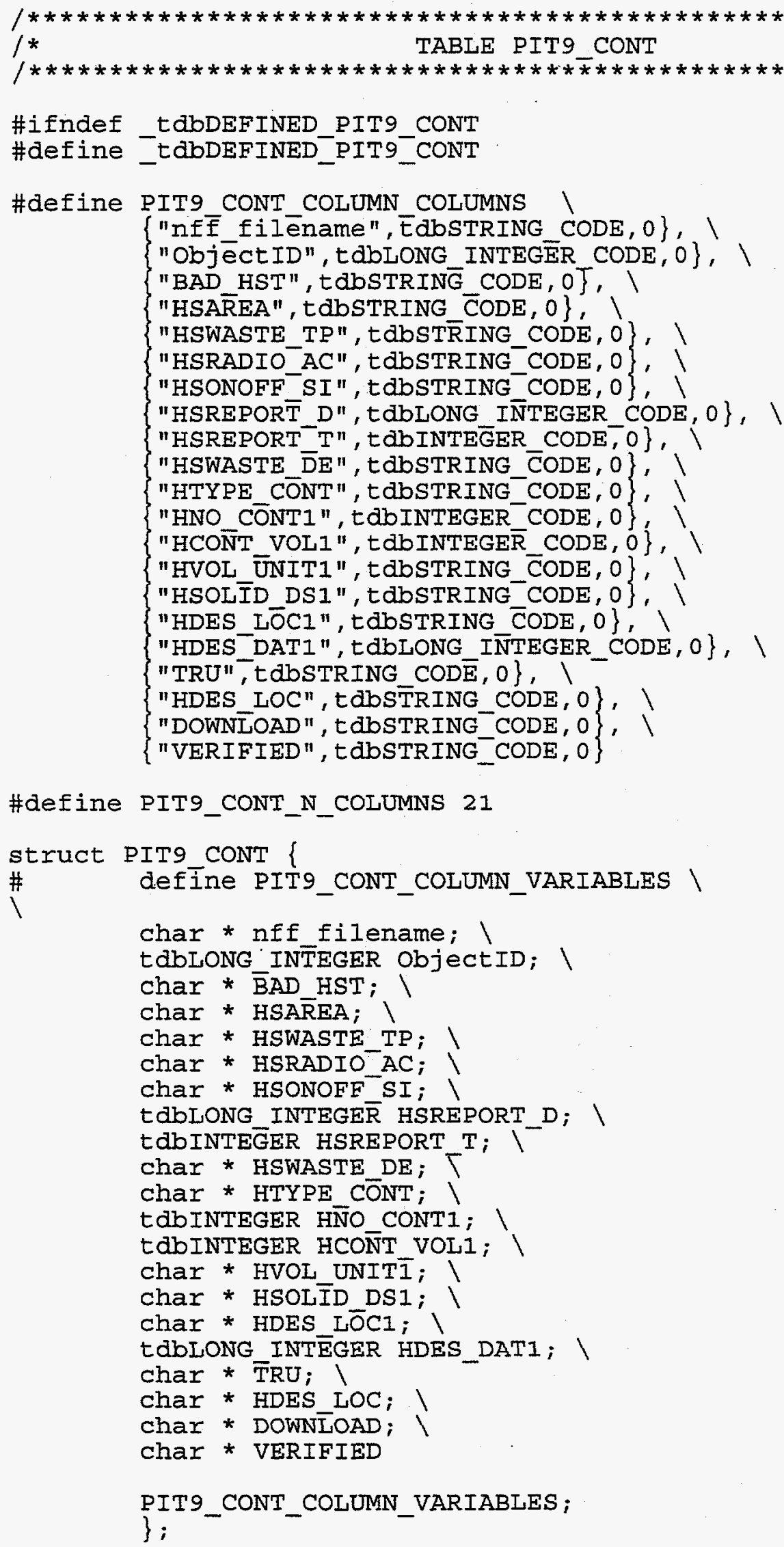




$$
\begin{aligned}
& \text { void_tdbFUNCTION(PIT9_CONT_init_dspls, tdbDCL *dcl, (dcl) ) } \\
& \text { dcI->col[0].dspl = dspof (PIT9_CONT_dummy,nff_filename) } ; \\
& \text { dcl->COl[1].dspl = dspof (PIT9_CONT_dummy, objectID); } \\
& \text { dcl->COI [2].dspl = dspof (PIT9_CONT_dummy,BAD_HST); } \\
& \text { dcl->col [3].dspl = dspof (PIT9 CONT dummy, HSA } \bar{R} E A) \text {; } \\
& \text { dcl->col [4].dspl = dspof (PIT9-CONT_dummy,HSWASTE_TP); } \\
& \mathrm{dcl}->\mathrm{COl}[5] . \mathrm{dspl}=\text { dspof (PIT9_CONT_dummy, HSRADIO_AC); } \\
& \text { dcl->COI [6].dspI = dspof (PIT9_CONT_dummy, HSONOFF_SI); } \\
& \mathrm{dcl}->\operatorname{COl}[7] \text {.dspl = dspof (PIT9-CONT dummy, HSREPORT D); } \\
& \text { dcl->col [8].dspl = dspof (PIT9_CONT_dummy, HSREPORT_T); } \\
& \text { dcl->col [9] .dspl = dspof (PIT9_CONT_dummy, HSWASTE } \bar{D} E \text { ); } \\
& \text { del->COl[10].dspl = dspof (PIT } \overline{9} \text { CONT_dummy, HTYPE } \overline{C O N T}) ; \\
& \text { dcl->col[11].dspl = dspof (PIT9_CONT_dummy, HNO_CONT1); } \\
& \text { dcl->COl [12].dspl = dspof (PIT9_CONT_dummy, HCON̄T_VOL1); } \\
& \mathrm{dcl}->\mathrm{COI} \text { [13].dspl = dspof (PIT9_CONT_dummy, HVOI_UTNIT1); }
\end{aligned}
$$

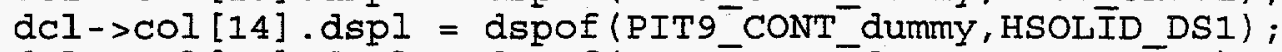

$$
\begin{aligned}
& \text { dcl->COl [15].dspl = dspof (PIT9 CONT dummy, HDES LŌC1); } \\
& \text { dcl->col [16].dspl = dspof (PIT9_CONT_dummy, HDES_DAT1); } \\
& \text { dcl->COl[17].dspl = dspof (PIT9-CONT-dummy, TRU) } ; \\
& \text { dcl->COI [18].dspI = dspof (PIT9_CONT_dummy, HDES_LOC); } \\
& \text { dcl->col[19].dspl = dspof (PIT9-CONT_dummy, DOWNL̄OAD); }
\end{aligned}
$$

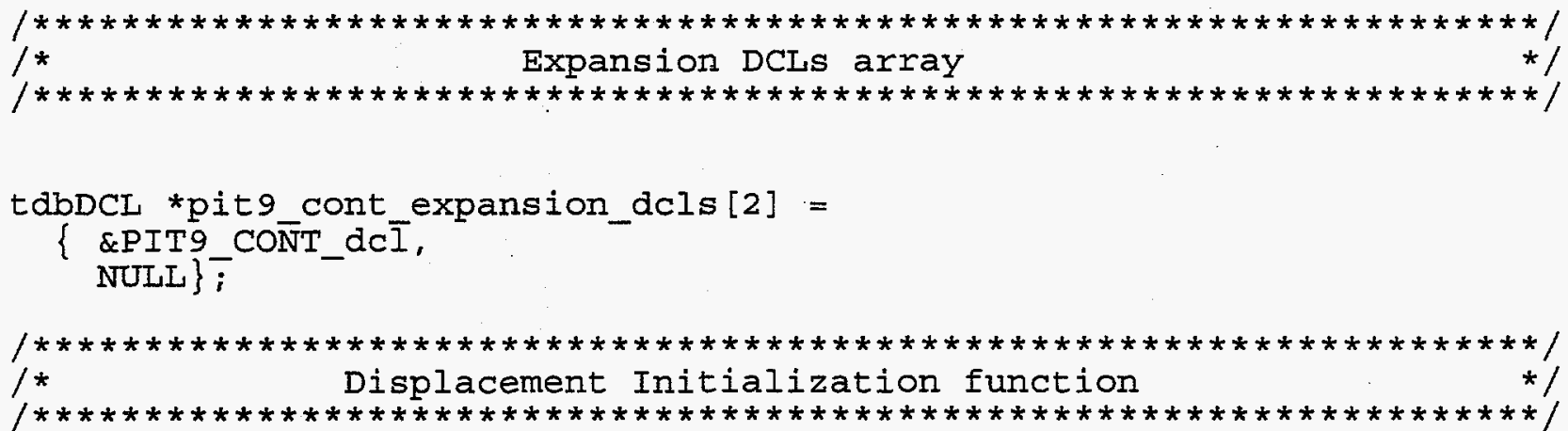


// The detail of the vertices can be as much as $10 \mathrm{ft}$ off

viewpos $0.0 \quad 0.0 \quad 0.0$ viewdir 0.00 .0 . 0.0
Pit9boundary

12

268420.23669933 .405030 .

$268295.18 \quad 669942.70 \quad 5030$.

268167.35669556 .985030 .

268266.31669564 .535030 .

268303.71669669 .755030 .

268333.75669662 .915030 .

$268420.23 \quad 669933.40 \quad 5015.80$

$268295.18 \quad 669942.70 \quad 5015.80$

$268167.35 \quad 669556.98 \quad 5015.80$

$268266.31669564 .53 \quad 5015.80$

$268303.71 \quad 669669.75 \quad 5015.80$

$268333.75 \quad 669662.91 \quad 5015.80$

20

3450 oxfoo both

$3 \quad 4 \quad 0 \quad 1$ oxfoo both

$\begin{array}{llllll}3 & 2 & 4 & 1 & 0 \times f 00 & \text { both }\end{array}$

$3 \quad 2 \quad 3 \quad 4$ oxfoo both

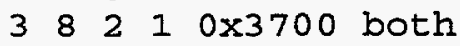

$37810 \times 3700$ both

310 I1 6 oxf00 both

$\begin{array}{lllllll}3 & 10 & 6 & 7 & 0 \times f 00 & \text { both }\end{array}$

$\begin{array}{lllll}3 & 8 & 10 & 7 & \text { oxfoo both }\end{array}$

$\begin{array}{lllll}3 & 8 & 9 & 10 & 0 \times f 00 \\ 3 & 60 t h\end{array}$

$360050 \times 3700$ both

$311650 \times 3700$ both

$310060 \times 8700$ both

$3711660 \times 8700$ both

$399320 \times 8700$ both

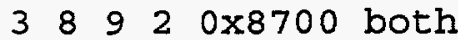

$\begin{array}{llllll}3 & 10 & 3 & 4 & 0 \times 0 f 0 & \text { both }\end{array}$

$\begin{array}{lllll}3 & 9 & 10 & 3 & 0 \times 0 f 0 \\ & & & \end{array}$

3 11 4 4 5 oxofo both

310114 oxofo both

-----.--.

X-Sun-Data-Type: c-file

X-Sun-Data-Description: c-file

$\mathrm{X}$-Sun-Data-Name: utils.c

$\mathrm{X}$-Sun-Charset: us-ascii

$\mathrm{X}$-Sun-Content-Lines: 517
$/ /$ top ofxoo is the color, in this case, Red

// L-side

// bottom

// R-side

// Back

$/ /$ Front

// little side

$/ /$ other little side
$/ /$ Number of vertices

$/ /$ Number of vertices

/* include necessary header files */

\#include <stdio.h>

\#include <math.h>

\#include <stalib.h>

\#include <string.h>

\#include "utils.h"

\#include "utils.p"

/* function protypes */

float calc_average (

char

*str); 


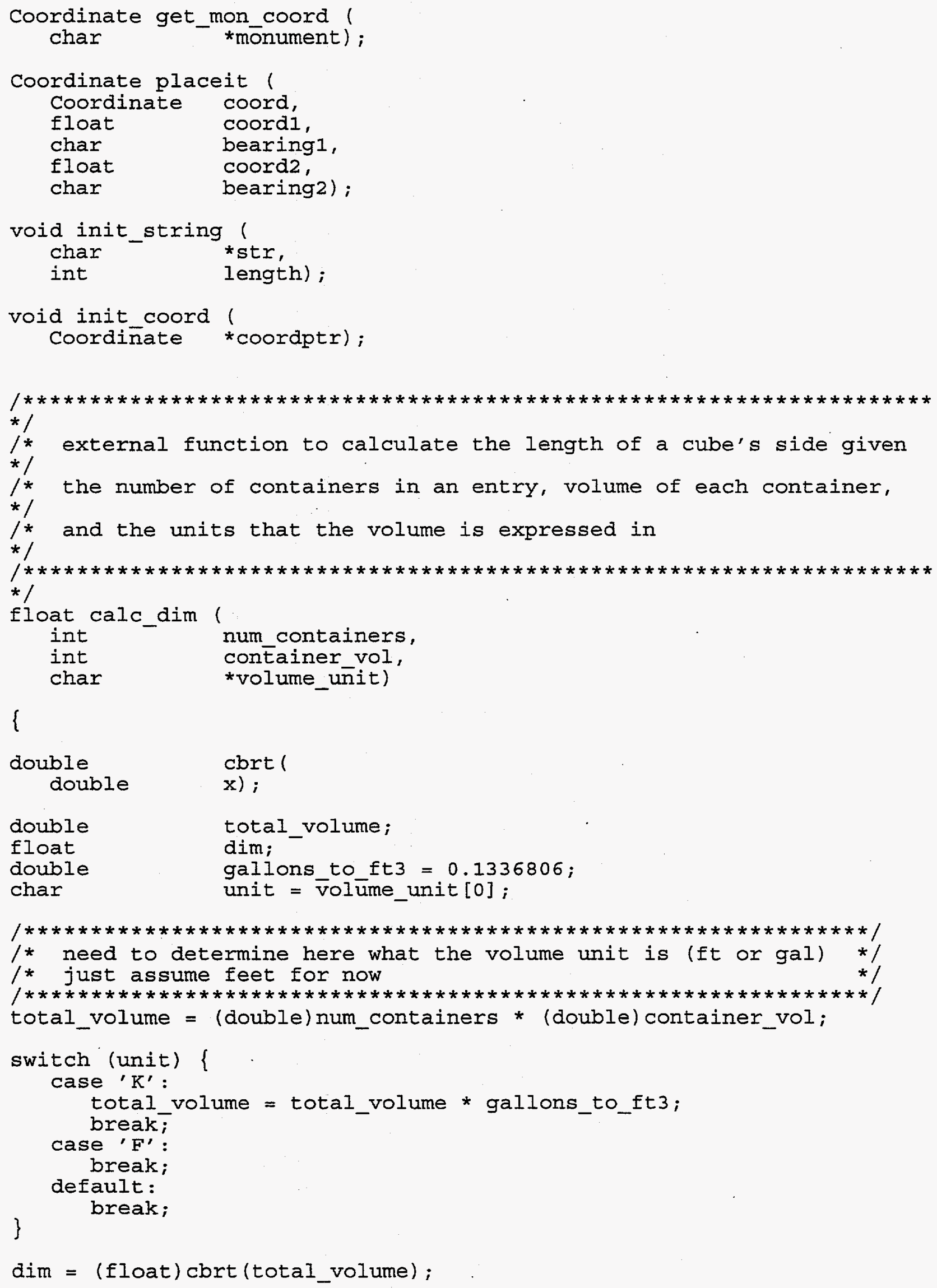


return (dim);

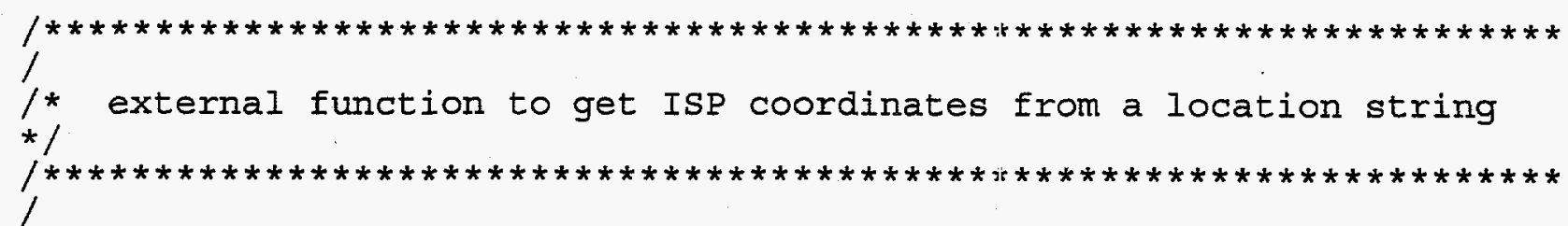
Coordinate get_coord (

char

*location)

\{

int $\quad i, j, m, n, o, p$;

char loccopy[25], *mychar, pit[10], coord1[10], coord2[10], monument [10], *pitptr, *coordlptr, *coordzptr, *monumentptr;

char

char

int

float

Coordinate

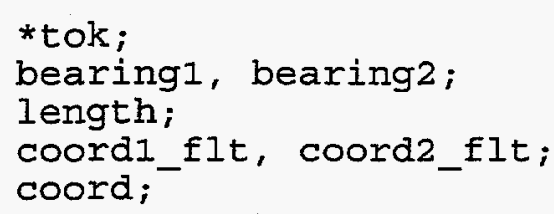

/* initialize the object coordinates structure */

init_coord (scoord);

/* initialize the bearing string */

bearing $1=' \backslash 0^{\prime} ;$

bearing $2=10^{\prime} ;$

/* initialize the index variables */

$\mathrm{m}=\mathrm{n}=0=\mathrm{p}=0$;

/* make a local copy of the location string and find its length */ strcpy (loccopy, location);

$i=\operatorname{strlen}($ loccopy);

mychar = Ioccopy;

/* find the pit, coord, and monuments in the string */

for $(j=0 ; j<i ; j++$, mychar +$)\{$

/* find the end of the pit field */

if $\left(\left(*\right.\right.$ mychar $\left.==\prime g^{\prime}\right)$ \&\& $\left.(j<=4)\right)$ $\mathrm{m}=\mathrm{j}$;

* $/$ * find the end of the coordinate 1 field, including the bearing

if $\left(\left(\left(*\right.\right.\right.$ mychar $\left.==\prime N^{\prime}\right) \|\left(*\right.$ mychar $\left.==\prime S^{\prime}\right)||$

$\left(*\right.$ mychar $\left.==' E^{\prime}\right) \mid\left(*\right.$ mychar $\left.==' W^{\prime}\right)$ ) \&\&

$(\mathrm{n}==0)$ )

$\mathrm{n}=\mathrm{j} ;$

*/ $/$ find the end of the coordinate 2 field., including the bearing 


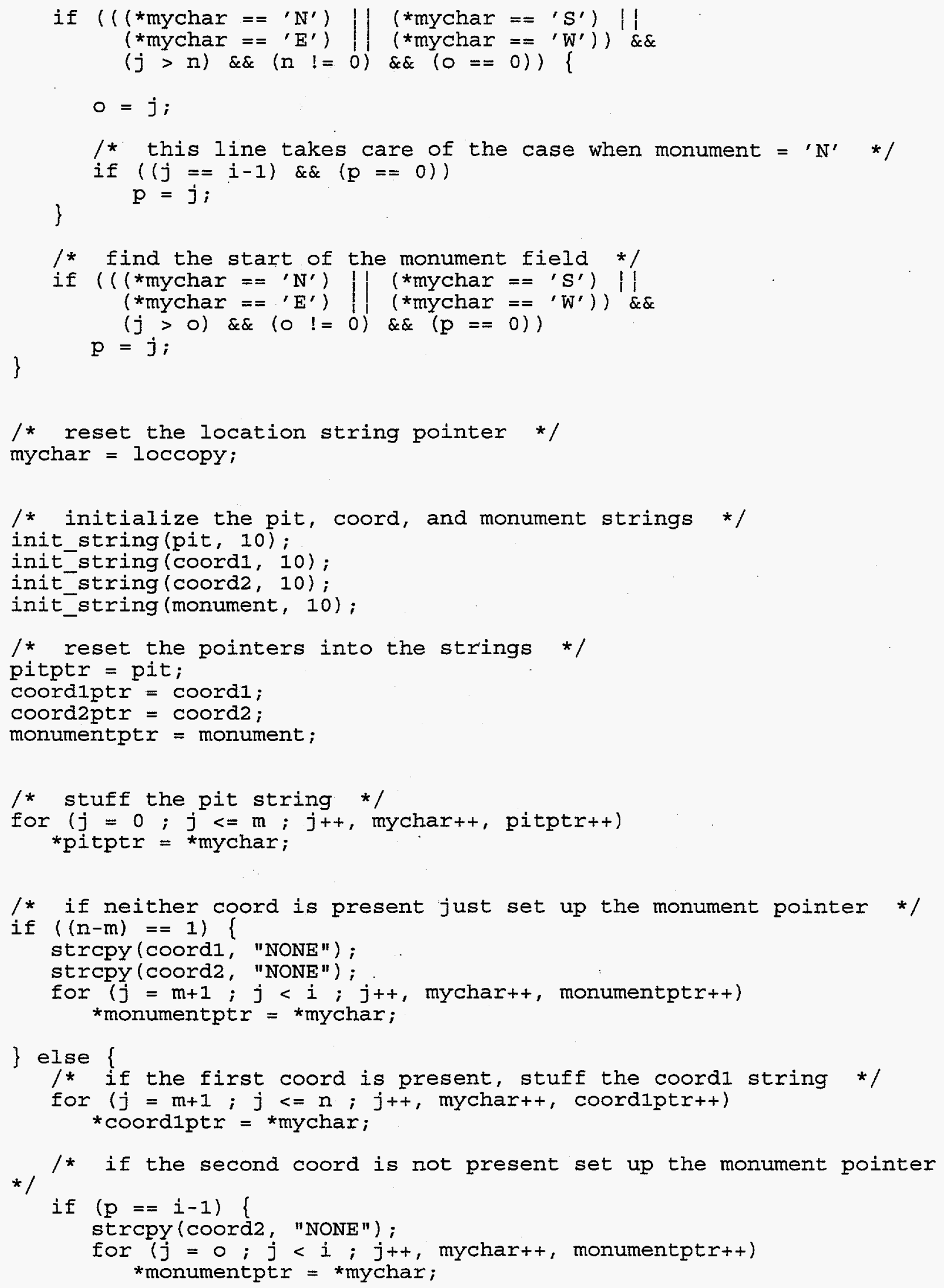

/* if neither coord is present just set up the monument pointer */ 


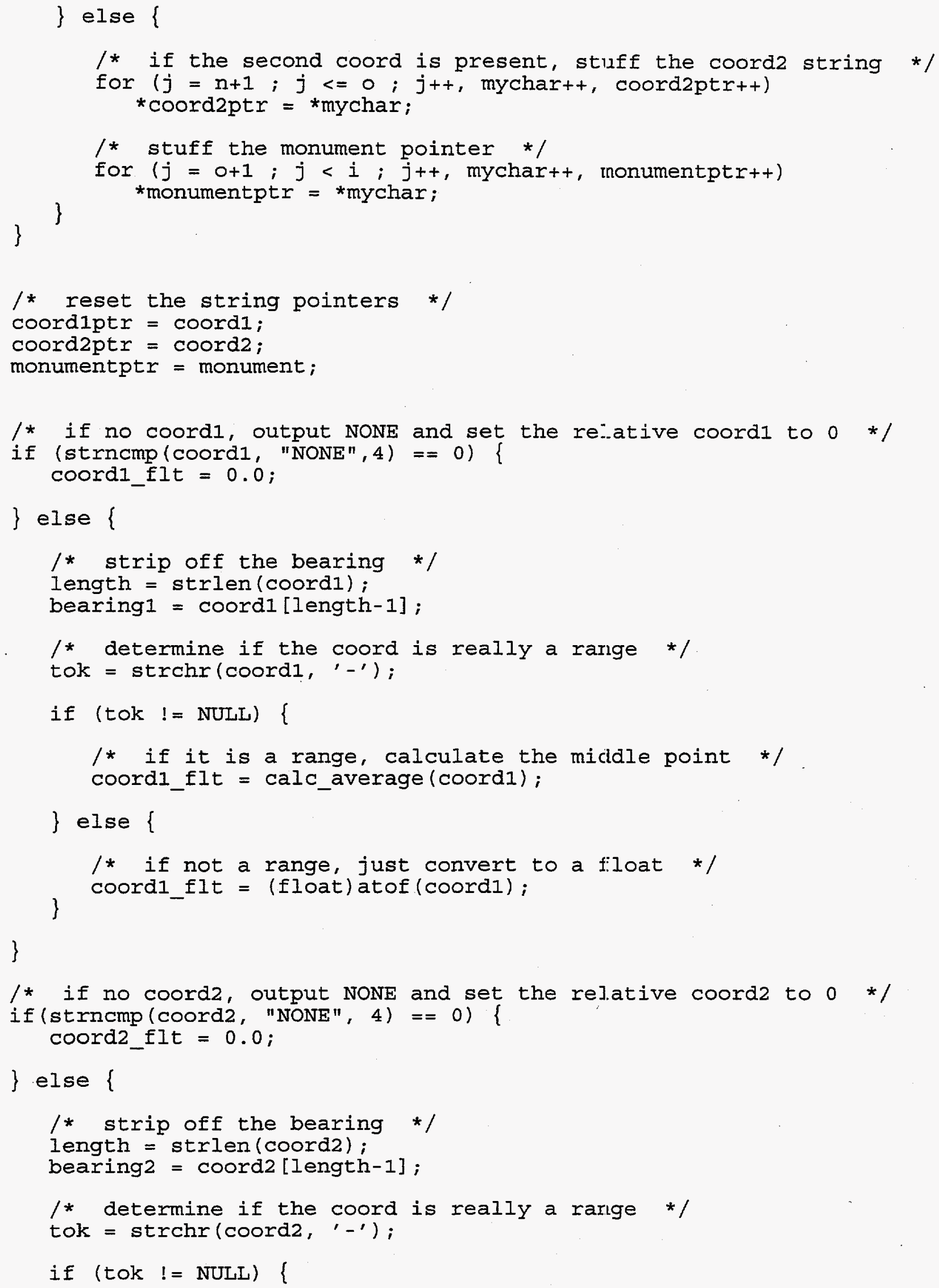

